Iris Winkler / Frederike Schmidt (Hrsg.)

\title{
Interdisziplinäre Forschung in der Deutschdidaktik
}

„Fremde Schwestern“ im Dialog

\section{in/ter/dis/zi/p/li/när [auch 'I...] 〈lat.〉}

(zwischen Disziplinen bestehend; mehrere Disziplinen betreffend); In/ter/dis/zi/p/li/na/rität, die; -

in/te/r/es/sạnt 〈franz. 〉; in/te/r/es-

san/ter/weilse: In/te/r/es/sant- 
Iris Winkler / Frederike Schmidt (Hrsg.)

\section{Interdisziplinäre Forschung in der Deutschdidaktik}

Viele aktuelle Forschungs- und Entwicklungsfragen der Fachdidaktik Deutsch sind nur interdisziplinär - z. B. in Kooperation mit Bildungswissenschaften und anderen Fachdidaktiken - zu bearbeiten. Die Deutschdidaktik forscht deshalb immer öfter in fächerübergreifenden Projekten. Die Beiträge des Sammelbandes gehen der Frage nach, wie sich deutschdidaktische Fragestellungen und Untersuchungsmethoden einerseits und Forschungsparadigmen der kooperierenden Disziplin(en) andererseits produktiv aufeinander beziehen lassen. Vor dem Hintergrund unterschiedlicher Konstellationen und
Ziele der Zusammenarbeit diskutieren die Autorinnen und Autoren Erkenntnisse sowie Herausforderungen und bringen dabei die Perspektiven von Forschenden aus der Deutschdidaktik und aus anderen Fachkulturen miteinander ins Spiel.

\section{Die Herausgeberinnen}

Iris Winkler ist Professorin für Fachdidaktik Deutsch an der Friedrich-SchillerUniversität Jena. Frederike Schmidt ist Wissenschaftliche Mitarbeiterin am Lehrstuhl für Fachdidaktik Deutsch an der Friedrich-SchillerUniversität Jena. 
Interdisziplinäre Forschung in der Deutschdidaktik 


\section{POSITIONEN DER DEUTSCHDIDAKTIK THEORIE UND EMPIRIE \\ Herausgegeben von Christoph Bräuer und Iris Winkler}

\section{BAND 2}


Iris Winkler / Frederike Schmidt (Hrsg.)

\section{Interdisziplinäre Forschung in der Deutschdidaktik}

„Fremde Schwestern“ im Dialog

\section{PETER LANG}




\section{Bibliografische Information der Deutschen Nationalbibliothek}

Die Deutsche Nationalbibliothek verzeichnet diese Publikation in der Deutschen Nationalbibliografie; detaillierte bibliografische Daten sind im Internet über http://dnb.d-nb.de abrufbar.

Gedruckt mit freundlicher Unterstützung der

Friedrich Stiftung, Hannover.

Umschlagabbildung:

(c) Frederike Schmidt

ISSN 2364-1312

ISBN 978-3-631-66340-0 (Print)

E-ISBN 978-3-653-05665-5 (E-PDF)

E-ISBN 978-3-631-69285-1 (EPUB)

E-ISBN 978-3-631-69286-8 (MOBI)

DOI 10.3726/978-3-653-05665-5
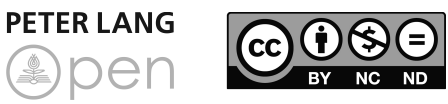

Open Access: Dieses Werk ist lizensiert unter der Creative Commons Lizenz Namensnennung - Nicht kommerziell - Keine Bearbeitungen 4.0 International (CC BY-NC-ND 4.0). Den vollständigen Lizenztext finden Sie unter: https://creativecommons.org/licenses/by-nc-nd/4.0/deed.de

(c) Iris Winkler / Frederike Schmidt, 2016

Peter Lang GmbH

Internationaler Verlag der Wissenschaften

Berlin

Peter Lang - Berlin · Bern · Bruxelles · New York ·

Oxford $\cdot$ Warszawa $\cdot$ Wien

Diese Publikation wurde begutachtet.

www.peterlang.com 


\title{
Inhaltsverzeichnis
}

\author{
Iris Winkler, Frederike Schmidt
}

Interdisziplinäre Forschung in der Deutschdidaktik. Eine Zwischenbilanz.........7

Thomas Boyken

Über wissenschaftliche Verwandtschaftsverhältnisse. Versuch einer

Einordnung der aktuellen Entwicklungen innerhalb der

Deutschdidaktik aus literaturwissenschaftlicher Sicht

Gerhard Rupp

Fremde und vertraute Schwestern - Konstellationen interdisziplinärer

Forschung in der Deutschdidaktik seit den 90er Jahren.

Volker Frederking, Sofie Henschel

Interdisziplinäre Forschung in der Deutschdidaktik am Beispiel des

Projekts Literarästhetische Urteils- und Verstehenskompetenz (LUK)

Katrin Böhme, Petra Stanat, Michael Becker-Mrotzek

Die Entwicklung integrierter Kompetenzstufenmodelle als

Ergebnis interdisziplinärer Kooperation

Ulrike Behrens, Sebastian Weirich

It takes a whole village... Interdisziplinäre Perspektiven bei der

Entwicklung eines Testverfahrens zum Zuhören

Irene Pieper, Florentina Sâmihăian

International Research Cooperation in What Used to Be Called

Mother Tongue Education. The Example of LiFT-2

Iris Winkler, Matthias Heinrich, Astrid Fischer, Ulrike Krause

Multiperspektivität in der Lehrerbildung. Fächerübergreifendes

Lernen in der Mathematik- und Deutschdidaktik 
Frederike Schmidt, Barbara Moschner

„Fremde Schwestern“ und „kritische Freundinnen“ - Lehren und Lernen in einem interdisziplinären Promotionsprogramm in der Lehrerbildung 201

Thomas Lindauer, Claudia Schmellentin, Anne Beerenwinkel Sprachbewusster Naturwissenschafts-Unterricht - Werkstattbericht zu einem transdisziplinären Entwicklungsprojekt 


\title{
Iris Winkler, Frederike Schmidt \\ Interdisziplinäre Forschung in der Deutschdidaktik. Eine Zwischenbilanz
}

\begin{abstract}
Interdisciplinary collaborations have become an established research approach in „Deutschdidaktik“. In this review we discuss the domain specific implications of this fact. Referring to the articles in this volume, we suggest a classification of interdisciplinary research projects that focuses on two main aspects: the synthesis of involved perspectives and the main objective of interest.
\end{abstract}

\section{Ausgangslage}

Der vorliegende Band nimmt die Diskussion auf, die im März 2015 auf der Jenaer Tagung „Fremde Schwestern“ im Dialog. Interdisziplinäre Forschung in der Deutschdidaktik begonnen wurde. ${ }^{1}$ Der Titel der Tagung, der im Untertitel dieses Buches erhalten ist, spielt auf den Beitrag von Ewald Terhart (2002) an. Terhart verweist darin auf das Spannungsverhältnis zwischen Allgemeiner Didaktik und empirischer Lehr-Lern-Forschung und bringt die Beziehung dieser beiden Disziplinen mit dem Bild von den fremden Schwestern auf den Punkt - Schwestern, die sich trotz der Gemeinsamkeiten ihres Forschungsfeldes in ihren wissenschaftstheoretischen Zugriffen auf dieses Feld so grundlegend unterscheiden, dass damit die gegenseitige Verständigung erheblich erschwert ist. Die Fachdidaktik drängt, quasi als dritte „Schwester“, in letzter Zeit verstärkt auf die Bühne, auf der die beiden erstgenannten „Schwestern“ ihr Verhältnis ausfechten; ob in der Rolle der künftigen Integrationsfigur (Terhart 2002, S. 84) oder der zusätzlichen Konkurrentin (Reusser 2008, S. 222), ist noch nicht ausgemacht.

Die Fachdidaktik Deutsch ist von Haus aus ein Hybridfach (vgl. Klein 2010, S. 21f.). Sie ist „inhärent interdisziplinär“ (Lindauer et al., in diesem Band, S. 226) oder, anders gesagt, eine ,transdisziplinäre Disziplin' (Frederking/Henschel, in diesem Band, S. 74); denn sie amalgamiert Elemente zahlreicher Bezugswissenschaften. Man kann anzweifeln, ob die Deutschdidaktik eine eigene Disziplin ist. Sie bildet - auch im Selbstverständnis der meisten ihrer Angehörigen - wohl eher ein eigenes Fach (vgl. Heckhausen 1987, S. 129-131) und eine Unterdisziplin der

1 Wir danken der Friedrich Stiftung für die finanzielle Förderung der Tagung und der vorliegenden Publikation. Kerstin Wuthenow und Ulrike Vorwald gilt unser Dank für die Unterstützung bei der Endredaktion. 
Germanistik, insofern sie sich auf fachbezogene Lehr-Lernprozesse des Schulfachs Deutsch bezieht. Die Frage, wie sie ihr Verhältnis zu ihren Bezugswissenschaften bestimmt - welche Perspektive sie also in ihren Fragestellungen priorisiert -, hat sich für die Deutschdidaktik immer gestellt, und sie ist innerhalb der Deutschdidaktik nie einheitlich beantwortet worden (vgl. z. B. Hurrelmann 1998; Rosebrock 2001; Winkler 2012; Kepser 2013). Die Öffnung für eine Zusammenarbeit mit anderen Fächern resp. Disziplinen erschien aber über lange Zeit kaum geboten, weil Forschende aus der Fachdidaktik Deutsch von ihrer Ausbildung und ihrem Selbstverständnis her Philologie und Pädagogik in Personalunion vertreten ${ }^{2}$, wenn auch mit jeweils individueller Akzentsetzung. Der Gegenstandsbereich der Deutschdidaktik ließ sich aus dieser Warte weitgehend autonom abdecken.

Die Wende zur Empirie und Kompetenzorientierung im Bildungswesen verunsicherte das fachliche Selbstverständnis der Fachdidaktik Deutsch ${ }^{3}$ - und zwar, obwohl sie zu diesem Zeitpunkt schon Erfahrungen in empirischer Forschung vorzuweisen hatte, wie Gerhard Rupp in seinem Überblicksbeitrag aufzeigt. Einschneidend für viele im Fach, insbesondere aus dem wissenschaftlichen Nachwuchs, war in diesem Zusammenhang die Forderung Norbert Groebens beim Symposion Deutschdidaktik 2004 in Lüneburg: Groeben hatte dafür plädiert, dass sich die Fachdidaktik Deutsch empirisch auszurichten habe, wenn sie nicht in der gesellschaftlichen wie wissenschaftlichen Bedeutungslosigkeit versinken wolle (vgl. Groeben 2005, S. 9). Ausgerufen wurde hier nicht weniger als die Notwendigkeit, durch eine Neuausrichtung die eigene fachliche Existenz zu legitimieren. Indem die Diskussion, wie es die Fachdidaktik mit der Empirie zu halten habe, diesem äußeren Rechtfertigungsdruck folgt, zeigt sie zum Teil eine „Tendenz ins Prinzipielle“ (Matuschek 2015, S. 496), die fachliche Überlegungen über eine sinnvolle Ausdifferenzierung der Fachdidaktik in Richtung Empirie zurücktreten lässt. ${ }^{4}$

Aus der partiellen Empirisierung der Deutschdidaktik resultiert fachintern ein Spannungsverhältnis zwischen dem hermeneutisch-geisteswissenschaftlichen

2 Auch aus der Außensicht verschwimmen z. T. Fachwissenschaft und Fachdidaktik. Dies spiegelt sich etwa darin, dass in der Denomination etlicher Professuren Fachdidaktik und Fachwissenschaft kombiniert sind.

3 Die Göttinger Tagung im Frühjahr 2015 spiegelt das aktuelle Ringen um einen verbindenden „Denkrahmen“ der Deutschdidaktik wider und auch, dass diese fachliche Identitätssuche die Deutschdidaktik von ihren Anfängen an begleitet hat (vgl. Bräuer, im Druck).

4 Matuschek (2015) diskutiert ähnliche Probleme von Richtungsdebatten innerhalb der Literaturwissenschaft. Für die Fachdidaktik Deutsch stellt etwa Köster (2015, S. 159) fest, „dass das Verhältnis von Theorie und Empirie [...] eine qualifizierte Debatte braucht“. 
und dem empirisch-szientistischen Forschungsverständnis (vgl. Groeben 2006, S. 287). Folgt man Heckhausen (1987), dann konkurrieren damit innerhalb der Deutschdidaktik zwei „theoretische Integrationsniveau[s]“ (ebd., S. 132). Das theoretische Integrationsniveau ist es, das laut Heckhausen „die Disziplinarität eines Faches ausmacht" (ebd.), weil es den wissenschaftstheoretischen Zugriff auf den ausgewählten Gegenstandsbereich und damit die Art und Weise regelt, wie „die betreffenden Sachverhalte der Wirklichkeit zu verstehen, zu erklären, vorherzusagen, praktisch zu nutzen, zu ändern“ (ebd.) sind. Das Verhältnis des hermeneutischen und empirischen Zugriffs auf deutschdidaktische Forschungsgegenstände konnte innerhalb des Faches nur teilweise produktiv geklärt werden. ${ }^{5}$ Gelingt dies nicht, entfremden sich Angehörige desselben Faches (vgl. dazu Frederking/Henschel, in diesem Band, S. 72).

Der vorliegende Sammelband nimmt Forschungszusammenhänge in den Blick, in denen die Fachdidaktik Deutsch über die Grenzen ihres Faches hinausschaut. Dies geschieht, wenn die eigene fachliche Spezialisierung und Ausdifferenzierung, die sich im Laufe der Zeit herausgebildet hat (vgl. Mittelstraß 1987, S. 153f.), nicht mehr ausreicht, um aktuelle Forschungsfragen zu untersuchen. Die nötige Perspektiverweiterung verlangt Aushandlungsprozesse mit Forschenden aus anderen Wissenschaftsgebieten, d. h. die Verständigung mit mehr oder weniger ,fremden Schwestern. Im Folgenden werden also solche Projekte vorgestellt, in denen die Deutschdidaktik mit anderen Fächern resp. Disziplinen kooperiert, um Forschungs- und Entwicklungsfragen zu lösen, die die Deutschdidaktik in hohem Maß betreffen, aber nicht von ihr allein zu bearbeiten sind. Als Merkmal von Interdisziplinarität reicht dabei die Kooperation nicht aus (Klein 2010, S. 19). Vielmehr geht es im Kern um die Synthese unterschiedlicher fachlicher Perspektiven mit Blick auf eine gemeinsame Problemstellung („Learning to synthesize“, vgl. Boix Mansilla 2010). Die Integration und Interaktion der einzelnen fachlichen Perspektiven kann dabei graduell sehr unterschiedlich ausgeprägt sein (vgl. Klein 2010), wie sich auch in den folgenden Beiträgen zeigt.

\section{Konstellationen und Zielrichtungen}

Bereits Heckhausen (1972, S. 87-89) hat in einem viel zitierten Beitrag verschiedene Abstufungen („stage[s] of maturity“, ebd., S. 87) der interdisziplinären Zusammenarbeit unterschieden. Seine Unterscheidung greifen wir auf, wenn wir die in diesem Band vorgestellten Projekte in ein zweidimensionales Modell interdisziplinärer Forschung einordnen (Abb. 1). Zum einen werden die einzelnen Forschungsprojekte

5 Eine positivere Einschätzung hierzu vertritt Gerhard Rupp, in diesem Band. 
entlang einer Achse zunehmender Interaktion und Integration fachlicher Perspektiven unterschieden. Für diese Achse verwenden wir hier den Begriff Synthesegrad. Unter Synthese verstehen wir das übergreifende Forschungsergebnis, in dem wissenschaftliche Erkenntnisse und methodische Ansätze der beteiligten Fächer bzw. Disziplinen zusammengeführt werden (vgl. Defila/Di Giulio 2015, S. 125f.), so dass im weitreichendsten Fall aus dieser Integration etwas qualitativ Neues entsteht. Die zweite Achse unseres Modells fokussiert das primäre Erkenntnisinteresse interdisziplinärer Projekte, also ob die zu klärende Fragestellung aus gesellschaftlichen Problemen bzw. Anforderungssituationen resultiert oder aus der Wissenschaft selbst stammt. Damit knüpfen wir u. a. an Überlegungen von Mittelstraß (2005) an, der seinerseits Forschungsprojekte nach praktischer und

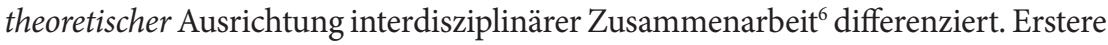
greift außerwissenschaftliche Problemstellungen, also konkrete Notwendigkeiten verschiedener Handlungsfelder, auf; letztere setzt sich mit Problemen auseinander, die in der Wissenschaft selbst aufgeworfen werden. ${ }^{7}$

Abb. 1: Modell interdisziplinärer Forschung

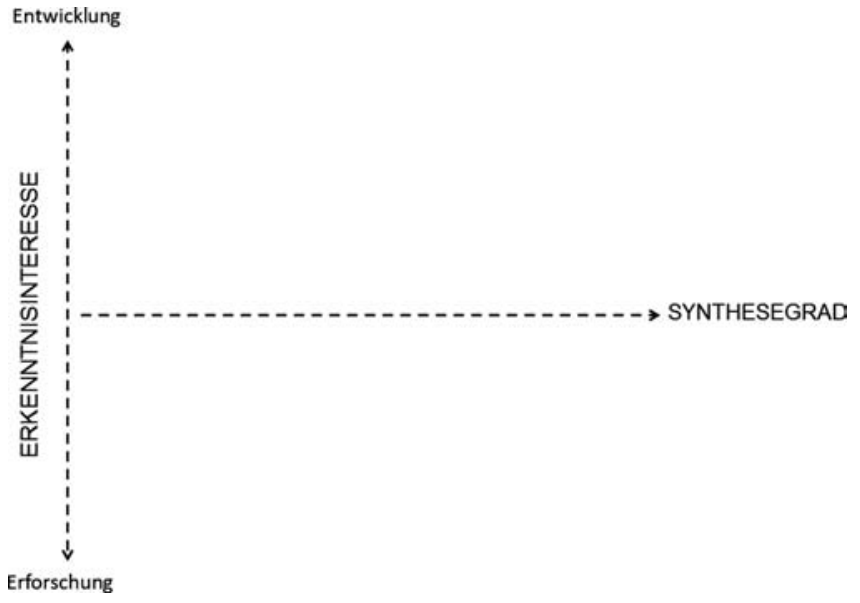

6 Mittelstraß (2005) spricht diesbezüglich von Transdisziplinarität, um Interdisziplinarität auf einem sehr fortgeschrittenen Interaktions- und Integrationsniveau zu bezeichnen (vgl. auch Jungert 2010, S. 6).

7 Vgl. hierzu auch Klein (2010, S. 27), die unter Verweis auf eine OECD-Publikation von 1982 endogene und exogene Interdisziplinarität unterscheidet: „The Endogenous originates within science, while the Exogenous originates in 'real problems of the community' [...]“. Defila/Di Giulio (2015, S. 124) betrachten wissenschaftsgeleitete Projekte als primär „curiosity driven“ und praxisgeleitete Projekte als primär „problem driven“. 
Man kann diskutieren, ob Forschung, an der die Deutschdidaktik beteiligt ist, nicht stets praktische Relevanz für ihren Gegenstandsbereich, den Deutschunterricht, beanspruchen muss (vgl. z. B. Ossner 1993, 1999) und die getroffene Unterscheidung von diesem Hintergrund obsolet ist. Sicher handelt es sich auch hier weniger um eine dichotome als um eine graduelle Differenz. Wir meinen an den Projekten, die in diesem Band vorgestellt werden, zu erkennen, dass der Anstoß für die interdisziplinäre Kooperation mal mehr, mal weniger von Entwicklungsbedarfen der Schulpraxis bzw. der Bildungsadministration getragen oder auf diese ausgerichtet ist (Abb. 2). Eine eigene Variante interdisziplinärer Forschung bilden dabei diejenigen Projekte, in die Mitwirkende aus der außerwissenschaftlichen Praxis einbezogen sind (Behrens/Weirich; Böhme/Stanat/Becker-Mrotzek; Lindauer et al.; Pieper/Sâmihăian). ${ }^{8}$

Da sie von der Anlage der Zusammenarbeit her über die Wissenschaft hinaus gehen, können sie mit Defila/Di Giulio (1999) als transdisziplinär bezeichnet werden - eine Bezeichnung, die Thomas Lindauer, Claudia Schmellentin und Anne Beerenwinkel in ihrem Beitrag aufnehmen. Ob diese Projekte eher ,praktisch' oder ,theoretisch' ausgerichtet sind, hängt allerdings nicht allein von der Einbeziehung von Beteiligten des nicht-akademischen Handlungsfeldes ab.

Abb. 2: Einordnung der Projekte in diesem Band in das Modell interdisziplinärer Forschung

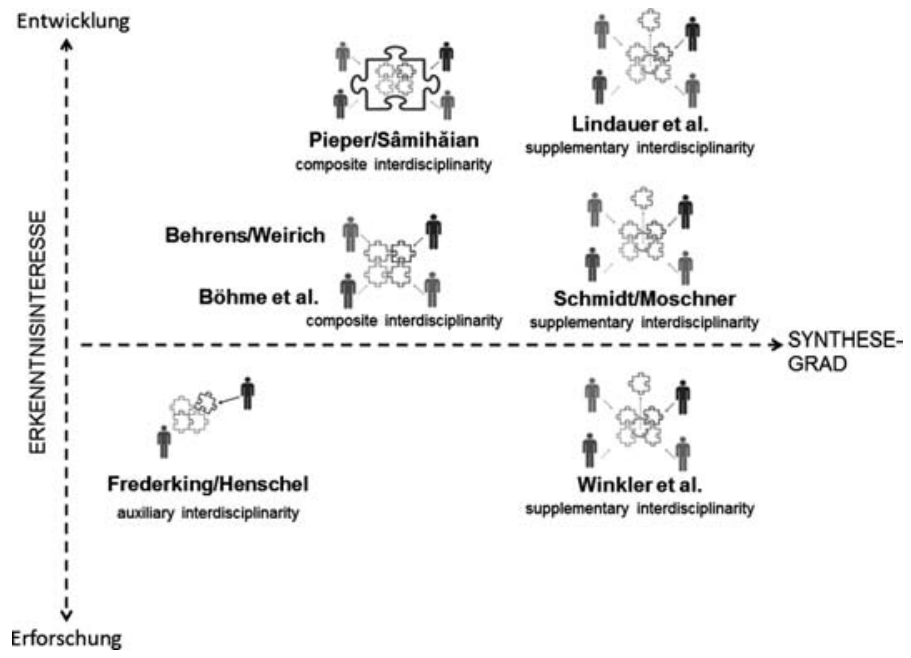

8 Derartige Projekte können wiederum danach unterschieden werden, welchen Stellenwert die Perspektive der Mitwirkenden aus der sog. Praxis hat (vgl. Defila/Di Giulio 2015, S. 128-130). 


\subsection{Erkenntnisinteresse der interdisziplinären Zusammenarbeit: Entwicklungs- oder Forschungsorientierung}

Einige der im skizzierten Sinn ,transdisziplinären' Projekte greifen explizit Entwicklungsbedarfe außerwissenschaftlicher Handlungsfelder auf (vgl. Abb. 2). So zeigen Ulrike Behrens und Sebastian Weirich, wie sich das Projekt stim.mig der virulenten Anforderung stellt, geeignete Aufgabenstellungen für die Überprüfung von Zuhörkompetenz zu konstruieren. Im Rahmen des Projekts Fachlernen und Sprache (Lindauer et al., in diesem Band) werden Materialien und Handreichungen für einen sprachbewussten Fachunterricht entwickelt. Auch das Projekt LIFT folgt einem Entwicklungsziel, das am professionellen Bedarf von Literaturlehrerinnen und -lehrern in Europa ausgerichtet ist und deren Perspektive bereits in der Anlage des Projektes mit einbezieht, wie Irene Pieper und Florentina Sâmihăian in ihrem Beitrag deutlich machen.

Eine Zwischenstellung zwischen Erforschung und Entwicklung nehmen die Projekte ein, die Katrin Böhme, Petra Stanat und Michael Becker-Mrotzek sowie Frederike Schmidt und Barbara Moschner vorstellen. Katrin Böhme et al. geht es im Kontext der KMK-Bildungsstandards darum, empirisch fundierte integrierte Kompetenzstufenmodelle aufzustellen. „Die Entwicklung solcher Kompetenzstufenmodelle erfolgt immer in einem Abstimmungsprozess zwischen Wissenschaft, Schulpraxis und Bildungspolitik" (Böhme et al., in diesem Band, S. 112). Der Auftrag für diese Entwicklung kommt explizit aus der Bildungspolitik. Entsprechende domänenspezifische Kompetenzstufenmodelle sind aber zugleich auch ein Forschungsdesiderat der Fachdidaktik, wenn sie die mit der Kompetenzorientierung verbundenen wissenschaftlichen Herausforderungen ernst nimmt. Das Promotionsprogramm ProfaS, das im Zentrum des Beitrags von Schmidt und Moschner steht, ist als Forschungsprogramm mit empirischer Ausrichtung konzipiert. Von seinem theoretischen Rahmen her zielt es gleichwohl dezidiert auf die Entwicklung begründeter Leitlinien für die Lehrerbildung.

Im Projekt $L U K$, das Volker Frederking und Sofie Henschel in ihrem Beitrag beleuchten, steht eine zunächst vorwiegend wissenschaftsgeleitete Fragestellung im Zentrum, nämlich die theoretische Klärung und empirische Validierung des Konstrukts „Literarästhetische Textverstehenskompetenz“. Auch das Projekt von Iris Winkler, Matthias Heinrich, Astrid Fischer und Ulrike Krause verfolgt in erster Linie das Forschungsziel, die Wirkung von Multiperspektivität in fachdidaktischen Lernumgebungen zu untersuchen. Dass die genannten Klärungen auf längere Sicht für das Anwendungsfeld relevant sein können und sogar sollen, ist von der getroffenen Einordnung nach dem primären Erkenntnisinteresse der interdisziplinären Zusammenarbeit unbenommen. 


\subsection{Synthesegrade interdisziplinärer Forschung}

Die vorgestellten Projekte zeigen, dass in der interdisziplinären Kooperation die beteiligten fachlichen Perspektiven und Wissensbestände graduell unterschiedlich synthetisiert werden. In Anknüpfung an Heckhausen (1972, S. 87-89) sehen wir folgende Synthesegrade ${ }^{9}$ repräsentiert:

Auxiliary Interdisciplinarity: Die kooperierenden Fächer resp. Disziplinen bedienen sich wechselseitig der methodischen Expertise oder des fachlichen Wissens des Partners, um Lücken zu schließen, die aus der eigenen Spezialisierung resultieren. Ausgangs- und Zielgröße der Zusammenarbeit ist die eigene Disziplin. Ein Beispiel hierfür ist das Projekt LUK (Frederking/Henschel, in diesem Band). ${ }^{10}$ Die Einbeziehung von Wissensbeständen und methodischen Zugangsweisen der anderen Disziplin(en) ist also eher auf Kompensation als auf Integration und Synthese ausgerichtet.

Composite Interdisciplinarity: In diesen Projekten, die i. d. R. transdisziplinär im obigen Sinn angelegt sind, ist zum Erreichen des gemeinsamen Ziels die Kombination verschiedener Perspektiven erforderlich, die sich zu einem Ganzen ergänzen (,jigsaw puzzle-like composition“, Heckhausen 1972, S. 88). Die beteiligten Disziplinen teilen streng genommen nicht einmal denselben Gegenstandsbereich noch dasselbe theoretische Integrationsniveau. Einzig die Erfordernisse der spezifischen

9 Dass den dokumentierten Projekten weiterhin differente Theorien von Forschungsund Entwicklungsarbeit zugrunde liegen, kann hier nicht vertiefend diskutiert werden. Zur Rolle von Theorien und Theorienbildung als expliziter oder impliziter Rahmenbedingung von Forschungs- und Entwicklungspraktiken vgl. Prediger (2015).

10 Ein solcher kompensatorischer Ansatz interdisziplinärer Zusammenarbeit leitet etwa die Beteiligten aus der Psychologie im DFG-Schwerpunktprogramm Kompetenzmodelle zur Erfassung individueller Lernergebnisse und zur Bilanzierung von Bildungsprozessen, in dem auch das Projekt $L U K$ mit deutschdidaktischer Beteiligung angesiedelt ist (vgl. Frederking/Henschel, in diesem Band). Dazu führen Klieme/Leutner (2006, S. 880f.) aus: „Der Anspruch, kontextspezifische, erlernbare und vermittelbare Leistungsdispositionen zu erfassen, die für die Bewältigung von Anforderungen in konkreten Domänen erforderlich sind, stellt eine besondere Herausforderung dar. Die psychologische Grundlagenforschung und die Psychometrie sind diesem Anspruch bislang noch keineswegs gerecht geworden. Sie müssen ihre Modelle domänenspezifisch kontextualisieren und sind dabei auf eine Zusammenarbeit sowohl mit Erziehungswissenschaftlern als auch mit Domänenexperten, etwa aus den Fachdidaktiken, angewiesen. Diese interdisziplinäre Kooperation soll das Schwerpunktprogramm leisten." Interessant ist vor diesem Hintergrund, dass Sofie Henschel in Bezug auf das Projekt LUK betont, es sei in derartigen Projekten wichtig, „der eigenen Disziplin und deren Qualitätsstandards treu zu bleiben" (Frederking/Henschel, in diesem Band, S. 90). 
Problemstellung verbinden die Partner. Diese Konstellation sehen wir im Projekt stim.mig (Behrens/Weirich), im Projekt LIFT-2 ${ }^{11}$ und bei der Entwicklung integrierter Kompetenzstufenmodelle am IQB (Böhme et al.) gegeben. Heckhausen (1987, S. 138) betont zwar, dass die kombinierten Perspektiven und Wissensbestände bei dieser Variante der Interdisziplinarität nebeneinander stehen bleiben - in diesem Sinn käme es nicht zur weiterführenden Synthese. Unseres Erachtens sind aber Wechselwirkungen zwischen den Perspektiven im Prozessverlauf als Begleiteffekt durchaus erwartbar. Entsprechend heben z. B. Behrens und Weirich in ihrem Beitrag hervor, dass ein

wesentliches Charakteristikum solcher interdisziplinären Projekte ist, dass die Perspektiven nicht mehr einzelnen Personen(-gruppen) strikt zugeordnet werden können. Vielmehr ergibt sich im gemeinsamen Arbeitsprozess erkennbar eine gegenseitige Qualifikation aller Beteiligten. (Behrens/Weirich, in diesem Band, S. 150)

Damit ist bereits ein fließender Übergang zur nächsten Synthesestufe interdisziplinärer Zusammenarbeit markiert.

Supplementary Interdisciplinarity: Diese Form von Interdisziplinarität findet i. d. R. im Grenzbereich der beteiligten Disziplinen statt und basiert auf einer partiellen Überschneidung der disziplinären Gegenstandsbereiche. Zwischen den kooperierenden Disziplinen können kategoriale Unterschiede bestehen, z. B. im

11 Beim Projekt LIFT-2 kann man sich fragen, inwieweit es überhaupt als interdisziplinär zu bezeichnen ist und ob hier nicht schlicht, international die zutreffendere Einordnung wäre. Aus unserer Sicht verdeutlicht der Beitrag von Pieper/Sâmihăian, dass L1-Didaktiken in den verschiedenen europäischen Ländern zwar einen gemeinsamen Bezugspunkt im Handlungsfeld haben, nämlich den Sprach- und Literaturunterricht in der jeweiligen Landessprache. Allerdings führen die landesspezifischen, von unterschiedlichen (bildungs-)kulturellen normativen Setzungen getragenen Ausformungen sowohl des Unterrichts in der Landessprache als auch der zugehörigen L1-Didaktiken zu bedeutsamen Unterschieden: Im gemeinsamen Forschungsprojekt sind weder der Untersuchungsbereich der beteiligten L1-Didaktiken (der jeweilige landessprachliche Unterricht) noch deren disziplinäres Selbstverständnis und ihr Blickwinkel auf das Untersuchungsfeld deckungsgleich (zu den bestimmenden Merkmalen von Disziplinarität vgl. z. B. Heckhausen 1972, S. 83-86). Es müssen also nicht nur (wie in jedem internationalen Forschungsprojekt) interkulturelle Unterschiede gehandhabt werden, sondern auch mehr oder weniger abweichende disziplinäre Ausrichtungen. Wie es kennzeichnend für Composite Interdisciplinarity ist, stehen die landesspezifischen Zugänge im Ergebnis in einer Ergänzungsrelation nebeneinander. Der gemeinsame Referenzrahmen für die Entwicklung literarischer Kompetenzen jedoch rahmt im konkreten Wortsinn die Forschungsergebnisse und gibt ihnen trotz der sehr unterschiedlichen Kontexte eine gemeinsame Basis. 
theoretischen Integrationsniveau. Die gemeinsame Zielstellung sorgt jedoch dafür, dass im Rahmen des Projekts systematisch Gemeinsamkeiten in den Blick genommen werden: „The correspondence is looked for and tentatively established in order to reconstruct life or social processes more fully" (Heckhausen 1972, S. 88). Exemplarisch für diese Herangehensweise stehen die Projekte von Schmidt/Moschner, Lindauer et al. sowie Winkler et al. im vorliegenden Band. Ihre Untersuchungsgegenstände sind in einer Schnittmenge der Forschungsfelder der beteiligten Disziplinen bzw. Fächer platziert. Vor diesem Hintergrund wird von Unterschieden zwischen den Kooperationspartnern ${ }^{12}$ auf der einen Seite abstrahiert und die Gemeinsamkeit gesucht; auf der anderen Seite werden diese Unterschiede im Sinne des Untersuchungsvorhabens aber auch gezielt produktiv gemacht. Intention und Ergebnis des Forschungsprozesses ist die synthetisierende Weiterentwicklung der fachbezogenen Kenntnisse und Sichtweisen. Diese Synthese kann an einem materiellen Produkt sichtbar werden wie beispielsweise anhand von Handreichungen und Materialien für sprachbewussten Unterricht (vgl. Lindauer et al., in diesem Band). Sie kann darüber hinaus in ein verändertes Verständnis der Projektbeteiligten von ihrer eigenen Disziplin münden (vgl. dazu z. B. den Beitrag von Schmidt/Moschner).

\section{Erträge und Rückwirkungen}

Wir haben den Literaturwissenschaftler Thomas Boyken gebeten, die Beiträge dieses Bandes aus der Außensicht zu kommentieren. Boyken plädiert für eine „enge Verzahnung von Fachwissenschaft und Fachdidaktik“ (S. 39), die er in den hier dokumentierten Forschungsprojekten für unterrepräsentiert hält. ${ }^{13}$ Grundlegend ist seiner Forderung aus fachdidaktischer Perspektive zuzustimmen. „Wenn der Faden zur Fachwissenschaft reißt, so verkommt die Fachdidaktik

12 Essentielle Unterschiede bestehen in verschiedenen Fachdidaktiken etwa im jeweils dominierenden Modus der Weltbegegnung (vgl. Baumert 2002, S. 106-108) in den Unterrichtsfächern, auf die sich die Fachdidaktiken jeweils beziehen. Reusser (2008, S. 222) verweist zudem auf das Problem einer ,immer noch fehlenden gemeinsamen Fachsprache unter den verschiedenen Fachdidaktiken“ (Herv. ebd.). Mit Bildungswissenschaften und (Fach-)Didaktiken begegnen sich zudem unterschiedliche theoretische Integrationsniveaus (empirisch-quantitativ ausgerichtet vs. hermeneutisch-qualitativ).

13 Dass auf der Jenaer Tagung kein Fachwissenschaftler aus der Germanistik zu Wort kam, war allein der kurzfristigen Absage des Referenten aus der Literaturwissenschaft geschuldet und hatte keinerlei konzeptionelle Gründe. 
zur Technologie", so bereits Ossner (1999, S. 33). ${ }^{14}$ Auch wenn man Boyken nicht gänzlich zustimmen möchte, dass „die empirische Wende tatsächlich zu einer Trennung von Gegenstandsorientierung und methodischer Vermittlung [verführt]" (Boyken, in diesem Band, S. 38), so wird anhand seiner Kritik dennoch deutlich, dass sich die Fachdidaktik neu auf den Stellenwert der fachlichen Gegenstände im Unterricht besinnen muss. Auf der Tagung zu Interpretationskulturen in Fachdidaktik und Fachwissenschaft, die im Frühjahr 2014 an der HU Berlin stattgefunden hat (Lessing-Sattari et al. 2015), zeigte sich in diesem Zusammenhang zum einen, dass die Literaturdidaktik den Dialog mit der Literaturwissenschaft nicht scheut. Zum anderen aber identifiziert Juliane Köster mit Blick auf die literaturdidaktischen Beiträge der Tagung folgende Problembereiche und unterstreicht damit Boykens Diagnose:

Nichtgeltung des Gegenstands, Defizit im Bereich der Theorieorientierung, Suspendierung der Erlebnisqualität literarischer Texte durch Methodisierung, Suspendierung der Besonderheit des Einzeltextes durch Orientierung am Kompetenzerwerb. (Köster 2015, S. 22)

Gegenwärtig finden sich trotz geteilter Kernfragen keine Forschungsprojekte, in denen Fachwissenschaft und Fachdidaktik kooperieren; die Diskurse berühren sich kaum, was nicht nur an der Fachdidaktik liegen muss. Etwas polemisch gefragt: Wie weit sind die Familienverhältnisse hier mitunter vom „Nicht-zurKenntnis-Nehmen“ entfernt, das Terhart (2002, S. 77) als prägend für das Verhältnis von Allgemeiner Didaktik und empirischer Lehr-Lernforschung sieht? ${ }^{15}$

Dass die hier beschriebene Entwicklung und damit verbundene Fragen nicht nur in der Deutschdidaktik virulent sind, zeigt ein Blick auf die Naturwissenschaftsdidaktiken (vgl. Maurer 2016). Parallelen zu bisherigen Ausführungen zur Situation der Deutschdidaktik werden deutlich, wenn hier kritisch gefragt wird,

ob das Pendel der Forschungsaktivitäten zu weit in Richtung einer empirischen Bildungsforschung geschwungen ist, in der die Spezifika der fachdidaktischen Perspektive nicht mehr genügend deutlich werden. (Schecker/Parchmann/Starauscheck 2016, S. 25)

Sollte die (anscheinend übergreifend zu erkennende) Entfremdung zwischen Fachdidaktik und Fachwissenschaft tatsächlich ein Ergebnis der Kompetenzorientierung und der empirischen Wende sein, so lässt sich diese Entwicklung

$14 \mathrm{Zu}$ welchen Konsequenzen es führen kann, wenn eine zu starke Distanz zwischen Fachdidaktik und Fachwissenschaft entsteht, verdeutlicht etwa die Kontroverse um den Karlsruher Physikkurs (KPK) zwischen Fachphysik und Physikdidaktik (vgl. u. a. Rincke 2014).

15 Auf die Problematik des fehlenden Diskurses für die Lehrerbildung kann man hier nicht näher eingehen. 
aus fachdidaktischer Sicht allerdings auch ein Stück weit positiv wenden. Durch sie scheint die Fachdidaktik in den letzten Jahren eigenständig geworden zu sein (in der Metapher: die ,kleine' Schwester ist ,erwachsen' geworden). Dieser Autonomiegewinn ist eine wichtige Voraussetzung dafür, dass Fachdidaktik und Fachwissenschaft in einen Dialog ,auf Augenhöhe' treten können, wie es für interdisziplinäres Arbeiten auf Forschungsebene wichtig ist. ${ }^{16}$

Die Kooperation mit den vermeintlich ,fremden Schwestern' aus der Wissenschaft, allen voran der Psychologie, scheint mit Blick auf die in diesem Band vorgestellten Projekte eingespielt zu sein. Das alte Problem der Fachdidaktik, im „Hase-und-Igel-Spiel“ mit der empirischen Lehr- und Lernforschung immer zu spät zu kommen (vgl. Bremerich-Vos 1999, S. 72), besteht so offenbar nicht mehr. ${ }^{17}$ Die Fachdidaktik hat aufgeholt; dass sie sich dadurch verändert hat sicher nicht immer von der Logik des eigenen Faches getrieben -, ist bereits angeklungen (s. o., Abschnitt 1).

Eine Herausforderung, die insbesondere mit den transdisziplinär angelegten Forschungsprojekten (s. o., Abschnitt 2) verbunden ist, aber die Implementation fachdidaktischer Forschungsbefunde ins Handlungsfeld insgesamt betrifft, liegt in der Verständigung mit den Akteuren in Deutschunterricht und Bildungsadministration. Wenn diese Verständigung nicht gelingt, führt das "to a practice that disregards research achievements and to research that neglects practical needs" (Defila/ Di Giulio 2015, S. 124). Dies ist kein neues Problem der Fachdidaktik Deutsch, aber vor dem Hintergrund interdisziplinärer Forschung in der Deutschdidaktik aus neuer Perspektive relevant (vgl. dazu auch Rupp, in diesem Band, S. 63f.).

Insgesamt spiegeln die in diesem Band dokumentierten Projekte das gewonnene Selbstbewusstsein der Deutschdidaktik als wissenschaftlicher (Teil-)Disziplin. Sie wirken zugleich zurück auf das Selbstverständnis Deutschdidaktik. Interdisziplinarität kann also ein Motor für disziplinäre Entwicklung sein. Man ist nach der Kooperation meist nicht mehr dieselbe, die man vorher war, weil man sich selbst

16 Zumindest in der Lehre besteht dieser Dialog an einzelnen Universitäten (vgl. z. B. Freudenberg et al. 2014). Die aufgeführten Beobachtungen machen weiterhin Antworten auf die Frage notwendig, welche Projekte bzw. Forschungsschwerpunkte für die Kooperation zwischen Fachwissenschaft und Fachdidaktik gewinnbringend sein können (vgl. für mögliche Antworten die Ausführungen von Boyken, in diesem Band, sowie Wieser 2015, S. 56f.).

17 Zumindest mit Blick auf drittmittelgeförderte Forschungsprojekte muss allerdings kritisch gefragt werden, inwiefern die Fachdidaktik in der Zusammenarbeit nicht manchmal auch „rein als Zulieferer von Inhalten für Projekte wahrgenommen“ wird (Schecker/Parchmann/Starauscheck 2016, S. 27; vgl. auch oben, Fußnote 10). 
mit fremden Augen betrachtet hat. Dabei erkennt man Perspektivverengungen des eigenen Faches, aber eben auch nicht nur diese. Mittelstraß (1987, S. 157) hat darauf hingewiesen, „daß Interdisziplinarität im eigenen Kopf anfangen muß - als Querdenken, Fragen, wohin noch niemand gefragt hat, Lernen, was die eigene Disziplin nicht weiß“. Diese Denkhaltung ist nicht nur Voraussetzung für interdisziplinäre Forschung, wie sie die Beiträge in diesem Band vorstellen, sondern zugleich deren gemeinsamer Ertrag für die Beteiligten.

\section{Literatur}

Baumert, Jürgen (2002): Deutschland im internationalen Bildungsvergleich. In: Killius, Nelson/Kluge, Jürgen/Reisch, Linda (Hrsg.): Die Zukunft der Bildung. Frankfurt a. M.: Suhrkamp, S. 100-150.

Behrens, Ulrike/Weirich, Sebastian (2016): It takes a whole village... Interdisziplinäre Perspektiven bei der Entwicklung eines Testverfahrens zum Zuhören. In: Winkler, Iris/Schmidt, Frederike (Hrsg.): Interdisziplinäre Forschung in der Deutschdidaktik. „Fremde Schwestern“ im Dialog. Frankfurt a. M.: Lang, S. 133-152.

Böhme, Katrin/Stanat, Petra/Becker-Mrotzek, Michael (2016): Die Entwicklung integrierter Kompetenzstufenmodelle als Ergebnis interdisziplinärer Kooperation. In: Winkler, Iris/Schmidt, Frederike (Hrsg.): Interdisziplinäre Forschung in der Deutschdidaktik. „Fremde Schwestern“ im Dialog. Frankfurt a. M.: Lang, S. 101-131.

Boix Mansilla, Veronica (2010): Learning to synthesize: the development of interdisciplinary understanding. In: Frodeman, Robert/Klein, Julia Thompson/ Mitcham, Carl/Holbrook, J. Britt (Eds.): The Oxford Handbook of Interdisciplinarity. Oxford: University Press, pp. 288-306.

Boyken, Thomas (2016): Über wissenschaftliche Verwandtschaftsverhältnisse. Versuch einer Einordnung der aktuellen Entwicklungen innerhalb der Deutschdidaktik aus literaturwissenschaftlicher Sicht. In: Winkler, Iris/Schmidt, Frederike (Hrsg.): Interdisziplinäre Forschung in der Deutschdidaktik. „Fremde Schwestern“ im Dialog. Frankfurt a. M.: Lang, S. 23-41.

Bräuer, Christoph (Hrsg.) (im Druck): Denkrahmen der Deutschdidaktik. Die Identität der Disziplin in der Diskussion. Frankfurt a. M.: Lang.

Bremerich-Vos, Albert (1999): „Qualitätsentwicklung“ und „Qualitätssicherung“ in NRW - und die Deutschdidaktik? In: Didaktik Deutsch. H. 7, S. 65-78.

Defila, Rico/Di Giulio, Antonietta (1999): Evaluationskriterien für inter- und transdisziplinäre Forschung. In: Panorama. Sondernummer 99, S. 5-11. 
Defila, Rico/Di Giulio, Antonietta (2015): Integrating knowledge. Challenges raised by the "Inventory of Synthesis". In: Futures. No. 65, pp. 123-135.

Frederking, Volker/Henschel, Sofie (2016): Interdisziplinäre Forschung in der Deutschdidaktik am Beispiel des Projekts, Literarästhetische Urteils- und Verstehenskompetenz' (LUK). In: Winkler, Iris/Schmidt, Frederike (Hrsg.): Interdisziplinäre Forschung in der Deutschdidaktik. „Fremde Schwestern“ im Dialog. Frankfurt a. M.: Lang, S. 71-99.

Freudenberg, Ricarda/Winkler, Iris/Gallmann, Peter/von Petersdorff, Dirk (2014): Von der Fachwissenschaft über die Fachdidaktik in den Schulunterricht und zurück - Ein Veranstaltungskonzept. In: Kleinespel, Karin (Hrsg.): Ein Praxissemester in der Lehrerbildung. Konzepte, Befunde und Entwicklungsperspektiven am Beispiel des Jenaer Modells der Lehrerbildung. Bad Heilbrunn: Klinkhardt, S. 162-176.

Groeben, Norbert (2005): Auf dem Weg zu einer deutsch-didaktischen Unterrichtsforschung? In: Stückrath, Jörn/Strobel, Ricarda (Hrsg.): Deutschunterricht empirisch. Beiträge zur Überprüfbarkeit von Lernfortschritten im Sprach-, Literatur- und Medienunterricht. Baltmannsweiler: Schneider Hohengehren, S. 7-33.

Groeben, Norbert (2006): Wissenschaftstheorie: Grundlagen für eine Verbindung von empirischen und hermeneutischen Traditionen. In: Groeben, Norbert/ Hurrelmann, Bettina (Hrsg.): Empirische Unterrichtsforschung in der Literatur- und Lesedidaktik. Ein Weiterbildungsprogramm. Weinheim u. München: Juventa, S. 287-306.

Heckhausen, Heinz (1972): Discipline and interdisciplinarity. In: OECD (Ed.): Interdisciplinarity. Problems of teaching and research in universities. Paris: OECD, Centre for Educational Research and Innovation, pp. 83-89.

Heckhausen, Heinz (1987): „Interdisziplinäre Forschung“ zwischen Intra-, Multiund Chimären-Disziplinarität. In: Kocka, Jürgen (Hrsg.): Interdisziplinarität. Praxis - Herausforderung - Ideologie. Frankfurt a. M.: Suhrkamp, S. 129-145.

Hurrelmann, Bettina (1998): Deutschdidaktik - kein Ort, nirgends? In: Didaktik Deutsch. Sonderheft 1998, S. 13-38.

Jungert, Michael (2010): Was zwischen wem und warum eigentlich? Grundsätzliche Fragen der Interdisziplinarität. In: Jungert, Michael/Romfeld, Elsa/Sukopp, Thomas/Voigt, Uwe (Hrsg.): Interdisziplinarität. Theorie, Praxis, Probleme. Darmstadt: Wissenschaftliche Buchgesellschaft, S. 3-12.

Kepser, Mathis (2013): Deutschdidaktik als eingreifende Kulturwissenschaft. Ein Positionierungsversuch im wissenschaftlichen Feld. In: Didaktik Deutsch. H. 34, S. 52-68. 
Klein, Julie Thompson (2010): A taxonomy of interdisciplinarity. In: Frodeman, Robert/Klein, Julia Thompson/Mitcham, Carl/Holbrook, J. Britt (Eds.): The Oxford Handbook of Interdisciplinarity. Oxford: Oxford University Press, pp. 15-30.

Klieme, Eckhard/Leutner, Detlev (2006): Kompetenzmodelle zur Erfassung individueller Lernergebnisse und zur Bilanzierung von Bildungsprozessen. Beschreibung eines neu eingerichteten Schwerpunktprogramms der DFG. In: Zeitschrift für Pädagogik. Jg. 52. H. 6, S. 876-903.

Köster, Juliane (2015): Die Tagung aus literaturdidaktischer Beobachterperspektive. In: Lessing-Sattari, Marie/Löhden, Maike/Meissner, Almuth/Wieser, Dorothee (Hrsg.): Interpretationskulturen. Literaturdidaktik und Literaturwissenschaft im Dialog über Theorie und Praxis des Interpretierens. Frankfurt a. M. u. a.: Lang, S. 11-35.

Lessing-Sattari, Marie/Löhden, Maike/Meissner, Almuth/Wieser, Dorothee (Hrsg.) (2015): Interpretationskulturen. Literaturdidaktik und Literaturwissenschaft im Dialog über Theorie und Praxis des Interpretierens. Frankfurt a. M. u. a.: Lang.

Lindauer, Thomas/Schmellentin, Claudia/Beerenwinkel, Anne (2016): Sprachbewusster Naturwissenschafts-Unterricht - Werkstattbericht zu einem transdisziplinären Entwicklungsprojekt. In: Winkler, Iris/Schmidt, Frederike (Hrsg.): Interdisziplinäre Forschung in der Deutschdidaktik. „Fremde Schwestern“ im Dialog. Frankfurt a. M.: Lang, S. 225-246.

Matuschek, Stefan (2015): Welche Krise? Allgemeine, spezifische und Verständigungs-Probleme der Literaturwissenschaft. In: Deutsche Vierteljahresschrift für Literaturwissenschaft und Geistesgeschichte. Jg. 89. H. 4, S. 494-504.

Maurer, Christian (Hrsg.) (2016): Authentizität und Lernen - das Fach in der Fachdidaktik. Gesellschaft für Didaktik der Chemie und Physik, Jahrestagung in Berlin 2015. Regensburg: Universität Regensburg. http://gdcp.de/images/ tagungsbaende/GDCP_Band36.pdf. Abgerufen am 15.04.2016.

Mittelstraß, Jürgen (1987): Die Stunde der Interdisziplinarität? In: Kocka, Jürgen (Hrsg.): Interdisziplinarität. Praxis - Herausforderung - Ideologie. Frankfurt a. M.: Suhrkamp, S. 152-157.

Mittelstraß, Jürgen (2005): Methodische Transdisziplinarität. In: Technikfolgenabschätzung. Theorie und Praxis. Jg. 14. H. 2, S. 18-23.

Ossner, Jakob (1993): Praktische Wissenschaft. In: Bremerich-Vos, Albert (Hrsg.): Handlungsfeld Deutschunterricht im Kontext. Festschrift für Hubert Ivo zum 65. Geburtstag. Frankfurt a. M.: Diesterweg, S. 186-199.

Ossner, Jakob (1999): Das Profil der Fachdidaktik. Grundzüge einer praktischen Disziplin. In: Radtke, Frank-Olaf (Hrsg.): Lehrerbildung an der Universität. 
Zur Wissensbasis pädagogischer Professionalität. Frankfurter Beiträge zur Erziehungswissenschaft. Frankfurt a. M.: Johann Wolfgang Goethe-Universität, S. 24-45.

Pieper, Irene/Sâmihăian, Florentina (2016): International Research Cooperation in What Used to Be Called Mother Tongue Education. The Example of LiFT2. In: Winkler, Iris/Schmidt, Frederike (Hrsg.): Interdisziplinäre Forschung in der Deutschdidaktik. „Fremde Schwestern“ im Dialog. Frankfurt a. M.: Lang, S. $153-180$.

Prediger, Susanne (2015): Theorien und Theoriebildung in didaktischer Forschung und Entwicklung. In: Bruder, Regina/Hefendehl-Hebeker, Lisa/SchmidtThieme, Barbara/Weigand, Hans-Georg (Hrsg.): Handbuch der Mathematikdidaktik. Berlin/Heidelberg: Springer, S. 643-662.

Reusser, Kurt (2008): Empirisch fundierte Didaktik - didaktisch fundierte Unterrichtsforschung. Eine Perspektive zur Neuorientierung der Allgemeinen Didaktik. In: Zeitschrift für Erziehungswissenschaft. Jg. 10. Sonderheft, S. 219-237.

Rincke, Karsten (2014): Die Deutsche Physikalische Gesellschaft und ihr Verhältnis zur Didaktik der Physik. Fünf Thesen und ein Aufruf zum Dialog. In: Regensburger Dialoge. H. 1. Regensburg: Didaktik der Physik. http://www. physik.uni-regensburg.de/forschung/rincke/Allgemeines/thesen_rincke14. pdf. Abgerufen am 17.04.2016.

Rosebrock, Cornelia (2001): Einleitung. In: Rosebrock, Cornelia/Fix, Martin (Hrsg.): Tumulte. Deutschdidaktik zwischen den Stühlen. Baltmannsweiler: Schneider Hohengehren, S. 2-5.

Rupp, Gerhard (2016): Fremde und vertraute Schwestern - Konstellationen interdisziplinärer Forschung in der Deutschdidaktik seit den 90er Jahren. In: Winkler, Iris/Schmidt, Frederike (Hrsg.): Interdisziplinäre Forschung in der Deutschdidaktik. „Fremde Schwestern“ im Dialog. Frankfurt a. M.: Lang, S. 43-69.

Schecker, Horst/Parchmann, Ilka/Starauscheck, Erich (2016): Fachlichkeit der Fachdidaktik - Standortbestimmung und Perspektiven (Workshop). In: Maurer, Christian (Hrsg.): Authentizität und Lernen - das Fach in der Fachdidaktik. Gesellschaft für Didaktik der Chemie und Physik, Jahrestagung in Berlin 2015. Regensburg: Universität Regensburg, S. 25-28. http://gdcp.de/images/ tb2016/TB2016_25_Schecker.pdf. Abgerufen am 15.04.2016.

Schmidt, Frederike/Moschner, Barbara (2016): „Fremde Schwestern“ und „kritische Freundinnen" - Lehren und Lernen in einem interdisziplinären Promotionsprogramm in der Lehrerbildung. In: Winkler, Iris/Schmidt, Frederike (Hrsg.): Interdisziplinäre Forschung in der Deutschdidaktik. „Fremde Schwestern“ im Dialog. Frankfurt a. M.: Lang, S. 201-223. 
Terhart, Ewald (2002): Fremde Schwestern. Zum Verhältnis von Allgemeiner Didaktik und empirischer Lehr-Lern-Forschung. In: Zeitschrift für Pädagogische Psychologie. Jg. 16. H. 2, S. 77-86.

Wieser, Dorothee (2015): Interpretationskulturen: Überlegungen zum Verhältnis von theoretischen und praktischen Problemen in Literaturwissenschaft und Literaturunterricht. In: Lessing-Sattari, Marie/Löhden, Maike/Meissner, Almuth/Wieser, Dorothee (Hrsg.): Interpretationskulturen. Literaturdidaktik und Literaturwissenschaft im Dialog über Theorie und Praxis des Interpretierens. Frankfurt a. M. u. a.: Lang, S. 39-60.

Winkler, Iris (2012): Wozu Literaturdidaktik? Perspektiven auf eine Disziplin zwischen den Stühlen. In: Doering, Sabine/Wätjen, Hans-Joachim (Hrsg.): Oldenburger Universitätsreden. Nr. 200. Oldenburg: BIS-Verlag, S. 9-31.

Winkler, Iris/Heinrich, Matthias/Fischer, Astrid/Krause, Ulrike (2016). Multiperspektivität in der Lehrerbildung. Fächerübergreifendes Lernen in der Mathematik- und Deutschdidaktik. In: Winkler, Iris/Schmidt, Frederike (Hrsg.): Interdisziplinäre Forschung in der Deutschdidaktik. „Fremde Schwestern“ im Dialog. Frankfurt a. M.: Lang, S. 181-199. 


\title{
Thomas Boyken
}

\section{Über wissenschaftliche Verwandtschaftsverhältnisse. Versuch einer Einordnung der aktuellen Entwicklungen innerhalb der Deutschdidaktik aus literaturwissenschaftlicher Sicht}

\begin{abstract}
From an external perspective, the paper gives a comment on recent research developments und processes in Didactics of German Literature and Language. The paper claims at last the strong correlation between Didactics of German Literature and Language and German literature studies, not only in the education of German school teachers.
\end{abstract}

\section{Die Metapher der,fremden Schwestern}

Um das Verhältnis von Deutschdidaktik und verschiedenen Disziplinen, mit denen sie seit einigen Jahren kooperiert, auf den Begriff zu bringen, haben sich die Herausgeberinnen des vorliegenden Sammelbandes in Anlehnung an Terhart (2002) für die Metapher der ,fremden Schwestern' entschieden. Das ist aus verschiedenen Gründen eine glückliche Entscheidung. Denn einerseits wird über die Metapher das Assoziationsfeld der familiären Verbundenheit aufgerufen. Kaum eine Metapher ist geeigneter, um ein besonders enges und inniges Verhältnis zwischen Personen oder - in diesem Fall - wissenschaftlichen Disziplinen auszudrücken. Andererseits verweist das Bild der Geschwisterschaft auch auf ein problematisches Verhältnis: Man denke nur an das literarische Motiv der feindlichen Brüder. Glücklicherweise handelt es sich im vorliegenden Fall nicht um Brüder, sondern um Schwestern, die, zumindest wenn man die fiktionale Literatur zu Rate zieht, meist kein so destruktives und aggressives Verhältnis pflegen wie ihre männlichen Pendants. Bei den bösen Schwestern in den bekannten Kinder- und Hausmärchen der Brüder Grimm handelt es sich ja um Stiefschwestern, die eben nicht in einer natürlichen Verbindung zueinander stehen. Weil das Genus der Wissenschaft weiblich ist, ist die metaphorische Bezeichnung wissenschaftlicher Kooperationen als Schwesternschaft sowohl inhaltlich als auch grammatisch ein überzeugender Vorschlag. Attraktiv ist die Metapher aber auch, weil sie weder eine explizite noch eine implizite Rangfolge suggeriert. Geschwister sind zunächst auf einer Hierarchieebene, sie sind gleichberechtigt. Es handelt sich also um eine 
Nähe, die Gleichheit impliziert, ganz im Gegensatz zum metaphorischen Gebrauch der Eltern-Kind-Konstellation. Gleichwohl ist man doch versucht zu fragen: Und wer sind die Eltern der ,fremden Schwestern'? Ein Literaturwissenschaftler (und der bin ich) wäre wohl spontan geneigt zu sagen: ,Die Literaturdidaktik ist eine Tochter der Literaturwissenschaft und der Erziehungswissenschaften. Allerdings scheint mir diese Behauptung zur familiären Genealogie eigentlich zu kurz zu greifen. Es mag womöglich auf die akademische Herausbildung der Fachdidaktik zutreffen, doch geht das Nachdenken über Lehr- und Lernprozesse, insbesondere über das Erlernen von Sprache, Lese- und Schreibfähigkeit, weit vor die akademische Etablierung der Literaturdidaktik und der Literaturwissenschaft zurück.

Umso schwerer wiegt für die Beziehung der Deutschdidaktik zu ihren, Schwestern' das näher spezifizierende Adjektiv: Verweist das ,fremd' auf die Entfremdung der Schwestern? Sind es verloren gegangene oder verborgen gehaltene Geschwister, die jetzt wiederentdeckt werden? Oder handelt es sich um bislang unerkannte und unbekannte Schwestern?

,Fremde Schwestern' der Deutschdidaktik, die in diesem Sammelband zu Wort kommen, sind die Pädagogische Psychologie, die Allgemeine Didaktik und verschiedene Fachdidaktiken. Die Literaturwissenschaft wird nicht genannt. Liegt dieses Nicht-Erwähnen daran, dass sich in der Fachdidaktik der ,szientistische gegen den ,hermeneutischen' Denkrahmen (vgl. Rupp 2016, in diesem Band, S. 50) durchgesetzt hat und deswegen die doch meistenteils dem hermeneutischen Denken verpflichtete Literaturwissenschaft nicht als ,Schwester ' erkannt wird? Sind sich die Literaturwissenschaft und die Literaturdidaktik in den letzten Jahren so fremd geworden, dass sie ihre Gemeinsamkeiten nicht mehr sehen, obwohl Frederking und Henschel die „Deutschdidaktik als transdisziplinäre Wissenschaft" verstehen, „die hermeneutische und empirische Pole in sich vereint"? (Frederking/Henschel, in diesem Band, S. 74) Ohne eine Antwort auf diese Fragen zu geben, könnte es sich lohnen, auch die Literaturwissenschaft in einem interbzw. transdisziplinären Verhältnis zur Literaturdidaktik zu denken. Wenn die Herausgeberinnen in ihrem Vorwort zur Tagung die Herausforderungen einer interdisziplinären Zusammenarbeit benennen und insbesondere die ,je eigene Fachsprache der einzelnen Wissenschaftskulturen oder der jeweiligen disziplinären Theorien und Methoden der einzelnen Partner" ${ }^{\text {"1 }}$ hervorheben, dann ist wohl eine entscheidende Schwierigkeit angesprochen, die auch das Verhältnis von Fachwissenschaft und Fachdidaktik betrifft.

1 Vgl.http://www.didaktikdeutsch.de/data/_uploaded/pdf/FlyerTagungMärzNEU.pdf. Abgerufen am 22.02.2016. 
Im Folgenden möchte ich aus der Sicht eines Literaturwissenschaftlers einige Beobachtungen und Überlegungen zur Deutschdidaktik und ihren ,Geschwisterbeziehungen' anstellen. Meine ersten Anmerkungen bleiben dabei auf einer grundsätzlichen Ebene (Abschnitt 2). Sie gehen auf mein Studium des Lehramts (2000-2006) und auf meine akademische Lehrtätigkeit (seit 2008) zurück. Aus der Perspektive eines Fachwissenschaftlers sichte ich im Folgenden die Beiträge des vorliegenden Sammelbandes und versuche mich an einer vorsichtigen Einschätzung der aktuellen Strömungen innerhalb der Literaturdidaktik (Abschnitt 3). Dass diese Einordnung von außen nur schlaglichtartig und höchst subjektiv ausfallen kann, dürfte auf der Hand liegen. Zum Abschluss meines Beitrags plädiere ich für eine engere Verzahnung von Fachdidaktik und Fachwissenschaft. Dieses Plädoyer halte ich aus der Überzeugung heraus, dass mittlerweile auch das Verhältnis dieser beiden Disziplinen dem zwischen zwei ,fremden Schwestern' gleicht (Abschnitt 4). Wenn man sich als solche (wieder-)entdeckt und sich dann in den mitunter mühsamen Dialog zwischen gleichberechtigen Geschwistern begibt, die sich mit demselben Forschungsgegenstand befassen, ihn aber aus zwei unterschiedlichen Perspektiven betrachten und unterschiedliche Erkenntnisinteressen verfolgen, dann können beide Geschwister dabei voneinander nur profitieren. ${ }^{2}$

\section{Literaturdidaktik aus literaturwissenschaftlicher Sicht}

Die Fachdidaktik halte ich sowohl inhaltlich als auch strukturell für einen äußerst wichtigen Bereich der Germanistik. Inhaltlich weitet die Fachdidaktik den Blick über den literarischen Gegenstand hinaus auf Erwerbs- und Vermittlungsprozesse aus. Damit untersucht sie Prozesse, die mit dem literaturwissenschaftlichen Forschungsgegenstand unmittelbar verbunden sind. Strukturell stützt sie ferner das Universitätsfach Germanistik in ihrer institutionellen Positionierung. Weil die angehenden Deutschlehrerinnen und -lehrer in Deutschland in zwei Phasen ausgebildet werden, sind geisteswissenschaftliche Fächer wie die Germanistik an

2 Zwei Bemerkungen seien noch angefügt: (1) Da ich Literaturwissenschaftler bin, konzentriere ich mich auf das Verhältnis von Literaturdidaktik und Literaturwissenschaft. (2) Meine Beobachtungen, die Sichtung der Beiträge und auch mein Abschlussplädoyer sind notwendigerweise subjektiv gefärbt. Zwar habe ich selbst ein Lehramtsstudium absolviert, doch argumentiere ich aus einer literaturwissenschaftlichen Perspektive. Manche Ausführungen - womöglich die meisten Beobachtungen und Ideen - sind vielleicht weder innovativ noch , auf der Höhe der didaktischen Diskussion. Aber vielleicht ermöglicht der Blick eines Vertreters der ,Schwesterdisziplin' manche NeuPerspektivierung alter Probleme. 
den Universitäten immer noch stark repräsentiert und einflussreich. Ich möchte gewiss nicht behaupten, dass die Germanistik ohne die Lehramtsausbildung zu einem ,Orchideenfach' werden würde, aber weil die Lehramtsstudierenden, die an vielen germanistischen Instituten und Seminaren einen beträchtlichen Anteil der Studierendenschaft ausmachen, eine universitäre Ausbildung erhalten, gehört Germanistik immer noch zu den nachgefragten Fächern. Zwar resultieren aus dieser zweiphasigen Lehrerausbildung einige Probleme, doch halte ich dieses Konzept aus verschiedenen Gründen inhaltlich für sinnvoll. ${ }^{3}$ Nicht nur inhaltliche und strukturelle Argumente, sondern auch politische Aspekte sollten also in einer Perspektive berücksichtigt werden, die die Fachdidaktik aus der Sicht der Fachwissenschaft zu beschreiben versucht. Aus curricularer Sicht sind Literatur- und Sprachdidaktik ein wesentlicher Teil der Ausbildung für das Lehramt Deutsch. Zwar variieren Struktur und Umfang von Universität zu Universität, doch bleibt das Studium der Fachdidaktik essentieller Teil des Studiums für das Schulfach Deutsch. Die Umstellung auf Bachelor und Master hat hier sicherlich die ,Gleichstellung' der Fachdidaktiken mit den Fachwissenschaften wesentlich vorangebracht. Denn in Folge der Bologna-Reform müssen die Pflichtmodule durch grundständige Lehre abgedeckt werden. Allerdings gibt es auch heute noch Prüfungs- und Modulordnungen, die nur verhältnismäßig wenige fachdidaktische Veranstaltungen für das Lehramtsstudium vorsehen.

Insgesamt ist die Fachdidaktik in der universitären Lehre (und mitunter auch in den Forschungsrichtungen und Erkenntnisinteressen) deutlich auf schulische Lehr- und Lernprozesse konzentriert. Das Interesse der Literatur- und Sprachdidaktik an schulischen Lehr-, Lern- und Bildungsprozessen möchte ich an dieser Stelle nicht kritisieren oder gar in Frage stellen. Dass insbesondere die Literaturdidaktik auf die Institution Schule ausgerichtet ist, liegt an der Genese der Fachdidaktik im wissenschaftlichen Kontext, die sich zunächst als ,Handwerkslehre für die angehenden Deutschlehrerinnen und -lehrer verstand (vgl. auch Rupp, in

3 Nicht nur angehende Gymnasiallehrerinnen und -lehrer, sondern auch Oberschul-, Real-, Haupt- oder Grundschullehrerinnen und -lehrer profitieren von dieser zweiphasigen Ausbildung. Deutschlehrerinnen und -lehrer müssen Experten für die deutsche Sprache und Literatur sein. Sie haben die Aufgabe, sowohl als Vermittler kultureller Werte und Normen als auch als Vermittler deklarativen und prozeduralen Wissens zu wirken. Diese Aufgabe ist meines Erachtens nur mit einem bestimmten Bildungsgrad erfüllbar. Das Studium bietet einen Ort, an dem sich Studierende im eigentlichen Sinne des Worts bilden und entwickeln können. Trotz Strukturen, die verstärkt Effizienz und Schnelligkeit forcieren, scheint mir die Universität immer noch der geeignetste Ort für diese Aufgabe zu sein. 
diesem Band, S. 48). ${ }^{4}$ Mittlerweile stehen jedoch nicht mehr primär methodische Fragen im Zentrum der fachdidaktischen Forschung, wie die Beiträge des Sammelbandes eindrucksvoll belegen, sondern eigene Theorie- und Modellbildungen, die sich vielfach im empirischen Rahmen bewegen und sich auf den schulischen Kontext konzentrieren. Auch die Beiträge des vorliegenden Sammelbandes, das lässt sich an dieser Stelle bereits anmerken, fokussieren die Institution Schule. Dabei rückt die Entwicklung der Disziplin ins Zentrum, es werden die Schülerinnen und Schüler und die Lehrerinnen und Lehrer und ihre Ausbildung in den Blick genommen, curriculare Aspekte spielen eine Rolle und auch Fragen des Kompetenzerwerbs und der Kompetenzförderung werden erörtert. Gleichzeitig werden in allen Beiträgen das interdisziplinäre Arbeiten an deutschdidaktischen Fragestellungen und die Gelingensbedingungen interdisziplinärer Arbeit reflektiert.

Allerdings habe ich die Fachdidaktik stets als Teilgebiet der Germanistik verstanden und wahrgenommen, das sich mit Fragen von Erwerb und Vermittlung der germanistischen Gegenstände (allgemein: Sprache und Literatur) befasst. Wenn man diesen Anspruch ernst nimmt, dann weitet sich das Feld aus, in dem didaktische Fragen relevant sind. Literatur- und Sprachdidaktik als Disziplin, die sich mit Erwerb und Vermittlung befasst, kann so auch auf andere Berufsbereiche vorbereiten und (beruflich) relevante Erkenntnisse bieten, die mit didaktischen Frage- und Problemstellungen verbunden sind. Fachdidaktische Veranstaltungen können Studierenden wichtige Einsichten für ihre Aufgabe z. B. im Literaturhaus oder Literaturmuseum, in der Pressestelle, im Verlagswesen, in der Erwachsenenbildung und außerschulischen Kinder- und Jugendbildung, im Theater oder auch als Wissenschaftler bieten. Zudem lassen sich fachwissenschaftliche Fragen in der Interaktion und Kommunikation nur selten von didaktischen Problem- und Hilfestellungen trennen. Anhand von zwei Beispielen möchte ich die Relevanz der Fachdidaktik im außerschulischen Bereich kurz skizzieren.

(1) Das erste Beispiel wähle ich aus einem Bereich, in dem nicht nur Absolventen der Germanistik arbeiten. Wenn in einem Literaturmuseum eine neue Ausstellung geplant wird, dann rückt die Vermittlung des Wissens in den Mittelpunkt. Die Kuratoren und die Museumsmitarbeiterinnen und -mitarbeiter sind mit einer Vielzahl von Fragen konfrontiert: Welche Objekte werden ausgestellt? Wie sollen Exponate präsentiert werden, um die Wirkung zu erzielen, die erzielt werden soll? Welchen Weg sollen die Besucher durch die Ausstellung gehen? Wie umfangreich

4 Für die Sprachdidaktik erkenne ich zwar auch einen Schwerpunkt auf schulische Erwerbs- und Vermittlungsprozesse. Allerdings haben sich in den letzten Jahren einige Zweige der sprachdidaktischen Forschung entwickelt, die sich auf die Erwachsenenbildung, die Sprachdiagnostik oder auch auf Zweit- oder Drittspracherwerb konzentrieren. 
und ausführlich müssen die erklärenden Tafeln zu den Objekten sein? Was soll die Ausstellung eigentlich ,erzählen'? All dies sind genuin didaktische Fragen, mit denen sich Museumsmitarbeiterinnen und Museumsmitarbeiter sowie Kuratoren befassen müssen. Denn sie müssen adressatenbezogen ein Thema, einen Gegenstand oder ein Werk aufbereiten. Sie müssen entscheiden, ob Sachverhalte reduziert, ob und wenn ja, wie sie in welchem Kontext präsentiert werden. Dabei müssen sie freilich mit dem Gegenstand vertraut sein. Sie müssen also ebenso über fachwissenschaftliche wie über fachdidaktische Kompetenz verfügen.

(2) Neben dem Literaturmuseum ist aber auch die Universität ein Arbeitsfeld, in dem fachdidaktische Fragen eine große Rolle spielen. Ich erlaube mir dabei, von persönlichen Erfahrungen auszugehen, um aus meiner Sicht einige wesentliche Aspekte didaktischer Arbeit zu konkretisieren. Dass literaturwissenschaftliche und -didaktische Aspekte in der Seminararbeit oftmals konvergieren, lässt sich allein schon daran zeigen, dass ich meine Lehre selbst didaktisch aufbereite. Diese hochschuldidaktische Arbeit verbindet fachliche Aspekte mit Erkenntnissen der ,Schuldidaktik' und der Erwachsenenbildung. Als Lehrender muss ich komplexe Sachverhalte für Studierende, die sich an einem bestimmten Punkt ihres Studiums befinden, aufbereiten, gegebenenfalls reduzieren oder Zusatzinformationen liefern, damit der Inhalt, die Komplexität des Gegenstands und seine ästhetischliterarische Qualität für die Studierenden nachvollziehbar werden. Ich muss mir Gedanken über die angemessene Art der Vermittlung machen und die relevanten Lernziele für mich und die Studierenden operationalisieren und transparent machen. Dass es sich insbesondere in den unteren Semestern um äußerst heterogene Lerngruppen handelt, kann wohl jeder bestätigen, der in den letzten Jahren an deutschen Universitäten gelehrt hat. Eine heterogene Lerngruppe erfordert mehr als hochschuldidaktische Grundfähigkeiten, wenn man nicht nach dem Motto Franz Moors aus Schillers Räubern verfahren will: „Schwimme, wer schwimmen kann, und wer zu plump ist, geh unter!“ (Schiller 2004, S. 500).

Neben der eigenen hochschuldidaktischen Aufbereitung des Lernstoffs wird die Verbindung von Literaturwissenschaft und Literaturdidaktik auch auf einer Metaebene wichtig. In meinen eigenen universitären Lehrveranstaltungen werde ich vonseiten der Studierenden zunehmend mit der Frage nach dem Sinn der Seminarinhalte konfrontiert. Ohne einen kulturpessimistischen Ton anschlagen zu wollen, möchte ich diese Beobachtung auf ein plakatives Beispiel bringen: Wofür brauche ich die Erzählungen Kafkas, wenn ich doch Grundschullehrer werden 
möchte? ${ }^{\text {‘5 }}$ Diese und ähnliche Fragen höre ich in den letzten Jahren vermehrt, wobei die Autorin bzw. der Autor wechselt. Ich halte es für notwendig, dass Lehrende auf diese Fragen eingehen und so einen Sinnzusammenhang herstellen, der die zweiphasige Lehrerausbildung betrifft (vgl. Dehrmann/Standke 2012, S. 111). Zwar geht es in einem literaturwissenschaftlichen Seminar über Kafkas Erzählungen primär um Kafkas Texte. Gleichzeitig sollen die Studierenden die Fähigkeiten und Fertigkeiten erwerben, um sich eigenständig in ein neues Thema einarbeiten zu können. Es geht also nicht nur um Kafka als literarischen Gegenstand, sondern auch darum, dass die Studierenden die Kompetenz erwerben, sich nach dem Besuch dieses Seminars in andere literaturwissenschaftliche Themen selbstständig einarbeiten zu können. ${ }^{6}$ In einem Schulfach Deutsch, das von Entscheidungen der Kultusministerien auch hinsichtlich der als relevant angesehenen Teilkompetenzen immer wieder stark betroffen ist, scheint mir diese Fähigkeit für angehende Deutschlehrerinnen und -lehrer essentiell. ${ }^{7}$ Nichts ändert sich für Deutschlehrkräfte schneller als die Praxis, weswegen eine rein auf unterrichtliche Anwendung

5 Von einer ähnlichen Erfahrung berichten Mark-Georg Dehrmann und Jan Standke, die ein Themenheft zum Verhältnis von Germanistik und Lehrerausbildung herausgegeben haben (vgl. Dehrmann/Standke 2012, S. 110). Die Autoren kommen dabei auch zu vergleichbaren Schlussfolgerungen. Im Themenheft kommen verschiedene Personen zu Wort, die pointierte Antworten in essayistischer Form auf durchaus komplexe Fragen, die zuvor von Herausgebern gestellt wurden, geben. In vielen Beiträgen werden die strukturelle und die politische Bedeutung der Lehramtsausbildung herausgestellt. Auf inhaltliche Verbindungen zwischen Fachdidaktik und -wissenschaft wird nur zögerlich eingegangen; mitunter wird eine inhaltliche Nähe auch bestritten. Dehrmann und Standke sehen ihren Band vor allem als Anstoß, die Debatte über die Bedeutung der Lehramtsausbildung sowohl vonseiten der Fachwissenschaften als auch vonseiten der Fachdidaktik wiederaufzunehmen (vgl. ebd., S. 117).

6 Bei den angebotenen Seminar- und Vorlesungsthemen spielen Kanonbildungsprozesse eine nicht zu vernachlässigende Rolle. Dies gilt meines Erachtens besonders stark für die Literaturwissenschaft. Ob Kafkas Erzählungen oder ebenso die kinderliterarischen Texte Erich Kästners als Gegenstände des Kompetenzerwerbs für angehende Lehrerinnen und Lehrer geeignet sind, wäre an anderer Stelle ausführlicher zu diskutieren.

7 Ein Lehrer, der Themen unterrichten muss, die er im Studium nicht vertiefend bearbeitet hat und nun Lektürehilfen und andere Unterrichtsmaterialien zur Hand nimmt, wird nicht unabhängig über diese Materialien urteilen können. Er kann somit nicht die gleiche Unterrichtsqualität erreichen wie eine Kollegin, die sich selbstständig in ein Thema einarbeiten kann, und ist zudem weniger flexibel in der konkreten Unterrichtsinteraktion. Ich denke, dass die Fachkompetenz eines Deutschlehrers oder einer Deutschlehrerin hier als regulierende Instanz ins Spiel kommt, um auch die einschlägigen Lektürehilfen in ihrer Qualität sicher beurteilen zu können. 
ausgerichtete Ausbildung ihr Ziel verfehlt (vgl. Nünning 2004, S. 66). Den Lehrenden ist dieser Sachverhalt zumeist klar, insbesondere Studienanfängern muss man diesen Zusammenhang aber erklären. Ich denke, dass dieses Beispiel für jeden Lehrenden an einer Universität unmittelbar einsichtig ist. ${ }^{8}$

\section{Tendenzen der Deutschdidaktik. Über die Beiträge des Sammelbandes}

Die Beiträge des vorliegenden Sammelbandes möchte ich im Folgenden als repräsentative Auswahl betrachten, die Einblick in die neueren Entwicklungen der Deutschdidaktik gibt. Auffällig sind zunächst die Gemeinsamkeiten der Beiträge: Alle kreisen um Fragen der Interdisziplinarität, wobei mitunter auch eine theoretische Fundierung von Interdisziplinarität im Gegensatz zur Transdisziplinarität vorgenommen wird. ${ }^{9}$ Daran anknüpfend greifen einige Beiträge auf die Konzepte des ,Denkstils' und des ,Denkkollektivs' nach Ludwik Fleck zurück (vgl. Schmidt/ Moschner und Pieper/Sâmihăian, in diesem Band). Beinahe alle Beiträge bieten zudem einen Einblick in aktuelle Forschungsprojekte, die interdisziplinär aufgestellt sind. Die Vernetzung der Fachdidaktik mit anderen Disziplinen spielt für alle Beiträge eine große Rolle und wird einige Male als strukturell und (hochschul-)politisch wichtig erachtet. Insbesondere an diesen Stellen wird aber auch immer wieder deutlich, dass auch literaturwissenschaftliche Modellierungen und methodische Werkzeuge von Bedeutung sind. Einer literaturwissenschaftlichen Perspektive wird in den Beiträgen des Sammelbandes also durchaus Rechnung

8 Der Einschätzung von Juliane Köster kann ich daher nicht zustimmen, wenn sie eine grundsätzliche Differenz zwischen Literaturdidaktik und Literaturwissenschaft darin sieht, dass die Lernenden und die Erwerbsprozesse der Lernenden in der Literaturwissenschaft (angeblich) keine Rolle spielen: „Anders verhält es sich in der Literaturwissenschaft. Interpretation von Literatur bzw. der reflexive Umgang mit ihr ist ihr Kerngeschäft. Sie tut dies theoriegeleitet im Kontext aktuellen Erkenntnisinteresses. Die akademische Lehre ist dem nachgeordnet. Die Frage, wie Literaturstudierende zu kompetenten Interpretinnen und Interpreten werden, d. h. auch die beste Expertise erwerben, beschäftigt die Literaturwissenschaft allenfalls am Rande“ (Köster 2015, S. 14). Man täte gut daran, an dieser Stelle zwischen literaturwissenschaftlichen Forschungen und literaturwissenschaftlichen Seminaren zu unterscheiden. Verallgemeinernd von „der“ Literaturwissenschaft zu sprechen, um sie „der“ Literaturdidaktik gegenüberzustellen, halte ich für problematisch, wenngleich ich selbst in meiner Einleitung diese sprachliche Verallgemeinerung verwendet habe.

9 Freilich ist diese thematische Fokussierung (auch) dem Schwerpunkt des vorliegenden Sammelbandes geschuldet. 
getragen. Dass die Literaturdidaktik die Literaturwissenschaft verleugnen würde, kann nicht behauptet werden. ${ }^{10}$ Gleichzeitig ist eine weitere Gemeinsamkeit zwischen den einzelnen Beiträgen auszumachen: Die vorgestellten Projekte sind bis auf wenige Ausnahmen - empirisch ausgerichtet. Die empirische Wende der Deutschdidaktik, die in dem Überblicksartikel von Gerhard Rupp dargestellt wird, schlägt sich in den Beiträgen deutlich nieder. Rupp stellt zunächst eine Phasengliederung der Entwicklung der Deutschdidaktik vor, um die einzelnen Phasen kurz zu charakterisieren und mit einer knappen Bewertung der Forschungsentwicklung zu schließen. Er sieht „die Zukunft“ der Deutschdidaktik „Zweifellos“ in der „Fortsetzung interdisziplinärer und auch internationaler Verbundforschung“ (Rupp, in diesem Band, S. 62). Was bei Rupps Ausführungen ferner indirekt ablesbar wird: An der Art, wie Literaturdidaktik betrieben wird, lässt sich ein ,Zeitgeist der Literaturvorstellung feststellen. Auf welche implizite Literaturvorstellung nun die empirische Wende der Deutschdidaktik verweist, müsste an anderer Stelle wohl systematischer durchdacht werden.

Im Zentrum des Beitrags von Iris Winkler, Matthias Heinrich, Astrid Fischer und Ulrike Krause steht ein Projekt zum fächerübergreifenden Lernen in der Ausbildung von Mathematik- und Deutschstudierenden: Im Projekt wird untersucht, „inwieweit fächerübergreifendes Lernen in der Mathematik- und Deutschdidaktik zur Förderung fachdidaktischer Kompetenzen bei Lehramtsstudierenden beider Fächer beiträgt" (Winkler et al., in diesem Band, S. 181). Ausgehend von der Beobachtung, dass Studierende der Fächer Mathematik und Deutsch oftmals gegenläufige und „simplifizierende Vorstellungen vom jeweils anderen Fach haben“ (ebd., S. 184), soll über die Spiegelung ein neuer Blick auf das jeweilige Unterrichtsfach ermöglicht werden. Ohne ausführlich auf das Fragedesign einzugehen, möchte ich den qualitativen Teil der Erhebung hervorheben. Hier wurden Deutschstudierende und Studierende der Mathematik nach ihren impliziten Annahmen über das jeweils andere Fach befragt. Es geht um die „Überzeugungen“, „die sich in der Regel bei Lernenden während der Schulzeit in Bezug auf das jeweilige Fach gebildet haben.“ (ebd., S. 188) Aufgrund der Befragung können „Unterrichtsmerkmale in den Vordergrund“ rücken, „die aus fachimmanenter Perspektive tradiert und kaum infrage gestellt werden" (ebd., S. 189). So kritisieren Mathematikstudierende beispielsweise die Offenheit des Deutschunterrichts, da sie zur Verunsicherung über ,richtig' und ,falsch' und schließlich auch zur Intransparenz der Bewertung

10 Dies gilt für die einzelnen Beiträge freilich in unterschiedlichem Maße, da in den vorgestellten Projekten verschiedene Erkenntnisinteressen verfolgt werden, die nicht immer offensichtlich eine Schnittstelle zur Literaturwissenschaft bieten. 
führe. Die systematische Mehrdeutigkeit literarischer Texte wird als interpretative Beliebigkeit und als ,Gefahr" wahrgenommen. Die Deutschstudierenden bemängeln am Mathematikunterricht hingegen die vermeintliche Begrenztheit des Faches: Mathematikunterricht sei Auswendiglernen von Regeln. Dass diese Auffassung ihrerseits begrenzt ist, zeigt sich daran, dass wesentliche Bereiche der Vektorgeometrie oder Analysis eben nicht mit Auswendiglernen von Regeln zu verstehen sind. Der Perspektivwechsel, so das Fazit der Studie, sensibilisiert für mögliche Probleme, die im konkreten Unterricht entstehen, weil epistemologische Vorannahmen existieren, die dem jeweiligen Gegenstand nicht immer gerecht werden. Das hier vorgestellte Projekt ist ein sehr gutes Beispiel für eine von neuen Perspektiven profitierende Fachdidaktik, die gleichzeitig sowohl theoretische Modellierungen (Bedeutung epistemologischer Vorannahmen bei Lernenden und Lehrenden) als auch unterrichtspraktische Konsequenzen in den Blick nimmt. Dass insbesondere die systematische Mehrdeutigkeit literarischer Texte von Studierenden der Mathematik als ,Problem' artikuliert wird, ist aus meiner Sicht ein wichtiges Ergebnis, das ernst genommen werden muss.

Mit dem Nutzen interdisziplinärer Arbeit in der Promotionsphase befasst sich hingegen der Artikel von Frederike Schmidt und Barbara Moschner. Dabei gehen Schmidt und Moschner zunächst auf die allgemeinen Chancen von Interdisziplinarität ein. Ausgehend von der Erkenntnistheorie Ludwik Flecks, dessen Arbeiten bislang vor allem in der Wissenschafts- und Ideengeschichte rezipiert wurden, stellen die Autorinnen die Ausgangsthese auf, dass in der gemeinsamen Forschungsarbeit die jeweiligen Disziplinen von den kooperierenden Disziplinen profitieren können. (Schmidt/Moschner, in diesem Band, S. 202) Flecks Konzept des ,Denkstils 'ist dabei für die Argumentation leitend. Laut Fleck beeinflusst unser Denkstil nicht nur die Perspektive, sondern auch die Wahrnehmung und den hypothetischen Möglichkeitshorizont des wissenschaftlichen Arbeitens. Interdisziplinäres Arbeiten ist, in aller Kürze, das Aushandeln zwischen unterschiedlichen Denkstilen verschiedener ,Denkkollektive. Wenn Interdisziplinarität glückt, dann können die unterschiedlichen Denkstile und die einzelnen Akteure der Denkkollektive voneinander profitieren, weil ihre Denk-, Wahrnehmungs- und Handlungsmuster ergänzt und erweitert werden. Am Beispiel des Oldenburger Promotionsprogramms „Prozesse fachdidaktischer Strukturierung " (ProfaS) stellen die Autorinnen interdisziplinäres Arbeiten aus der Sicht einer Promotionsbetreuerin und einer Doktorandin dar. In dem Artikel, der eine anschauliche Verknüpfung von Erfahrungsbericht und theoretischer Untermauerung bietet, wird die Auffassung vertreten, dass auch die Vernetzung der unterschiedlichen Fachdidaktiken interdisziplinäres Arbeiten darstellt. Gleichwohl wird auch hervorgehoben, dass „, $[\mathrm{t}]$ rotz der Möglichkeiten 
zum produktiven Dialog zwischen den Fachkulturen [...] die Kernkompetenzen des eigenen Faches in der interdisziplinären Arbeit bewahrt werden müssen“ (ebd., S. 216). Dass unproduktive Konfrontationen in der interdisziplinären Arbeit aufgelöst werden konnten und man von den fachfremden Theorien und Modellen profitiert hat, ist der Grundtenor des Erfahrungsberichts über das Promotionsprogramm. Als Alternative zur Metapher der ,fremden Schwestern' führen Schmidt und Moschner die Metapher der „kritischen Freundinnen“ (ebd., S. 219) ein, um das Verhältnis zwischen den beteiligten Fächern zu beschreiben. Welcher heuristische Mehrwert hier gegenüber der Leitmetapher des Sammelbandes besteht, wird allerdings nicht erläutert.

Katrin Böhme, Petra Stanat und Michael Becker-Mrotzek geben in ihrem Artikel Einblick in die Arbeit des Instituts zur Qualitätsentwicklung im Bildungswesen (IQB). Im Zentrum des Beitrags steht die Darstellung interdisziplinärer Zusammenarbeit am Beispiel der Entwicklung integrativer Kompetenzstufenmodelle in der Sekundarstufe I des Deutschunterrichts. Um Testaufgaben zu modellieren und den inhaltlich definierten Kompetenzniveaus zuzuordnen, muss inter- und transdisziplinär gehandelt werden. Denn Deutschdidaktiker gehen hier in einen Abstimmungsprozess mit der Bildungspolitik und der Schulpraxis. In ihrem Artikel machen Böhme und Becker-Mrotzek darauf aufmerksam, dass die empirische Wende der Deutschdidaktik auch aus der Steuerung des deutschen Bildungssystems resultiert, das sich verstärkt auf empirisch erhobene Daten stützt.

Auch Volker Frederking und Sofie Henschel stellen ein aktuelles empirisches Forschungsprojekt vor. Zunächst konstatieren sie, dass interdisziplinäre Kooperationen in der Literaturdidaktik immer noch eine Ausnahme seien, wofür sie die "hermeneutische Verwurzelung" (Frederking/Henschel, in diesem Band, S. 72) als einen Grund anführen. Gleichzeitig werden Interdisziplinarität und empirische Ausrichtung in einem kausalen Zusammenhang gesehen - empirisches Arbeiten wird implizit als Voraussetzung für Interdisziplinarität gesetzt (vgl. ebd., S. 72). Frederking und Henschel zeigen überdies die Relevanz der Empirie auch an der Entwicklung anderer Fächer auf. Dass sich Psychologie und Bildungswissenschaften in den letzten Jahren zu empirischen Fachrichtungen entwickelt haben, lässt sich nicht bestreiten, und auch die Literaturdidaktik scheint diesen Weg zu gehen. Ihr wird in der Gruppe der ,empirisierten 'Wissenschaften aber eine Sonderrolle zuteil, denn die Literaturdidaktik vereint "hermeneutische und empirische Pole“ (ebd., S. 74). Auch wenn Frederking und Henschel die hermeneutische Tradition goutieren, so sind sie wohl dem, empirischen Pol' zuzurechnen. Dies zeigt sich am ambitionierten Projekt „Literarische Urteils- und Verstehenskompetenz“ (LUK). Im Kern geht es dabei um die Erforschung literarischer Verstehenskompetenz, 
wobei u. a. die Modellierung der literarischen Verstehenskompetenz die Projektgruppe vor beträchtliche literaturtheoretische Herausforderungen stellte. Weil literarische Texte eine systematische Mehrdeutigkeit besitzen, man aber nicht von einer interpretativen Beliebigkeit literarischer Texte ausgehen wollte, griff die Projektgruppe auf literaturwissenschaftliche Positionen zurück, die „verbindliche Bedeutungszuschreibungen im Zusammenhang mit literarischen Texten" und „klare und distinktive argumentative Begründungen von Verstehensurteilen“ (ebd., S. 77) vertreten. Um einen operationalisierbaren Teil literarischer Verstehenskompetenz zu extrapolieren, wird dabei an Umberto Ecos Konzept der intentio operis angeknüpft und mit Karl Poppers Falsifikationsprinzip kombiniert; die spezifische Literarizität fiktionaler Texte wird also theoretisch-methodisch ernst genommen. Obwohl Frederking und Henschel am Anfang ihres Beitrags die von ihnen genannten beiden Pole als gleichberechtigt anerkennen, irritiert das Abschlussplädoyer:

Tatsächlich gibt es in der Deutschdidaktik [!] - anders als in der empirischen Bildungswissenschaft - leider eine Tendenz, ideologisch zu polemisieren statt rational und sachlich zu argumentieren. Das verfehlt die Standards eines wissenschaftlichen Diskurses innerhalb einer Disziplin und erschwert den interdisziplinären Diskurs mit anderen Disziplinen. Mittelfristig könnte dadurch auch der Status der Deutschdidaktik als Wissenschaft Schaden nehmen. (ebd., S. 91)

$\mathrm{Ob}$ in der Deutschdidaktik tatsächlich immer noch gegen empirische und interdisziplinäre Ansätze „ideologisch [...] polemisier[t] “ wird und so eine rational-sachliche Argumentation erschwert wird, wie Frederking und Henschel vorbringen, kann ich nicht beurteilen. Das ideologische ,Grabenkampf-' und ,Katastrophen-Szenario, das im obigen Zitat heraufbeschworen wird, kann ich mit Blick auf die Beiträge des Sammelbandes allerdings nicht teilen.

Thomas Lindauer, Claudia Schmellentin und Anne Beerenwinkel befassen sich mit sprachbewusstem Naturwissenschaftsunterricht und geben einen „Werkstattbericht zu einem transdisziplinären Entwicklungsprojekt“. Dabei fokussiert auch dieser Beitrag die Institution Schule und die Frage, wie Bildungsprozesse in ihr wirken. Die enge Bezugnahme zeigt sich beispielsweise an den Ergebnissen des vorgestellten Projekts (u. a. wurden Handreichungen für sprachbewussten Fachunterricht erstellt). Für die Fachdidaktik prägen die Autoren den Begriff der ,inhärenten Interdisziplinarität' (vgl. Lindauer et al., in diesem Band, S. 226), um deutlich zu machen, dass sich Fachdidaktiken zwar am Wissen und an den Methoden anderer Disziplinen orientieren, dennoch aber eine eigenständige akademische Disziplin sind. Fachdidaktische Forschung kann in hohem Maße inter- und transdisziplinär ausgerichtet sein, weil es zwischen den Fachdidaktiken Schnittmengen 
gibt (vgl. ebd., S. 229). Lindauer et al. untersuchen in ihrer Studie die Verbindung von fachlichem Lernen und sprachlicher Kompetenz. Ausgangspunkt sind die Ergebnisse der PISA-Studie, aus denen hervorgeht, dass eine Korrelation zwischen Sprachschwäche und Schwächen beim Fachwissen besteht. In einer gewissen Weise besteht hier eine Verbindung zur Erforschung der Hörkompetenz (siehe den Artikel von Behrens und Weirich). Zwar gehen Lindauer et al. auf die mündliche Vermittlung als eine Dimension der Sprachkompetenz ein, doch modellieren sie in ihrem Artikel sprachliche Kompetenz eher als textuell-schriftliche Kompetenz. Dass naturwissenschaftliche Texte, die entweder von Schülerinnen und Schülern produziert werden oder als Lernmaterial dienen, anders aufgebaut sind als geisteswissenschaftliche Texte, ist evident. Aber vielleicht ist neben der Fachwortdichte ein weiteres Differenzkriterium, dass geisteswissenschaftliche Schultexte oftmals narrativ strukturiert sind. Unabhängig von diesem möglichen Unterschied geht es Lindauer et al. grundsätzlich nicht darum, den Schülerinnen und Schülern ein überfachlich geprägtes bildungssprachliches Register (ebd., S. 232) zugänglich zu machen. Vielmehr erachten sie die Sprachförderung als Gegenstand aller Unterrichtsfächer. Das ist ein ambitioniertes Ziel, muss die Sprachförderung im naturwissenschaftlichen Unterricht doch notwendigerweise auf Kosten der Vermittlung anderer Inhalte gehen. Gleichwohl werden die wesentlichen Aufgaben zur Förderung der Sprachkompetenz weiterhin beim Deutschunterricht gesehen:

Gedacht wird dabei an eine ,Arbeitsteilung' zwischen Deutschunterricht und den anderen Fächern, die darin besteht, dass im Deutschunterricht die relevanten sprachlichen Strategien kleinschrittig eingeführt werden, da dort der Ort und die Zeit ist, diese Strategien vertiefend zu reflektieren. Aufgabe der anderen Fächer ist es dann, die für den eigenen Fachkontext nötigen Sprachstrategien aufzugreifen und die Lernenden sprachlich so zu unterstützen, dass sie dem fachlichen Unterricht folgen können. (ebd., S. 239)

Mit dieser Forderung wird eine Aufgabe des Deutschunterrichts (Sprachförderung) deutlich betont, was zu Lasten anderer Aufgaben gehen könnte.

Von einer ähnlichen Seite nähern sich Ulrike Behrens und Sebastian Weirich einem vergleichbaren Problem wie Lindauer, Schmellentin und Beerenwinkel. Behrens und Weirich berichten aus einer interdisziplinären Projektgruppe, die sich mit dem Hörverstehen bei Grundschülerinnen und Grundschülern befasst. Die Untersuchung der Zuhörkompetenz ist ein vielversprechendes Forschungsfeld. So „stecken Modelle zur Zuhörkompetenz nach wie vor in den Kinderschuhen“ (Behrens/Weirich, in diesem Band, S. 146), obwohl Zuhörkompetenz im Schulunterricht von zentraler Bedeutung ist. Der Beitrag berichtet also von einem Desiderat, dessen Erforschung auch für die Unterrichtspraxis von großer Relevanz sein dürfte. Allerdings verfolgen Behrens und Weirich meines Erachtens eher 
allgemein-didaktische Fragen. Dies zeigt sich am Testdesign. Die Aufgaben, die die Zuhörkompetenz prüfen, beziehen sich auf zwei Textsorten: literarische Texte und Radiosendungen zu Sachthemen. Im Zentrum steht dabei die Abprüfbarkeit von zwei Informationstypen: (1) Sachinformationen und (2) Informationen, die über Intonation und Prosodie vermittelt werden. Die von den Autoren vorgestellten Beispielfragen beziehen sich auf ein Kinderbuch, also auf einen literarischen Text, der vor allem dazu dient, die prosodischen Elemente zu prüfen. Zwar werden auch Inhaltsfragen angeführt, doch scheinen die paraverbalen Items, die sich auf die prosodische Information stützen, zentral. Die Überlegungen zur Bedeutung von Intonation und emotionaler Färbung des Gesagten, die auch auf die Textaussage einwirken, sind durchaus überzeugend. Dass aber ein Literaturwissenschaftler bei einer Studie, die sich mit Zuhörkompetenz vor allem in pragmatischer Sicht befasst, die ästhetische Dimension als unterrepräsentiert beklagen muss, dürfte wenig überraschen. Insgesamt scheint mir hier das Testdesign für pragmatische Texte (also: Sachtexte) besser geeignet als für literarische Texte. Schlaglichtartig zeigt sich dies an zwei Aspekten. Bei dem dargestellten Beispiel handelt es sich um einen kurzen Textauszug aus dem Kinderbuch Die wilde Sofie. Unter anderem musste dafür der Titel der Erzählung verändert werden, „um die Zuhörenden nicht zu verwirren“ (ebd., S. 143). Den Grundschülerinnen und Grundschülern wurde lediglich eine kurze Passage aus dem Mittelteil des Buches präsentiert. Hier wird somit ein kinderliterarischer Text ,passend gemacht', um das abzuprüfen, was man abprüfen will, ungeachtet der Tatsache, dass mit dem Titelwechsel und dem Isolieren eines Textauszugs sich die Textaussage verändert. Zwar werden diese Probleme im Beitrag durchaus erwähnt, doch werden mögliche Bedenken zugunsten der Durchführbarkeit in Kauf genommen. Diesen Schritt kann man auch als Literaturwissenschaftler nachvollziehen. Allerdings irritiert aus literaturwissenschaftlicher Perspektive die anschließende Modellierung des Erwartungshorizonts der Testfragen, wenn klar definiert wird: „Eine Geschichte zu verstehen bedeutet, auf Rezipientenseite eine mentale Repräsentation der Textinhalte zu konstruieren, die der Intention aufseiten des Autors entspricht" (ebd., S. 145). Für pragmatische Texte mag diese Definition gelten, das spezifisch Literarische gerät so jedoch aus dem Blick. Statt auf die Autorintention zu zielen, wäre es doch angebrachter, die Textaussage in den Mittelpunkt zu rücken (wie es beispielsweise Frederking und Henschel unter Rekurs auf die intentio operis machen). Vielmehr erschweren sich die Autoren mit dieser Definition die Operationalisierbarkeit des Erwartungshorizonts. Denn eigentlich müssten sie Lukas Hartmann und Susann Opel-Götz fragen, welche Intentionen sie bei der Verfertigung verfolgt haben - und ob die 
Textproduzenten das gesamte Deutungsspektrum ihres Textes bei der Produktion intendiert haben (können), sei dahingestellt. ${ }^{11}$

Erleidet der literarische Gegenstand eine Suspendierung im Deutschunterricht? Tatsächlich verliert Literatur im konkreten Unterricht an Geltung, der Lerner rückt hingegen als subjektiv interpretierender Leser ins Zentrum des Unterrichts (vgl. Pieper 2015). Diese unterrichtspraktische Diagnose wird ergänzt von den Ergebnissen des Beitrags von Irene Pieper und Florentina Sâmihăian, die sich mit den Curricula unterschiedlicher Nationen beschäftigt haben. Im Vergleich der Curricula der Klassenstufen 7 und 12 ist das, linguistische Paradigma' (, linguistic paradigm') deutlich bestimmend, das Paradigma der ,Persönlichkeitsentwicklung ‘ (,personal growth paradigm ') nimmt von Klassenstufe 7 zu 12 signifikant ab. Die staatlichen Vorgaben hinsichtlich der zu vermittelnden Kompetenzen betonen also die Sachkompetenz, während die persönliche Entwicklung der Lerner in den Hintergrund rückt. Dennoch konstatieren die Autorinnen: „We have to mention that not all the analysed curricula refer to this dimension. This means that teachers have large space for choosing in this matter and that their responsibility for the decisions is high" (Pieper/Sâmihăian, in diesem Band, S. 171), womit gewiss eine Erklärung gegeben wird, warum der Lerner im konkreten Unterricht im Zentrum steht. Auch im Beitrag von Pieper und Sâmihăian hat die empirische Wende der Deutschdidaktik ihren Niederschlag gefunden. Und auch hier wird das Konzept des ,Denkstils' (Fleck) in Anschlag gebracht, um die Probleme interdisziplinärer und internationaler Forschung zu benennen. Bietet die Empirie vielleicht eine Art Lingua franca, um mit anderen Disziplinen zu kooperieren? Ist die empirische Wende in der Deutschdidaktik also die Grundlage für interdisziplinäre Kooperationen oder ist sie nur die Konsequenz aus Interdisziplinarisierung und Internationalisierung?

Welche Schlüsse sind nun aus dieser Zusammenschau deutschdidaktischer Forschungen zu ziehen?

Juliane Köster gibt in einer Zusammenfassung der Tagung Interpretationskulturen (2015), die sich um den transdisziplinären Brückenschlag zwischen Literaturdidaktik und -wissenschaft bemüht, aus literaturdidaktischer Sicht zu bedenken:

Allem voran erscheint die Wiederaufnahme resp. Fortführung der Theorie-Empirie-Debatte in der Literaturdidaktik dringend erforderlich. Das gilt umso mehr, als die Polarisierung

11 Hartmann ist der Autor des Kinderbuchs Die wilde Sofie. Dass die Illustrationen offensichtlich keine Rolle spielen, für die Susann Opel-Götz verantwortlich zeichnet, dürfte dem Testdesign und dem Erkenntnisinteresse geschuldet sein. Schließlich geht es um das Hörverstehen. 
empirischer und hermeneutischer Orientierungen wenig produktiv erscheint und mit dem Verzicht auf fruchtbare Wechselwirkungen und Kooperationen einhergeht. (Köster 2015, S. 20)

In den vorliegenden Beiträgen dieses Sammelbandes wird die empirische Orientierung i. d. R. nicht gegen die Hermeneutik ausgespielt. Ganz im Gegenteil: Die vorliegenden Beiträge belegen geradezu die Suche nach ,fruchtbaren Wechselwirkungen und Kooperationen'. Diese Kooperationen sind zumeist empirisch fundiert. Dass die hermeneutische Tradition der Literaturdidaktik eher unterrepräsentiert ist, mag dabei durchaus als Beleg für die empirische Wende der Deutschdidaktik genommen werden.

\section{Literaturwissenschaft und Literaturdidaktik als ,fremde Schwestern'}

Vielleicht verführt die empirische Wende tatsächlich zu einer Trennung von Gegenstandsorientierung und methodischer Vermittlung. Dass die Empirie-Orientierung für die Konstitution der Deutschdidaktik als eigenes Fach in Abgrenzung zu ihren Bezugswissenschaften nicht die möglicherweise erhoffte Wirkung hat, hat Iris Winkler an anderer Stelle erörtert (vgl. Winkler 2012). Gleichwohl hat die Empirie-Orientierung zu einer Neuausrichtung des Faches geführt. Dabei könnte dieses neue Paradigma in der Deutschdidaktik vielleicht als Lingua fran$c a$ verstanden werden, um unterschiedliche ,Denkstile zu überwinden und in Kooperation und Austausch mit anderen Wissenschaftsdisziplinen zu kommen. Trotz dieses Paradigmenwechsels und der Betonung des Kompetenzerwerbs darf der ,inhaltliche Bildungswert' des Gegenstands nicht verloren gehen. Thomas Zabka betont daher:

Literarische Texte dürfen keine beliebigen Gegenstände des Kompetenzerwerbs sein, sondern sollen nach Maßgabe ihres inhaltlichen Bildungswerts ausgewählt werden. Sinnvolles Inhaltslernen darf nicht als eine spätere, außerschulische Frucht des Kompetenzerwerbs versprochen werden, sondern muss diesen jederzeit begleiten. (Zabka 2012, S. 108)

Dieses Theorie-Praxis-Problem manifestiert sich für die angehenden Lehrerinnen und Lehrer dann auf einer anderen Ebene in der zweiten Phase der Lehramtsausbildung. Der sogenannte ,Praxisschock' verführt manche zu der Argumentation, dass Theoriebildung und hervorragende Theoriekenntnis nicht ausreichend sind, um eine gute Lehrerin oder ein guter Lehrer zu sein. Freilich zieht gute Theoriekenntnis nicht notwendigerweise gute Praxis nach sich, dem ist kaum zu widersprechen. Ohne Zweifel aber ist das Wissen um theoretische Modellierungen grundlegend für die Fähigkeit zur Reflexion wie auch für Innovation. 
Auch wenn in den vorliegenden Beiträgen der Schwerpunkt auf einer interdisziplinären Arbeit liegt, zu der eine Kombination aus fachwissenschaftlicher und -didaktischer Perspektive nicht zählt, möchte ich dennoch für eine enge Verzahnung von Fachwissenschaft und Fachdidaktik plädieren. Denn mein Eindruck ist durchaus, dass sich die Deutschdidaktik in ihren Erkenntnisinteressen und Untersuchungsmethoden von der Literaturwissenschaft entfernt hat. In welchen Bereichen könnten Literaturdidaktik und Literaturwissenschaften aber voneinander profitieren? Ohne den Anspruch auf Vollständigkeit erheben zu wollen, dürfte die Kooperation zu drei Themenbereichen sinnvoll sein: (1) Ich denke, dass aus einer engeren Zusammenarbeit zwischen Literaturwissenschaften und Literaturdidaktik auch eine Öffnung fachdidaktischer Fragen jenseits des Schulkontexts ermöglicht werden könnte. (2) Während sich Literaturwissenschaftler i. d. R. nicht für adressatenbezogene Fragen interessieren, sind die Adressatenbezogenheit und die Frage nach literarischer Komplexität (und worin diese besteht) zentrale Forschungsbereiche der Literaturdidaktik. Auch hier wäre ein Austausch sicherlich insbesondere für Literaturwissenschaftler - ertragreich. (3) Daran anschließend wäre auch das Problem, die systematische Mehrdeutigkeit zu modellieren, ein gemeinsames Thema. Insbesondere der Austausch zwischen empirischen und hermeneutischen Ansätzen (hier Literaturdidaktik, dort Literaturwissenschaft) dürfte bei einem aufgeschlossenen Austausch für beide Seiten fruchtbar sein. Dass die systematische Mehrdeutigkeit literarischer Texte insbesondere bei der Modellierung der Prüfungsitems in vielen Projekten, von denen die Beiträge berichten, zum Thema und mitunter zum Problem wird, verweist meines Erachtens einerseits auf die Dringlichkeit einer solchen Kooperation, andererseits verweist es auf die zunehmende Distanz zwischen Literaturwissenschaft und Literaturdidaktik. Die letzte Feststellung möchte ich nicht als Kritik verstanden wissen. Insbesondere bei der Auseinandersetzung mit der systematischen Mehrdeutigkeit literarischer Texte bietet die Kooperation zwischen Literaturdidaktik und -wissenschaft Potenziale, mittlerweile eingefahrene oder sich einfahrende Strukturen zu hinterfragen und neue Wege zu beschreiten. Zwar haben nach der PISA-Studie Sachtexte im Deutschunterricht einen größeren Stellenwert erhalten, doch für den Erwerb wichtiger Kompetenzen, wie beispielsweise ästhetische Verstehenskompetenz oder Symbolverstehen, erscheinen mir literarische Texte immer noch die angemessenen Gegenstände zu sein. Dass Literatur für viele Schülerinnen und Schüler keinen ,Lebensweltbezug' mehr habe, möchte ich als Argument nicht gelten lassen. Alteritäts- und Fremdheitserfahrungen ermöglichen doch meistens überhaupt die intersubjektive Betrachtung - auch dies müsste aber differenzierter ausgeführt werden. Vielleicht ist mittlerweile der Grund bereitet für eine produktive und 
gleichberechtigte Kooperation zwischen Literaturdidaktik und -wissenschaft. Dabei ist die Distanz zwischen Literaturwissenschaft und -didaktik möglicherweise die Gelingensbedingung für eine produktive Kooperation. Denn manchmal benötigt man auch von seinen Geschwistern ein wenig Distanz, um sich wieder einander annähern zu können und vom Wissen der ,fremd gewordenen Schwester ${ }^{\text {zu }}$ profitieren.

\section{Literatur}

Behrens, Ulrike/Weirich, Sebastian (2016): It takes a whole village... Interdisziplinäre Perspektiven bei der Entwicklung eines Testverfahrens zum Zuhören. In: Winkler, Iris/Schmidt, Frederike (Hrsg.): Interdisziplinäre Forschung in der Deutschdidaktik. „Fremde Schwestern“ im Dialog. Frankfurt a. M.: Lang, S. $133-152$.

Böhme, Katrin/Stanat, Petra/Becker-Mrotzek, Michael (2016): Die Entwicklung integrierter Kompetenzstufenmodelle als Ergebnis interdisziplinärer Kooperation. In: Winkler, Iris/Schmidt, Frederike (Hrsg.): Interdisziplinäre Forschung in der Deutschdidaktik. „Fremde Schwestern“ im Dialog. Frankfurt a. M.: Lang, S. 101-131.

Dehrmann, Mark-Georg/Standke, Jan (2012): Germanistik und Lehrerbildung Einleitung. In: Mitteilungen des Deutschen Germanistenverbandes. Jg. 59. H. 2, S. 107-117.

Frederking, Volker/Henschel, Sofie (2016): Interdisziplinäre Forschung in der Deutschdidaktik am Beispiel des Projekts, Literarästhetische Urteils- und Verstehenskompetenz' (LUK). In: Winkler, Iris/Schmidt, Frederike (Hrsg.): Interdisziplinäre Forschung in der Deutschdidaktik. „Fremde Schwestern“ im Dialog. Frankfurt a. M.: Lang, S. 71-99.

Köster, Juliane (2015): Die Tagung aus literaturdidaktischer Beobachterperspektive. In: Lessing-Sattari, Marie/Löhden, Maike/Meissner, Almuth/Wieser, Dorothee (Hrsg.): Interpretationskulturen. Literaturdidaktik und Literaturwissenschaft im Dialog über Theorie und Praxis des Interpretierens. Frankfurt a. M. u. a.: Lang, S. 11-35.

Lindauer, Thomas/Schmellentin, Claudia/Beerenwinkel, Anne (2016): Sprachbewusster Naturwissenschafts-Unterricht - Werkstattbericht zu einem transdisziplinären Entwicklungsprojekt. In: Winkler, Iris/Schmidt, Frederike (Hrsg.): Interdisziplinäre Forschung in der Deutschdidaktik. „Fremde Schwestern“ im Dialog. Frankfurt a. M.: Lang, S. 225-246.

Nünning, Ansgar (2004): Es geht immer auch anders, oder: Unzeitgemäßes Plädoyer für den Nutzen (und die ,Praxisrelevanz') literaturwissenschaftlicher Theorien, Modelle und Methoden für die Literaturdidaktik und den Literaturunterricht. 
In: Bredella, Lothar/Delanoy, Werner/Surkamp, Carola (Hrsg.): Literaturdidaktik im Dialog. Tübingen: Günter Narr, S. 65-97.

Pieper, Irene (2015): Zur spezifischen Rahmung des Interpretierens in der Schule: Willkommen und Abschied als Unterrichtsgegenstand in der Mittelstufe. In: Lessing-Sattari, Marie/Löhden, Maike/Meissner, Almuth/Wieser, Dorothee (Hrsg.): Interpretationskulturen. Literaturdidaktik und Literaturwissenschaft im Dialog über Theorie und Praxis des Interpretierens. Frankfurt a. M. u. a.: Lang, S. 185-218.

Pieper, Irene/Sâmihăian, Florentina (2016): International Research Cooperation in What Used to Be Called Mother Tongue Education. The Example of LiFT2. In: Winkler, Iris/Schmidt, Frederike (Hrsg.): Interdisziplinäre Forschung in der Deutschdidaktik. „Fremde Schwestern“ im Dialog. Frankfurt a. M.: Lang, S. $153-180$.

Rupp, Gerhard (2016): Fremde und vertraute Schwestern - Konstellationen interdisziplinärer Forschung in der Deutschdidaktik seit den 90er Jahren. In: Winkler, Iris/Schmidt, Frederike (Hrsg.): Interdisziplinäre Forschung in der Deutschdidaktik. „Fremde Schwestern“ im Dialog. Frankfurt a. M.: Lang, S. 43-69.

Schiller, Friedrich (2004) [1781]: Die Räuber. In: Ders.: Sämtliche Werke. Bd. I: Gedichte und Dramen. Hrsg. v. Albert Meier. München und Wien: Hanser, S. 481-618.

Schmidt, Frederike/Moschner, Barbara (2016): „Fremde Schwestern“ und „kritische Freundinnen“ - Lehren und Lernen in einem interdisziplinären Promotionsprogramm in der Lehrerbildung. In: Winkler, Iris/Schmidt, Frederike (Hrsg.): Interdisziplinäre Forschung in der Deutschdidaktik. „Fremde Schwestern“ im Dialog. Frankfurt a. M.: Lang, S. 201-223.

Terhart, Ewald (2002): Fremde Schwestern. Zum Verhältnis von Allgemeiner Didaktik und empirischer Lehr-Lern-Forschung. In: Zeitschrift für Pädagogische Psychologie. Jg. 16. H. 2, S. 77-86.

Winkler, Iris: Wozu Literaturdidaktik? Perspektiven auf eine Disziplin zwischen den Stühlen. In: Doering, Sabine/Wätjen, Hans-Joachim (Hrsg.): Oldenburger Universitätsreden. Nr. 200. Oldenburg: BIS-Verlag, S. 9-31.

Winkler, Iris/Heinrich, Matthias/Fischer, Astrid/Krause, Ulrike (2016). Multiperspektivität in der Lehrerbildung. Fächerübergreifendes Lernen in der Mathematik- und Deutschdidaktik. In: Winkler, Iris/Schmidt, Frederike (Hrsg.): Interdisziplinäre Forschung in der Deutschdidaktik. „Fremde Schwestern“ im Dialog. Frankfurt a. M.: Lang, S. 181-199.

Zabka, Thomas (2012): Hinweise zum Aufbau literarischer Kompetenz in der Sekundarstufe II. In: Informationen zur Deutschdidaktik. Jg. 36. H. 1, S. 108-118. 
Iris Winkler and Frederike Schmidt - 978-3-631-69285-1

Downloaded from PubFactory at 01/11/2019 10:32:53AM

via free access 
Gerhard Rupp

\title{
Fremde und vertraute Schwestern - Konstellationen interdisziplinärer Forschung in der Deutschdidaktik seit den 90er Jahren ${ }^{1}$
}

\begin{abstract}
Interdisciplinary cooperation is the way by which „Deutschdidaktik“ has gained reputation in the academic world. This is especially true for the younger generation of „Deutschdidaktik“. Many teams have been formed by researchers of Educational Sciences, Psychometrics and Didactics of German Literature and Language. These teams and their findings are the starting point of further research and further successful cooperation - and indeed on an international scale.
\end{abstract}

\section{Fremde und vertraute Schwestern - Umrisse eines mittlerweile eingebürgerten Bildes}

Wenn man nach dem Stellenwert interdisziplinärer Forschung in der gegenwärtigen Deutschdidaktik fragt, kann ein Blick in die Geschichte der Disziplin klärende Funktion haben. Vor allem die Frage, wie sich das Verhältnis der Deutschdidaktik zu benachbarten Disziplinen entwickelt hat, rückt dabei in den Vordergrund.

Terhart (2002) geht von der Beziehung zwischen der Allgemeinen Didaktik und der Lehr-Lern-Forschung aus und zeigt auf, wie deren Annäherung letztlich fachdidaktische Perspektiven im interdisziplinären Dialog stärkt. Die Fremdheit der beiden ,Schwestern' Allgemeine Didaktik und Lehr-Lern-Forschung hat eine lange Geschichte, obwohl sie angesichts des geteilten Gegenstandsbereichs eigentlich paradox anmutet. Sie erklärt sich Terhart zufolge aber aus dem unterschiedlichen Selbstverständnis, der den beiden Schwestern eigen ist:

Lehr-Lern-Forschung ist - wie der Name schon sagt - ein Forschungsbereich innerhalb der Pädagogischen Psychologie. Die Allgemeine Didaktik ist demgegenüber gerade kein Forschungsbereich, sondern ein Element des Ausbildungsprozesses von angehenden Lehrern. Aufgrund dieses klaren Forschungsbezugs von Lehr-Lern-Forschung ist es auch angemessen zu sagen, dass sich die Forschungsstränge der Lehr-Lern-Forschung mit der theoriegeleiteten Beschreibung, Erklärung und Optimierung von Lehr-Lern-Prozessen befassen. (Terhart 2002, S. 80, Herv. ebd.)

1 Für wertvolle Hinweise zu diesem Beitrag danke ich Norbert Groeben. 
Allerdings überwinden beide Schwestern ihre Fremdheit. Terhart führt diesen Wandlungsprozess wissenschaftsintern auf die sog. kognitive Wende zurück. Beide Disziplinen konzentrieren sich nach dieser kognitiven Wende auf die Inhaltlichkeit des Lernens und tun dies mit Konzepten wie dem geordneten Wissensaufbau, der Problemlösefähigkeit, der Metakognition und der Selbstreflexivität. Besonders bedeutsam für die Deutschdidaktik ist eine der Perspektiven, die Terhart zum Schluss seiner Familiengeschichte ausweist. Es geht um die zunehmende Bedeutung von Fachdidaktiken, die zwar weiten Teils noch immer „systematisierte Handwerkslehren“ sind, aber zunehmend „fachspezifische Konkretisierungen allgemein-didaktischer Postulate [leisten können], die sich auf die Erfahrungsbasis von Praktikern in diesem Fach stützen" (Terhart 2002, S. 84). Innerhalb der Allgemeinen Didaktik kommt den verschiedenen Fachdidaktiken

die Aufgabe zu, die allgemeinen Reflexionen über Lehren und Lernen im Blick auf einen bestimmten Inhaltsbereich, im Blick auf ein bestimmtes Fach, im Blick auf einen bestimmten Lern- und Kompetenzbereich umzusetzen. (ebd.)

So kommt es laut Terhart zu einer „Höherbewertung der Fachdidaktik in der pädagogisch-didaktischen Diskussion“ (ebd., Herv. G. R.). Diese Höherbewertung ist gleichbedeutend mit der (endgültigen) Verwissenschaftlichung der Fachdidaktik und mit der interdisziplinären Position, die die Fachdidaktik zwischen Allgemeiner Didaktik und Lehr-Lern-Forschung einnimmt. Sie spiegelt sich in vielen Fällen in der Abkehr der Deutschdidaktik von der bisher meist betriebenen Einzelforschung und im produktiven Dialog mit verschwisterten Disziplinen u. a. im Kontext von Forschungsverbünden.

Einen anderen Akzent mit Blick auf die interdisziplinäre Stellung der Deutschdidaktik setzt Bettina Hurrelmann, wenn sie 1998, auf dem Siegener Symposion, aus der Sicht der Fachdidaktik „,Integrationsfelder' zwischen Fachwissenschaft und Fachdidaktik" (Hurrelmann 1998, S. 27, Herv. G. R.) ausmacht. Unter dem Titel „Deutschdidaktik - Kein Ort, nirgends?" hebt sie hervor, dass diese Felder weder zureichend aus der fachwissenschaftlichen noch allein aus der fachdidaktischen Perspektive heraus erforscht werden können. Hierzu zählen die Schriftlichkeitsforschung, die Erforschung des Erwerbs mündlicher Diskursfähigkeiten, die Kinder- und Jugendliteraturforschung, die Forschung zu Lesesozialisation, (neuen) Medien und der Multilingualität in einer multikulturellen Gesellschaft (vgl. Hurrelmann 1998, S. 27-30). All dies sind didaktisch und gesellschaftspolitisch hoch relevante Felder, die unter einem erheblichen Aktualitätsdruck bearbeitet und für das didaktische Handeln fruchtbar gemacht werden sollen. Hurrelmann macht fünf kennzeichnende Dimensionen aus, die die deutschdidaktische Forschung in diesen Integrationsfeldern auszeichnen: 
[Die] fachspezifische Gegenstandsentfaltung, [die] Medialitätsperspektive, [die] Erwerbsperspektive, [die] Empirieorientierung [und den] Anwendungsbezug. (Ebd., S. 31)

Für zwei dieser Integrationsfelder, für die Lesesozialisation und die Medien, ist diese Neuausrichtung der Deutschdidaktik mit der Bewilligung des sechsjährigen DFG-Schwerpunktprogramms "Lesesozialisation in der Mediengesellschaft" von 1998 bis 2004 unmittelbar darauf Wirklichkeit geworden. An den insgesamt ca. zwölf Projekten war die Fachdidaktik neben der Psychologie, der Soziologie, der Publizistik und der Medienwissenschaft etwa zu einem Viertel mit eigenen Projekten vertreten (vgl. Groeben/Hurrelmann 2004).

Mit Hurrelmann und Terhart lassen sich zwei Stimmen benennen, die um die Jahrtausendwende herum eine bedeutsame Zeitenwende für die Deutsch- bzw. für die Fachdidaktik allgemein konstatieren. Hurrelmann und Terhart konzentrieren sich im Wesentlichen auf wissenschaftsinterne Veränderungen, wobei allerdings Hurrelmann mit den aufgewiesenen Integrationsfeldern auf den soziokulturellen Wandel und auf die veränderten kulturellen Praktiken Jugendlicher abhebt.

Die wissenschaftsinternen Vorgänge konvergieren jedoch mit $u$. U. viel wirkungsmächtigeren wissenschaftsexternen Entwicklungen der Studienreform und der Bildungspolitik, die zu einer nachhaltigen sozialen Aufwertung der Fachdidaktik führen. In der neuen BA-MA-Studienstruktur werden zum ersten Mal in der Bildungsgeschichte fachdidaktische Lehrstühle gezielt mit Fachdidaktikern besetzt, die sich durch einschlägige Publikationen und in der Regel mit einer mehrjährigen Schulpraxis ausgewiesen haben. Und die nach wie vor schlechten Leistungsstände deutscher Schülerinnen und Schüler im internationalen Vergleich führen dazu, dass verstärkt Anstrengungen unternommen werden, die Kompetenz- und die Standardorientierung in Schule und Lehrerbildung an deutschen Hochschulen zu stärken (vgl. u. a. Bundesministerium für Bildung und Forschung 2013).

Als erstes Fazit meiner Einleitung lässt sich festhalten: Fach- und damit auch deutschdidaktische Forschung hat zur Jahrtausendwende eine bedeutsame Veränderung erfahren. Sie hat sich durch ihre Forschungsausrichtung nach den anfänglichen Versuchen seit Ende der 70er Jahre endgültig als Wissenschaft konstituiert, nicht nur durch Selbsterklärung, sondern durch die Anerkennung vertrauter Schwestern wie der Allgemeinen Didaktik und später auch fremderer Schwestern wie der Psychologie. Durch diese Entwicklungen hat sich die deutschdidaktische Forschung zumindest in Teilbereichen als Verbundforschung und als reviewte, anerkannte Forschung etabliert. Auslöser dieser Entwicklung sind u. a.

- der soziokulturelle Wandel,

- bildungspolitische Initiativen wie PISA als Druck auf die Ausbildungssysteme, 
- die durch PISA bewirkte Internationalisierung des Blicks auf Bildung, Erziehung und Unterricht sowie die Auswirkungen der Bologna-Studienreform auf die Lehrerbildung.

Mein Beitrag bringt im Folgenden:

- eine chronologische Rekonstruktion der Forschungsentwicklung seit den 90er Jahren und

- die systematische Bewertung der Forschungsentwicklung durch die Beantwortung der folgenden drei Fragen:

1. Was haben die einzelnen Forschungsverbünde für die Deutschdidaktik, aber auch für die beteiligten fremden Schwestern gebracht und was soll wieder auf die Tagesordnung gesetzt werden?

2. Wie ist der Prozess der Bildung von Forschungsverbünden seit den 90er Jahren zu bewerten?

3. Welche Forschungsverbünde werden in der Gegenwart gebildet und welche Forschungs-Optionen werden in der Zukunft mit welchen Zielen gewählt?

\section{Chronologische Rekonstruktion der Forschungsentwicklung seit den 90er Jahren}

Für die chronologische Rekonstruktion der Forschungsentwicklung seit den 90er Jahren benötigen wir ein Rahmenschema für die einzelnen Stufen oder Phasen, in die sich die Forschungsentwicklung untergliedert. Folgende Gesichtspunkte für diese Untergliederung sind ausschlaggebend: In der Einleitung hat sich die Jahrtausendwende bereits als bedeutsamer Einschnitt erwiesen, die man als Kernphase der empirischen Wende in der Deutschdidaktik bezeichnen kann, welche zur interdisziplinären Verbundforschung führt. Auch in diese Kernphase fallen die internationalen Large-Scale-Untersuchungen, bei denen wie bei PISA und IGLU Deutschdidaktiker als Berater, dann bei DESI als gleichberechtigte Forscher mitwirken. Dieser Kernphase deutschdidaktischer Forschung von 1998-2005 geht eine Vorbereitungsphase in den 70er, 80er und 90er Jahren voraus. Diese ist durch bedeutende Einzelforscher wie Jürgen Grzesik, Hubert Ivo oder Harro Müller-Michaels gekennzeichnet und durch einzelne empirische Projekte wie „Angenommen: Agamemnon“ von Ernst Nündel und Werner Schlotthaus. ${ }^{2}$ Der

2 Vgl. Grzesik (1976); Ivo (1977); Müller-Michaels/Rupp (1978); Nündel/Schlotthaus (1978). Für einen Überblick vgl. Becker-Mrotzek (1997); Müller-Michaels (1994) sowie Rupp/Bonholt (2006). 
Kernphase der empirischen Wende folgt 2005-2015 eine Differenzierungsphase. In dieser Phase schlägt zum einen das Pendel wieder stärker zur pädagogischpsychologischen Forschung aus. Man elaboriert das kompetenzorientierte Paradigma in einem weiteren DFG-Schwerpunktprogramm „Kompetenzdiagnostik“ unter verringerter Beteiligung der Fachdidaktik. Andererseits werden die seit DESI bestehenden Forschungsverbünde wieder neu aufgegriffen. So läuft mit ähnlich wie bei DESI gelagerter didaktischer Beteiligung und gefördert vom BMBF (Bundesministerium für Bildung und Forschung) von 2008-2012 die TEDS-LT-Studie (Teacher Education and Development Study: Learning to Teach). Schließlich gibt es in dieser Differenzierungsphase auch erstmals völlig in deutschdidaktischer Hand liegende eigenständige Forschung. Beispiel ist hierfür das durch Christine Garbe, Karl Holle und Swantje Weinhold geleitete internationale Leseförderungsprogramm „ADORE“von 2006-2009. ADORE nimmt damit die nächste, ab 2015 beginnende Autonomisierungsphase vorweg, in der Deutschdidaktikerinnen und Deutschdidaktiker eigenständig disziplinäre Forschungsprojekte initiieren. Diese Forschungsprojekte implizieren allerdings fast niemals die Rückkehr zur Einzelforschung der Vorbereitungsphase, sondern die Nutzung der Erkenntnisse der bisherigen Forschungserfahrung und ihrer Maximen zum Ausbau eigenständiger Perspektiven, zur Bearbeitung eigenständiger deutschdidaktischer Themen, zur Wahl angemessener affiner Untersuchungsverfahren und zur Zusammenstellung entsprechender Forschungsverbünde. Auffällig ist, dass nunmehr deutschdidaktische Kollegen pädagogische Psychologen ,mit ins Boot holen' und nicht umgekehrt.

In der Übersicht ergibt sich der folgende Ablauf, der nunmehr nochmals vertieft beleuchtet wird:

1. Vorbereitungsphase: 1945-1990: Wandel der Deutschdidaktik von der Methodik zu einer empirischen Wissenschaft

2. Kernphase: Empirische Wende 1990-2005

2.1. Internationale Large-Scale-Untersuchungen: PISA ( $\mathrm{ab}$ 2001), IGLU (ab 2003), DESI (2001-2007)

2.2. Interdisziplinäre Verbundforschung 1998-2005: z. B. Schwerpunktprogramm Lesesozialisation in der Mediengesellschaft

3. Differenzierungsphase, Nachfolge Verbundforschung 2005-2015: z. B. Schwerpunktprogramm Kompetenzdiagnostik, ADORE, TEDS-LT etc.

4. Autonomisierungsphase, eigenständige disziplinäre Forschung ab ca. 2015: z. B. Belke et al. 2015; Becker-Mrotzek et al. 2015; Dawidowski 2016; Pieper et al. 2012; Pieper 2014; Schneider et al. 2012. 


\subsection{Vorbereitungsphase 1945-1990}

Die hier aufgrund der Fokussierung auf die didaktische Wende so genannte Vorbereitungsphase ist die zeitlich am längsten währende Phase der Nachkriegszeit bis etwa 1990, dem Beginn der Kernphase der empirischen Wende. Sie untergliedert sich in drei voneinander abzusetzende Phasen, die man ihrerseits als Annäherung an die Empirie kennzeichnen kann: am Beispiel der Sprachdidaktik sind dies die Tradierung didaktischen Brauchtums ${ }^{3}$, die Umsetzungs- oder Anwendungsdidaktik und die empirische Sprachdidaktik. ${ }^{4}$

\subsubsection{Tradierung didaktischen Brauchtums (Methodiken): 1945-1970}

Zu Anfang reduziert sich die Didaktik auf Methodik und auf eine vorwissenschaftliche Handwerkslehre. Durch Lehrpläne und/oder fachwissenschaftliche Empfehlungen vorgegebene Unterrichtsinhalte wurden nicht in Frage gestellt, sondern selbstverständlich und unkritisch übernommen. In der Nachkriegszeit benötigte man neben der weitgehend verdrängten kritischen Aufarbeitung des Nationalsozialismus die Sicherheit vermittelnde Orientierung an unhinterfragten Inhalten, Gegenständen und überkommenen restaurativen Wertvorstellungen wie z. B. Ulshöfers Orientierung am „Leitbild eines ritterlichen (adeligen) Menschen“ (Ulshöfer 1957, S. 59). Literatur diente als Hilfestellung zur Lebensbewältigung, als unhinterfragtes ,Erlebnis', der Deutschunterricht insgesamt diente zur sprachlichen Bildung (vgl. Essen 1956) der Lernenden.

Auch didaktisch-methodische Vermittlungs- oder Korrekturverfahren wurden als ,Rezept-Wissen' von Lehrergeneration zu Lehrergeneration weitergegeben, ohne dass darüber nachgedacht wurde. Ein typisches Beispiel hierfür ist aus dem Bereich des Korrekturverhaltens das Anstreichen des sogenannten Wiederholungsfehlers, für das es keine sprachwissenschaftlich-sprachdidaktische Begründung gibt. Nach Sieber (1998) war

Voraussetzung für fachdidaktisches Arbeiten [...] in erster Linie erfolgreiche Unterrichtserfahrung und weniger die Kenntnis relevanter sprachwissenschaftlicher und didaktischer Zusammenhänge. (Sieber 1998, S. 357)

Die Fachwissenschaft war der Unterrichtspraxis als Instanz der Auswahl der Unterrichtsgegenstände vorgelagert, die Fachdidaktik verstand sich als nachgeordnete Methodik, die sich um das Wie der Umsetzung im Unterricht kümmerte.

3 Vgl. zu diesem auf Ivo (1977, S. 56) zurückgehenden Begriff die Wiederaufnahmen bei Ossner (1993, S. 194ff.); Becker-Mrotzek (1997, S. 17) und Sieber (1998, S. 357).

4 Vgl. Becker-Mrotzek (1997); Sieber (2005). 
Allenfalls kam es zur Reflexion der Praxis in den Beiträgen der von Ulshöfer begründeten und herausgegebenen Zeitschrift „Der Deutschunterricht“ ab 1948.

\subsubsection{Umsetzungsdidaktik, Anwendungsdidaktik: 1970-1980}

Umsetzungs- und Anwendungsdidaktik stellen zum ersten Mal die didaktische Frage nach der Auswahl der Unterrichtsgegenstände, z. B. durch den Bezug auf die Sprachwissenschaft und die Relevanz der jeweiligen Theorie-Ansätze. ${ }^{5}$ So werden Paradigmen der Sprachwissenschaft wie die Transformationsgrammatik im Zuge der Linguistisierung des Deutschunterrichts unmittelbar in Konzepten für den Unterricht umgesetzt.

Die Anwendungsdidaktik bezieht sich ebenfalls auf Paradigmen der Sprachwissenschaft wie die Soziolinguistik und will damit z. B. zur Sprachförderung durch die Aufhebung sozial bedingter Sprachbarrieren beitragen. Durch den Bezug auf die Pragmatik fördert sie das Ziel mündlicher Kommunikationsfähigkeit, z. B. indem Arbeitsformen wie das Rollenspiel empfohlen werden.

Umsetzung- und Anwendungsdidaktik markieren mit der Hervorhebung und mit der Betonung der Didaktik den Wissenschaftsbezug der Deutschdidaktik. Allerdings stehen dabei immer noch aus den Fachwissenschaften übernommene Fragestellungen im Mittelpunkt und noch keine eigenen Fragestellungen oder Paradigmen. Entsprechend geschieht die Umsetzung und/oder Anwendung vorschnell und kurzschrittig und verhilft der Deutschdidaktik vorerst nicht zur Emanzipation als eigenständige Disziplin.

\subsubsection{Erfahrungswissenschaftliche Didaktik: 1980-1990}

Durch die erfahrungswissenschaftliche bzw. empirische Sprachdidaktik werden zum ersten Mal in der Geschichte der Deutschdidaktik die Wirkungen didaktischen Handelns durch die systematische Beobachtung, Dokumentation und Reflexion von Lehr-Lernprozessen überprüft:

Bevor Praxis verändert werden konnte, musste untersucht werden, wie Unterricht überhaupt läuft, wie Schüler denken und handeln und welche Interessen sie verfolgen. Handlungsorientierung und empirische Studien haben insofern miteinander zu tun, als sie beide bei der Kategorie Schüler ansetzen, deren Entwicklungen, Neigungen und Erfahrungshorizonte studieren, um sie produktiv zu beeinflussen. (Müller-Michaels 1994, S. 40)

5 Mit Umsetzungsdidaktik ist die Nutzung oder die Thematisierung fachwissenschaftlicher Theorien gemeint. Mit der Anwendungsdidaktik werden darüber hinausgehend methodische Konzepte bezeichnet, die aus den fachwissenschaftlichen Theorien folgen. 
Während das Studium der „Entwicklungen, Neigungen und Erfahrungshorizonte" der Schüler kaum regelgeleitet betrieben wird, kommen durch die Empirie schrittweise Kriterien strengerer Wissenschaftlichkeit ins Spiel. Nach BeckerMrotzek tritt an „die Stelle der theoretischen Deduktion aufgrund von Erfahrung [...] die empirische Rekonstruktion authentischer Wirklichkeitsbeobachtung" (Becker-Mrotzek 1997, S. 21). Damit ist die seit 1977 angestrebte Wissenschaftlichkeit der Deutschdidaktik (Ivo 1977) vollendet: und zwar durch den Theoriebezug, durch die methodengeleitete „empirische Rekonstruktion authentischer Wirklichkeitsbeobachtung" (Becker-Mrotzek 1997, S. 21) mit angemessenen Verfahren und durch die Beachtung strenger wissenschaftlicher Regeln. Unter diesen Voraussetzungen können Lehr-Lern-Prozesse untersucht oder bestimmte Diskurstypen entdeckt werden wie z. B. der sog. Unterstützungsdiskurs in der Eltern-Kind-Kommunikation (vgl. ebd., S. 26).

Die der empirischen Wende vorauslaufende Phase zeigt, wie sich die Deutschdidaktik in einem relativ kurzen Zeitraum fundamental ändert und sich von einer Handwerkslehre zu einer empirischen Disziplin weiterentwickelt. Dies ist mit einem halben Disziplinwechsel vom hermeneutischen zum szientistischen Paradigma verbunden. Im Zeitraum von nur zehn Jahren vollziehen sich demnach bedeutsame Wandlungsprozesse.

\subsection{Kernphase Empirische Wende: 1990-2005}

Durch die zuletzt beschriebene Hinwendung der Deutschdidaktik zur Empirie ist die Disziplin einigermaßen vorbereitet auf den Umschwung, der sich um die Jahrtausendwende vollzieht, und der durch mehrere gleichzeitige oder kurz aufeinander folgende Entwicklungen gekennzeichnet ist:

1. Das Bildungssystem wird von der Input- auf die Outputorientierung umgestellt. Dies ist die Folge und zugleich das Ziel internationaler Large-ScaleUntersuchungen.

Bei den großen internationalen als Auftragsforschung der OECD durchgeführten Schulleistungsstudien PISA und IGLU wirken Deutschdidaktiker, wie z. B. Bettina Hurrelmann und Heiner Willenberg, beratend in deutlich nachgeordneter Position mit. D. h., dass Felder wie Lesen und Schreiben wie übrigens seit ehedem, also auch in den 80er und 90er Jahren und auch weitgehend heutzutage, fachfremd beforscht wurden. Dies ändert sich etwas bei der stärker fachdidaktisch ausgerichteten DESI-Studie von 2001 bis 2007, an der zumindest Deutsch- und Englischdidaktiker mitwirkten und sowohl domänenspezifische Kompetenz- wie psychometrische Messmodelle entwarfen. 
2. Im Bereich der Bildungsforschung werden umfangreiche Schwerpunktprogramme unter Einschluss der Fachdidaktik gegründet. Diese Großforschung und die Drittmitteleinwerbung gewinnen im Zuge von Zielvereinbarungen zwischen Hochschulleitungen und Professoren mehr und mehr an Gewicht. Durch den Nachweis von Forschungsleistungen kann die Deutschdidaktik das Reputationsgefälle zu den Fachwissenschaften und zu anderen Domänen, vornehmlich zu den MINT-Fächern, wenigstens ansatzweise ausgleichen.

3. Die Lehrerbildung wird in vielen Bundesländern auf die BA-MA-Struktur umgestellt. In den im Kontext dieser Struktur neben den Master of Arts-Studiengängen eingerichteten fachdidaktischen Master of Education-Studiengängen unterrichten u. a. grundständig ausgebildete Fachdidaktiker als Professoren. Dies führt zu einem deutlichen Stellenausbau der Fachdidaktiken; durch die Zentren für Lehrerbildung oder Professional Schools bekommen sie vielerorts einen ,Ort in der Hochschule (vgl. aber Böttcher/Blasberg 2015).

4. Innerhalb der Deutschdidaktik verläuft diese Entwicklung in der Sprach- und der Literaturdidaktik höchst unterschiedlich. Während in der Sprachdidaktik empirisch exakte und auch quantitative Methoden bereits seit einiger Zeit genutzt und entsprechende Arbeiten auch rezipiert werden, ${ }^{6}$ ist das in der Literaturdidaktik allenfalls in Ansätzen erkennbar. Beispielsweise werden die „empirischen Arbeiten von Jutta Wermke aus dem Jahre 1989 in der Literaturdidaktik so gut wie gar nicht rezipiert" (Frederking 2014, S. 116). Wermke hatte Kreativitätsförderung im Literaturunterricht mit quantitativen Methoden untersucht und evaluiert (vgl. Wermke 1994, S. 584).

\subsubsection{Umstellung von der Input auf die Output-Orientierung: Internationale Large-Scale-Untersuchungen}

Die durch die OECD initiierten internationalen Large-Scale-Untersuchungen lösten durch ihren Untersuchungsstil, durch die Durchsetzung der Psychometrie als verbindliche Messmethode für empirische Bildungsforschung und durch ihre meist skandalös schlechten empirischen Ergebnisse einen noch immer anhaltenden bildungspolitischen Schock aus. Sie haben die Forschungslandschaft und ansatzweise die Schul- und die Unterrichtswirklichkeit nachhaltig verändert.

Der Untersuchungsstil zeichnet sich durch den internationalen Vergleich, durch große Probandengruppen und durch zyklische Wiederholungen innerhalb eines

6 Dies gilt für die „Erforschung von Unterrichtshandlungen wie vor allem der Unterrichtskommunikation und des Schriftspracherwerbs“, wie Dawidowski (2009, S. 29) mit Blick auf die Beiträge in Kammler/Knapp (2002) zusammenfasst. 
jahrzehntelangen Untersuchungszeitraums aus. Durch diese zyklischen Wiederholungen von Messzeitpunkten mit unterschiedlichem thematischem Schwerpunkt wird die Nachhaltigkeit der Überprüfung und der bildungspolitischen Verbesserungen angezielt.

Forschungsmethodisch haben PISA und IGLU die Standards der Lehr-LernForschung auch für die fachdidaktische Forschung implizit verbindlich gemacht. Dies hat zur Konsequenz, dass theoretische Konzepte durch Testfragen operationalisiert und in psychometrische Messmodelle überführt werden sollen. Dies betrifft die Datenerhebung und die meist nach der Raschskalierung vorgenommene statistische Datenauswertung. Die hier zu leistende Übersetzung bzw. Reduktion anspruchsvoller theoretischer Modelle führt zu einer Überprüfung und kritischen Sichtung von Theorieannahmen, bei der sich die fremden Schwestern einander annähern oder so fremd bleiben, wie es die Reaktion eines nennenswerten Anteils der Deutschdidaktik zeigt (vgl. Abraham et al. 2003).

Während PISA und IGLU allgemeine Kompetenzmessungen vorlegen, die sie nachträglich theoretisch modellieren, und nur wenig Hinweise auf konkrete Verbesserungsmöglichkeiten der Unterrichtsqualität bieten, entwickelt DESI z. B. zum Lesen eine eigenständige theoretische Modellierung, die bereits Hinweise auf konkrete Fördermöglichkeiten der Lesekompetenz gibt. Dies geschieht beispielsweise durch die errechneten relevanten Korrelationen des Teilbereichs Lesen mit den anderen fünf Bereichen des Faches Deutsch:

Abb. 1: Korrelationen des Teilbereichs Lesen (Willenberg 2014, S. 559)

\begin{tabular}{|l|l|l|l|l|l|}
\hline TEILGEBIETE & Wortschatz & Argumentation & Sprachbewusstheit & Textproduktion & Rechtschreibung \\
\hline Lesen & .59 & .61 & .61 & .46 & .28 \\
\hline
\end{tabular}

Argumentation und Sprachbewusstheit sowie der Wortschatz weisen hohe signifikante Werte auf, während Textproduktion und Rechtschreibung wenig signifikante Werte zeigen. Durch Förderung der für das Lesen relevanten Teilbereiche des Deutschunterrichts kann, so die Interpretation der DESI-Forscher, also auch die Lesekompetenz erhöht werden.

\subsubsection{Großforschung: Interdisziplinäre Verbundforschung 2000-2005}

Beispielhaft für eine interdisziplinäre Verbundforschung steht das Schwerpunktprogramm "Lesesozialisation in der Mediengesellschaft“, das von 1998 bis 2005 gefördert wurde und seine inhaltlichen Ergebnisse u. a. in dem Band „Lesesozialisation in der Mediengesellschaft" und seine methodologischen Ergebnisse u. a. in dem Band „Empirische Unterrichtsforschung - ein Weiterbildungsprogramm“ 
zusammengefasst hat (vgl. Groeben/Hurrelmann 2004, 2006). Die hier realisierten deutschdidaktischen Projekte stechen durch ihre thematische Breite, durch die Vielfalt der Ansätze und durch die differenzierten Ergebnisse hervor: Themen sind Lesen und Medien sowie die Lese- und die Mediensozialisation in historischer und systematischer Hinsicht. Zahlreiche Verfahren der Datenerhebung und der Datenauswertung wurden eingesetzt: Biographiestudien, Feldanalysen, Leitfadeninterviews, Befragungen, kontrastive Tests in Haupt- und Vergleichsgruppen sowie Triangulationen. Heute noch bedeutsame Ergebnisse resultieren z. B. aus der Hauptschulstudie "Lesesozialisation in schriftfernen Lebenswelten“ von Pieper et al. 2004, aus der historisch ausgerichteten Gegenüberstellung der Lesesozialisationsbedingungen 1800, 1900 und 2000 im Projekt Hurrelmann, aus der Ermittlung von Buch- bzw. Medien-Sozialisationstypen in den Projekten Garbe und Rupp sowie aus der Überprüfung von Wirkungen des Literaturunterrichts im Projekt Fritzsche (Pieper et al. 2004; Hurrelmann/Groeben 2006; Schulte-Berge et al. 2002; Rupp et al. 2004; Fritzsche et al. 2006).

Wichtiger noch als diese Erträge deutschdidaktischer Forschung ist der Gewinn, den alle Beteiligten aus dem Miteinander mit den fremden und allmählich immer vertrauteren Schwestern und Brüdern gewonnen haben. Implizit haben alle Beteiligten gelernt, dass gemeinsame Forschung auf gemeinsam geteilten Begriffen beruht, ferner auf gemeinsam geteilten Methoden und Verfahren der Datenerhebung und der Datenauswertung. Hieraus rühren die Identität und die Wiedererkennbarkeit der Disziplin. Nicht ohne Grund waren die ersten Projektpublikationen grundlegenden Begriffen und grundlegenden Forschungsverfahren gewidmet (vgl. u. a. Groeben 1999). Schließlich hat das wissenschaftliche Denken eine spezifische Ablaufform, die allmählich immer mehr von deutschdidaktischen Kollegen übernommen wurde und die mit den Stationen Vorstrukturierung (Problembenennung) - Hypothesenbildung - Experiment (Durchführung) - Ergebnisdarstellung und Diskussion den zielführenden Weg der Erkenntnisgewinnung aufzeigt. Eine solche Matrix gilt und galt auch für zahlreiche Aufsatzformen. Diese Strenge des wissenschaftlichen Denkens und Schreibens in empirischen Forschungskontexten hatte sich auch an der schwierigen Textsorte des DFGAntrags zu bewähren.

Die interdisziplinäre Konstellation beförderte zweifellos sehr stark einen Braindrain von der Psychologie zur Didaktik. Umgekehrt stellten die Fachdidaktiken ihr reichhaltiges soziales Feld zur Verfügung: die spannenden Lernprozesse und -produkte sowie die einzelnen Phasen der Lese- und der Mediensozialisation, die in der Biographieforschung erhoben und ausgewertet werden. Zusätzlich komplettierten die Fachdidaktiken den theoretischen Rahmen z. B. durch die Auslotung 
des "geschichtlichen und kulturellen Umfeld[s]“ (Rupp/Bonholt 2006, S. 53) und die Explikation „lesedidaktische[r] Konzepte“ (Nickel-Bacon 2006, S. 95). Durch die somit vollzogene Integration in die sozialwissenschaftliche Empirie hat die Deutschdidaktik somit die Anerkennung als Forschungsdisziplin und damit innerhalb der Fächergemeinschaft ein zunehmend selbstbewusstes Standing gewonnen, das sie in ihrem Bestand sichert und festigt.

Bei all diesen positiven Aspekten dürfen die schwierigen Begleiterscheinungen dieser Entwicklung nicht verschwiegen werden. So mussten sich diejenigen Deutschdidaktiker, die von der skizzierten Entwicklung profitieren wollten, innerhalb kürzester Zeit in Verfahren, Normen und Kriterien der meist exakt arbeitenden Lehr-Lern-Forschung einarbeiten bzw. diese für das eigene Arbeiten und die eigenen Fragestellungen übernehmen. Dies bedeutete sehr oft, ,weiche' Fragestellungen disziplin-spezifischer Natur aufzugeben bzw. hintan zu stellen. Das Prestige und auch die Corporate Identity von Psychologie, Erziehungswissenschaft und Lehr-Lern-Forschung waren aber auch nach vollzogener Anpassungsleistung seitens der Deutschdidaktik so stark, dass die Verbünde bis auf wenige Ausnahmen von Vertretern der anderen Disziplinen angeführt wurden und Deutschdidaktiker sich mit der Rolle als nachgeordnete Partner zufrieden gaben. Dies erforderte starkes Selbstvertrauen bzw. Durchhaltevermögen bis zum gegenwärtigen Zeitpunkt der 10er Jahre des 21. Jahrhunderts, in denen sich eine Rückbesinnung auf die Stärken der eigenen Disziplin der Deutschdidaktik immer mehr abzeichnet.

Zurück zu den positiven Aspekten der Kooperation und hier den persönlichen Aspekten, die nicht unerwähnt bleiben dürfen. Dies betrifft zunächst die solidarische Kooperation, die durch die Kollegen z. B. aus der Kulturpsychologie den deutschdidaktischen Kollegen entgegengebracht wurde. Diese Kooperation wirkte sich durch die Beratung, die Anregung sowie durch unermüdliche Hilfestellungen bei den Antragsstellungen aus.

Weiterhin ist der personelle Austausch zwischen den Disziplinen bemerkenswert. Mehrere Kollegen wechselten die Seiten zwischen psychologischen und literaturdidaktischen Projekten. Schließlich ist die nachdrückliche Nachwuchsförderung zu erwähnen, durch die zahlreiche junge Kollegen Lebenszeitstellen und Professuren erringen konnten. Dies entsprach dem Ziel des Schwerpunktprogramms, die intendierten Forschungsüberzeugungen durch die Berufung junger Wissenschaftler in Lebenszeitprofessuren zu realisieren, was für zahlreiche Forscher aus beiden Disziplinen zutrifft. Dass das zweite Ziel, die institutionelle Durchsetzung des Forschungs-Weiterbildungsprogramms, z. Zt. nicht institutionalisiert ist, ist demgegenüber nachrangig (vgl. Hurrelmann/Groeben 2006; Groeben 2005, bes. S. 28-30). 


\subsection{Differenzierungsphase: $2005-2015$}

In der Differenzierungsphase von 2005-2015 werden die Aspekte der interdisziplinären Konstellation zwischen der Unterrichtsforschung und den Fachdidaktiken weiter präzisiert. In der Programmatik des Schwerpunktprogramms „Kompetenzdiagnostik“ werden erstmals die „kognitionspsychologischen und fachdidaktischen Grundlagen von Kompetenzen“ und die „psychometrische(n) Modelle und konkrete(n) Technologien für ihre Messung" einander gleichberechtigt gegenüber gestellt. Thematisch geht es in der Förderphase von 2007-2013 u. a. um schulische und berufliche Bildung, mehrfache Raschmodelle und um dynamisches adaptives Testen. ${ }^{7}$ Einziges Projekt mit deutschdidaktischer Beteiligung ist die Erforschung der literarästhetischen Urteilskompetenz (vgl. Frederking et al. 2008; Frederking/ Henschel, in diesem Band). Die literarästhetische Urteilskompetenz wird in die drei Dimensionen semantisches literarästhetisches Urteilen, idiolektales literarästhetisches Urteilen und kontextuelles literarästhetisches Urteilen aufgeteilt. Diese Dimensionen sind in der ersten Projektphase unter Berücksichtigung von Annahmen über Anforderungsniveaus mit Aufgaben operationalisiert und erprobt worden.

Daneben wird in der Differenzierungsphase die Kooperation zwischen Pädagogischer Psychologie und Deutschdidaktik fortgeführt. Hierfür steht u. a. das erfolgreiche Frankfurter Leseflüssigkeits-Projekt von Gold und Rosebrock (2006-2008). Leseflüssigkeit bezieht sich auf die Leseprozesse im hierarchieniedrigen Bereich. ${ }^{8}$ Man versteht darunter eine grundständige Voraussetzung für Leseverstehen, die aus der genauen und automatisierten Dekodierfähigkeit, der angemessenen Lesegeschwindigkeit und der „Fähigkeit zum ausdrucksstarken Vorlesen“ besteht (Rosebrock/Nix 2008, S. 38):

Im Projekt werden die Effekte zweier unterrichtlicher Settings zur Förderung von Fluency auf die Lesegeschwindigkeit, die Textverstehensleistung und die Lesemotivation in insgesamt 24 Hauptschulklassen der 6. Jahrgangsstufe im Einzugsbereich Frankfurts bestimmt: Jeweils acht Klassen erfahren ein Treatment mit einem „Sustained Silent Reading“-Programm bzw. mit einem „Oral Reading"-Setting über jeweils ein halbes Schuljahr $(3 \times 20$ Min/Woche), acht weitere Klassen bleiben als Kontrollgruppe. ${ }^{9}$

7 Vgl. http://kompetenzmodelle.dipf.de/pdf/Kompetenzmodelle_Flyer_d_27032012_ rz.pdf (abgerufen am 06.05.2015).

8 „Der Erwerb von Leseflüssigkeit ist [...] nach der alphabetischen Phase und vor der Fähigkeit zum effizienten Umgang mit längeren Texten angesiedelt" (Rosebrock/Nix 2008, S. 35).

9 Vgl. http://www.uni-frankfurt.de/42717567/Lesefluessigkeit (abgerufen am 06.05.2015). Vgl. zusammenfassend Rosebrock et al. (2011). 
Eine erste, wenn nicht die erste internationale Forschungskooperation wurde durch das europäische Forschungsprogramm des Referenzrahmens Literatur LiFT-2 („Literary Framework For Teachers in Secondary Education“) gebildet:

Innerhalb des Projektes LiFT-2 wurde ein Referenzrahmen für die Altersgruppen der 12bis 19-Jährigen entwickelt, der Anforderungsniveaus unterscheidet, diesen Niveaus Bücher zuordnet und Vorschläge für didaktische Übergänge macht. Das Projekt wurde von 2009 bis 2012 im Rahmen des EU-Comenius-Programmes durchgeführt. Teilnehmende Länder waren die Niederlande (Federführung), Tschechien, Finnland, Deutschland, Rumänien und Portugal. Auf der Grundlage von Expertendiskussionen mit Lehrkräften aller teilnehmenden Länder wurden Leseniveaus von Schülerinnen und Schülern beschrieben. Dies diente - neben einem Vergleich der Kerncurricula aller teilnehmenden Länder - der Erschaffung eines europäischen Referenzrahmens für Literatur. (Pieper et al. 2012, S. 5)

Bei LiFT-2 (vgl. auch Pieper/Sâmihăian, in diesem Band) unterscheidet man das eigentliche vorausgegangene interdisziplinäre Forschungsprojekt, das zur Erstellung des literarischen Referenzrahmens geführt hat, und die Nutzung und Erprobung des Referenzrahmens durch die Lehrkräfte in den angesprochenen europäischen Ländern. Die Antworten auf Forschungsfragen wie die der literarischen Kompetenzentwicklung, der literarischen Entwicklung und Progression sowie der dazu passenden literarischen Texte wurden nicht nur national, sondern kulturvergleichend in internationalem Maßstab beantwortet. Forschungsmethodisch wurden Expertendiskussionen unter den beteiligten Lehrkräften genutzt, um ihr „professionelles Wissen im Sinne des Pedagogical Content Knowledge (Shulman 1986) zu rekonstruieren (vgl. Pieper 2014, S. 588). Die zu dem Projekt gehörende Webseite ermöglicht den gleichfalls internationalen Austausch über die Projektergebnisse.

Als Beispiel für die Kooperation zwischen Unterrichtswissenschaft und Deutschdidaktik und zwischen zwei benachbarten Hochschulstandorten steht das Bochum-Wuppertaler Kooperationsprojekt „Textkohäsion als Bedingung des Leseverständnisses“ (Rupp/Gräsel) von 2012-2014. Rupp und Gräsel untersuchten den Einfluss der lokalen vs. globalen Kohäsion von Sach- und literarischen Texten auf das Textverständnis und variierten in einem 2x3-Design den Inhalt, den Texttyp und die Lesererwartung. Als Ergebnis resultierte die geringe Signifikanz der in den Testtexten vorgenommenen Manipulationen, durch die jeweils eine wenig bzw. besonders ausgeprägte lokale und globale Kohäsion erreicht wurde. Im Folgeprojekt 2013-2015 wurde deshalb das auf nominalen Markern basierende Kohäsionskonzept um strukturelle und diskurstheoretische Kategorien wie das Tempus erweitert (vgl. Rothstein et al. 2014). 
Als Teilfortführung der DESI-Studie lässt sich das BMBF-Projekt TEDS-LT 2008-2012 verstehen, das unter der Beteiligung deutschdidaktischer Kollegen durchgeführt wurde.

TEDS-LT zielt im Rahmen einer Längsschnittuntersuchung auf die Beschreibung des professionellen Wissenserwerbs und der Lerngelegenheiten von Lehramtsstudierenden im Bachelor- und Masterstudium bzw. in den grundständigen Staatsexamensstudiengängen. Die Zielpopulation von TEDS-LT besteht aus Lehramtsstudierenden der Unterrichtsfächer Deutsch, Englisch und Mathematik, die die Lehrbefähigung für die Sekundarstufe I anstreben. (Buchholtz et al. 2011, S. 25)

Die für TEDS-LT vorgegebene Testzeit ließ

nur die Möglichkeit, sich für die Untersuchung einzelner Dimensionen zu entscheiden. Positiv gesehen ermöglicht es die Begrenzung aber, das fachwissenschaftliche Wissen mit jeweils zwei Subdimensionen abzubilden, und zwar für die Sprachen differenziert in Literatur- und Sprachwissenschaft sowie für Mathematik in Arithmetik und Algebra. (Blömeke 2011, S. 14)

Im Fach Deutsch wurden fünf linguistische Subdimensionen von Phonetik bis Pragmatik und fünf literaturwissenschaftliche Subdimensionen von „Autoren, Werke[n] bzw. Medien vornehmlich des 20. Jahrhunderts und der Gegenwart“ bis zu den Methoden der Textanalyse/Textinterpretation verwendet (vgl. Bremerich-Vos et al. 2011, S. 50f.). Eines der Hauptergebnisse der empirischen Testung besteht in dem Mangel an systematischer Vermittlung von Überblickswissen:

Viele Studierende wiesen nach der Testung darauf hin, dass sie sich zwar mehr oder weniger dunkel an den ,Stoff‘ erinnern könnten. Er sei in der Lehre in der Folge aber nicht mehr systematisch aufgegriffen worden. Daraus mag man schließen, dass es jedenfalls aus der Sicht der Studierenden keine auf kumulatives Lernen hin angelegte Lehre gibt. Kompetenzorientierte Lehre sollte aber vor allem darin bestehen, dass den Lernenden immer wieder Gelegenheit gegeben wird, in multiplen Kontexten intelligent zu üben, so dass es zu den gewünschten Transfereffekten kommen kann. (ebd., S. 72)

Vollends unter deutschdidaktischer Ägide lief von 2007-2009 das Projekt "ADORE - Teaching Struggling Adolescent Readers in European Countries“ als EU-Projekt. In elf europäischen Ländern wurde durch Lehrplansichtung, Unterrichtsbeobachtung und Leitfadeninterviews mit Schüler und Lehrer die jeweilige Leseförderpraxis per Erziehungssystemvergleich erhoben. Die Studie folgt einem qualitativ-heuristischen Konzept und zielt auf die bildungspolitische Veränderung und Vernetzung der Leseförderpraxis in den einzelnen europäischen Ländern. Als „superior goal“ listet sie „Changing the self-concept of ASR [i.e. Adolescent Struggling Readers]“ (Garbe et al. 2009, S. 6) sowie „6 key elements of good practice“ (ebd., S. 75-222) auf, die in einem weit gespannten Bogen alle 
Ebenen des Leseprozesses, der Leseförderung und der institutionellen Bedingungen umfassen. Die quantitative Überprüfung bestimmter Treatmenteffekte tritt dabei zurück. Ein problematischer Punkt des ADORE-Projekts besteht u. a. in der implizit normativ vollzogenen Setzung, „als ,beste Praxis für Lese(kompetenz-) förderung generalisierend eine Praxis zu definieren, die sich in allen Fächern manifestiert" (Scherf 2013, S. 425). Trotzdem gibt ADORE in seiner Formation als eigenständiges internationales Forschungsprogramm die Blaupause für die letzte Autonomisierungsphase ab 2015 ab.

\subsection{Autonomisierungsphase ab 2015}

Wie schon ADORE zeichnen sich die zahlreichen gegenwärtigen Projekte durch eigenständige Initiierungen von Seiten der Deutschdidaktik aus. Sie sind thematisch breit gestreut, durch unterschiedliche Förderinstitutionen unterstützt und in unterschiedlichen „Formaten fachdidaktischer Forschung" realisiert (vgl. Bayrhuber 2012). Dabei ist es nur möglich, einige Schlaglichter auf die gegenwärtige Entwicklung zu werfen, zu der man als Beobachter nur einen eingeschränkten Zugang hat.

Systematisch lassen sich drei Formate voneinander abgrenzen: internationale, interdisziplinäre und deutschdidaktische Forschungsprojekte.

\subsubsection{Internationale Projekte}

Das Ende 2014 bewilligte Bochum-Genfer Forschungsprojekt „Psycholinguistic perspectives on orthography instruction and literacy acquisition" kann als Flaggschiff der ersten Kategorie gelten. Es wurde von den Bochumer Sprachwissenschaftlerinnen Eva Belke und Stefanie Dipper, der Genfer Neurobiologin Sonia Kandel und der Bochumer Sprachdidaktikerin Claudia Müller im VW-Programm „Key Issues for Research \& Society“ unter dem Titel „Literacy as a key to social participation" beantragt.

Dieses Projekt ist insofern exemplarisch, als es in einem außergewöhnlich kompetitiven Wettbewerb den Zuschlag und als einzelnes Verbundprojekt die Fördersumme von fast 1 Mio $€$ erteilt bekommen hat. Weiterhin ist die interdisziplinär-internationale Zusammensetzung der Forschergruppe insbesondere mit der Genfer Neurobiologin Sonia Kandel bemerkenswert. Obwohl mit Claudia Müller nur eine Deutschdidaktikerin in diesem Projekt vertreten ist, ist die Leithypothese des Projekts fachdidaktischer Natur und zielt auf die sprachstatistisch absehbare Wirkkraft impliziten Lernens sowie auf die daraus abzuleitenden Konsequenzen, die eine eigens hierfür geschaffene Professur ableiten soll: 
Implicit learning enables speakers to acquire knowledge of the statistical properties of written utterances, i. e. patterns of co-occurrence and contingencies of letters in written word forms arising from graphotactic, phonological and morphosyntactic principles in orthography. Critically, unlike the standard belief, implicit learning requires that the linguistic input be optimized for it to occur. [...] In corpus linguistic and experimental studies as well as an intervention study, we will assess the predictions following from this hypothesis. A research professorship flankering these research strands is aimed at compiling psycholinguistic, linguistic, didactic and cognitive psychological evidence in order to put forward a working model of language and literacy acquisition in preschool and primary school children. ${ }^{10}$

\subsubsection{Interdisziplinäre Forschungsprojekte}

Als interdisziplinäres Großprojekt darf das Entwicklungs- und Forschungsprogramm „Bildung durch Sprache und Schrift (BiSS)“ gelten. Es wurde am 1. September 2013 mit einer Laufzeit von fünf Jahren gestartet. BiSS ist eine gemeinsame Initiative des Bundesministeriums für Bildung und Forschung (BMBF), des Bundesministeriums für Familie, Senioren, Frauen und Jugend (BMFSFJ) sowie der Kultusministerkonferenz (KMK) und der Konferenz der Jugend- und Familienminister (JFMK) der Länder zur Verbesserung der Sprachförderung, Sprachdiagnostik und Leseförderung. Das Mercator-Institut für Sprachförderung und Deutsch als Zweitsprache der Universität zu Köln, das Deutsche Institut für Internationale Pädagogische Forschung (DIPF) und die Humboldt-Universität zu Berlin in Kooperation mit dem Institut zur Qualitätsentwicklung im Bildungswesen (IQB) übernehmen als Trägerkonsortium die wissenschaftliche Ausgestaltung und Gesamtkoordination des Programms. Kindergärten, Kindertagesstätten und Schulen haben sich mit einzelnen Förderkonzepten beworben.

Dieses Projekt belegt, dass die Deutschdidaktik mittlerweile eine gleichberechtigte leitende Position in national angelegten Großforschungsprojekten einnimmt. Dies war vor gut zehn Jahren noch nicht der Fall. Außerdem handelt es sich um eine Art Selbstreflexion der Deutschdidaktik, insofern als die Wirksamkeit derjenigen Förderkonzepte unter die Lupe genommen wird, die zwar mittlerweile gut bekannt, aber doch erst seit ca. 15 bis 20 Jahren diskutiert werden.

BiSS zielt mit einzelnen thematischen Modulen auf den Elementar-, den Primar- und den Sekundarbereich. Zentrale Handlungsfelder sind die Sprachförderung insbes. des Deutschen als Zweitsprache, die Sprachdiagnostik, die Lese- und die Schreibförderung. Die thematische Bandbreite reicht von der Förderung

10 Vgl. http://staff.germanistik.rub.de/claudia-mueller/wp-content/uploads/Projektzusammenfassung.pdf (abgerufen am 30.06.2015). 
phonologischer Bewusstheit als Vorläuferfähigkeit der Lesekompetenz über das dialogische Lesen als Form kooperativen Lernens bis zur Diagnose und Förderung der Leseflüssigkeit und der Vermittlung von Lese- und Schreibstrategien mit digitalen Medien (vgl. Schneider et al. 2012, S. 22; kritisch dazu: Ehlich/ Valtin 2012). Mit diesen Thematiken werden zugleich Schwerpunkte der Forschungskooperationen der vorausgegangenen Jahre wieder aufgenommen und jetzt breit und vertieft zahlreichen Praxistests unterzogen, deren Evaluation z. Z. noch aussteht:

Ziel von BiSS ist es also, Erkenntnisse darüber zu gewinnen, welche Maßnahmen der Sprach- und Leseförderung in den verschiedenen Etappen des institutionellen Bildungsweges - vom Elementarbereich über die Grundschule bis zur Sekundarstufe - sich in der pädagogischen Praxis bewähren und Wirkungen zeigen. Auf Wunsch der Initiatoren sollten dabei Ansätze der Förderung aufgegriffen, gebündelt und evaluiert werden, die bereits umgesetzt werden. Um eine Bündelung solcher Maßnahmen zu ermöglichen, wurden in einem ersten Schritt in einer Expertise (Schneider et al. 2012) sog. Module definiert, die für alle Bildungsetappen und für unterschiedliche Bereiche Maßnahmen beschreiben, deren Wirksamkeit entweder empirisch belegt oder aber theoretisch plausibel zu erwarten ist. (Becker-Mrotzek et al. 2015, S. 84) $)^{11}$

\subsubsection{Deutschdidaktische Forschungsprojekte}

Rein deutschdidaktische Forschungsprojekte, die als DFG-Sachbeihilfe gewährt werden, sind herausragende Erfolge auf dem Hintergrund der bisherigen Forschungsentwicklung. Eines der ersten Projekte dieser Kategorie ist das seit 2013 laufende Projekt von Christian Dawidowski „Ko-Konstruktion von Bildungsvorstellungen im Verlauf der gymnasialen Oberstufe" (Deutungsmuster über Literatur bei Schülern der Sekundarstufe), das mit narrativen Interviews und der Videographie von Unterrichtssequenzen sowie mit der Analyse von Essays der Schüler arbeitet (vgl. Dawidowski, in Vorb.). ${ }^{12}$

11 Als interdisziplinäres und zugleich deutschdidaktisches Projekt ist die von 2009 bis 2015 vom BMBF geförderte Forschergruppe „Teilkomponenten der Schreibkompetenz" als weiteres Schreibprojekt zu nennen. (http://www.bmbf.schreibkompetenz.com). Ich danke Michael Becker-Mrotzek für seine Hinweise zum Stand beider Projekte.

12 Als weiteres Beispiel rein deutschdidaktischer von der DFG geförderter Einzelprojekte ist als kürzlich bewilligtes Projekt zu nennen: Irene Pieper/Dorothee Wieser: „Literarisches Verstehen im Umgang mit Metaphorik: Rekonstruktion von lernerseitigen Verstehensprozessen und lehrerseitigen Modellierungen (LiMet)“. 
Mit diesem Projekt ist ein literaturdidaktischer Projektantrag mit einer systematischen Fragestellung zum Zuge gekommen - dies war bislang nur mit fachhistorischen Fragestellungen möglich. ${ }^{13}$ Mit dem Konzept der literarischen Bildung kommt dieses Projekt auf die ureigene Fragestellung der Literaturdidaktik vor der Kompetenzorientierung sozusagen wieder zurück. Außerdem rückt das Projekt die Literaturrezeption in den Mittelpunkt, die in der Forschung zuvor als ein nachgeordneter Spezialfall von Lesekompetenz gegolten hat. Die dieser Fragestellungen angemessenen Untersuchungsmethoden sind die ,weichen 'Verfahren qualitativer empirischer Forschung.

Das Projekt „Ko-Konstruktion von Bildungsvorstellungen im Verlauf der gymnasialen Oberstufe" bezieht sich auf die Frage,

wie Bildungsvorstellungen (verstanden als Deutungsmuster und Wertvorstellungen literarischer Bildung von Schüler/innen und Lehrer/innen) wechselseitig im Sinne von Bestätigung, Ablehnung oder Variation konstruiert werden. Hierbei wird von einer KoKonstruktion im Rahmen des Unterrichtshandelns als einer interaktionstheoretischen Maßgabe ausgegangen. (Dawidowski, in Vorb.)

Das Profil literarischer Bildung wird als soziales, nicht als individuell privates Phänomen konturiert, wodurch der Befund des Auseinanderklaffens öffentlicher und privater Lektüre(-vorlieben) bestätigt wird.

Im Bereich der erfolgreichen deutschdidaktischen Schreibforschung ist das durch die VW-Stiftung im Rahmen des Programms „Deutsch Plus - Wissenschaft ist mehrsprachig" von 2012 bis 2016 geförderte Gießener Projekt zur Eristischen Literalität ${ }^{14}$ unter der Leitung von Helmuth Feilke und Katrin Lehnen zu erwähnen. Dieses Projekt belegt, welchen Spezifizierungs- und Elaborationsgrad die deutschdidaktische Schreibforschung erreichen kann. Es handelt sich um ein Projekt, das in einem der von Hurrelmann genannten ,Integrationsfelder' angesiedelt ist, in denen fachwissenschaftliche Fundierung und fachdidaktische Expertise Hand in Hand gehen müssen (s. o., Abschnitt 1). Das Thema der Eristischen Literalität ist - wie für die sog. ,Integrationfelder' kennzeichnend - noch dazu hochschulpolitisch und gesellschaftspolitisch bedeutsam, da es bei der Förderung ausländischer Studierender und bei Studierenden mit Migrationshintergrund

13 Vgl. dazu das u. a. von Bodo Friedrich und Harro Müller-Michaels geleitete DFGProjekt „Geschichte des Deutschunterrichts in beiden deutschen Staaten von 1945 bis 1989“ (http://homepage.ruhr-uni-bochum.de/harro.mueller-michaels/DFG/dfg.html, abgerufen am 25.11.2015).

14 Unter der Eristischen Literalität wird die Fähigkeit verstanden, „im Schreiben widerstreitende Positionen darzustellen und zugleich für die eigene Problembearbeitung zu nutzen“" (Feilke/Lehnen 2012). 
bedeutsam ist. ${ }^{15}$ Zwar ist die Modellbildung auch in anderen Lernbereichen fortgeschritten. Jedoch ermöglicht es die seit langem empirisch und interdisziplinär arbeitende Schreibforschung, dass man einzelne Schreibtextsorten exakt benennen, in Teilprozeduren zerlegen und Erwerbsbedingungen und Förderaspekte erläutern kann.

\section{Systematische Bewertung der Forschungsentwicklung seit den 90er Jahren}

Die zu Anfang gestellten Fragen nach dem Nutzen interdisziplinärer Konstellationen für die Deutschdidaktik, nach ihrer Bewertung sowie nach der Zukunft solcher Forschungsverbünde sind implizit schon beantwortet worden. Es ist keine Frage, dass die Deutschdidaktik als Disziplin in ihrer Repräsentanz an den Hochschulen, in ihrer Reputation und besonders bezogen auf den wissenschaftlichen Nachwuchs erheblich gewachsen ist, obwohl zugleich in allen angesprochenen Punkten noch Verbesserungsbedarf besteht. Zugleich sind zahlreiche Einzelforscher und ihre Teams gegenseitig anerkannt und es haben sich tragfähige Arbeitsbündnisse etabliert, die ihrerseits große Forschungsverbünde wie LiFT-2 und BiSS ermöglicht haben. So ist die Option für die Zukunft zweifellos die Fortsetzung interdisziplinärer und auch internationaler Verbundforschung. Durch die immer weiter ausgefeilte Modellierung und Ausarbeitung einzelner Faktoren des Lehrens und Lernens wie z. B. dem fachlichen Selbstkonzept und den epistemischen Überzeugungen wird eine präzisere Modellierung von Lehr-Lern-Prozessen möglich (vgl. Wieser 2012, S. 141f.; Winkler 2014).

Allerdings ist die Fortschreibung dieses Paradigmas nicht der einzige Weg, den die Deutschdidaktik der 10er und der 20er Jahre des 21. Jahrhunderts geht und in der Zukunft noch gehen wird. Sie erschöpft sich nicht in demjenigen Wissenschaftstyp, der auch hier in diesem Beitrag im Vordergrund stand: nämlich in der kompetenzorientierten quantitativen empirischen Forschung. Nach dem beispiellosen Erfolg der letzten 25 Jahre sollte sich die Deutschdidaktik wieder etwas kontemplativ und meditativ zurücklehnen, sich nach wie vor auch als Kulturwissenschaft mit einem weiten Themenspektrum begreifen und zu kreativen und innovativen Beobachtungen zurückkehren (vgl. Kepser 2013).

15 Ich danke Helmuth Feilke für wertvolle Hinweise zum Stand des Projekts. Ebenfalls auf das Schreiben in mehrsprachigen Kontexten ausgerichtet ist das schweizerische Weiterbildungsprojekt „Quims Schreibförderung“, das „zu drei Dimensionen der Schreibförderung - basale Schreibfertigkeiten, Schreibstrategien, Schreiben als soziale Praxis - einen vertieften Einblick und praktische Anregungen [gibt]“ (Lindauer/Sturm 2012). 
Neben dieser Ausrichtung als Kulturwissenschaft formiert sich in explizit forschungsbezogener Hinsicht eine selbstreflexive Richtung in der Deutschdidaktik. So beleuchtet Daniel Scherf zum Abschluss seiner Untersuchung über die Wirkung von Leseförderkonzepten bei der Lehrerschaft (selbst-)kritisch die Rezeption deutschdidaktischer Implementationen und stellt fest:

Die Lese- und Weiterbildungsdidaktik sollte sich dafür interessieren, was Studierende bzw. Lehrende außerhalb fachdidaktischer Wissensbestände zu Leseförderung wissen[,] und sie sollte thematisieren, wie ihre Wissensangebote in die komplexe Legierung professionellen Wissens eingehen, [...] da das immer noch verbreitete Ansinnen, nichtwissenschaftliche oder nicht innovative Wissensbestände durch wissenschaftliche einfach zu ,überschreiben, nur in den seltensten Fällen funktioniert. (Scherf 2013, S. 438)

Der Kern der hier vollzogenen Selbstreflexion besteht in der Rückbesinnung auf die eigene Disziplin und auf deren Fachlichkeit. Bei dieser Rückbesinnung kommt es nicht primär auf die Vernetzung und auf die interdisziplinäre Kooperation an, sondern vielmehr auf die Selbstvergewisserung der eigenen fachlichen Inhalte und wie sich fachliches (fachwissenschaftliches und fachdidaktisches) Wissen und Können zueinander verhalten. Dass Fachwissen hohe Relevanz für die Wirksamkeit von Professionshandeln hat, wurde zum ersten Mal in der COACTIV-Studie am Beispiel der Mathematik nachgewiesen (vgl. ausführlich z. B. Baumert/Kunter 2011). Auch in den hier referierten Untersuchungsreihen des TEDS-LT-Projekts spielt Fachwissen eine wichtige Rolle (s. o., Abschnitt 2.3). Während bei TEDS-LT das fachwissenschaftliche Wissen im Vordergrund steht, fungiert in der selbstreflexiven Deutschdidaktik das fachdidaktische Wissen als Fundament gerade für die Methodenentwicklung, die die Deutschdidaktik ja im Paradigma der interdisziplinären Verbundforschung aus der Hand gegeben hatte:

Fachdidaktische Methodenentwicklung hat sich als Grundlage fachangemessener empirischer Forschung auf einen fachbezogenen hermeneutischen „Denkrahmen“ (Ossner 2006a, 9) zu beziehen, der (fortwährend) zu hinterfragen und zu begründen ist, statt einen (neuen) Denkrahmen darzustellen (vgl. Ossner 2006b; Steinbrenner 2007, 6ff.); sie muss im Kontext fachlicher Vergewisserung erfolgen. (Scherf 2013, S. 441f.)

Aus diesem Zitat sind der ,fachbezogene hermeneutische Denkrahmen' (statt des fraglos vorgegebenen szientifischen Denkrahmens) und die ,fachliche Vergewisserung' als Eckpunkte, fachangemessener empirischer Forschung' hervorzuheben. Diese ,neue deutschdidaktische Unterrichtsforschung ist bestrebt, ihre Ergebnisse selbstreflexiv und selbstkritisch an die Praxis zu vermitteln. ${ }^{16}$ Dies begreift sie als eigenständige, aber der Forschung noch zugehörige Aufgabe.

16 Vgl. als von Scherf selbst genannte Beispiele Schmelz (2009) und Nix (2011). 
Während im Paradigma der Kompetenzorientierung die empirische Verifizierung von Forschungshypothesen dem unwiderrufbaren Nachweis der Richtigkeit und Wahrheit der zugrunde liegenden Aussagen entsprach und damit von dieser empirischen Verifizierung eine selbstverständliche Handlungsanweisung für die Praxis ausging, eine Art selbstredende Plausibilität, suchen deutschdidaktische Forscher dieser neuen Generation den Dialog mit den Akteuren vor Ort. Dieser Dialog - als Variante von Interdisziplinarität, die sich in diesem Fall zwischen Wissenschaft und Handlungsfeld vollzieht - hat Vorrang vor der Suche nach Arbeitsbündnissen innerhalb der Wissenschaft, in denen durchaus der kritische Dialog unter den Peers gepflegt wurde. Neben diesem Dialog gibt es ein Primat der programmatischen Elaboration der eigenen Fachlichkeit und ihrer Bedeutung für das Lehramtsstudium. Von diesem sich abzeichnenden Paradigma der selbstreflexiven Fachlichkeit in der deutschdidaktischen Unterrichtsforschung ist in der Zukunft noch einiges zu erwarten.

\section{Literatur}

Abraham, Ulf/Bremerich-Vos, Albert/Frederking, Volker/Wieler, Petra (Hrsg.) (2003): Deutschdidaktik und Deutschunterricht nach PISA. Freiburg i. Br.: Fillibach.

Baumert, Jürgen/Kunter, Mareike (2006): Stichwort: Professionelle Kompetenz von Lehrkräften. In: Zeitschrift für Erziehungswissenschaft. Jg. 9. H. 4, S. 469-520.

Baumert, Jürgen/Kunter, Mareike (2011): Das mathematikspezifische Wissen von Lehrkräften, kognitive Aktivierung im Unterricht und Lernfortschritte von Schülerinnen und Schülern. In: Kunter, Mareike/Baumert, Jürgen/Blum, Werner/Klusmann, Uta/Krauss, Stefan/Neubrand, Michael (Hrsg.): Professionelle Kompetenz von Lehrkräften. Ergebnisse des Forschungsprogramms COACTIV. Münster et al.: Waxmann, S. 163-192.

Bayrhuber, Horst (Hrsg.) (2012): Formate fachdidaktischer Forschung. Empirische Projekte - historische Analysen - theoretische Grundlegungen. Münster u. a.: Waxmann.

Becker-Mrotzek, Michael (1997): Zum Verhältnis von Sprachwissenschaft und Sprachdidaktik. In: Didaktik Deutsch. Jg. 2. H. 3, S. 16-32.

Becker-Mrotzek, Michael/Hasselhorn, Marcus/Roth, Hans-Joachim/Stanat, Petra (2015): Stichwort: Die Bund-Länder Initiative „Bildung durch Sprache und Schrift (BiSS)“. In: Psychologie in Erziehung und Unterricht. Jg. 63. H. 1, S. 75-77.

Belke, Eva/Dipper, Stefanie/Kandel, Sonia/Müller, Claudia (2015): Literacy as a key to social participation. Psycholinguistic perspectives on orthography instruction 
and literacy acquisition. http://staff.germanistik.rub.de/claudia-mueller/wp-content/uploads/Projektzusammenfassung.pdf Abgerufen am 30.06.2015.

Blömeke, Sigrid (2011): Teacher Education and Development Study: Learning to Teach (TEDS-LT). Erfassung von Lehrerkompetenzen in gering strukturierten Domänen. In: Blömeke, Sigrid/Bremerich-Vos, Albert/Haudeck, Helga/ Kaiser, Gabriele/Nold, Günter/Schwippert, Knut/Willenberg, Heiner (Hrsg.): Kompetenzen von Lehramtsstudierenden in gering strukturierten Domänen. Erste Ergebnisse aus TEDS-LT. Münster: Waxmann, S. 7-24.

Böttcher, Wolfgang/Blasberg, Sina (2015): Strategisch aufgestellt und professionell organisiert? Eine explorative Studie zu Strukturen und Status der Lehrerbildung. Berlin: Deutsche Telekom Stiftung und Stifterverband für die deutsche Wissenschaft. http://www.hrk.de/uploads/media/Studie_Querstrukturen.pdf. Abgerufen am 24.11.2015.

Bremerich-Vos, Albert/Dämmer, Jutta/Willenberg, Heiner/Schwippert, Knut (2011): Professionelles Wissen von Studierenden des Lehramts Deutsch. In: Blömeke, Sigrid/Bremerich-Vos, Albert/Haudeck, Helga/Kaiser, Gabriele/ Nold, Günter/Schwippert, Knut/Willenberg, Heiner (Hrsg.): Kompetenzen von Lehramtsstudierenden in gering strukturierten Domänen. Erste Ergebnisse aus TEDS-LT. Münster: Waxmann, S. 47-76.

Buchholtz, Christiane/Doll, Jörg/Stancel-Piatak, Agnes/Blömeke, Sigrid/Lehmann, Rainer/Schwippert, Knut (2011): Anlage und Durchführung der Studie TEDS-LT. In: Blömeke, Sigrid/Bremerich-Vos, Albert/Haudeck, Helga/ Kaiser, Gabriele/Nold, Günter/Schwippert, Knut/Willenberg, Heiner (Hrsg.): Kompetenzen von Lehramtsstudierenden in gering strukturierten Domänen. Erste Ergebnisse aus TEDS-LT. Münster: Waxmann, S. 25-45.

Bundesministerium für Bildung und Forschung (2013): Bund-Länder-Vereinbarung über ein gemeinsames Programm „Qualitätsoffensive Lehrerbildung“ gemäß Artikel 91 b des Grundgesetzes vom 12. April 2013. https://www.bmbf. de/files/bund_laender_vereinbarung_qualitaetsoffensive_lehrerbildung.pdf. Abgerufen am 24.11.2015.

Dawidowski, Christian (2009): Literarische Bildung in der heutigen Mediengesellschaft. Eine empirische Studie zur kultursoziologischen Leseforschung. Frankfurt a. M. u. a.: Lang.

Dawidowski, Christian (2016): Literarizität und literarische Bildung im Literaturunterricht: Eine empirische Annäherung. In: Brüggemann, Jörn/Dehrmann, MarkGeorg/Standke, Jan (Hrsg.): Literarizität. Herausforderungen für Theoriebildung, empirische Forschung und Vermittlung. Fachdidaktische und Fachwissenschaftliche Perspektiven. Baltmannsweiler: Schneider, S. 155-168.

Dawidowski, Christian/Hoffmann, Anna (in Vorb.): Ko-Konstruktion von literarischen Bildungsvorstellungen im Verlauf der gymnasialen Oberstufe. In: 
Mitterer, Nicola/Nagy, Hajnalka/Wintersteiner, Werner (Hrsg.): Die Ansprüche der Literatur und deren Vermittlung. Mitteilbares, Mittelbares und Unvermitteltes. Theoretische Perspektiven auf die Literaturdidaktik. Frankfurt a. M.: Lang.

Ehlich, Konrad/Valtin, Renate (2012): Wieder ein fragwürdiges Millionenprojekt zur Sprachforschung. Die Länder wollen wissen, ob sie für die Sprachaneignung das Richtige tun, werden es aber kaum erfahren. In: Frankfurter Allgemeine Zeitung, 05.12.2012.

Essen, Erika (1956): Methodik des Deutschunterrichts. Heidelberg: Quelle \& Meyer.

Feilke, Helmuth/Lehnen, Katrin (2012): Eristische Literalität. Erwerb und Ausbau wissenschaftlicher Textkompetenz im Deutschen. Adjungiertes Projekt des Instituts für Germanistik. Universität Gießen. https://www.uni-giessen.de/fbz/ zmi/projekte/eristischeliteralitaet. Abgerufen am 30.06.2015.

Frederking, Volker/Meier, Christel/Stanat, Petra/Dickhäuser, Oliver (2008): Ein Modell literarästhetischer Urteilskompetenz. In: Didaktik Deutsch. Jg. 13. H. 25, S. 11-31.

Frederking, Volker (2014): Deutschdidaktik als transdisziplinäre, anwendungsund grundlagenorientierte empirische Wissenschaft. In: Mitteilungen des Deutschen Germanistenverbandes. Jg. 61. H. 2, S. 109-119.

Fritzsche, Joachim/Krempelmann, Anita/Tosun, Claudia (2006): Literaturunterricht kontrastiv. Baltmannsweiler: Schneider Hohengehren.

Garbe, Christine/Holle, Karl/Weinhold, Swantje (2009): ADORE - Teaching Struggling Adolescent Readers. A Comparative Study of Good Practices in European Countries. LEUPHANA University of Lueneburg. http://www.leuphana.de/fileadmin/user_upload/Forschungseinrichtungen/Inst_Deutsch/ ADORE_Executive_Summary_15-06-09-1.pdf. Abgerufen am 07.05.2015.

Groeben, Norbert/Hurrelmann, Bettina (2006): Empirische Unterrichtsforschung in der Literatur- und Lesedidaktik. Ein Weiterbildungsprogramm (Lesesozialisation und Medien). Weinheim: Juventa.

Groeben, Norbert (Hrsg.) (1999): Lesesozialisation in der Mediengesellschaft: Zentrale Begriffsexplikationen. Köln: Psychologisches Institut.

Groeben, Norbert (2005): Auf dem Weg zu einer deutsch-didaktischen Unterrichtsforschung? In: Stückrath, Jörn/Strobel, Ricarda (Hrsg.): Deutschunterricht empirisch. Beiträge zur Überprüfbarkeit von Lernfortschritten im Sprach-, Literatur- und Medienunterricht. Baltmannsweiler: Schneider, S. 7-33.

Groeben, Norbert/Hurrelmann, Bettina (Hrsg.) (2004): Lesesozialisation in der Mediengesellschaft. Ein Forschungsüberblick. Weinheim: Juventa. 
Grzesik, Jürgen (1976): Die Steuerung von Lernprozessen im Unterricht. Heidelberg: Quelle \& Meyer.

Hurrelmann, Bettina (1998): Deutschdidaktik - kein Ort, nirgends? In: Didaktik Deutsch. Sonderheft, S. 13-39.

Hurrelmann, Bettina/Groeben, Norbert (2006): Forschungspraktikum. In: Groeben, Norbert/Hurrelmann, Bettina: Empirische Unterrichtsforschung in der Literatur- und Lesedidaktik. Ein Weiterbildungsprogramm (Lesesozialisation und Medien). Weinheim: Juventa, S. 529-535.

Ivo, Hubert (1977): Zur Wissenschaftlichkeit der Didaktik der deutschen Sprache und Literatur. Vorüberlegungen zu einer „Fachunterrichtswissenschaft“. Frankfurt a. M., Berlin/München: Diesterweg.

Kammler, Clemens/Knapp, Werner (Hrsg.) (2002): Empirische Unterrichtsforschung und Deutschdidaktik. Baltmannsweiler: Schneider.

Kepser, Matthis (2013): Deutschdidaktik als eingreifende Kulturwissenschaft. Ein Positionierungsversuch im wissenschaftlichen Feld. In: Didaktik Deutsch. Jg. 18. H. 34. S. 52-68.

Lindauer, Thomas/Sturm, Afra (2012): QUIMS Schreibförderung. Qualität in multikulturellen Schulen. Zentrum Lesen - Fachhochschule Nordwestschweiz. http:// www.fhnw.ch/personen/thomas-lindauer/projekte. Abgerufen am 30.06.2015.

Müller-Michaels, Harro (1994): Konzepte des Deutschunterrichts nach 1968. In: Hohmann, Joachim S. (Hrsg.): Deutschunterricht zwischen Reform und Modernismus. Blicke auf die Zeit 1968 bis heute. Frankfurt u. a.: Lang, S. 27-43.

Müller-Michaels, Harro/Rupp, Barbara (1978): Literatur im Alltag und Unterricht. Ansätze zu einer Rezeptionspragmatik. Kronberg: Scriptor.

Nickel-Bacon, Irmgard (2006): Positionen der Literaturdidaktik - Methoden des Literaturunterrichts. Ein heuristischer Explikationsversuch für die empirische Grundlagenforschung. In: Groeben, Norbert/Hurrelmann, Bettina (Hrsg.): Empirische Unterrichtsforschung in der Literatur- und Lesedidaktik. Ein Weiterbildungsprogramm. Weinheim: Juventa, S. 95-114.

Nix, Daniel (2011): Förderung von Leseflüssigkeit. Theoretische Fundierung und empirische Überprüfung eines kooperativen Lautlese-Verfahrens im Deutschunterricht. Weinheim: Juventa.

Nündel, Ernst/Schlotthaus, Werner (1978): Angenommen, Agamemnon. Wie Lehrer mit Texten umgehen. München u. a.: Urban \& Schwarzenberg.

Pieper, Irene/Rosebrock, Cornelia/Wirthwein, Heike/Volz, Steffen (2004): Lesesozialisation in schriftfernen Lebenswelten. Lektüre und Mediengebrauch von HauptschülerInnen. Weinheim: Juventa.

Pieper, Irene et al. (2012): Referenzrahmen Literaturalter 12-15Jahre. Literary Framework for Teachers in Secondary Education (LiFT-2). Schlüsseltexte: Handreichung 
zur Webseite. http://de.literaryframework.eu/; de.literaryframework.eu/keydocuments.html. Abgerufen am 09.03.2016.

Pieper, Irene (2014): Den Schüler vor Augen, den Anspruch im Sinn: Der internationale Referenzrahmen LiFT-2 zu Progression und Textauswahl für den Literaturunterricht der Sekundarstufen. In: Frederking, Volker/Huneke, HansWerner (Hrsg.): Taschenbuch des Deutschunterrichts. Band 3: Aktuelle Fragen der Deutschdidaktik. Baltmannsweiler: Schneider, S. 586-609.

Rosebrock, Cornelia/Nix, Daniel (2008): Grundlagen der Lesedidaktik und der systematischen schulischen Leseförderung. Baltmannsweiler: Schneider.

Rosebrock, Cornelia/Nix, Daniel/Rieckmann, Carola/Gold, Andreas (2011): Leseflüssigkeit fördern. Lautleseverfahren für die Primar- und Sekundarstufe. Seelze: Klett Kallmeyer.

Rothstein, Björn/Kröger-Bidlo, Hanna/Gräsel, Cornelia/Rupp, Gerhard (2014): Überlegungen zur Messung des Kohäsionsgrades von Texten. In: Linguistische Berichte. H. 237, S. 37-56.

Rupp, Gerhard/Bonholt, Helge (2006): Lehr-Lern-Forschung als empirische Lese-/Literaturdidaktik?! In: Groeben, Norbert/Hurrelmann, Bettina (Hrsg.): Empirische Unterrichtsforschung in der Literatur- und Lesedidaktik. Ein Weiterbildungsprogramm. Weinheim: Juventa, S. 239-253.

Rupp, Gerhard/Heyer, Petra/Bonholt, Helge (2004): Lesen und Medienkonsum. Wie Jugendliche den Deutschunterricht verarbeiten. Weinheim: Juventa.

Rupp, Gerhard/Gräsel, Cornelia (2012): Textkohäsion als Bedingung des Leseverständnisses am Beispiel der Verarbeitung von expositorischen und literarischen Texten. http://www.ruhr-uni-bochum.de/lidi/content/aktuelles.htm. Abgerufen am 10.03.2016.

Scherf, Daniel (2013): Leseförderung aus Lehrersicht. Eine qualitativ-empirische Untersuchung professionellen Wissens. Wiesbaden: Springer VS.

Schmelz, Markus (2009): Texte überarbeiten im Deutschunterricht der Hauptschule. Eine empirische Untersuchung zur Rezeption schreibdidaktischer Neuerungen. Baltmannsweiler: Schneider.

Schneider, Wolfgang/Baumert, Jürgen/Becker-Mrotzek, Michael/ Hasselhorn, Marcus/Kammermeyer, Gisela/Rauschenbach, Thomas/Roßbach, Hans-Günther/Roth, Hans-Joachim/Rothweiler, Monika/Stanat, Petra (2012): Expertise „Bildung durch Sprache und Schrift (BISS)“ (Bund-Länder-Initiative zur Sprachförderung, Sprachdiagnostik und Leseförderung). Bundesministerium für Bildung und Forschung. Berlin. https://www.bmbf.de/files/BISS_Expertise. pdf. Abgerufen am 09.03.2016.

Schulte-Berge, Gerlind/Schoett, Silja/Garbe, Christine (2002): Medienkompetenz und gesellschaftliche Handlungsfähigkeit von Jugendlichen im Lichte 
biographischer Forschung: zwei medienbiographische Fallstudien zum Zusammenhang von familiärer Gewalterfahrung und der Rezeption von Gewalt im Fernsehen. In: Groeben, Norbert/Hurrelmann, Bettina (Hrsg.): Medienkompetenz. Voraussetzungen, Dimensionen, Funktionen. Weinheim: Juventa, S. 255-268.

Shulman, Lee (1986): Those Who Understand: Knowledge Growth in Teaching. In: Educational Researcher. Vol. 15. No. 2, pp. 4-14.

Sieber, Peter (1998): Sprachwissenschaft und Sprachdidaktik - zum Verhältnis zweier ungleicher Schwestern. In: Beiträge zur Lehrerbildung. Jg. 16. H. 3, S. 353-366.

Terhart, Ewald (2002): Fremde Schwestern. Zum Verhältnis von Allgemeiner Didaktik und empirischer Lehr-Lern-Forschung. In: Zeitschrift für Pädagogische Psychologie. Jg. 16. H. 2, S. 77-86.

Ulshöfer, Robert (1957): Methodik des Deutschunterrichts. Mittelstufe II. Stuttgart: Klett.

Wieser, Dorothee (2012): Die Vermittlung fachlichen Wissens: Praktisches professionelles Wissen und epistemologische Überzeugungen. In: Pieper, Irene/ Wieser, Dorothee (Hrsg.): Fachliches Wissen und literarisches Verstehen. Studien zu einer brisanten Relation. Frankfurt a. M. u. a..: Lang, S. 135-152.

Winkler, Iris (2014): Durch die Brille der anderen sehen. Professionsbezogene Überzeugungen im Lehramtsstudium Deutsch. Antrittsvorlesung. Jena. 
Iris Winkler and Frederike Schmidt - 978-3-631-69285-1

Downloaded from PubFactory at 01/11/2019 10:32:53AM

via free access 
Volker Frederking, Sofie Henschel

\title{
Interdisziplinäre Forschung in der Deutschdidaktik am Beispiel des Projekts Literarästhetische Urteils- und Verstehenskompetenz (LUK)
}

\begin{abstract}
The paper presents empirical findings from the research project Literary Literacy that addressed the structure and validity of (literary) text comprehension. On this background we discuss chances and challenges of interdisciplinary research between Didactics of Literature Education and Educational Psychology.
\end{abstract}

\section{Fachdidaktiken zwischen Trans- und Interdisziplinarität (Volker Frederking)}

Fachdidaktiken lassen sich unter Zugrundelegung wissenschaftstheoretischer Kategorien von Jürgen Mittelstraß (1992) und Niklas Luhmann (1995) als transund interdisziplinär ausgerichtete Wissenschaften verstehen (vgl. Frederking 2014). Transdisziplinär sind die Fachdidaktiken, insofern sie als Disziplinen ihre Forschungen unter Einbeziehung von Methoden und Erkenntnissen der eigenen Fachwissenschaft, der Bildungswissenschaften, anderer Fachwissenschaften und anderer Fachdidaktiken betreiben. Sie überschreiten mithin permanent den engeren Horizont des eigenen disziplinären Kerns als Fachdidaktik, um in der Oszillation mit Erkenntnissen und Methoden anderer Disziplinen ihr spezifisches Forschungsfeld zu konturieren und ihren Forschungsfragen nachzugehen. Von diesem transdisziplinären Grundprofil der Fachdidaktiken ist ihre Offenheit zu interdisziplinärer Forschung zu unterscheiden. Während die Fachdidaktiken bei transdisziplinärer Forschung eine Art fortdauernder innerdisziplinärer Interdisziplinarität verwirklichen (vgl. Luhmann 1992, S. 458f.; Mittelstraß 1987, S. 156), weil sie zwar Methoden und Erkenntnisse anderer Disziplinen einbeziehen, aber für sich allein arbeiten, treten sie bei interdisziplinären Forschungen in unmittelbare Kooperation mit anderen Wissenschaften ein. Nach Niklas Luhmann (1992, S. 456f.) handelt es sich dabei um eine temporäre Form von Interdisziplinarität, in Abgrenzung zur okkasionellen. Okkasionelle Interdisziplinarität ergibt sich im Rahmen von Institutionen, Zeitschriften oder Kongressen - so auf unserer Jenaer Tagung „Fremde Schwestern“; temporäre Interdisziplinarität entsteht in vertiefter, 
aber zeitlich begrenzter Kooperation von mindestens zwei wissenschaftlichen Disziplinen im Kontext eines gemeinsamen Forschungsprojektes.

In der Deutschdidaktik haben interdisziplinäre Forschungskooperationen zwischen Fachdidaktik und empirischer Bildungswissenschaft, in denen die „Disziplinen auf Zeit“ (Mittelstraß 1992, S. 90) zusammenrücken, bereits eine gewisse Tradition - vor allem in der Sprachdidaktik und in der Lesedidaktik. Auch die vom IQB durchgeführten VERA-Erhebungen stellen für beide Bereiche gute Beispiele dar (vgl. Böhme, Stanat, Becker-Mrotzek, in diesem Band). In der Literaturdidaktik bilden interdisziplinäre Kooperationen hingegen noch immer die Ausnahme. Dies erklärt sich u. a. aus der hermeneutischen Verwurzelung der Literaturdidaktik, einem zuweilen ebenso ideologisch wie emphatisch überhöhten, dabei aber eigentümlich diffus bleibenden Literaturverständnis und einer spürbaren inneren Distanz gegenüber den in Naturwissenschaften wie Bildungswissenschaften zentralen empirischen Methoden. Vermittels quantitativer Verfahren den Umgang mit literarischen Texten im Literaturunterricht zu untersuchen, erscheint manchen Fachvertretern schlicht als nicht zu akzeptierender Tabubruch. Die damit verbundenen Reaktionsmuster sind entsprechend emotional und reichen vom Ignorieren bis zur polemischen Distanz. So wurde Jutta Wermkes (1989) bahnbrechende zweibändige Habilitationsstudie „Hab a Talent, sei a Genie!‘. Kreativität als paradoxe Aufgabe“ in der Disziplin schlicht nicht zur Kenntnis genommen, - nimmt man die Zahl der Zitationen zum Maßstab - während nicht empirisch abgesicherte, aber unterrichtspraktisch ausgerichtete Konzepte des kreativen Schreibens im Fach reüssierten. Das von der DFG geförderte Forschungsprojekt Literarästhetische Urteils- und Verstehenskompetenz (LUK), das Teil des von Ekkehard Klieme und Detlev Leutner initiierten und geleiteten Schwerpunktprogramms 1293 Kompetenzmodelle zur Erfassung individueller Lernergebnisse und zur Bilanzierung von Bildungsprozessen war, wurde hingegen zwar in anderen Fachdidaktiken und in den Bildungswissenschaften positiv rezipiert (vgl. z. B. Grünkorn/Fleischer/Klieme 2014; Hirsch 2014; Prediger 2014; Rolle 2011; Trautwein 2014), während sich in der Literaturdidaktik zunächst nur polemische Reflexe artikulierten, ehe ein wissenschaftlicher Diskurs einsetzte. Für diesen sind die beiden Tagungen zur Literarizität auf dem Monte Verita ebenso ein Beleg (Bertschi-Kaufmann/Rosebrock 2009, 2013) wie die in diesem Band dokumentierte Jenaer Tagung „Fremde Schwestern“. Nachfolgend sollen am Beispiel und mit Bezug auf das LUK-Projekt Möglichkeiten und Grenzen interdisziplinärer Forschung in der Literaturdidaktik reflektiert werden. 


\section{Die Interdisziplinarität des LUK-Projekts (Volker Frederking)}

Grundlage von LUK war und ist die interdisziplinäre Kooperation zwischen Vertreterinnen und Vertretern aus Literaturdidaktik auf der einen und Pädagogischer Psychologie und Erziehungswissenschaft auf der anderen Seite. Die besonderen Bedingungen dieser interdisziplinären Zusammenarbeit erklären sich mit Blick auf die unterschiedlichen Disziplinen, denen sie entstammen.

Beginnen wir mit der Pädagogischen Psychologie. Die Darstellung (Abb. 1) folgt Heinz Heckhausen (1987), in dessen Urteil die Psychologie bis ca. 1880/1900 durch eine Bi-Disziplinarität gekennzeichnet war, insofern sie einen hermeneutischen und einen empirischen Pol in sich vereinte. Spätestens mit Anbruch des 20. Jahrhunderts hat sich der empirische Pol dann allerdings im universitären Kontext als allein bestimmender durchgesetzt. Als empirische Teildisziplinen der Psychologie sind in der Grafik exemplarisch Entwicklungspsychologie (EP), Motivationspsychologie (MP), Lernpsychologie (LP) und Pädagogische Psychologie (PP) aufgeführt. Diese Wandlung der Psychologie zu einer rein empirisch arbeitenden Disziplin ging allerdings mit Verlustrechnungen einher. Denn die hermeneutischen Traditionen der Psychologie, vor allem die Psychoanalyse und ihre diversen Ausprägungen, wurden infolge dieses Prozesses aus dem universitären Bereich suspendiert. In der interdisziplinären Kooperation mit der Pädagogischen Psychologie arbeitet die Deutschdidaktik im Rahmen des LUK-Projekts mithin mit einem Partner zusammen, der sich nicht als hermeneutische, sondern als empirisch forschende Disziplin definiert.

Anders verhält es sich mit dem zweiten Partner, der empirischen Bildungsbzw. Erziehungswissenschaft (ohne Abbildung). Diese hat sich ebenfalls aus einer rein hermeneutisch ausgerichteten Disziplin, der Pädagogik, entwickelt und in Deutschland in den letzten Jahrzehnten als eigene, qualitativ wie quantitativ arbeitende Teildisziplin etabliert. Damit hat sich analog zur Psychologie auch im pädagogisch-erziehungswissenschaftlichen Bereich eine Bi-Disziplinarität herausgebildet; ob mit einer ähnlichen Fortsetzung wie in der Psychologie - d. h. einer Suspendierung der hermeneutischen Seite - bleibt abzuwarten.

Trotz mancher Ähnlichkeiten stellt sich die Genese der Deutschdidaktik Sprachdidaktik (SD), Literaturdidaktik (LD) etc. - als empirisch arbeitender Disziplin anders dar (vgl. Abb. 1). Zwar besitzt auch sie als Teildisziplin der Germanistik - Sprachwissenschaft (SW), Literaturwissenschaft (LW) etc. - einen vor allem hermeneutisch geprägten Ursprung. Und wie in der empirischen Erziehungs- bzw. Bildungswissenschaft hat sich auch die empirisch arbeitende Deutschdidaktik von der Fixierung auf diese Tradition gelöst. Allerdings hat sich bislang keine Bi-Disziplinarität in der Deutschdidaktik herausgebildet - und das wird m. E. auch in Zukunft nicht der Fall sein. Noch unwahrscheinlicher ist die 
Abspaltung. Anders als die Psychologie sollten nämlich weder germanistische Fachwissenschaft noch empirisch arbeitende Deutschdidaktik ihre hermeneutischen Wurzeln kappen. Aus meiner Sicht gilt, dass nicht die Substitution hermeneutischer durch empirische Ansätze der Weg der Deutschdidaktik als Disziplin ist, sondern die Integration bzw. fachspezifische Verschmelzung von beiden (vgl. Frederking 2003). Aus diesem Grund sehe ich die Deutschdidaktik als transdisziplinäre Wissenschaft, die hermeneutische und empirische Pole in sich vereint. Das war meine Position vor Beginn des LUK-Projekts - und das ist sie auch nach Abschluss des Projekts und mit Blick auf andere empirische Forschungsprojekte, die aktuell laufen bzw. in Vorbereitung sind.

Abb. 1: Fachgeschichtliche Hintergründe interdisziplinärer Kooperation zwischen Pädagogischer Psychologie und Literaturdidaktik

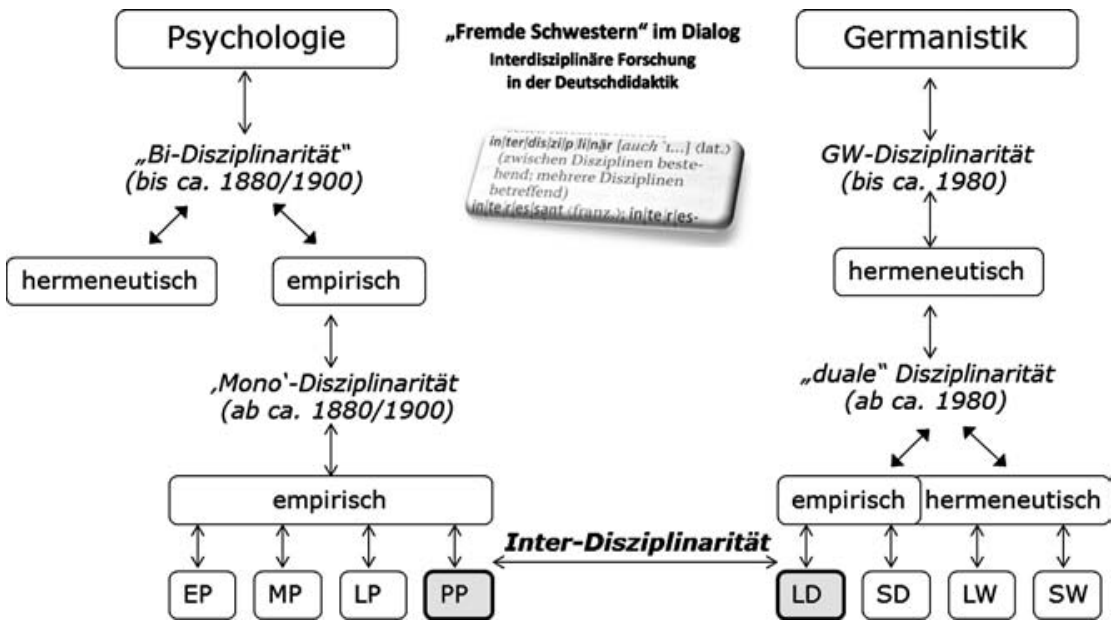

Dabei ist klar - Interdisziplinarität ist kein Abstraktum. Denn es begegnen sich nicht nur verschiedene Disziplinen und Wissenschaftskulturen, sondern auch verschiedene Menschen, fremde Schwestern und Brüder, um im Bilde zu bleiben. Und es bedarf Zeit, damit die gegenseitige Fremdheit der Disziplinen schwindet (bei den Menschen geht das in der Regel schneller). Denn wer interdisziplinär forscht, macht eine Erfahrung, die der Soziologe Franz-Xaver Kaufmann auf einem Forum zum Thema ,Interdisziplinarität` sehr präzise beschrieben hat:

Interdisziplinäre Kommunikation kann sich [...] gerade nicht auf jene Selbstverständlichkeiten verlassen, die die disziplinäre Kommunikation so sehr erleichtern: Die Gemeinsamkeiten der perspektivischen Grundannahmen und Auswahlgesichtspunkte, durch die 
das fachwissenschaftliche Erkenntnisinteresse geformt wird, die grundbegrifflichen Prämissen und die Eigenarten der fachwissenschaftlichen Methodik. (Kaufmann 1987, S. 70)

Interdisziplinarität setzt gemeinsame Forschungsinteressen voraus. Diese sind keinesfalls selbstverständlich und müssen sorgsam ausgelotet und bestimmt werden. Im Fall des LUK-Projekts standen in den drei von der DFG geförderten Projektphasen unterschiedliche Forschungsschwerpunkte im Fokus. Im kurzen Überblick:

LUK I (2007-09) hatte zum Ziel, in Abgrenzung zur allgemeinen Lesekompetenz ein theoretisch tragfähiges und operationalisierbares Kompetenzmodell zum literarischen Verstehen zu entwickeln und dieses empirisch zu überprüfen. Dazu wurde im Herbst 2008 eine Datenerhebung mit 1370 Schülerinnen und Schülern der 9. Klassenstufe von bayrischen Hauptschulen, Realschulen und Gymnasien durchgeführt.

LUK II (2009-11) zielte einerseits auf die genauere Untersuchung des literarischen Textverstehens bei schwächeren Lernenden an Hauptschulen. Andererseits wurde die längsschnittliche Entwicklung des literarischen Textverstehens bei Schülerinnen und Schülern von Realschulen und Gymnasien erforscht. Dazu wurden 1500 Jugendliche zu Beginn der 9. Klassenstufe untersucht sowie 1004 Jugendliche am Ende des 9. Schuljahres.

In LUK III (2011-13) wurde die längsschnittliche Untersuchung mit einer erneuten Erhebung von 964 Jugendlichen am Ende der 10. Klassenstufe fortgesetzt. Ein weiterer Fokus lag auf der Erweiterung des Modells literarischer Textverstehenskompetenz um die Aspekte des literarischen Fachwissens, der ästhetischen Aufmerksamkeit und der literarisch kodierten Emotionen.

In unserem Beitrag sollen zwei thematische Schwerpunkte herausgegriffen werden, um an ihnen Besonderheiten in der Kooperation und in den disziplinären und interdisziplinären Herausforderungen zu exemplifizieren.

\section{Inter-Disziplinäre Herausforderungen I: Zur Dimensionalität literarästhetischer Verstehenskompetenz (Volker Frederking)}

\subsection{Theoretische Ausgangspunkte und Grundlagen}

Am Anfang der LUK-Forschungen stand ein durch PISA und die deutschdidaktische Lesekompetenzforschung ausgelöster Weckruf. Stellvertretend sei auf Norbert Groeben verwiesen, der für das Lesekompetenz-Konzept des Schwerpunktprogramms Lesesozialisation in der Mediengesellschaft feststellte, es sei „nicht sinnvoll [...] zwischen dem Lesen literarischer und nicht-literarischer Texte zu unterscheiden“ (Groeben 2002, S. 12). Diese Position steht im Widerspruch zu der 
in der Literaturdidaktik weithin geteilten Grundannahme, dass mit literarischen Texten spezifische Verstehensansprüche (Mehrdeutigkeit, Unbestimmtheit, Verknüpfungsdichte etc.) verbunden sind (vgl. Zabka 2006) und es sich folgerichtig beim literarischen Verstehen um einen eigenen Kompetenzbereich handelt. Aber für diese Annahme fehlten die empirischen Belege. Zwar hatten Re-Analysen von PISA-Daten erste empirische Hinweise darauf ergeben, dass mit literarischem Verstehen spezifische Fähigkeitsaspekte verbunden sind (vgl. Artelt/Schlagmüller 2004). Diese psychometrisch ermittelten Anhaltspunkte basierten allerdings auf einem sehr begrenzten Textcorpus. Außerdem fehlte ein kompetenztheoretisches Fundament. Ein solches setzt entsprechende literaturdidaktische Modellierungen voraus, die im Rahmen von LUK entwickelt und in interdisziplinärer Kooperation mit den Bildungswissenschaften empirisch überprüft werden sollten. Entsprechend lautete die erste und wohl grundlegendste Forschungsfrage von LUK: Lässt sich literarästhetische Textverstehenskompetenz theoretisch wie empirisch von Lesekompetenz (bzw. faktualer Textverstehenskompetenz) abgrenzen?

Für den vorliegenden Zusammenhang kann zunächst genügen, sich nur auf die damit verbundenen literaturtheoretischen Modellierungen zu konzentrieren. Diesen lag die folgende Arbeitsdefinition zugrunde: Literarästhetische Textverstehenskompetenz bezeichnet die Fähigkeit, Literatur als textseitig intendiertes (Zusammen-)Spiel komplexer, oftmals mehrdeutiger Sinnstrukturen und Wirkungsabsichten auf inhaltlicher (semantischer) und formaler (idiolektaler) Ebene zu erfassen und auf dieser Basis kohärente Deutungen zu entwickeln respektive zu begründen (auch unter Berücksichtigung außertextueller Bezüge und Kontexte) (vgl. Frederking et al. 2011). Eine Operationalisierung auf Grundlage der in der Lesekompetenzforschung und bei PISA und IGLU favorisierten kognitionspsychologischen Modelle war dabei nicht möglich. Schließlich hatte Walter Kintsch, einer der exponiertesten Repräsentanten der Kognitionspsychologie, offen bekannt:

Zur literarischen Produktion gehört ein kreativer Aspekt, zum literarischen Verstehen ein ästhetischer. Die kognitiven Verstehensmodelle haben derzeit weder zur Kreativität noch zur Ästhetik etwas beizutragen. (Kintsch 1994, S. 44f.)

Kintschs wenig später formulierte Ergänzung - „Ein derartiges Projekt [wäre] nur in enger Zusammenarbeit mit Literaturwissenschaftlern möglich“" (Kintsch 1994, S. 49) - macht deutlich, dass innerhalb der Kognitionspsychologie die Notwendigkeit interdisziplinärer Zusammenarbeit mit text- bzw. literaturtheoretisch arbeitenden Wissenschaften schon früh gesehen wurde (vgl. dazu Krommer 2003). ${ }^{1}$

1 Auch in aktuelleren Publikationen hat sich an diesem Befund nichts Grundlegendes verändert (vgl. z. B. Kintsch 2000). Allerdings gibt es mittlerweile Versuche, auch 
Im Rahmen von LUK hat Kintschs Forderung insofern eine Umsetzung gefunden, als dass es hier - in Personalunion - Wissenschaftler aus Literaturwissenschaft und Literaturdidaktik (Christel Meier, Axel Krommer, Jörn Brüggemann, Volker Gerner, Lydia Steinhauer, Adelheid Rieder und ich selbst) waren, die gemeinsam versucht haben, die von Kintsch angedeuteten literaturtheoretischen Probleme zu lösen und auf dieser Basis mit Erziehungswissenschaft bzw. Pädagogischer Psychologie (Petra Stanat, Sofie Henschel, Thorsten Roick, Oliver Dickhäuser und Marcus Friedrich) eine empirische Überprüfung anzugehen. Allerdings wurden im Rahmen von LUK nicht - wie von Kintsch intendiert - die kognitiven Prozesse selbst untersucht, sondern deren Ergebnisse in Form von literarästhetischen Urteilen bzw. Verstehensresultaten.

Die damit verbundenen literaturtheoretischen Herausforderungen waren beträchtlich. Von ihrer Überwindung hing die Möglichkeit zur Operationalisierung und Erhebung literarischer Verstehenskompetenz im Rahmen der LUKForschungen ab. Dabei erwiesen sich die potenziellen literaturwissenschaftlichen Bezugspunkte für die Bewältigung dieser interdisziplinären Forschungsaufgabe als begrenzt. Denn das Ziel, literarische Textverstehenskompetenz empirisch zu untersuchen, setzt etwas voraus, was traditionell von vielen Richtungen in Literaturwissenschaft wie Literaturdidaktik bezweifelt wird: literarisches Textverstehen ist zumindest teilweise objektiv erfassbar. Wenn Paul de Man beispielsweise die These vertritt, dass jede Lektüre ein „fundamental misreading of literature“ darstelle (de Man 1971, S. 280), kann es nicht verwundern, dass eine Literaturdidaktik, die solche Positionen zu ihren primären Orientierungspunkten macht, angesichts der scheinbar unendlichen Re- und Dekonstruierbarkeit sinnhafter Bezüge von der Prämisse ausgehen zu müssen meint, dass das Wissen um das Nicht-Verstehen-Können von Literatur die höchste Erkenntnisstufe im Umgang mit Literatur darstelle (Härle 2011, Baum 2013) und "heute jede einigermaßen anschlussfähige Literaturtheorie“ lehre, der "Gegenstand Literatur" sei „nicht objektiv zu erfassen“ (Kammler 2006, S. 5). Allerdings gibt es in der Literaturwissenschaft durchaus Positionen und Ansätze, in denen plausibel gezeigt wird, dass verbindliche Bedeutungszuschreibungen im Zusammenhang mit literarischen Texten ebenso möglich sind wie klare und distinkte argumentative Begründungen von Verstehensurteilen. $\mathrm{Zu}$ nennen sind hier beispielsweise die methodologische Hermeneutik (Bühler 2003), literaturwissenschaftliche Applikationen der analytischen Philosophie (Fricke 1991; Jannidis et al. 2003) und die ästhetische Semiotik (Eco 1972, 1996, 1999, 2006).

kognitionspsychologisch im Bereich der Ästhetik zu forschen (vgl. z. B. Lüdtke/ Jacobs//Meyer-Sickendiek 2013). Diese lagen zu Projektbeginn aber noch nicht vor. 
Letztere wurde im Rahmen von LUK zum primären theoretischen Bezugspunkt gemacht, weil Umberto Ecos semiotischer Ansatz u. E. aktuell die komplexeste und umfassendste literaturtheoretische Modellierung darstellt, an die eine empirisch orientierte Literaturwissenschaft und Literaturdidaktik anknüpfen kann (vgl. Frederking et al. 2008). Anders als der durch Konstruktivismus, Dekonstruktivismus, Poststrukturalismus und Postmoderne geprägte literaturwissenschaftliche Mainstream trägt Eco nämlich einerseits der Deutungsoffenheit des Kunstwerks Rechnung und begründet und exemplifiziert andererseits literaturtheoretisch die Möglichkeit eines klar identifizierbaren Textsinns. Mit Ecos Theorie lässt sich deshalb eine Grundsatzfrage empirischer literaturdidaktischer Forschung im Bereich literarischen Verstehens beantworten. Diese lautet: Wie können trotz der Mehrdeutigkeit eines literarischen Textes eindeutig als ,richtig bzw. ,falsch zu klassifizierende Aussagen über ihn ermittelt werden?

Von zentraler Bedeutung ist in diesem Zusammenhang Ecos Drei-IntentionenModell und seine geisteswissenschaftliche Applikation von Poppers Falsifikationismus. Zur Erläuterung: Eco unterscheidet drei Intentionstypen, die den Bereich des Literarischen konturieren: die intentio auctoris, die Intention des Autors, die intentio lectoris, die Intention des Lesers, und die intentio operis, die Intention des Textes (1999, S. 35f.). Für die Ermittlung überprüfbarer Aussagen zu einem literarischen Text im Rahmen empirischer Forschung ist die Ebene der intentio operis von entscheidender Bedeutung - obschon Hans Harald Müller (2000) zu Recht Kritik an den anthropomorphen Konnotationen des Theorems vorgebracht hat. Denn auf der Ebene der intentio operis ist trotz der Offenheit des Kunstwerks (Eco 1962, 1996, 1999) und der damit verbundenen, semantischen Pluralität' (Eco 1962, S. 87) ein „kohärenter Textsinn“ (Eco 1999, S. 43) ermittelbar - und damit ein Ausschließen von nicht-adäquaten Deutungen.

Hier bezieht sich Eco explizit auf das Falsifikationsprinzip von Popper und unternimmt damit einen bemerkenswerten interdisziplinären Brückenschlag zu einem der maßgeblichen wissenschaftstheoretischen Bezugspunkte empirischer Forschung - einen Brückenschlag, dem wir im Rahmen von LUK gefolgt sind. Auf Popper geht die These zurück: „Theorien sind nicht verifizierbar; aber sie können sich bewähren“ (Popper 1935, S. 198). Und Theorien bewähren sich, wenn sie Falsifikationsversuchen standhalten. Das heißt praktisch:

Alles, was wir tun können, ist nach dem Falschheitsgehalt unserer besten Theorie zu fahnden. Das tun wir, indem wir sie zu widerlegen versuchen. (Popper 1972, S. 83)

Dieses Prinzip hat Eco literaturwissenschaftlich angewendet - und wir im Rahmen von LUK. In den Worten Ecos: 
Aufgrund welcher Kriterien entscheiden wir, ob eine Textinterpretation überzogen ist? [...] Ich meine [...], dass wir mit einem Popperschen Prinzip auskommen können: Wenn schon keine Regeln verbürgen, welche Interpretationen die ,besten' sind, dann lässt sich doch zumindest entscheiden, was ,schlecht' ist. (Eco 1996, S. 59)

Konkret bedeutet dies:

Zwischen der unergründlichen Intention des Autors und der anfechtbaren Intention des Lesers liegt die transparente Textintention, an der unhaltbare Interpretationen scheitern. (ebd., S. 87)

Die Unhaltbarkeit, d. h. die fehlende Verifizierbarkeit einer Deutung am Text - im Rahmen eines analytischen Urteils über einen inhaltlichen oder formalen Aspekt dieses literarischen Textes -, ist denn auch ein entscheidender Ansatzpunkt für die Operationalisierung von Testaufgaben gewesen. Dabei wurden die Lernenden zu Urteilen über den literarischen Text oder über Aussagen zu diesem herausgefordert. Diese Urteile und die darin zum Ausdruck kommenden literarästhetischen (Urteils-)Kompetenzen (=LUK) stellen den operationalisierbaren Teil literarästhetischer Verstehenskompetenz dar. Falsifikationismus im Rahmen von LUK bedeutet aber auch, dass Schülerinnen und Schüler z. B. in einem MultipleChoice-Item dazu herausgefordert werden, die zur Wahl gestellten Aussagen über einen literarischen Text - Attraktoren und Distraktoren - an diesem Text zu überprüfen und diejenigen Aussagen auszuschließen, die sich durch den Text falsifizieren lassen. LUK-Items fordern in diesem Sinne oft auch zur „Falsifizierung der Fehlinterpretationen“ (Eco 1999, S. 51) heraus. Dieses Vorgehen hat sich im Rahmen des Projektes bewährt, um das eingangs skizzierte literaturtheoretische Grundproblem, wie sich nämlich trotz Mehrdeutigkeit eines literarischen Textes Eindeutigkeit im Urteil herstellen lässt, zu lösen.

\subsection{Theoretische Modellierung und Operationalisierung des Konstrukts}

Auf der Grundlage dieser literatur- und wissenschaftstheoretischen Rahmung erfolgte in LUK die Konstruktmodellierung. Dabei war eine zweite Forschungsfrage leitend: Lässt sich literarästhetische Textverstehenskompetenz als ein mehrdimensionales Konstrukt theoretisch modellieren und empirisch bestätigen? Bei dieser von Seiten der Literaturdidaktik zu leistenden Entwicklungsarbeit lag auf Basis literaturwissenschaftlicher Theoreme und curricularer sowie unterrichtlicher Traditionen ein dreidimensionales Konstrukt nahe. Mit Bezug auf die Ebene der intentio operis wurden entsprechend drei Dimensionen literarästhetischer 
Textverstehenskompetenz theoretisch modelliert (vgl. zum Folgenden ausführlicher Frederking/Roick/Steinhauer 2011):

1. Semantische literarästhetische Textverstehenskompetenz

2. Idiolektale literarästhetische Textverstehenskompetenz

3. Kontextuelle literarästhetische Textverstehenskompetenz

Ad 1) Semantische literarästhetische Textverstehenskompetenz ist definiert als die Fähigkeit zum Erschließen zentraler Inhalte, Sinnstrukturen und Deutungsspielräume eines literarischen Textes. Sie entspricht in literaturtheoretisch fundierter Form dem, was in den Bildungsstandards mit der Formel „wesentliche Elemente eines Textes erfassen“ und „zentrale Inhalte erschließen“ (KMK 2004, S. 14) gemeint ist.

Mit Eco wird dabei unter interpretativem Erfassen „die semantische Aktualisierung“ dessen verstanden, „was der Text (als Strategie) durch die Mitarbeit eines Modell-Lesers zum Ausdruck bringen will“ (Eco 1998b, S. 226). Im Unterschied zur Unbegrenztheit der Sinnzuschreibungen des Lesers, wie sie konstruktivistisch impliziert ist, werden die Deutungsspielräume des empirischen bzw. realen Lesers im Rahmen der LUK-Modellierungen durch die Anforderungen des Textes an den idealen bzw. Modell-Leser (Eco 1999, S. 43) als begrenzt angesehen. Auf dieser Basis sind Operationalisierungen von Verstehensaussagen bzw. -urteilen möglich.

Ad 2) Die idiolektale Ebene bezeichnet die Fähigkeit zum Erfassen der formalen Spezifika eines literarischen Textes und ihrer ästhetischen Funktion. In den Bildungsstandards finden diese Aspekte in Formulierungen wie „wesentliche Fachbegriffe zur Erschließung von Literatur kennen und anwenden“ und „sprachliche Gestaltungsmittel in ihren Wirkungszusammenhängen und in ihrer historischen Bedingtheit erkennen“ (KMK 2003, S. 14) eine Entsprechung. Während bei PISA und VERA Formaspekte keine bzw. kaum eine Rolle gespielt haben bzw. spielen, werden sie im Rahmen von LUK als zweite Teildimension literarischer Textverstehenskompetenz angesehen. Zugrunde liegt Ecos Bestimmung des Idiolekts als ,strukturalem Schema' (Eco 1972, S. 151f.) eines ästhetischen Textes. Für dessen Erfassung besitzt die „ästhetische Funktion“ poetischer Sprache zentrale Bedeutung. Diese zeigt sich in „Mehrdeutigkeit“ und „Autoreflexivität“ und konkretisiert sich in der Frage, „, aufgrund welcher Strukturmerkmale der Text diese (oder andere) semantische Interpretationen hervorbringen" kann (Eco 1999, S. 43).

Ad 3) Die Ebene des kontextuellen literarästhetischen Urteilens bzw. Verstehens bezeichnet die Fähigkeit zum Erfassen außertextueller Bezüge (Werk, Autor, Gattung, Epoche, Kultur, Philosophie, Motiv- bzw. Mentalitätsgeschichte etc.). Auch diese modellierte Teildimension literarischer Verstehenskompetenz wurde bei PISA und VERA nicht bzw. kaum berücksichtigt, während sie in den Bildungsstandards 
zentral gewichtet ist, beispielsweise wenn es dort heißt, dass Lernende in der Lage sein sollen, „Zusammenhänge zwischen Text, Entstehungszeit und Leben des Autors/der Autorin bei der Arbeit an Texten aus Gegenwart und Vergangenheit herstellen" (KMK 2003, S. 14) zu können. In den Bildungsstandards für die Allgemeine Hochschulreife ist dieser Bereich ebenfalls zentral gewichtet (vgl. KMK 2012, S. 21; vgl. dazu Frederking/Wieser 2015, S. 186). Inhaltlich bestimmt und theoretisch fundiert ist diese dritte im Rahmen von LUK angenommene Teildimension literarischer Verstehenskompetenz durch eine ganze Reihe literaturwissenschaftlicher Ansätze: Gérard Genettes (1987) Theorie des Paratextes, Eric D. Hirschs (1967), Umberto Ecos (1999) und Fotis Jannidis' et al. (1999) Theorieansätze zum Autor bzw. zur Autorschaft, Gregory Curries (1990) Theorie der Fiktion, Julia Kristevas (1972) und Karlheinz Stierles (1984) Theorieansätze zur Intertextualität, Wilhelm Voßkamps (1992) Überlegungen zur Gattungsfrage und Rainer Rosenbergs (1992) Aufarbeitung des Epochendiskurses.

\subsection{Die Ergebnisse im metatheoretischen literaturdidaktischen Blick}

Nimmt man die theoretischen Modellierungen von LUK in einer Meta-Perspektive in den Blick, zeigt sich etwas für das Selbstverständnis der Literaturdidaktik als wissenschaftlicher Disziplin sehr Interessantes. Denn bei einem Theorieentwurf, wie er unter 3.2 in Grundlinien skizziert wurde - detailliertere Herleitungen und Begründungen finden sich an anderer Stelle (vgl. z. B. Frederking et al. 2011) würde eine traditionelle, rein hermeneutisch ausgerichtete Literaturdidaktik stehen bleiben, das Konzept zur Diskussion stellen und zu unterrichtspraktischen Modellierungen ansetzen. Für eine empirisch forschende, d. h. evidenzorientierte Literaturdidaktik endet hier hingegen lediglich die 1. Phase des Entwicklungsprozesses, die disziplinäre. Es schließt sich die 2. Phase, die interdisziplinäre, an (solange es noch keine eigene deutschdidaktische Expertise in hochwertiger quantitativer Forschung gibt) - und damit der Übergang von der Theoriebildung zur empirischen Theorieprüfung. In diesem Sachverhalt treten exemplarisch Merkmale jenes Paradigmenwechsels innerhalb unserer Disziplin ins Blickfeld, die mit der empirischen Wende verbunden sind. Denn eine empirische Theorieprüfung ist innerhalb der Literaturdidaktik etwas qualitativ Neues - empirische Grundlagenforschung in fachspezifischer Perspektive. Diese kann Ausgangspunkt für empirische literaturdidaktische Anwendungsforschung sein - wie in unserem Forschungsprojekt zur ästhetischen Kommunikation im Literaturunterricht ÄSKIL gezeigt werden konnte (vgl. Albrecht/Hornberger 2014; Brüggemann/Frederking 2015; Drewes/Albrecht 2014; Frederking et al. 2012b, 2015). 
Stellt schon die Verbindung von Theoriebildung und empirischer Theorieprüfung im Rahmen der Literaturdidaktik ein Novum dar, erschließen sich mit den Ergebnissen der empirischen Erhebungen in LUK I und den sich daraus ergebenden Konsequenzen noch weitaus bemerkenswertere Aspekte. Dies gilt schon für die Beantwortung der ersten Forschungsfrage. In allen drei von der DFG geförderten Phasen des Projekts ist sie immer wieder mit untersucht worden und jeweils haben die gewonnenen Daten deutliche empirische Hinweise darauf ergeben, dass literarisches Textverstehen tatsächlich eine eigene, von allgemeiner Lesekompetenz bzw. faktualem Textverstehen zu unterscheidende Teilkompetenz darstellt (vgl. auch Frederking/Roick/Steinhauer 2011, Frederking et al. 2012a; Meier et al., im Druck; Roick et al. 2010, 2013; Roick/Henschel 2015) - ein Beispiel für den möglichen Wandel der Literaturdidaktik von einer glaubensgestützten zu einer evidenzorientierten Disziplin.

Für die Weiterentwicklung des disziplinären Selbstverständnisses sind die Befunde zur zweiten Forschungsfrage aber noch interessanter. Die literaturdidaktischen Mitglieder des LUK-Teams waren sich nämlich aus theoretischer Perspektive relativ sicher, dass sich alle drei modellierten Teildimensionen literarischer Verstehenskompetenz empirisch abbilden würden - die beiden von Eco abgeleiteten Teildimensionen des Semantischen und des Idiolektalen ebenso wie die auf Basis mehrerer Theorien entwickelte Teildimension des Kontextuellen. Allerdings war uns bewusst, dass es aus literaturtheoretischer Sicht auch durchaus plausibel ist, Kontexte den anderen beiden Kompetenzdimensionen zuzurechnen, weil diese entweder eine semantische (z. B. Autorbezug) oder eine idiolektale (z. B. Gattungsbezug) Prägung aufweisen.

Die Ergebnisse der Modellprüfung sollten hier Klärung bringen (vgl. dazu im Detail Frederking/Roick/Steinhauer 2011; Frederking et al. 2012a; Roick et al. 2010). Verglichen wurden durch Item-Bündelung zunächst ein eindimensionales Modell, bei dem alle entwickelten Items undifferenziert unter einer Kompetenzdimension subsumiert wurden, mit dem favorisierten dreidimensionalen LUK-Modell aus semantischem, idiolektalem und kontextuellem Bereich und anschließend mit einem zweidimensionalen LUK-Modell, bei dem die Kontextitems der semantischen und der idiolektalen Dimension zugeordnet wurden. Für die Modellanalyse ist die Deviance, ein Maß zur Erfassung der Modellgüte, von entscheidender Bedeutung. Dabei gilt: Je geringer die Deviance, desto besser bildet das Modell die empirischen Daten an. Die Deviance liegt beim dreidimensionalen Modell bei 38918.96 und damit fast 30 Punkte unter dem eindimensionalen Modell. Das dreidimensionale Modell zeigt also tendenziell eine bessere Anpassung an die Daten als das eindimensionale Modell. Eine noch bessere Passung zeigt 
allerdings das zweidimensionale Modell. Die Deviance beträgt hier 38905.28 und bewegt sich damit noch einmal 13 Punkte unter dem dreidimensionalen Modell. Dies deutet von empirischer Seite darauf hin, dass ein zweidimensionales Modell dem dreidimensionalen Modell überlegen ist. Mit anderen Worten: die semantische bzw. idiolektale Prägung der Kontexte scheint schwerer zu wiegen als der Sachverhalt, dass es sich um textexterne Aspekte handelt (vgl. Frederking/Roick/ Steinhauer 2011; Roick et al. 2010). ${ }^{2}$ So tritt mit den ermittelten Befunden ein für eine literaturtheoretische Meta-Reflexion interessanter Modellfall ins Blickfeld: eine fachdidaktische Theorie wird empirisch überprüft und eine theoretische Frage - ob ein zwei- oder ein dreidimensionales Modell besser passt - empirisch ,geklärt.' ${ }^{3}$ Eine solche Theorieprüfung auf empirischer Basis ist innerhalb von Naturwissenschaften, Medizin oder Psychologie die Regel, für die Literaturdidaktik stellt sie eine als durchaus paradigmatisch zu verstehende Wende dar. Diese wäre ohne interdisziplinäre Zusammenarbeit nicht möglich gewesen.

2 Allerdings deuten neuere Untersuchungen, die insbesondere im Rahmen der dritten Projektphase vorgenommen wurden (vgl. Meier et al., im Druck), darauf hin, dass neben dem Semantischen und dem Idiolektalen weitere Subfacetten berücksichtigt werden müssen, um Kompetenzanforderungen des literarischen Textverstehens differenziert zu beschreiben.

3 Aus literaturdidaktischer Sicht besteht im Hinblick auf den kontextuellen Bereich aber noch weiterer Forschungsbedarf, da einige Items trotz des mehrstufigen ItemEntwicklungsverfahrens (Aufgabenentwicklung, Aufgabendiskussion und erste Aufgabenrevision, Cognitive Lab und zweite Aufgabenrevision, Pilotierung und dritte Aufgabenrevision) aus theoretischer Perspektive Optimierungsbedarf aufweisen. Im Rahmen des LUK Projektes konnte dieser Aspekt allerdings aus antragsstrategischen Gründen zunächst nicht weiter verfolgt werden.

Der weitere Forschungsbedarf im Hinblick auf die Kontext-Dimension ist allerdings nicht als Indikator für eine potenzielle oder tatsächliche Unzuverlässigkeit quantitativer empirischer Forschung misszuverstehen. Die quantitativen Methoden sind das Beste, was die Wissenschaft bislang zu bieten hat, weil sie eine Annäherung an Evidenz ermöglichen (Pant 2014). Allerdings ist angesichts der erkenntnistheoretischen Grenzen wissenschaftlicher Gewissheit (vgl. dazu das Popper-Zitat in der in Abschnitt 2.1 angeführten Textstelle) die in der empirischen Bildungsforschung verbreitete Rede von der ,Evidenzbasierung' bzw. ,Evidenzbasiertheit' missverständlich, weshalb ich selbst die Bezeichnung ,Evidenzorientierung' für zutreffender halte und vorschlage, von einer evidenzorientierten empirischen Forschung im Bereich der Fach- bzw. Literaturdidaktik zu sprechen. 


\section{Inter-Disziplinäre Herausforderungen II: Empirische Überprüfung bzw. Validierung des Konstrukts (Sofie Henschel)}

Im vorangegangenen dritten Abschnitt wurden zwei zentrale Forschungsfragen des Projekts, die die Struktur des literarischen Textverstehens betreffen, aufgeworfen und aus einer literaturdidaktischen Perspektive reflektiert. Insbesondere die zweite Forschungsfrage, die auf die Untersuchung der internen Struktur des literarischen Textverstehens abzielt, setzt voraus, dass mit hinreichender Sicherheit angenommen werden kann, dass für das Verstehen literarischer und nicht-literarischer (faktualer bzw. expositorischer) Texte (teilweise) unterschiedliche Kompetenzen benötigt werden. Unter Bezugnahme auf die Theorie der ästhetischen Semiotik (vgl. Eco 1999) wurden aus literaturdidaktischer Perspektive zunächst theoretische Verstehensanforderungen beschrieben, durch die sich das literarische Textverstehen auszeichnen könnte. Dies erfolgte nicht in Abgrenzung zu kognitiven Verarbeitungsprozessen, die für das nicht-literarische (expositorische) Textverstehen kennzeichnend sein könnten. Aus welchen theoretischen Gründen eine getrennte Betrachtung von Kompetenzen zum Verstehen literarischer und nicht-literarischer (faktualer) Texte überhaupt sinnvoll und angemessen sein sollte, ist anhand der dargestellten literaturdidaktischen Argumentationslinie, die primär texttheoretisch ansetzt, aus einer erziehungswissenschaftlich-psychologischen Perspektive nicht unmittelbar erkennbar.

Auch stellen die angesprochenen Ergebnisse der strukturellen Analysen, über die auszugsweise in Abschnitt 3 berichtet wurde, noch keine ausreichend verlässliche Grundlage dar, um mit hinreichender Sicherheit von einer zweidimensionalen Struktur des Textverstehens ausgehen zu können (vgl. Roick/Henschel 2015). Tatsächlich liefern Ergebnisse struktureller Analysen, wie sie im dritten Abschnitt berichtet wurden, nur eine erste Stützung für die angenommene Mehrdimensionalität. Die Spezifität der identifizierten Kompetenzfacetten, also des literarischen und nicht-literarischen bzw. faktualen Textverstehens, muss aber unbedingt durch weitere Studien gestützt werden, um eine Grundlage für Analysen zu schaffen, die eine genauere Untersuchung der internen Struktur des literarischen Textverstehens (also semantisch vs. idiolektal vs. kontextuell) sinnvoll erscheinen lassen. In Abschnitt 3 wurden bereits erste Ergebnisse zu Strukturanalysen berichtet, die die Dimensionalität des literarischen Textverstehens betreffen. Damit war die implizite Annahme verbunden, dass literarisches und expositorisches Textverstehen zwei voneinander unterscheidbare Kompetenzfacetten darstellen. Ob eine solche differenzierte Betrachtung aus theoretischer und empirischer Sicht plausibel bzw. angemessen ist, wurde im LUK Projekt anhand eines mehrstufigen Verfahrens 
untersucht. Nachfolgend wird in Auszügen veranschaulicht, wie komplex ein Validierungsprozess aus erziehungswissenschaftlich-psychologischer Perspektive ausfallen sollte, um die zweidimensionale Kompetenzstruktur des Textverstehens (literarisch vs. faktual) zu prüfen, die die Voraussetzung für die weitere Erforschung der Struktur und Verarbeitungsprozesse des literarischen Textverstehens darstellt.

\subsection{Theoretische Annahmen und empirische Befunde zu Verstehensanforderungen und kognitiven Verarbeitungsprozessen beim literarischen und faktualen Textverstehen}

Disziplinübergreifend besteht weitgehender Konsens darin, dass das Verstehen von Texten einen interaktiven Prozess darstellt, der durch (kognitive, motivationale und affektive) Merkmale des Lesers sowie textinterne Faktoren (z. B. Textstruktur, Mehrdeutigkeit) und textexterne Faktoren (Lesesituation, Leseinstruktion) beeinflusst wird (vgl. McNamara/Magliano 2009). Durch das Zusammenwirken dieser Faktoren aktivieren Leser sogenannte „Lesemodi“, d. h. sie nutzen u. a. textartspezifische (literarische vs. nicht-literarische/expositorische) kognitive (und affektive) Verarbeitungsstrategien, um eine mentale Repräsentation über das Gelesene aufzubauen (vgl. Meutsch 1987; Zwaan 1993). Bisherige empirische Studien weisen $u$. a. darauf hin, dass ein literarischer Lesemodus im Vergleich zu einem eher nicht-literarischen bzw. faktualen Lesemodus durch die Dominanz von Bottom-up-Prozessen, einen stärkeren Einbezug sprachlicher Aspekte bei der Bedeutungskonstruktion, die Bildung spezifischer Inferenz- und Elaborationstypen, eine höhere Relevanz affektiver Prozesse und den verzögerten Aufbau der situativen Textrepräsentation gekennzeichnet ist (vgl. z. B. Bourg 1996; Eva-Wood 2004; Fialho 2007; Meutsch 1987; Zwaan 1993). Diese Befunde wurden u. a. im Rahmen kognitionspsychologischer und psycholinguistischer Studien erbracht. Hinweise auf textartspezifische Verarbeitungsprozesse liefern aber auch neurowissenschaftliche Zugänge. So konnte etwa gezeigt werden, dass beim Lesen fiktionaler Geschichten Hirnareale aktiviert werden, die auch für empathische Prozesse relevant sind. Beim Lesen faktualer Texte waren diese Bereiche hingegen inaktiv (vgl. Altmann et al. 2014). Die Ergebnisse dieser Studien legen insgesamt nahe, dass Leser in Abhängigkeit des zugrunde liegenden Lesemodus (z. B. literarisch vs. nicht-literarisch bzw. faktual) und der damit assoziierten Verarbeitungsprozesse unterschiedliche Kompetenzen für den Aufbau einer mentalen Textrepräsentation benötigen dürften. Diese Annahme wird implizit auch in den Bildungsstandards für die Sekundarstufe vertreten, in denen die Förderung der literarischen Verstehenskompetenz im Unterricht neben der faktualen Verstehenskompetenz einen 
eigenständigen Schwerpunkt bildet (KMK 2004, S. 14). Zugleich ist anzunehmen, dass durch den Umgang mit literarischen Texten aufgrund ihrer besonderen Beschaffenheit und Funktion spezifische Unterrichtsziele, wie beispielweise die Ausbildung von Empathie, Fremdverstehen und die Persönlichkeitsentwicklung (KMK 2004, S. 9), besonders gut erreicht werden könnten.

Während die zuvor erwähnten Studien aus der Kognitionspsychologie, der Psycholinguistik und der Neurowissenschaft insgesamt relativ einvernehmlich auf textartspezifische Verarbeitungsprozesse hinweisen, kommen Analysen, die das Verstehensprodukt bzw. Verständnis von literarischen und expositorischen Texten betrachten, trotz ähnlicher Befunde zu unterschiedlichen Ergebnisinterpretationen. Artelt und Schlagmüller (2004) schlussfolgern auf Basis ihrer Reanalyse der PISA 2000-Daten, „dass der kompetente Umgang mit literarischen Texten als ein separater Teilaspekt der Lesekompetenz verstanden werden sollte“ (ebd., S. 179), weil der latente Zusammenhang zwischen dem literarischen Textverstehen und dem Verstehen faktual-kontinuierlicher bzw. faktual-diskontinuierlicher Texte deutlich niedriger ausfällt $(\mathrm{r}=.76 / 79)$ als zwischen den beiden faktualen Verstehensformen $(\mathrm{r}=.90)$. Böhme und Robitzsch (2009) kommen auf Grundlage ihrer strukturellen Analysen im Rahmen der Pilotierung des Grundschul-Ländervergleichs des IQB für die 3./4. Klassenstufe zu vergleichbaren Zusammenhängen zwischen literarischem und faktual-diskontinuierlichem Textverstehen $\left(r_{3 . \mathrm{KL}}=.74 / r_{4 . \mathrm{KL}}=75\right)$ und etwas höheren Zusammenhängen zwischen literarischem und faktual-kontinuierlichem Textverstehen $\left(r_{3 . \mathrm{KL}}=.84 / r_{4 . \mathrm{KL}}=85\right)$. Sie empfehlen auf Basis dieser Ergebnisse „bei der Modellierung der Lesekompetenz in sinnvoller Näherung mit eindimensionalen Modellen zu arbeiten“ (ebd., S. 277). Die Ergebnisse machen deutlich, dass strukturelle Analysen allein trotz vergleichbarer Befunde zu unterschiedlichen Ergebnisinterpretationen führen können und somit keine hinreichende Beurteilung der Validität des zugrunde liegenden Kompetenzstrukturmodells erlauben (vgl. Roick/Henschel 2015). Auch die in Abschnitt drei angesprochenen Ergebnisse der Strukturanalysen aus dem LUK Projekt (vgl. auch Frederking et al. 2012a; Roick et al. 2013) liefern folglich lediglich erste Anhaltspunkte für eine Mehrdimensionalität des Textverstehens im Allgemeinen (literarisch vs. faktual).

\subsection{Vorgehen zur Validierung von Kompetenzstrukturmodellen}

Die Validität stellt neben der Objektivität und der Reliabilität ein zentrales Gütekriterium dar und bildet ein integriertes bewertendes Urteil darüber ab, welche Schlussfolgerungen auf Basis eines Erhebungsinstrumentes bezüglich Bedeutung, Interpretation und Handlung zulässig sind (Hartig/Frey/Jude 2007). Um die Validität einer angenommenen Kompetenzstruktur hinreichend beurteilen zu können 
und Entscheidungsinkonsistenzen zu vermeiden, wie sie beispielsweise aus den strukturellen Analysen der PISA Daten und der Pilotierung des IQB-Ländervergleichs resultieren, empfiehlt sich ein mehrstufiges, hypothesenorientiertes Vorgehen, welches im Rahmen des LUK Projektes angewendet wurde (vgl. Roick/ Henschel 2015). Hierbei wird zunächst die dimensionale Struktur der angenommenen Kompetenz im Rahmen struktureller Analysen identifiziert (Stufe I: strukturelle Validierung), wie dies in den zuvor genannten Schulleistungsstudien und in Abschnitt 3 erfolgte. Typischerweise werden dazu hierarchische und theoretisch plausible Alternativmodelle (z. B. ein- vs. zwei- vs. dreidimensional) anhand einer Prüfstatistik (z. B. $\chi^{2}$-Differenzentest auf Basis der Deviance) miteinander verglichen. Die komplexere Modellvariante mit einer höheren Anzahl an Dimensionen wird gegenüber einem einfacheren Modell nur dann bevorzugt, wenn es die empirischen Daten signifikant besser abbildet. Die Analysen zur strukturellen Validierung, die wir im LUK Projekt durchführten, wiesen darauf hin, dass das Verstehen literarischer und nicht-literarischer bzw. faktualer Texte unterscheidbare Kompetenzdimensionen darstellen (vgl. z. B. Frederking et al. 2012a; Roick et al. 2013). Die Befunde unterstützen also die Ergebnisse, die anhand der PISA 2000 Daten gezeigt wurden (vgl. Artelt/Schlagmüller 2004). Da dieser Befund damit aber auch im Widerspruch zu der Ergebnisinterpretation von Böhme und Robitzsch (2009) sowie eindimensionaler theoretischer Auslegungen stehen, die z. B. in der deutschdidaktischen Forschung vertreten werden (z. B. Groeben 2002, S. 12), muss sich die Spezifität der identifizierten zweidimensionalen Struktur auch in hypothesengeleiteten differenziellen Bedingungsanalysen bewähren (Stufe II: spezifische Validierung). Im Rahmen der spezifischen Validierung muss beispielsweise gezeigt werden, dass die identifizierten Kompetenzfacetten differenziell mit Außenkriterien assoziiert sind oder unterschiedliche Entwicklungsverläufe aufweisen. Die Prüfung der spezifischen Validität erfolgte im LUK-Projekt auf Grundlage theoretischer Modelle, anhand derer in verschiedenen Einzelstudien untersucht wurde, ob Facetten motivationaler und affektiver Lernermerkmale differenziell mit textartspezifischen Verstehensleistungen (literarisch vs. faktual) assoziiert sind (vgl. Henschel 2013). Hierbei zeigte sich, dass einzelne Facetten der Empathie (Fantasieempathie, vgl. Henschel/Roick 2013), der intrinsischen Lesemotivation (erlebnisbezogene Lesemotivation, vgl. Henschel/Schaffner 2014) und des Leseselbstkonzeptes (literarisches Leseselbstkonzept, vgl. Henschel et al. 2013) signifikant stärker mit dem Verstehen literarischer als faktualer Texte zusammenhingen. Diese Studien liefern damit weitere Belege für die angenommene Zweidimensionalität des Textverstehens. Könnten die identifizierten Merkmale auch in längsschnittlich angelegten Studien insbesondere zur Vorhersage des literarischen 
Textverstehens (im Vergleich zum faktualen Textverstehen) beitragen, sollte ihre Ausbildung und Entwicklung z. B. im Literaturunterricht besonders unterstützt werden. Inwiefern die Merkmale, die in den Einzelstudien identifiziert wurden, in einer längsschnittlich angelegten Modellierung tatsächlich zur Erklärung des literarischen Textverstehens beitragen, wurde im Rahmen der nomologischen Validierung (Stufe III, vgl. Henschel 2013, Henschel/Roick 2013) untersucht. Das Ziel der nomologischen Validierung besteht vor allem darin, die Bedeutung von Kompetenzstrukturmodellen bzw. einzelnen Facetten der Kompetenzstruktur (hier des literarischen Textverstehens) für schulische Lehr- und Lernprozesse dahingehend zu prüfen, inwiefern sie sich für die Theorieentwicklung und Theorieerweiterung bewähren (vgl. Roick/Henschel 2015). Dazu wurden die drei affektiven und motivationalen Merkmale (Fantasieempathie, literarisches Leseselbstkonzept und erlebnisbezogene Lesemotivation) in eine vorhandene theoretische (Erwartungsmal Wert-) Modellvorstellung (Möller/Schiefele 2004) integriert (vgl. Henschel 2013). Die längsschnittliche Modellierung wies auf differenzielle Mediationsprozesse hin. Diese legen nahe, dass sowohl eher affektive als auch eher kognitive Erklärungsmechanismen beim Verstehen literarischer Texte bedeutsam zu sein scheinen, die unabhängig voneinander Varianz im literarischen Textverstehen erklären. So zeigte sich, dass bei einer längsschnittlichen Betrachtung kein direkter Effekt der Fantasieempathie auf das literarische Textverstehen zu beobachten ist, sondern dieser vor allem über die auf stellvertretendes Erleben bzw. Flow-Erleben ausgerichtete erlebnisbezogene Lesemotivation transportiert wurde (affektiver Vermittlungsmechanismus). Auch das literarische Leseselbstkonzept, welches die kognitiv-evaluative Fähigkeitsselbsteinschätzung bezüglich des Verstehens literarischer Texte beschreibt, wirkte sich in der längsschnittlichen Betrachtung nicht direkt, sondern ausschließlich indirekt auf das literarische Textverstehen aus. Im Gegensatz zur Fantasieempathie transportiert hier aber nicht die erlebnisbezogene Lesemotivation den Effekt auf das literarische Textverstehen, sondern die auf das thematische Interesse ausgerichtete gegenstandsbezogene Lesemotivation. Hierbei handelte es sich also um einen eher kognitiven Vermittlungsmechanismus.

Insgesamt liefern die durchgeführten Studien umfangreiche empirische Hinweise darauf, dass das Textverstehen als zweidimensionales (literarisch vs. faktuales) Konstrukt beschrieben werden kann und das literarische Textverstehen durch spezifische affektive (Fantasieempathie) und motivationale Lernermerkmale (literarisches Leseselbstkonzept, erlebnisbezogene Lesemotivation) erklärbar ist, die kaum oder gar nicht zur Erklärung des faktualen Textverstehens beitragen. Erst auf Grundlage dieses umfassenden Validierungsprozesses, der die angenommene zweidimensionale Kompetenzstruktur des Textverstehens stützt, ist eine genauere 
Untersuchung der internen Struktur des literarischen Textverstehens, die mit der zweiten Forschungsfrage in diesem Beitrag aufgegriffen wurde, sinnvoll. Zwar weisen die ersten strukturellen Analysen, die im LUK Projekt durchgeführt wurden und ebenfalls in Abschnitt drei angesprochen wurden, auch darauf hin, dass eine Unterscheidung zwischen den beiden Kerndimensionen des semantischen und idiolektalen Verstehens angemessen sein könnte (vgl. Frederking et al. 2012a). Allerdings zeigte sich auch, dass der Zusammenhang zwischen diesen beiden Dimensionen offenbar in Abhängigkeit vom Kompetenzniveau steigt und beispielsweise in der Gruppe schwächerer Lerner an Hauptschulen am niedrigsten ausfiel, d. h. dort waren beide Kompetenzfacetten am besten unterscheidbar (vgl. Roick et al. 2013). Ein Grund könnte darin liegen, dass die Leistungsdiskrepanz zwischen dem semantischen und idiolektalen Textverstehen bei schwächeren Lernern (z. B. an Hauptschulen) signifikant größer zu sein scheint als bei leistungsstärkeren Jugendlichen. Letztere weisen zwar ebenfalls signifikant schwächere Leistungen im idiolektalen Verstehen im Vergleich zum semantischen Verstehen auf, diese Diskrepanz ist aber deutlich geringer ausgeprägt als bei schwächeren Lernenden. Daraus lässt sich die Annahme ableiten, dass beide Kompetenzfacetten nicht gleichermaßen ausgebildet werden bzw. spezifische (Förder-)Bedingungen die Ausbildung beider Verstehensbereiche differenziell begünstigen. Sofern in längsschnittlichen Analysen oder im Rahmen experimenteller Studien differenzielle Entwicklungsverläufe beider Verstehensdimensionen beobachtet werden können, würde dies die strukturellen Analysebefunde zur Abgrenzbarkeit des semantischen und idiolektalen Verstehens weiter stützen. Zu berücksichtigen ist aber auch, dass das Semantische und Idiolektale das literarische Textverstehen noch nicht hinreichend beschreiben, sondern weitere Facetten berücksichtigt werden müssen. Erste Analysen bei leistungsstarken Jugendlichen an Gymnasien deuten an, dass bei der Beschreibung der Kompetenzstruktur des literarischen Textverstehens auch Aspekte berücksichtigt werden sollten, die etwa die Anwendung literarischen Fachwissens, die Fähigkeit, ästhetisch bedeutsame Auffälligkeiten im Text zu entdecken, oder die textseitig intendierten Emotionen erkennen zu können, betreffen (vgl. Meier et al., im Druck).

\section{Interdisziplinäre Forschung zwischen Literaturdidaktik und empirischer Bildungsforschung (Volker Frederking/Sofie Henschel)}

Das LUK-Projekt ist sicherlich ein sehr gutes Beispiel für die Fruchtbarkeit interdisziplinärer Zusammenarbeit im Bereich der fach- bzw. domänenspezifischen Bildungsforschung. Tatsächlich konnten die in LUK zu klärenden Forschungsfragen jeweils nur im interdisziplinären Diskurs und in fruchtbarer interdisziplinärer 
Zusammenarbeit gelöst werden. Die beiden in den Abschnitten drei und vier exemplarisch angeführten und erläuterten Forschungsschwerpunkte im Rahmen des LUK-Projekts haben gleichzeitig ins Blickfeld treten lassen, dass im interdisziplinären Zusammenwirken zweier Disziplinen wie der Literaturdidaktik und der empirischen Bildungswissenschaft auch unterschiedliche Schwerpunkte zutage treten bzw. gesetzt werden müssen. Diese erklären sich aus disziplinären Besonderheiten und Traditionen und sind Chance und Herausforderung zugleich. Nachfolgend sollen Erfahrungen und Schlussfolgerungen formuliert und zur Diskussion gestellt werden.

\subsection{Interdisziplinäre Forschung im LUK-Projekt aus erziehungswissenschaftlich-psychologischer Sicht (Sofie Henschel)}

\section{Gelingensbedingungen interdisziplinärer Forschung}

Gelingensbedingungen interdisziplinärer Zusammenarbeit sind auch Gelingensbedingungen der empirischen Bildungsforschung, deren zentrale Bezugsdisziplinen die Erziehungswissenschaft, die Fachdidaktiken und die pädagogische Psychologie sind (vgl. Prenzel 2006). Der Erfolg der empirischen Bildungsforschung setzt die Entwicklung integrativer Forschungsansätze voraus, die sich am internationalen Wissensstand orientieren und die Anwendung vielfältiger empirischer Forschungsmethoden einschließen. Interdisziplinäre Zusammenarbeit sollte durch Problemorientierung und gegenseitige Wertschätzung der spezifischen Kompetenzen der beteiligten Disziplinen gekennzeichnet sein, die eine eigene Forschungsgeschichte mit Bezug auf den Forschungsgegenstand aufweisen. Es müssen deshalb auch Interessen der anderen Disziplinen akzeptiert werden können, die Interessen der eigenen Disziplin möglicherweise entgegenstehen. Wichtig ist in diesem Zusammenhang dennoch, der eigenen Disziplin und deren Qualitätsstandards treu zu bleiben. So sollten etwa die Interpretation von Befunden und die Reflexion von Theorien im wissenschaftlichen Horizont der eigenen Disziplin erfolgen. Dabei kann die Weiterentwicklung von Theorien in einem interdisziplinären Diskurs eine besondere Herausforderung darstellen, wenn empirische Forschung von einer der beteiligten Disziplinen vor allem als ,Heilsbringer' zur Zementierung vorhandener Theorien betrachtet wird. Interdisziplinäre Forschung kann nur erfolgreich sein, wenn theoretische Annahmen auf Basis empirischer Befunde „an der Wirklichkeit scheitern“ dürfen (Prenzel 2006, S. 73). 


\section{Chancen und Schwierigkeiten interdisziplinärer Forschung}

Die interdisziplinäre Zusammenarbeit mit der Deutsch- und Literaturdidaktik erweist sich für die Erziehungswissenschaft und die pädagogische Psychologie vor allem für die Ausdifferenzierung vorhandener Modellvorstellungen als wichtiger Impulsgeber. Das betrifft vor allem die Notwendigkeit einer stärkeren Integration emotionaler Merkmale und Prozesse in Erklärungsmodelle des Textverstehens auf Ebene des Lernens und des Lehrens.

Grenzen und Schwierigkeiten bei der fächerübergreifenden Bearbeitung deutschdidaktischer Fragestellungen ergeben sich aus den Wurzeln der beteiligten Disziplinen. So liegt der Fokus deutschdidaktischer Forschung häufig eher auf dem fachinternen nationalen Diskurs statt auf internationaler Anschlussfähigkeit, scheint die Theoriewahrung oftmals der Theorieentwicklung vorgezogen zu werden und werden theoretische Modelle eher vor dem Hintergrund der Vollständigkeit statt der Einfachheit und Nützlichkeit bewertet.

\subsection{Interdisziplinäre Forschung im LUK-Projekt aus literaturdidaktischer Sicht (Volker Frederking)}

\section{Gelingensbedingungen interdisziplinärer Forschung}

Eine wesentliche Voraussetzung für das Gelingen einer interdisziplinären Forschungskooperation ist eine, gute Mischung' aus wissenschaftlicher Neugier und gegenseitigem Respekt vor den disziplinären Besonderheiten des Kooperationspartners. Interdisziplinäres Zusammenarbeiten ähnelt in gewisser Hinsicht dem Erlernen einer Fremdsprache - das dauert und es ist klar, dass man in dieser ,Fremdsprache` selten so gut sein wird wie ein disziplinärer ,Muttersprachler.

Disziplinäre Herausforderungen interdisziplinärer Forschung Interdisziplinäre Forschung wird erschwert, wenn sie in der eigenen Disziplin argwöhnisch beäugt und als „Häresie“ (Brüggemann 2013) diskreditiert wird. Tatsächlich gibt es in der Deutschdidaktik - anders als in der empirischen Bildungswissenschaft - leider eine Tendenz, ideologisch zu polemisieren statt rational und sachlich zu argumentieren. Das verfehlt die Standards eines wissenschaftlichen Diskurses innerhalb einer Disziplin und erschwert den interdisziplinären Diskurs mit anderen Disziplinen. Mittelfristig könnte dadurch auch der Status der Deutschdidaktik als Wissenschaft Schaden nehmen.

\section{Schwierigkeiten interdisziplinärer Forschung}

Interdisziplinäre Forschung benötigt immer ein gewisses Maß an akademischer Ambiguitätstoleranz. Im Zusammenhang mit der Pädagogischen Psychologie 
stellt die aus ihrer Geschichte resultierende Skepsis gegenüber hermeneutisch motivierter theoretischer Differenzierung und Komplexität sicherlich eine spezifische Schwierigkeit dar. So traf die Idee einer Komplexitätserweiterung des Modells im Zusammenhang mit emotionalen Facetten literarischer Verstehenskompetenz zunächst auf große Skepsis bei den psychologischen Kooperationspartnern. Dabei erwies sich das disziplinspezifische Favorisieren der einfacheren Lösung als schwer zu überwindender Widerstand. Dass dies letztendlich dennoch möglich war (vgl. Frederking et al. 2015), spricht für die Fruchtbarkeit der interdisziplinären Kooperation.

\section{Chancen interdisziplinärer Forschung}

Literaturdidaktik und Deutschdidaktik können von der interdisziplinären $\mathrm{Zu}$ sammenarbeit mit der Pädagogischen Psychologie und empirischen Erziehungswissenschaft nur profitieren. Denn auf diese Weise erweitern sich theoretisches Wissen und methodisches Spektrum gleichermaßen. Literaturdidaktik und Deutschdidaktik können sich mit anderen Worten - wie viele andere Fachdidaktiken zuvor - zu empirisch forschenden Disziplinen entwickeln, die dem Prinzip der Evidenzorientierung verpflichtet sind, ohne dabei ihre hermeneutischen Wurzeln zu suspendieren.

\section{Literatur}

Albrecht, Christian/Hornberger, Johannes (2014): Empirische Zugänge zu ästhetischer Erfahrung. In: Jahraus, Oliver/Liebau, Eckart/Pöppel, Ernst/Wagner, Ernst (Hrsg.): Gestalten und Erkennen. Ästhetische Bildung und Kompetenz. Münster/New York: Waxmann, S. 49-61.

Altmann, Ulrike/Bohrn, Isabel C./Lubrich, Oliver/Menninghaus, Winfried/ Jacobs, Arthur M. (2014): Fact vs. fiction - how paratextual information shapes our reading processes. In: Social Cognitive and Affective Neuroscience. Vol. 9. No. 1, pp. 22-29.

Artelt, Cordula/Schlagmüller, Matthias (2004): Der Umgang mit literarischen Texten als Teilkompetenz im Lesen? Dimensionsanalysen und Ländervergleiche. In: Schiefele, Ulrich/Artelt, Cordula/Schneider, Wolfgang/Stanat, Petra (Hrsg.), Struktur, Entwicklung und Förderung von Lesekompetenz: Vertiefende Analysen im Rahmen von PISA 2000. Wiesbaden: Verlag für Sozialwissenschaften, S. 169-196.

Baum, Michael (2013): Literarisches Verstehen und Nichtverstehen. In: Frederking, Volker/Krommer, Axel /Meier, Christel (Hrsg.): Literatur- und Mediendidaktik. Taschenbuch des Deutschunterrichts. Band 2. 2., aktual. Aufl. Baltmannsweiler: Schneider, S. 102-125. 
Bertschi-Kaufmann, Andrea/Rosebrock, Cornelia (Hrsg.) (2009): Literalität: Bildungsaufgabe und Forschungsfeld. Weinheim/München: Juventa.

Bertschi-Kaufmann, Andrea/Rosebrock, Cornelia (Hrsg.) (2013): Literalität erfassen: bildungspolitisch, kulturell, individuell. Weinheim/Basel: Beltz Juventa.

Böhme, Katrin/Robitzsch, Alexander (2009): Methodische Aspekte der Erfassung der Lesekompetenz. In: Granzer, Dietlinde/Köller, Olaf/Bremerich-Vos, Albert/Heuvel-Panhuizen, Marja van den/Reiss, Kristina/Walther, Gerd (Hrsg.): Bildungsstandards Deutsch und Mathematik. Weinheim: Beltz, S. 259-298.

Bourg, Tammy (1996): The role of emotion, empathy and text structure in children's and adults' narrative text comprehension. In: Kreuz, Roger J./MacNealy, Mary Sue (Eds.): Empirical approaches to literature and aesthetics. Westport, CT: Ablex Publishing, pp. 241-260.

Brüggemann, Jörn (2013): Deutschdidaktik und Germanistik. Analyse einer umstrittenen Beziehung. In: Frederking, Volker/Krommer, Axel (Hrsg.): Aktuelle Fragen der Deutschdidaktik. Taschenbuch des Deutschunterrichts. Band 3. Baltmannsweiler: Schneider, S. 143-176.

Brüggemann, Jörn/Frederking, Volker (2015): Literarische Interpretations- und Kommunikationskulturen im Blick empirischer Forschung. In: Lessing-Sattari, Marie/Wieser, Dorothee/Löhden, Maike/Meissner, Almuth (Hrsg.): Interpretationskulturen: Literaturdidaktik und Literaturwissenschaft im Dialog über Theorie und Praxis des Interpretierens. Frankfurt a. M.: Lang, S. 219-242.

Bühler, Axel (2003): Grundprobleme der Hermeneutik. In: Ders. (Hrsg.): Hermeneutik. Basistexte zur Einführung in die wissenschaftstheoretischen Grundlagen von Verstehen und Interpretation. Heidelberg: Synchron, S. 3-19.

Currie, Gregory (1990): The nature of fiction. New York u. a.: Cambridge University Press.

Drewes, Jana/Albrecht, Christian (2014): ÄSKIL. Ein Projekt zur empirischen Erforschung literarischer Gespräche im Deutschunterricht. In: Liebau, Eckart/Jörissen, Benjamin/Klepacki, Leopold (Hrsg.): Forschung zur Kulturellen Bildung. Grundlagenreflexionen und empirische Befunde. München: kopaed, S. $101-107$.

De Man, Paul (1983) [1971]: Blindness and Insight. Essays in the Rhetoric of Contemporary Criticism. Second Edition, Revised. Introduction by Wlad Godzich. Minneapolis: University Of Minnesota Press.

Eco, Umberto (1996): Zwischen Autor und Text. Interpretation und Überinterpretation. München: Deutscher Taschenbuch Verlag.

Eco, Umberto (1998a) [1962]: Das offene Kunstwerk. Frankfurt a. M.: Suhrkamp.

Eco, Umberto (1998b): Lector in fabula. Die Mitarbeit der Interpretation in erzählenden Texten. München: Deutscher Taschenbuch Verlag. 
Eco, Umberto (1999): Die Grenzen der Interpretation. München: Deutscher Taschenbuch Verlag.

Eco, Umberto (2002) [1972]: Einführung in die Semiotik. München: Fink.

Eco, Umberto (2006): Quasi dasselbe mit anderen Worten. Über das Übersetzen. München: Deutscher Taschenbuch Verlag.

Eva-Wood, Amy L. (2004): Thinking and feeling poetry: Exploring meanings aloud. In: Journal of Educational Psychology. Vol. 96. No. 1, pp. 182-191.

Fialho, Olivia (2007): Foregrounding and refamiliarization: understanding readers' response to literary texts. In: Language and Literature. Vol. 16. No. 2, pp. 105-123.

Frederking, Volker (2003): „Es werde von Grund aus anders“!? Leseinteresse, Lernmotivation und Selbstregulation im Deutschunterricht nach PISA und IGLU. In: Abraham, Ulf/Bremerich-Vos, Albert/Frederking, Volker/Wieler, Petra (Hrsg.): Deutschunterricht und Deutschdidaktik nach PISA. Freiburg i. Br.: Fillibach, S. 249-278.

Frederking, Volker/Meier, Christel/Stanat, Petra/Dickhäuser, Oliver (2008): Ein Modell literarästhetischer Urteilskompetenz. In: Didaktik Deutsch. Jg. 13. H. 25, S. 11-31.

Frederking, Volker/Roick, Thorsten/Steinhauer, Lydia (2011): Literarästhetische Urteilskompetenz. Forschungsansatz und Zwischenergebnisse. In: Bayrhuber, Horst/Harms, Ute/Muszynski, Bernhard/Ralle, Bernd/Rothgangel, Martin/ Schön, Lutz-Helmut/Vollmer, Helmut J./Weigand, Hans-Georg (Hrsg.): Empirische Fundierung in den Fachdidaktiken. Münster: Waxmann, S. 75-94.

Frederking, Volker/Meier, Christel/Brüggemann, Jörn/Gerner, Volker/Friederich, Marcus (2011): Literarästhetische Verstehenskompetenz - theoretische Modellierung und empirische Erforschung: In: Zeitschrift für Germanistik. Jg. 11. H. 1, S. 131-144.

Frederking, Volker/Henschel, Sofie/Meier, Christel/Roick, Thorsten/Stanat, Petra/ Dickhäuser, Oliver (2012a): Beyond functional aspects of reading literacy: Theoretical structure and empirical validity of literary literacy. In: L1 Educational Studies in Language and Literature. Vol. 12, pp. 1-24.

Frederking, Volker/Gerner, Volker/Brüggemann, Jörn/Albrecht, Christian/Henschel, Sofie/Roick, Thorsten/Meier, Christel/Rieder, Adelheid (2012b): Literarästhetische Kommunikation im Deutschunterricht: In: Becker-Mrotzek, Michael/Schramm, Karen/Thürmann, Eike/Vollmer, Helmut Johannes (Hrsg.): Sprache im Fach. Sprachlichkeit und fachliches Lernen. Münster/New York: Waxmann, S. 131-147. 
Frederking, Volker (2014): Deutschdidaktik als transdisziplinäre, anwendungsund grundlagenorientierte empirische Wissenschaft. Mitteilungen des Deutschen Germanistenverbandes. Jg. 61. H. 2, S. 109-119.

Frederking, Volker/Brüggemann, Jörn/Albrecht, Christian/Henschel, Sofie/Gölitz, Dietmar (2015): Emotionale Facetten literarischen Verstehens und ästhetischer Erfahrung. Empirische Befunde literaturdidaktischer Grundlagen- und Anwendungsforschung. In: Brüggemann, Jörn/Dehrmann, Mark-Georg/Standke, Jan (Hrsg.): Literarizität. Herausforderungen für Theoriebildung, empirische Forschung und Vermittlung. Fachdidaktische und literaturwissenschaftliche Perspektiven. Baltmannsweiler: Schneider, S. 87-132.

Frederking, Volker/Wieser, Dorothee (2015): Sich mit Texten und Medien auseinandersetzen. In: Becker-Mrotzek, Michael/Kämper-van den Boogaart, Michael/ Köster, Juliane/Stanat, Petra/Gippner, Gabriele (Hrsg.): Bildungsstandards aktuell: Deutsch in der Sekundarstufe II. Braunschweig: Bildungshaus Schulbuchverlage, S. 176-235.

Fricke, Harald. (1991): Literatur und Literaturwissenschaft. Beiträge zu Grundfragen einer verunsicherten Disziplin. Paderborn u. a.: Schöningh.

Genette, Gérard (2001) [1987]: Paratexte. Das Buch vom Beiwerk des Buches. Frankfurt a. M.: Suhrkamp.

Groeben, Norbert (2002): Zur konzeptionellen Struktur des Konstrukts „Lesekompetenz“. In: Groeben, Norbert/Hurrelmann, Bettina (Hrsg.): Lesekompetenz. Bedingungen, Dimensionen, Funktionen. Weinheim/München: Juventa, S. 11-20.

Grünkorn, Juliane/Fleischer Jens/Klieme, Eckhard (2014): Symposium Kompetenzveränderung im Längsschnitt. In: GEBF 2014. Die Perspektiven verbinden. 2. Tagung in Frankfurt am Main. 3. bis 5. März 2014. Goethe-Universität.

Härle, Gerhard (2011): „und am Schluss weiß ich trotzdem nicht, was der Text sagt“. Grundlagen, Zielperspektiven und Methoden des Literarischen Unterrichtsgesprächs. In: Steinbrenner, Markus/Mayer, Johannes/Rank, Bernhard (Hrsg.): „Seit ein Gespräch wir sind und hören voneinander“. Das Heidelberger Modell des Literarischen Unterrichtsgesprächs in Theorie und Praxis. Baltmannsweiler: Schneider, S. 29-65.

Hartig, Johannes/Frey, Andreas/Jude, Nina (2007): Validität. In: Moosbrugger, Helfried/Kelava, Augustin (Hrsg.): Testtheorie und Fragebogenkonstruktion. Berlin/Heidelberg: Springer, S. 135-163.

Heckhausen, Heinz (1987): „Interdisziplinäre Forschung“ zwischen Intra-, Multiund Chimären-Disziplinarität. In: Kocka, Jürgen (Hrsg.): Interdisziplinarität. Praxis - Herausforderung - Ideologie. Frankfurt a. M.: Suhrkamp, S. 129-145. 
Henschel, Sofie (2013): Effekte motivationaler und affektiver Merkmale auf das Verstehen literarischer und faktualer Texte. Berlin: Freie Universität Berlin.

Henschel, Sofie/Roick, Thorsten (2013): Zusammenhang zwischen Empathie und dem Verstehen literarischer Texte. In: Zeitschrift für Entwicklungspsychologie und Pädagogische Psychologie. Jg. 45. H. 2, S. 103-113.

Henschel, Sofie/Roick, Thorsten/Brunner, Martin/Stanat, Petra (2013): Leseselbstkonzept und Textart: Lassen sich literarisches und faktuales Leseselbstkonzept trennen? In: Zeitschrift für Pädagogische Psychologie. Jg. 27. H. 3, S. 181-191.

Henschel, Sofie/Schaffner, Ellen (2014): Differenzielle Zusammenhänge zwischen Komponenten der Lesemotivation und dem Verständnis literarischer bzw. expositorischer Texte. In: Psychologie in Erziehung und Unterricht. Jg. 61. H. 2, S. $112-126$.

Hirsch, Eric D. (1967): Validity in Interpretation. New Haven/London: Yale University Press.

Hirsch, Matthias (2014): Die Kompetenz historischen Lesens. Theoretische Konturierung und empirische Annäherung. Masterarbeit an der Katholischen Universität Eichstätt-Ingolstadt.

Jannidis, Fotis/Lauer, Gerhard/Martinez, Matias/Winko, Simone (1999): Rückkehr des Autors. Tübingen: Niemeyer.

Jannidis, Fotis/Gerhard Lauer/Matlas Martinez/Simone Winko (Hrsg.) (2003): Regeln der Bedeutung. Zur Theorie der Bedeutung literarischer Texte. Berlin/ New York: de Gruyter.

Kaufmann, Franz-Xaver (1987): Interdisziplinäre Wissenschaftspraxis. Erfahrungen und Kriterien. In: Kocka, Jürgen (Hrsg.): Interdisziplinarität. Praxis Herausforderung - Ideologie. Frankfurt a. M., S. 63-81.

Kammler, Clemens (2006): Vorwort des Herausgebers. In: Kammler, Clemens (Hrsg.): Literarische Kompetenzen - Standards im Literaturunterricht. Modelle für die Primar- und Sekundarstufe. Seelze: Kallmeyer/Klett, S. 5-6.

Kintsch, Walter (1994): Kognitionspsychologische Modelle des Textverstehens: Literarische Texte. In: Reusser, Kurt/Reusser-Weyeneth; Marianne (Hrsg.): Verstehen. Psychologischer Prozeß und didaktische Aufgabe. Bern u. a.: Hans Huber, S. 39-54.

Kintsch, Walter (2000): Metaphor comprehension: A computational theory. Psychonomic Bulletin \& Review. Vol. 7. No. 2, pp. 257-266.

KMK (2003): Bildungsstandards im Fach Deutsch für den Mittleren Schulabschluss. Beschluss vom 4.12.2003. München: Luchterhand.

KMK (2004): Beschlüsse der Kultusministerkonferenz. Bildungsstandards im Fach Deutsch für den Hauptschulabschluss. Beschluss vom 15.10.2004. München: Luchterhand. 
KMK (2012) Bildungsstandards im Fach Deutsch für die Allgemeine Hochschulreife (Beschluss der Kultusministerkonferenz vom 18.10.2012). http://www.kmk. org/fileadmin/Dateien/veroeffentlichungen_beschluesse/2012/2012_10_18Bildungsstandards-Deutsch-Abi.pdf. Abgerufen am 23.02.2016.

Kristeva, Julia (1972): Bachtin, das Wort, der Dialog und der Roman. In: Ihwe, Jens (Hrsg.): Literaturwissenschaft und Linguistik. Ergebnisse und Perspektiven. Bd. 3: Zur linguistischen Basis der Literaturwissenschaft II. Frankfurt a. M.: Athenäum, S. 345-375.

Krommer, Axel (2003): Das Verstehen literarischen Verstehens als interdisziplinäres Projekt. Anmerkungen zur Kognitionspsychologie Walter Kintschs aus deutschdidaktischer Sicht. In: Abraham, Ulf/Bremerich-Vos, Albert/Frederking, Volker/Wieler, Petra (Hrsg.): Deutschunterricht und Deutschdidaktik nach PISA und IGLU. Freiburg: Fillibach, S. 165-187.

Lüdtke, Jana/Jacobs, Arthur/Meyer-Sickendiek, Burkhard (2013): Foregrounding/ Backgrounding: Bausteine einer neurokognitiven Poetik. In: Reents, Friederike/Meyer-Sickendiek, Burkhard (Hrsg.): Stimmung und Methode. Tübingen: Mohr Siebeck, S. 63-94.

Luhmann, Niklas (1992): Die Wissenschaft der Gesellschaft. Frankfurt a. M.: Suhrkamp.

Luhmann, Niklas (1995): Soziale Systeme. Frankfurt a. M.: Suhrkamp.

McNamara, Danielle S./Magliano, Joe (2009): Toward a comprehensive model of comprehension. In: Ross, Brian H. (Ed.): The psychology of learning and motivation. Vol. 51. San Diego, CA, US: Elsevier Academic Press, pp. 297-384.

Meier, Christel/Roick, Thorsten/Henschel, Sofie/Brüggemann, Jörn/Frederking, Volker/Rieder, Adelheid/Gerner, Volker/Stanat, Petra (in press): An extended model of literary literacy. In: Leutner, Detlef/Fleischer, Jens/Grünkorn, Juliane/Klieme, Eckard (Eds.): Competence assessment in education: Research, models, and instruments. New York: Springer.

Meutsch, Dietrich (1987): Literatur verstehen. Eine empirische Studie. Braunschweig: Friedrich Vieweg \& Sohn.

Mittelstraß, Jürgen (1987): Die Stunde der Interdisziplinarität? In: Kocka, Jürgen (Hrsg.): Interdisziplinarität. Praxis - Herausforderung - Ideologie. Frankfurt a. M.: Suhrkamp, S. 152-158.

Mittelstraß, Jürgen (1992): Leonardo-Welt. Über Wissenschaft, Forschung und Verantwortung. Frankfurt a. M.: Suhrkamp.

Müller, Hans-Harald (2000): Eco zwischen Autor und Text. Eine Kritik von Ecos Interpretationstheorie. In: Kindt, Tom/Müller, Hans-Harald (Hrsg.): Ecos Echos. Das Werk Umberto Ecos: Dimensionen, Rezeptionen, Kritiken. München: Fink, S. 135-148. 
Möller, Jens/Schiefele, Ulrich (2004): Motivationale Grundlagen der Lesekompetenz. In: Schiefele, Ulrich/Artelt, Cordula/Schneider, Wolfgang/Stanat, Petra (Hrsg.): Struktur, Entwicklung und Förderung von Lesekompetenz. Vertiefende Analysen im Rahmen von PISA 2000. Wiesbaden: Verlag für Sozialwissenschaften, S. 101-124.

Pant, Hans Anand (2014): Aufbereitung von Evidenz für bildungspolitische und pädagogische Entscheidungen: Metaanalysen in der Bildungsforschung. In: Bundesministerium für Bildung und Forschung (BMBF) (Hrsg.): Bildungsforschung 2020. Herausforderungen und Perspektiven. Bonn/Berlin, S. 147-170.

Popper, Karl Raimund (2005) [1935]: Logik der Forschung. Tübingen: Mohr Siebeck.

Popper, Karl Raimund (1992) [1972]: Objektive Erkenntnis. Ein evolutionärer Entwurf. Hamburg: Hoffmann \& Campe.

Prediger, Susanne (2014): Unterrichtsqualität braucht Fachlichkeit - Fachdidaktische Forschung und Entwicklung als Bindeglied zwischen Grundlagenforschung und Unterrichtspraxis. Forum auf der Tagung Bildungsforschung 2020. Zwischen wissenschaftlicher Exzellenz und gesellschafticher Verantwortung. 27./28.03.2014. Berlin: BMBF.

Prenzel, Manfred (2006): Bildungsforschung zwischen Pädagogischer Psychologie und Erziehungswissenschaft. In: Merkens, Hans (Hrsg.): Erziehungswissenschaft und Bildungsforschung. Wiesbaden: VS Verlag für Sozialwissenschaften, S. 69-79.

Roick, Thorsten/Stanat, Petra/Dickhäuser Oliver/Frederking, Volker/Meier, Christel/Steinhauer, Lydia. (2010): Projekt Literarästhetische Urteilskompetenz. Strukturelle und kriteriale Validität der literarästhetischen Urteilskompetenz. In: Klieme, Eckhart/Leutner, Detlev/Kenk, Martina (Hrsg.): Kompetenzmodellierung. Zwischenbericht des DFG-Schwerpunktprogramms und Perspektiven des Forschungsansatzes. Zeitschrift für Pädagogik. 56. Beiheft. Weinheim/ Basel: Beltz, S. 165-174.

Roick, Thorsten/Frederking, Volker/Henschel, Sofie/Meier, Christel (2013): Literarische Textverstehenskompetenz bei Schülerinnen und Schülern unterschiedlicher Schulformen. In: Bertschi-Kaufmann, Andrea/Rosebrock, Cornelia (Hrsg.): Literalität erfassen: bildungspolitisch, kulturell, individuell. Weinheim: Juventa, S. 69-84.

Roick, Thorsten/Henschel, Sofie (2015): Strategie zur Validierung von Kompetenzstrukturmodellen. In: Riegel, Ulrich/Schubert, Sigrid/Siebert-Ott, Gesa/ Macha, Klaas (Hrsg.): Kompetenzmodellierung und Kompetenzmessung in den Fachdidaktiken. Münster: Waxmann, S. 11-28.

Rolle, Christian (2011): Ästhetische Bildung durch Kompetenzerwerb? Über Probleme mit Standards und Messverfahren in den künstlerischen Fächern 
am Beispiel der Musik. http://www.uni-saarland.de/fileadmin/user_upload/ Einrichtungen/zfl/PDF_Fachdidaktik/C.Rolle_aesthetische_Bildung_durch_ Kompetenzerwerb.pdf. Abgerufen am 23.02.2016.

Rosenberg, Rainer (1992): Epochen. In: Brackert, Helmut /Stückrath, Jörn (Hrsg.): Literaturwissenschaft. Ein Grundkurs. Reinbek bei Hamburg: Rowohlt, S. 269-280.

Stierle, Karlheinz (1984): Werk und Intertextualität. In: Stierle, Karlheinz/Warning, Rainer (Hrsg.): Das Gespräch. Poetik und Hermeneutik XI. München: Fink, S. 139-150.

Trautwein, Ulrich (2014): Symposium „Literarästhetische und Historische Kompetenzen: Kompetenzmodelle, Erfassung und Validierung“. In: GEBF 2014. Die Perspektiven verbinden. 2. Tagung in Frankfurt am Main. 3. bis 5. März 2014. Goethe-Universität.

Voßkamp, Wilhelm (1992): Gattungen. In: Brackert, Helmut/Stückrath, Jörn (Hrsg.): Literaturwissenschaft. Ein Grundkurs. Reinbek b. Hamburg: Rowohlt, S. 253-269.

Wermke, Jutta (1989): „Hab a Talent, sei a Genie!“. Kreativität als paradoxe Aufgabe. Band 1: Entwicklung eines Konzepts der Kreativität und ihrer Förderung durch Literatur. Band 2: Empirische Überprüfung literaturdidaktischer Möglichkeiten der Kreativitätsförderung. Weinheim: Beltz.

Zabka, Thomas (2006): Typische Operationen literarischen Verstehens. Zu Martin Luther „Vom Raben und Fuchs“ (5./6. Schuljahr). In: Kammler, Clemens (Hrsg.): Literarische Kompetenzen - Standards im Literaturunterricht. Modelle für die Primar- und Sekundarstufe. Seelze: Kallmeyer/Klett, S. 80-101.

Zwaan, Rolf A. (1993): Aspects of literary comprehension: A cognitive approach. Amsterdam: John Benjamins. 
Iris Winkler and Frederike Schmidt - 978-3-631-69285-1

Downloaded from PubFactory at 01/11/2019 10:32:53AM

via free access 
Katrin Böhme, Petra Stanat, Michael Becker-Mrotzek

\title{
Die Entwicklung integrierter Kompetenzstufenmodelle als Ergebnis interdisziplinärer Kooperation
}

\begin{abstract}
Models of competence levels constitute a central element of national educational monitoring. The development of such models requires a close interdisciplinary cooperation between experts from subject-matter education and empirical educational research. In this article, we aim to provide an example for this cooperation by describing the development of integrated proficiency level models for German at secondary level I.
\end{abstract}

\section{Einführung}

Die Kultusministerkonferenz (KMK) leitete zu Beginn des neuen Jahrtausends einen weitreichenden Reformprozess in der deutschen Bildungspolitik ein. Die intendierte Umgestaltung basierte auf einem Paradigmenwechsel, der die lange Zeit dominierende Input- und Prozessorientierung durch Elemente einer intensivierten Outputorientierung ergänzte. Damit erhielt die Frage danach, welche Lernergebnisse im Unterricht erreicht werden, einen prominenteren Stellenwert. Die datengestützte Evaluation von Bildungsergebnissen hat somit an Bedeutung gewonnen und die Steuerung des deutschen Bildungssystems basiert seitdem verstärkt auf empirisch erhobenen Daten. Die Entwicklung und Sicherung der Bildungsqualität orientiert sich nunmehr stärker an den Bildungserträgen der Schülerinnen und Schüler, der Schulen sowie des schulischen Bildungssystems insgesamt (Böhme et al. 2012, S. 11).

Um für alle Länder in Deutschland eine gemeinsame Grundlage der Qualitätsentwicklung und des Bildungsmonitorings zu schaffen, erteilte die Kultusministerkonferenz zu Beginn des Jahrtausends den Auftrag, länderübergreifende Bildungsstandards für bestimmte Kernfächer und Schulabschlüsse zu entwickeln. Diese wurden für den Primarbereich und die Sekundarstufe I in den Jahren 2003 und 2004 von der Kultusministerkonferenz verabschiedet; die Bildungsstandards für die Allgemeine Hochschulreife folgten im Jahre 2012. Parallel hierzu wurde die Einrichtung des Instituts zur Qualitätsentwicklung im Bildungswesen (IQB) an der Humboldt-Universität zu Berlin angestoßen. Das IQB hat den Auftrag, die länderübergreifenden Bildungsstandards durch geeignete Aufgaben zu operationalisieren und diese zu normieren sowie das Erreichen der Standards regelmäßig 
im Rahmen von Ländervergleichsstudien zu überprüfen. Ferner ist das IQB mit der Entwicklung von Kompetenzstufenmodellen (KSM) und der Weiterentwicklung der länderübergreifenden Bildungsstandards betraut (ebd., S. 12).

Seit 2009 gehört auch die Entwicklung und empirische Erprobung von bildungsstandardbasierten Testaufgaben für flächendeckende Vergleichsarbeiten (VERA, Kompetenztests bzw. Lernstandserhebungen) in der dritten und achten Jahrgangsstufe zu den Aufgaben des IQB. Die Vergleichsarbeiten sollen Impulse für die kompetenzorientierte Unterrichtsentwicklung liefern und die diagnostischen Kompetenzen der Lehrkräfte ausbauen.

Die Erfüllung keiner dieser Aufgaben wäre allein auf Basis der primär diagnostischen und psychometrischen Expertise am IQB möglich. Vielmehr erfordert die Entwicklung von fachspezifischen Test- und Lernaufgaben sowie die Konstruktion von Kompetenzstufenmodellen eine interdisziplinäre Kooperation, in der die Fachdidaktiken einen ganz wesentlichen Beitrag leisten. Daher möchten wir in diesem Aufsatz den Verlauf und das Ergebnis einer solchen interdisziplinären Zusammenarbeit am Beispiel der Entwicklung integrierter Kompetenzstufenmodelle (iKSM) im Fach Deutsch für die Sekundarstufe I darstellen und anhand des Modells für den Kompetenzbereich Lesen konkretisieren.

\section{Die Bildungsstandards der Kultusministerkonferenz}

\subsection{Das Grundkonzept der länderübergreifenden Bildungsstandards}

Bildungsstandards beschreiben fachbezogene Kompetenzen, die Schülerinnen und Schüler bis zu bestimmten Zeitpunkten in ihrer Schullaufbahn im Regelfall erworben haben sollen. Im Kern handelt es sich um Beschreibungen angestrebter Lernergebnisse, also um primär output-orientierte Leistungsstandards. Kompetenzen sind hierbei als Fähigkeiten und Fertigkeiten zu verstehen, die in der Auseinandersetzung mit substanziellen Fachinhalten erworben werden (vgl. Pant et al. 2012, S. 49). Das genaue theoretische Verständnis von Kompetenz kann allerdings in Abhängigkeit von der jeweiligen wissenschaftlichen Disziplin unterschiedlich geprägt sein (vgl. Klieme/Hartig 2007; Grabowski 2014). Den Bildungsstandards der Kultusministerkonferenz liegt ein breit akzeptiertes und häufig verwendetes Kompetenzkonzept zugrunde, das Kompetenzen als Fähigkeiten und Fertigkeiten betrachtet, die sich in konkreten Anforderungssituationen als „Können“ manifestieren. Entsprechend werden die Kompetenzerwartungen der Bildungsstandards im Sinne von Can Do Statements als die Fähigkeit definiert, bestimmte Anforderungen bewältigen zu können, wie zum Beispiel: „Intention(en) eines 
Textes erkennen, insbesondere Zusammenhang zwischen Autorintention(en), Textmerkmalen, Leseerwartungen und Wirkungen“" (KMK 2004, S. 14).

Dieser handlungsorientierte Kompetenzbegriff der Bildungsstandards entspricht der Konzeption von Klieme und Leutner (2006). Sie definieren Kompetenzen als das Ergebnis von Bildungsprozessen und als „kontextspezifische kognitive Leistungsdispositionen, die sich funktional auf Situationen und Anforderungen in einer bestimmten Domäne beziehen“" (Klieme/Leutner 2006, S. 879, Herv. ebd.). Kennzeichnend für dieses Konzept ist eine Abgrenzung von Kompetenz gegenüber Begabung (oder Talent) sowie allgemeiner kognitiver Leistungsfähigkeit. Hiermit geht die Betonung der Erlernbarkeit und Fördermöglichkeit von Kompetenzen und auch eine engere Definition des jeweiligen Expertisebereiches einher, der die Frage ermöglicht, wofür jemand kompetent ist (vgl. Pant et al. 2012, S. 49f.).

Die Bildungsstandards verfolgen verschiedene Ziele. Zunächst dienen sie der Orientierung aller an schulischer Bildung Beteiligten (vor allem der Lehrkräfte, aber auch der Eltern und der Schülerinnen und Schüler selbst) in Hinblick auf verbindliche Zielerwartungen. Denkt man diese Zielstellung weiter, dienen Bildungsstandards auch als Basis für die Überprüfung der Zielerreichung und damit der Leistungsmessung (Assessment), um Problembereiche identifizieren zu können. Primär besteht das Ziel von Bildungsstandards und der auf ihnen basierenden Leistungserhebungen aber darin, die Qualität von Unterricht zu steigern und auf diesem Wege die Kompetenzentwicklung der Schülerinnen und Schüler zu unterstützen. Für das Fach Deutsch impliziert dies einen Unterricht, der Gelegenheiten zur simultanen Steigerung der kommunikativen, literarisch-ästhetischen und methodischen Kompetenzen bereitstellt. Bildungsstandardbasierte Unterrichtsanregungen für die Sekundarstufe I finden sich zum Beispiel bei Behrens und Kollegen (2014).

\subsection{Die Bildungsstandards im Fach Deutsch für die Sekundarstufe I}

Im Fach Deutsch werden in den Bildungsstandards der KMK für die Sekundarstufe I (KMK 2004; KMK 2005) vier zentrale Kompetenzbereiche benannt. Dies sind die Bereiche Sprechen und Zuhören, Schreiben und Lesen - mit Texten und Medien umgehen. Der Kompetenzbereich Sprache und Sprachgebrauch untersuchen nimmt eine Sonderrolle ein. Im Rahmen eines integrativen Ansatzes ist dieser durchgängig mit den anderen Bereichen verbunden, da er Kompetenzen umfasst, die grundlegend für den Erwerb und den Ausbau anderer, sowohl rezeptiver als auch produktiver sprachlicher Kompetenzen sind. Alle vier genannten Bereiche beinhalten als Stützkomponente Methoden und Arbeitstechniken. In den Dokumenten der Kultusministerkonferenz wird der Bereich der Orthografie unter Schreiben subsumiert. Im Rahmen empirischer Studien des IQB wurde jedoch 
sowohl für den Primarbereich als auch für die Sekundarstufe I deutlich, dass es sinnvoll ist, Orthografie in der Diagnostik als eigenen Teilbereich zu behandeln und zusätzlich zu allgemeiner Schreibkompetenz separat zu testen. Ebenso wird Zuhören als separater Teilbereich getestet.

Entsprechend der Schwerpunktsetzung dieses Beitrags betrachten wir nachfolgend vertiefend die Bildungsstandards im Kompetenzbereich Lesen - mit Texten und Medien umgehen. Für diesen Kompetenzbereich enthalten sowohl die Bildungsstandards für den Mittleren Schulabschluss (MSA) wie auch für den Hauptschulabschluss (HSA) eine breite Palette von Teilkompetenzen. Diese beziehen sich allerdings nicht nur auf das Verstehen von Texten, sondern thematisieren auch explizit die Förderung von Leseinteresse und Lesefreude sowie die Ausbildung von Empathie und Fremdverstehen (vgl. KMK 2004, S. 9; KMK 2005, S. 9). Die Schülerinnen und Schüler sollen in die Lage versetzt werden, Informationen in Texten selbständig zu erfassen, zu verknüpfen und mit ihrem Vorwissen in Verbindung zu bringen. Hierfür sollen sie gezielt verschiedene Lesetechniken und Lesestrategien einsetzen. Ferner wird erwartet, dass sich die Schülerinnen und Schüler ein Grundlagenwissen zu Texten, deren Inhalten, Strukturen und historischen Dimension sowie Sprache und Literatur aneignen, über Texte reflektieren, diese bewerten und sich kriterienorientiert mit dem ästhetischen Anspruch von Texten auseinandersetzen. Darüber hinaus sollen die Jugendlichen verschiedene Medien nutzen, um Informationen zu gewinnen und kritisch zu beurteilen. Diese Zielstellungen werden in identischer Weise in den Bildungsstandards für den Hauptschulabschluss (KMK 2005, S. 9) wie für den Mittleren Schulabschluss (KMK 2004, S. 9) formuliert.

Allerdings lassen sich nicht alle formulierten Standards so operationalisieren und in Testaufgaben überführen, dass sie im Rahmen von großangelegten, standardisierten Leistungsmessungen einsetzbar sind. So kann beispielsweise der Standard „über grundlegende Lesefertigkeiten verfügen: flüssig, sinnbezogen, überfliegend, selektiv, navigierend (z. B. Bild-Ton-Text integrierend) lesen" (ebd., S. 13) kaum im Klassenverband im Rahmen von papierbasierten Tests überprüft werden. Unter Berücksichtigung dieser Beschränkungen sind in Abbildung 1 diejenigen Subkomponenten des Bereichs Lesen grau hinterlegt, die in Testaufgaben operationalisiert werden konnten. Deutliche Schwerpunkte liegen dabei auf den Komponenten Literarische Texte verstehen und nutzen sowie Sachund Gebrauchstexte verstehen und nutzen. 
Abb. 1: Subkomponenten des Kompetenzbereichs Lesen in den Bildungsstandards im Fach Deutsch in der Sekundarstufe $I^{1}$

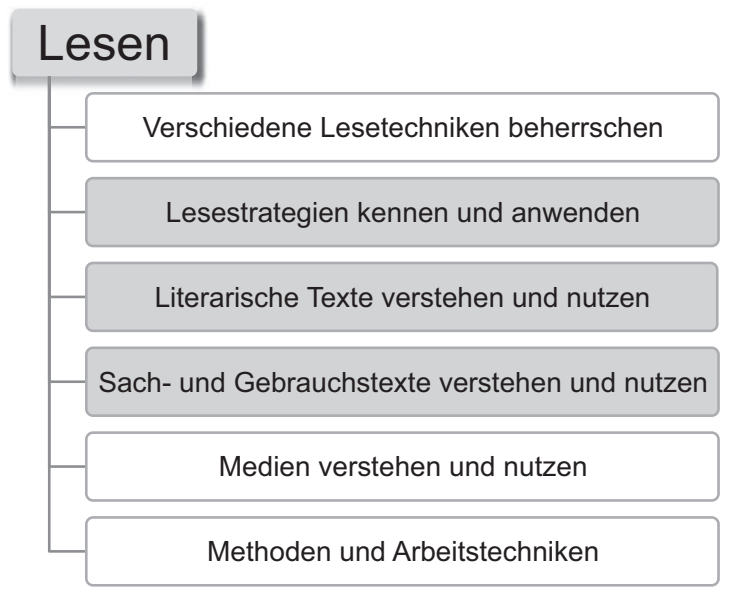

Für die Subkomponenten, zu denen Testaufgaben entwickelt wurden, sind in Tabelle 1 beispielhaft einige Formulierungen der Bildungsstandards für den Hauptschulabschluss und für den Mittleren Schulabschluss aufgeführt und vergleichend nebeneinander gestellt, um Nuancen und Abweichungen in den jeweiligen Zielerwartungen zu illustrieren. Letztere sind in der Tabelle durch Unterstreichungen markiert. Es wird deutlich, dass eine weitgehende Übereinstimmung zwischen den Anforderungen des MSA und des HSA herrschen, die Kompetenzbeschreibungen für den MSA aber an einigen Stellen umfassender formuliert sind.

1 Grau hinterlegt sind diejenigen Komponenten, zu denen im Rahmen der Operationalisierung der Bildungsstandards Aufgaben entwickelt werden konnten. 
Tab. 1: Bildungsstandards des Kompetenzbereichs Lesen - mit Texten und Medien umgehen im Fach Deutsch für den Hauptschulabschluss (HSA) und den Mittleren Schulabschluss (MSA) (IQB 2014, S. 8f.)

\begin{tabular}{|c|c|}
\hline HSA & MSA \\
\hline Literarische Texte verstehen und nutzen & Literarische Texte verstehen und nutzen \\
\hline zentrale Aussagen erschließen, & zentrale Inhalte erschließen, \\
\hline $\begin{array}{l}\text { grundlegende Gestaltungsmittel erkennen } \\
\text { und ihre Wirkungen einschätzen: } \mathrm{z.B} \text {. } \\
\text { Wortwahl, Wiederholung, sprachliche } \\
\text { Bilder, }\end{array}$ & $\begin{array}{l}\text { sprachliche Gestaltungsmittel in ihren } \\
\text { Wirkungszusammenhängen und in ihrer } \\
\text { historischen Bedingtheit erkennen: z. B. } \\
\text { Wort-, Satz- und Gedankenfiguren, } \\
\text { Bildsprache (Metaphern), }\end{array}$ \\
\hline $\begin{array}{l}\text { Handlung und Verhaltensweisen } \\
\text { beschreiben und werten. }\end{array}$ & $\begin{array}{l}\text { Handlungen, Verhaltensweisen und } \\
\text { Verhaltensmotive bewerten. }\end{array}$ \\
\hline $\begin{array}{l}\text { Sach- und Gebrauchstexte verstehen und } \\
\text { nutzen }\end{array}$ & $\begin{array}{l}\text { Sach- und Gebrauchstexte verstehen und } \\
\text { nutzen }\end{array}$ \\
\hline $\begin{array}{l}\text { Informationen zielgerichtet entnehmen, } \\
\text { ordnen, prüfen und ergänzen, }\end{array}$ & $\begin{array}{l}\text { Informationen zielgerichtet entnehmen, } \\
\text { ordnen, vergleichen, prüfen und ergänzen, }\end{array}$ \\
\hline $\begin{array}{l}\text { nichtlineare Texte (auch im } \\
\text { Zusammenhang mit linearen Texten) } \\
\text { auswerten: } z . \text { B. Schaubilder, }\end{array}$ & $\begin{array}{l}\text { nichtlineare Texte auswerten: } \mathrm{z} . \mathrm{B} \text {. } \\
\text { Schaubilder, }\end{array}$ \\
\hline Intention(en) eines Textes erkennen, & $\begin{array}{l}\text { Intention(en) eines Textes erkennen, } \\
\text { insbesondere Zusammenhang zwischen } \\
\text { Autorintention(en), Textmerkmalen, } \\
\text { Leseerwartungen und Wirkungen, }\end{array}$ \\
\hline $\begin{array}{l}\text { Information und Wertung in Texten } \\
\text { unterscheiden: } \underline{\text { z. B. in Zeitungen. }}\end{array}$ & $\begin{array}{l}\text { Information und Wertung in Texten } \\
\text { unterscheiden. }\end{array}$ \\
\hline
\end{tabular}

Bereits die Frage, welche Aspekte der Bildungsstandards operationalisierbar sind und somit in die Testung einfließen können, war Gegenstand von Diskussions- und Aushandlungsprozessen, die als Ausdruck eines Maximenkonflikts verstanden werden können. Zentral war und ist hierbei die Frage, ob in den bildungsstandardbasierten Testungen wie VERA-8 oder den Ländervergleichsstudien primär das erhoben wird, was in geeigneter Form operationalisiert werden kann, oder ob nicht auch Wege gefunden werden können, zusätzlich das zu erheben, was darüber hinaus fachdidaktisch besonders relevant ist. Diese Problematik lässt sich bspw. darauf beziehen, ob bei entsprechenden Testungen überwiegend nach dem Lokalisieren von Einzelinformationen gefragt werden sollte oder ob der Fokus nicht auf der Einbettung und Verknüpfung der Textinformationen in das und mit dem eigenen Vorwissen liegen sollte. Da das Vorwissen der Schülerinnen und Schüler aber nicht nur durch schulische Lerngelegenheiten, sondern 
auch durch familiäre Hintergründe geprägt ist und die eröffneten schulischen Lerngelegenheiten zwischen den Ländern, den Schulen und einzelnen Lehrkräften stark variieren, ist eine Fokussierung auf das Vorwissen aus Gründen der Testfairness und der Vergleichbarkeit nur bedingt möglich. Bereits bei der Frage, was überhaupt messbar ist bzw. sein sollte und welche Konsequenzen bei diesen Einschränkungen mitgedacht werden müssen, war also ein Verständigungsprozess zwischen den beteiligten wissenschaftlichen Disziplinen erforderlich.

\subsection{Konkretisierung und Operationalisierung von Bildungsstandards}

Bei Kompetenzen handelt es sich um theoretische Konstrukte, die nur mit Hilfe von geeigneten Messinstrumenten der Beobachtung zugänglich gemacht werden können (Köller 2008). Um das Erreichen der länderübergreifenden Bildungsstandards im Fach Deutsch in der Sekundarstufe I überprüfen zu können, war es somit zunächst erforderlich, die Bildungsstandards zu konkretisieren und in Testaufgaben zu operationalisieren (vgl. Böhme et al. 2010).

Der Prozess der bildungsstandardbasierten Testentwicklung erfolgt in mehreren Schritten, die nachfolgend mit Fokus auf den Bereich Lesen kurz skizziert werden sollen. Ziel dieses Prozesses ist die Entwicklung reliabler und valider Messinstrumente für die Überprüfung des Erreichens der Bildungsstandards in den Ländervergleichsstudien. Für das Fach Deutsch in der Sekundarstufe I wurde dieser Prozess in den Jahren 2007 bis 2010 durchlaufen. Seitdem findet eine kontinuierliche Aufgabenentwicklung für die Vergleichsarbeiten in der 8. Jahrgangsstufe statt, die einem ähnlichen Konzept folgt.

Für die initiale Testentwicklung wurde zunächst in Vorarbeiten des IQB in enger Kooperation mit Expertinnen und Experten aus der Deutschdidaktik präzisiert, welche der in den Bildungsstandards beschriebenen Kompetenzaspekte im Rahmen eines Large-Scale-Assessments operationalisiert werden können und wie diese zu spezifizieren sind (vgl. Abschnitt 2.2). Auf der Basis internationaler Standards erarbeiteten Mitarbeiterinnen und Mitarbeiter des IQB Hinweise zur Entwicklung und Gestaltung von Testaufgaben (Items). Mit Hilfe dieser Handreichungen wurde ein Aufgabenentwicklerteam im Rahmen von intensiven Schulungen darüber informiert, wie gute Testaufgaben konstruiert sind und wie typische Fehler bei der Itementwicklung vermieden werden können. Diese Phase der Instruktion im Vorfeld der eigentlichen Aufgabenentwicklung war somit stark bildungswissenschaftlich geprägt. Das Aufgabenentwicklerteam setzte sich aus 14 erfahrenen Lehrkräften der Sekundarstufe I aus ganz Deutschland zusammen und arbeitete unter fachdidaktischer Anleitung von März bis Oktober 
2007 an der Auswahl geeigneter Textstimuli sowie der Generierung und Optimierung entsprechender Testaufgaben. Die Aufgaben wurden bereits in dieser frühen Entwicklungsphase durch die Lehrkräfte in ausgewählten Klassen im Rahmen sogenannter Präpilotierungen erprobt, um authentische Schülerlösungen als Grundlage für die Entwicklung von Auswertungsanleitungen und Hinweise für die Optimierung der Items zu erhalten. In einem zweiten Schritt beurteilten und kommentierten Expertinnen und Experten aus der Deutschdidaktik sowie der empirischen Bildungsforschung die vorerprobten Items in Hinblick auf ihre fachdidaktische und testdiagnostische Qualität. Anhand dieser Rückmeldungen wurden die Aufgaben weiter überarbeitet und optimiert.

Auch in dieser Phase der Aufgabenentwicklung waren Aushandlungsprozesse von entscheidender Bedeutung. Diese waren sowohl zwischen der eher praxisorientierten Sichtweite der Lehrkräfte und der eher wissenschaftlich orientierten Perspektive der beratenden Expertinnen und Experten wie auch zwischen den verschiedenen beteiligten Wissenschaftsdisziplinen erforderlich. Art und Inhalt der Aushandlungsprozesse in der Phase der Aufgabenentwicklung möchten wir am Beispiel der eingesetzten Itemformate verdeutlichen.

Zur Erhebung der Lesekompetenz werden in den Tests des IQB Aufgaben eingesetzt, die aus einem Textstimulus und einer Reihe von einzelnen Aufgabenstellungen (Items) bestehen. Als Stimuli werden Sachtexte, Zeitungsartikel, literarische Texte und Diagramme bzw. Schaubilder verwendet. Zu diesen Texten werden Items unterschiedlicher Formate entwickelt. Dies sind zum einen geschlossene Itemformate. Hierzu zählen bspw. Multiple-Choice-Items, bei denen in der IQB-Version zu einer Aussage oder einer Frage vier Antwortoptionen vorgegeben werden, von denen immer nur eine richtig ist. Diese soll ausgewählt und angekreuzt werden. Zu den geschlossenen Itemformaten zählen auch Richtig-Falsch-Items. Bei diesem Itemtyp werden mehrere Aussagen vorgegeben, für die jeweils entschieden werden muss, ob sie richtig oder falsch sind. Diese Entscheidungen werden zeilenweise durch Ankreuzen (richtig oder falsch) getroffen. Eine dritte Variante geschlossener Items stellen Zuordnungsitems dar. Hier müssen verschiedene Elemente aus zwei Gruppen - Problem und Lösung bzw. Frage und Antwort - einander zugeordnet werden. Die Items sind in der Regel so aufgebaut, dass eine vollständige Zuordnung der Elemente nicht möglich ist, da die beiden Gruppen ungleiche Anzahlen an Elementen enthalten. So wird verhindert, dass die letzte Zuordnung bereits durch die Lösung aller vorhergehenden Zuordnungen gegeben ist. Aufgrund der Abhängigkeiten, die zwischen den einzelnen Entscheidungen bestehen, wird nur die richtige Zuordnung aller Elemente als korrekte Antwort bewertet. Ein solches Bewertungsverfahren ist 
vielen Lehrkräften nur schwer zu vermitteln, weil auf diese Weise richtige Teilantworten keine Punkte erhalten.

Zum anderen gibt es neben geschlossenen Itemformaten auch halboffene und offene Formate. Halboffene Items sind Kurzantworten, bei denen die Schülerinnen und Schüler einzelne Informationen, wie etwa Zahlen, Daten, Eigennamen oder einzelne Wörter, selbstständig niederschreiben. Bei offenen Items müssen die Schülerinnen und Schüler eigenständig eine (meist längere) Antwort frei formulieren. Offene Items sind im Allgemeinen schwieriger als geschlossene Items, da hier keinerlei Hinweise auf die richtige Antwort vorliegen und die Beantwortung des Items meist eine eigenständige Inferenz oder aber die sinngemäße Wiedergabe von im Text enthaltenen Informationen erfordert. Des Weiteren sind offene Items meist schwieriger zu bewerten, weil hier interpretiert werden muss, inwieweit die Antworten mit den Erwartungen übereinstimmen.

Der Einsatz geschlossener Itemformate, insbesondere von MC-Items, hat verschiedene psychometrische Vorteile. Dies sind unter anderem die verhältnismäßig kurze, zur Bearbeitung benötigte Testzeit und die hohe Auswertungs- und Interpretationsobjektivität, da die Items automatisch kodiert werden können. Den beteiligten Lehrkräften und auch den fachdidaktischen Expertinnen und Experten waren geschlossene Itemtypen zu Beginn des Aufgabenentwicklungsprozesses allerdings weitgehend unbekannt, was mit einer gewissen Skepsis gegen diese Form der Leistungsmessung einherging. Während sich MC-Items im Verlauf der Testung und insbesondere während der Auswertung der Schülerantworten durch geringen Aufwand auszeichnen, sind diese Items in der Phase der Entwicklung besonders arbeits- und zeitaufwändig. Der Itemstamm soll nach Möglichkeit mit geringem zusätzlichem Leseaufwand auf konstruktrelevante Leseanforderungen abzielen, die sich nicht ausschließlich auf den reinen Abgleich mit im Text gegebenen Einzelinformationen beschränken. Der Attraktor, also die richtige Antwortoption, muss für kompetente Schülerinnen und Schüler eindeutig identifizierbar sein, ohne allein aufgrund der formalen Gestaltung (bspw. durch die Länge der Option oder ihre grammatikalische Gestaltung) hervorzustechen und die Distraktoren, also die drei falschen Antwortoptionen, sollten einerseits plausibel, andererseits nicht verwirrend sein, sondern im Idealfall typische Fehlvorstellungen abbilden. Daher musste zunächst in Gesprächen und auf Basis empirischer Erprobungen von Itementwürfen gemeinsam erarbeitet werden, wie genau gute geschlossene Items gestaltet werden können. Auch dass psychometrische Vorteile die oft zähe und langwierige Entwicklung geschlossener Formate rechtfertigen, musste zunächst ausgehandelt werden. Eine wichtige Erkenntnis hierbei war unter anderem, dass offene Formate zwar in der Entwicklung deutlich weniger Aufwand 
erfordern, dafür aber die Abklärung, welche freie Antwort als (noch) korrekt gelten und von den Jugendlichen erwartet werden kann, deutlich schwieriger und zeitaufwändiger ist. Diese Abklärung umfasste zu jedem offenen Item unter anderem Entscheidungen, welchen Umfang die Schülerantwort mindestens haben muss, ob ein konkretes Beispiel genügt oder ob eine abstraktere Aussage erwartet wird, welchen Stellenwert orthografische und grammatikalische Korrektheit der Schülerantwort haben und wie die jeweils eigenen Erwartungen der einzelnen Aufgabenentwickler mit den Erwartungen der Kolleginnen und Kollegen in Einklang gebracht und dann für Dritte eindeutig schriftlich fixiert werden können. Damit traten die psychometrisch relevanten Aspekte der Objektivität und Reliabilität der Messung auch für die beteiligten Lehrkräfte und die fachdidaktischen Expertinnen und Experten stärker in den Vordergrund. Gleichzeitig wurde für die Vertreterinnen und Vertreter der empirischen Bildungsforschung deutlich, dass es nur bedingt möglich ist, die kognitiven Anforderungen des Reflektierens und Bewertens mit geschlossenen Items und eindeutigen vorformulierten Antworten zu erheben.

Nach der Erstellung eines umfassenden Itempools wurden im Jahr 2007 drei Pilotierungsstudien durchgeführt, die der Überprüfung der Eignung der Aufgaben für Kompetenzmessungen dienten. Anhand der Analyse der Items auf Basis der Item Response Theory (IRT) wurden psychometrisch problematische Items identifiziert und erneut revidiert oder aus dem Itempool entfernt. Weiterhin wurden für offene Items reale Schülerlösungen aus der Pilotierungsstudie für die Optimierung von Auswertungsanleitungen genutzt. Dieser dritte Schritt der Testentwicklung resultierte in einem erprobten Aufgabenpool, der sowohl die Grundlage der nachfolgenden Normierung als auch der späteren Ländervergleiche bildete. Im Frühjahr 2008 wurden die Aufgaben auf der Basis einer national repräsentativen Stichprobe von ca. 7.900 Schülerinnen und Schülern der achten, neunten und zehnten Jahrgangsstufe normiert. Ziel dieser Studien war es, auf der Grundlage empirisch gewonnener Daten eine nationale Skala zu definieren, auf der sowohl die Fähigkeiten der Schülerinnen und Schüler als auch die Schwierigkeiten der eingesetzten Items verortet werden können. Diese Kompetenzskala bezeichnen wir nachfolgend als Bildungsstandardmetrik (BiSta-Metrik).

Auf Grundlage der empirischen Befunde aus den Pilotierungs- und Normierungsstudien sowie unter Bezugnahme auf fachdidaktische Kompetenzstrukturmodelle (vgl. Abschnitt 3.1) wurden in einem abschließenden Schritt des Testentwicklungsprozesses Kompetenzstufenmodelle erarbeitet. Diese bezogen sich zunächst nur auf die Bildungsstandards für den Mittleren Schulabschluss im Fach Deutsch (KMK 2004). Den für die Entwicklung dieser Kompetenzstufenmodelle 
erforderlichen Arbeitsprozess sowie die Erweiterung dieser Modelle zu integrierten Kompetenzstufenmodellen stellen wir in den Abschnitten 3.2 und 4 dieses Beitrags dar.

\section{Die Entwicklung von Kompetenzstufenmodellen}

\subsection{Varianten von Kompetenzmodellen}

Bei der Modellierung von Kompetenzen kann zwischen verschiedenen Perspektiven oder Zielvorstellungen unterschieden werden. Häufig steht die Graduierung einzelner Kompetenzen oder bestimmter Kompetenzbereiche in Niveaus oder Stufen im Zentrum. Kompetenzstufen- oder Kompetenzniveaumodelle stellen dar, auf welcher Stufe, also bei welcher Ausprägung einer Kompetenz, welche konkreten kognitiven und ggf. sprachlichen Anforderungen bewältigt werden können (vgl. Klieme/ Leutner 2006, S. 883). Dies kann beispielsweise empirisch ermittelt werden, indem gelöste Testaufgaben bzw. -items nach ihrer Schwierigkeit geordnet und hinsichtlich ihrer kognitiven Anforderungen analysiert und beschrieben werden. Unabhängig hiervon kann aber auch die Struktur eines Kompetenzbereichs thematisiert werden. Dies ist sowohl aus theoretischer Perspektive als auch auf Basis empirischer Daten möglich. Welche und wie viele verschiedene Dimensionen in einem spezifischen Kompetenzbereich unterschieden werden können, bilden Kompetenzstrukturmodelle ab. Zusätzlich ist auch die Modellierung der Kompetenzentwicklung im Zeitverlauf möglich. Kompetenzentwicklungsmodelle beziehen sich auf die Veränderung der Kompetenz über die Zeit hinweg und darauf, wie sich Kompetenzdimensionen verändern und inwieweit mit fortschreitender Entwicklung komplexere Anforderungen bewältigt, also höhere Kompetenzniveaus erreicht werden (Pant et al. 2012, S. 50).

Ob bei der Kompetenzmodellierung Aspekte der Struktur, des erreichten Niveaus oder der Kompetenzentwicklung im Vordergrund stehen, hängt wesentlich von den Zielen ab, die mit der jeweiligen Modellentwicklung verfolgt werden. Im Zuge der Überprüfung des Erreichens der Bildungsstandards in Ländervergleichsstudien sollen zu jeweils bestimmten Zeitpunkten in den Bildungsverläufen der Kinder und Jugendlichen bilanzierende Aussagen über das Spektrum von Kompetenzen getroffen und Verteilungsaussagen zu den im Bildungssystem erreichten Kompetenzen ermöglicht werden. Zu diesem Zweck werden empirisch fundierte Kompetenzstufenmodelle benötigt, die für jede als relevant angesehene (Teil-)Kompetenz Graduierungen der gemessenen Fähigkeiten in Kompetenzstufen erlauben (ebd., S. 50). Mit Hilfe solcher Modelle ist es möglich, sowohl 
die Testergebnisse von Schülerinnen und Schülern als auch die einzelnen Testaufgaben inhaltlich definierten Kompetenzniveaus zuzuordnen.

Die Entwicklung solcher Kompetenzstufenmodelle erfolgt immer in einem Abstimmungsprozess zwischen Wissenschaft, Schulpraxis und Bildungspolitik. Im Zuge dieses Aushandlungsprozesses wird die kontinuierliche Kompetenzskala in Abschnitte, sogenannte Kompetenzstufen, untergliedert und es werden Aussagen darüber getroffen, welche durch das Aufgabenmaterial konkretisierten Anforderungen von den Schülerinnen und Schülern auf einer bestimmten Kompetenzstufe mit hoher Wahrscheinlichkeit bewältigt werden können. Neben konkreten Aufgabenbeispielen umfassen Kompetenzstufenmodelle immer auch fachdidaktisch und empirisch fundierte Beschreibungen, über welche Kompetenzen Schülerinnen und Schüler, die eine bestimmte Stufe erreicht haben, typischerweise verfügen. Kompetenzstufenmodelle bieten somit eine Möglichkeit, empirisch zu bestimmen, inwieweit Schülerinnen und Schüler die in den Bildungsstandards formulierten Kompetenzerwartungen zu einem bestimmten Zeitpunkt bereits erreicht haben. Wie die Entwicklung von Kompetenzstufenmodellen im Einzelnen erfolgt, wird im nachfolgenden Abschnitt beschrieben.

\subsection{Allgemeine Schritte der Entwicklung von Kompetenzstufenmodellen}

Im Rahmen der Item Response Theory besteht die Möglichkeit, die Fähigkeiten von Personen und die Schwierigkeiten von Items auf einer gemeinsamen Skala zu verorten. Diese Eigenschaft macht man sich bei der Definition von Kompetenzstufen zunutze. So kann beispielsweise ein Item, das einen Schwierigkeitswert von 600 aufweist, in Hinblick darauf analysiert werden, welche kognitiven und ggf. sprachlichen Operationen zu seiner Lösung erforderlich sind. Dies wiederum lässt den Rückschluss zu, dass Personen, die einen Skalenwert von 600 Punkten oder mehr erreicht haben, die zur Lösung dieses Items erforderlichen kognitiven Operationen mit hoher Wahrscheinlichkeit beherrschen. Erweitert man diese Idee, so kann man viele Items in ihrer Schwierigkeit betrachten und Punktwerte (im Sinne von Grenzen) auf der kontinuierlichen Kompetenzskala definieren, bei denen sich die Items hinsichtlich ihrer kognitiven Anforderungen qualitativ verändern, also in inhaltlich beschreibbarer Weise komplexer werden (Pant et al. 2012, S. 52).

Die Festlegung von Schwellenwerten (Cut-Scores) erfolgt im Zuge einer Kompetenzstufensetzung, die im englischsprachigen Raum als Standard-Setting (Cizek 2001; Cizek/Bunch 2007) bezeichnet wird. Hierbei wird die Kompetenzskala in sukzessive Teilbereiche - sogenannte Kompetenzstufen oder Kompetenzniveaus eingeteilt (vgl. Pant et al. 2010). 
In Vorbereitung des Standard-Settings werden die Schwierigkeiten der Items und die Fähigkeiten der Personen zunächst auf einer Skala mit einer geeigneten Metrik dargestellt. Für die Entwicklung der integrierten Kompetenzstufenmodelle wurde bspw. eine Metrik gewählt, bei der die Referenzpopulation einen Mittelwert von 500 Punkten und eine Standardabweichung von 100 Punkten aufweist. Die Referenzpopulation bilden in diesem Fall alle Schülerinnen und Schüler der neunten Jahrgangsstufe, die mindestens den Hauptschulabschluss anstreben, da sich die integrierten Kompetenzstufenmodelle auf die Kompetenzstände dieser Schülerschaft beziehen. Aus den richtigen und falschen Schülerantworten werden die empirischen Schwierigkeiten aller Items ermittelt. Diese Items bzw. eine repräsentative Auswahl der Items werden anschließend in der Reihenfolge ihrer empirischen Schwierigkeit in einem Dokument zusammengestellt, das als geordnetes Itemheft oder Ordered Item Booklet bezeichnet wird.

Die vom IQB durchgeführten Standard-Setting Verfahren stützen sich auf die Einschätzungen und Urteile von Vertreterinnen und Vertretern aus Wissenschaft und Praxis und beziehen Expertinnen und Experten aus den Bereichen Fachdidaktik, Testentwicklung, Schulpraxis und Bildungsadministration ein. Das bereits erwähnte Itemheft stellt die Basis für den Austausch dieser Expertinnen und Experten dar. Auf Grundlage des Itemhefts erörtern alle Beteiligten die kognitiven und ggf. sprachlichen Anforderungen der Items und diskutieren die Konsequenzen verschiedener möglicher Grenzwerte. Die vorgeschlagenen Cut-Scores sowie die kognitiven und sprachlichen Operationen beziehen sich also immer auf die Anforderungen und Merkmale konkreter Items, die im Test und somit auch im Itemheft enthalten sind. Daher besteht der nächste Schritt bei der Entwicklung von Kompetenzstufenmodellen in der Ermittlung dieser kognitiven und sprachlichen Anforderungen der einzelnen Items und in der Klärung der Frage, wodurch sich jeweils weniger anspruchsvolle Items von anspruchsvolleren Items unterscheiden. In diesen Prozess wurden verschiedene theoretische Grundlagen und Vorarbeiten aus anderen Staaten und anderen großen Schulleistungsstudien einbezogen.

Interessant ist hierbei, dass die empirische Schwierigkeit eines Items keinesfalls immer dem aufgrund der Itemeigenschaften erwartbaren Schwierigkeitsniveau entspricht. So können mitunter zwei Items desselben Formats, die sehr ähnliche kognitive und sprachliche Anforderungen stellen, aber aus unterschiedlichen Aufgaben stammen, für die Schülerinnen und Schüler unterschiedlich schwer zu lösen sein. Bei Lese- und Zuhöraufgaben kommt also neben den Merkmalen der Items auch der Einfluss des jeweiligen Stimulustextes (bspw. Art, Umfang und Thema) zum Tragen. Dies kann dazu führen, dass die Bearbeitung von Items zu einem Text, von dem sich die Schülerinnen und Schüler in besonderer Weise 
angesprochen fühlen, leichter fällt, als die Bearbeitung von analogen Items zu einem weniger ansprechenden Text.

Im Zuge der Festlegung der Grenzwerte werden die unterschiedlichen Perspektiven und Prioritäten der beteiligten Personen deutlich. So haben beispielsweise Lehrkräfte oft einzelne, konkrete Schülerinnen und Schüler vor Augen und überlegen, welche Schülerin und welcher Schüler welche aufgabenseitigen Anforderungen bereits mit größerer Sicherheit bewältigen kann. Hierbei spielen individuelle Erfahrungen oftmals eine große Rolle. So wird beispielsweise eine Lehrkraft aus einer sehr leistungsstarken Klasse andere Mindest- und Regelerwartungen äußern als eine Lehrkraft, die in einer Klasse unterrichtet, in der viele Schülerinnen und Schüler schwächere Leistungen zeigen und mehr Unterstützung benötigen. Diese verschiedenen Erwartungshaltungen können zu Unterschieden in den vorgeschlagenen Grenzwerten führen. Aus fachdidaktischer und bildungswissenschaftlicher Perspektive kommen insbesondere die aufgabenseitigen Anforderungen zum Tragen. Hierbei steht das Bemühen im Vordergrund, Gemeinsamkeiten von Aufgaben innerhalb eines bestimmten Schwierigkeitsbereichs zu identifizieren und diese fachsprachlich treffend und gleichzeitig so verständlich wie möglich zu beschreiben. Außerdem kommt eine normative Sichtweise ins Spiel, da die wissenschaftlichen Vertreter oftmals von der Frage ausgehen, was die Jugendlichen aus fachlicher Sicht mindestens oder in der Regel können sollten. Auf Seiten der Vertreterinnen und Vertreter der Bildungsadministration wiederum stellt sich auch die Frage, welche Grenzsetzungen zu welchen Verteilungen von Schülerinnen und Schülern auf die verschiedenen Kompetenzstufen führen. In diesem Kontext ist unter anderem die Frage zentral, wie viele Kinder und Jugendliche einen bestimmten Mindeststandard nicht erreichen und in diesem Sinne als besonders förderbedürftig gelten.

Durch den intensiven Austausch und die Berücksichtigung der jeweils resultierenden empirischen Verteilungen erfolgt eine Konsensfindung der Expertinnen und Experten bzgl. der konkreten Grenzwerte auf der kontinuierlichen Kompetenzskala. Während dieses Arbeitsschrittes wechseln Phasen individueller Überlegungen der einzelnen Expertinnen und Experten mit Phasen des Austauschs und der Diskussion ab. Zentral ist ein Vergleich der individuell gesetzten Grenzwerte mit den von anderen Experten gewählten Grenzwerten. Dieser Vergleich erfolgt sowohl im Rahmen kleinerer Gruppendiskussionen als auch in Paneldiskussionen mit allen Beteiligten. In diesen Gesprächen können die Beweggründe und die jeweils zugrundeliegenden Rationalen für die individuell gesetzten Schwellen erläutert werden und es findet ein Austausch über die jeweiligen Konsequenzen statt. Als Ergebnis dieses iterativen Verfahrens aus individuellen Setzungen und 
Diskussionsphasen resultieren letztlich konkrete Grenzwerte, die den Übergang von einer Kompetenzstufe zur nächsten markieren.

\section{Abb. 2: Schematische Darstellung der Festlegung von Cut-Scores im Standard- Setting-Prozess}

\section{Kontinuierliche Kompetenzskala} niedrige Kompetenz hohe Kompetenz

Cut Score 1 Cut Score 5
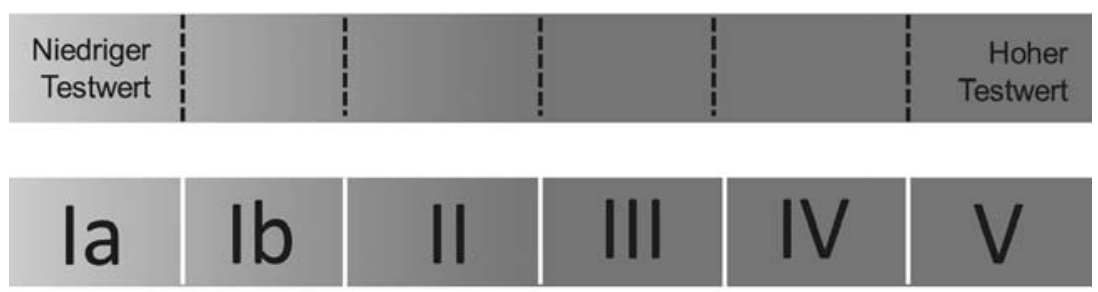

Kompetenzstufen: ordinale Fähigkeitsniveaus

Abbildung 2 zeigt schematisch, dass beim Standard-Setting auf dem Kontinuum der Kompetenzskala durch die Setzung von Cut-Scores festgelegt wird, wo sich jeweils Übergänge zwischen Aufgabengruppen befinden, die abgrenzbare Anforderungen beinhalten und zu deren Lösung zunehmend komplexere kognitive und sprachliche Fähigkeiten notwendig sind. Da die empirischen Schwierigkeiten aller Items bekannt sind und diese ihrer Schwierigkeit nach geordnet im Itemheft aufgeführt sind, kann für jeden Cut-Score zwischen zwei Items ein konkreter Skalenwert bestimmt werden, der als obere bzw. untere Grenze einer Kompetenzstufe dient. Die inhaltliche Beschreibung der Kompetenzstufen erfolgt dann anhand der entsprechenden Fähigkeiten, die erforderlich sind, um die jeweiligen Anforderungen der Items in diesem Skalenabschnitt erfolgreich zu bewältigen. Um zu verdeutlichen, über welche konkreten Kompetenzen Schülerinnen und Schüler auf den jeweiligen Stufen verfügen, werden Könnensbeschreibungen formuliert (z. B. „Schülerinnen und Schülern auf der untersten Kompetenzstufe gelingt es, Einzelinformationen in einem Text zu lokalisieren ...").

In einem letzten Schritt erfolgt die Bestimmung, welcher Skalenabschnitt welchen Kompetenzerwartungen entspricht. Es muss also festgelegt werden, ab welchem Niveau die Vorgaben der Bildungsstandards erfüllt sind. Im Sinne der von Klieme et al. (2007) eingeforderten Differenzierung werden hierbei verschiedene Niveaus festgelegt und Überlegungen zu Mindest- und Optimalstandards 
formuliert. Hierfür wurden die folgenden Festlegungen getroffen (Pant et al. 2012, S. 54f.):

- Mindeststandards beziehen sich auf ein definiertes Minimum an Kompetenzen, das alle Schülerinnen und Schüler bis zu einem bestimmten Bildungsabschnitt erreicht haben sollen. Diese unterschreiten die in den Publikationen der KMK festgelegten Kompetenzerwartungen der Regelstandards. Sie beschreiben jedoch ein Kompetenzniveau am Ende der Sekundarstufe I, von dem angenommen werden kann, dass sich Schülerinnen und Schüler, die dieses erreichen, bei entsprechender Unterstützung erfolgreich in die berufliche Erstausbildung integrieren werden.

- Regelstandards beziehen sich auf Kompetenzen, die im Durchschnitt von den Schülerinnen und Schülern bis zu einem bestimmten Bildungsabschnitt erreicht werden sollen und den von der KMK definierten Kompetenzzielen entsprechen.

- Als Regelstandard plus wird ein Leistungsbereich definiert, der über dem Regelstandard liegt und als Zielperspektive für die Weiterentwicklung von Unterricht angesehen werden kann.

- Optimalstandards beziehen sich auf Leistungserwartungen, die bei sehr guten oder ausgezeichneten individuellen Lernvoraussetzungen und der Bereitstellung besonders günstiger Lerngelegenheiten innerhalb und außerhalb der Schule erreicht werden können und die bei weitem die Erwartungen der Bildungsstandards übertreffen.

\section{Die Entwicklung integrierter Kompetenzstufenmodelle}

\subsection{Bildungspolitische Hintergründe der Entwicklung integrierter Kompetenzstufenmodelle}

Die Bildungsstandards im Fach Deutsch für die Sekundarstufe I sind abschlussbezogen definiert und unterscheiden zwischen Bildungsstandards für den Hauptschulabschluss (KMK 2004) und den Mittleren Schulabschluss (KMK 2005). Dementsprechend wurden in den vergangenen Jahren zunächst auch separate Kompetenzstufenmodelle entwickelt. Für das Fach Deutsch lagen seit 2009 Kompetenzstufenmodelle für den MSA in den Kompetenzbereichen Zuhören und Lesen vor. Seit dem Jahr 2012 war zusätzlich das Kompetenzstufenmodell für den Bereich Orthografie verfügbar. 2014 folgten die Kompetenzstufenmodelle für die Bereiche Schreiben und Sprache und Sprachgebrauch untersuchen. Für den Hauptschulabschluss wurden bislang keine eigenen Kompetenzstufenmodelle entwickelt. 
Die schulstrukturellen Entwicklungen der letzten Jahre machen es allerdings zunehmend schwierig, den von Schülerinnen und Schülern jeweils angestrebten und letztlich erlangten Schulabschluss anhand der jeweils besuchten Schulform vorherzusagen. In vielen Bundesländern existiert die Schulart „Hauptschule“ nicht mehr in der gewohnten Form und Schülerinnen und Schüler, die einen Hauptschulabschluss anstreben, lernen oftmals an Schulen mit mehreren Bildungsgängen oder an integrierten Gesamtschulen, die auch zu anderen Schulabschlüssen hinführen. Oftmals ist es an entsprechenden Schulen bzw. in entsprechenden Bildungsgängen möglich, im Anschluss an den Hauptschulabschluss auch noch den Mittleren Schulabschluss zu erwerben. Daher wird es zunehmend schwieriger zwischen Schülerinnen und Schülern, die den Hauptschulabschluss anstreben, und Schülerinnen und Schülern, die den Mittleren Schulabschluss anstreben, zu unterscheiden. Insgesamt etabliert sich tendenziell eine zweigliedrige Schulstruktur, die neben dem Gymnasium oftmals nur noch eine weitere Schulart umfasst (vgl. Tillmann 2013).

Vor diesem Hintergrund wurde das IQB von Seiten der Bildungspolitik aufgefordert, integrierte Kompetenzstufenmodelle zu entwickeln, die sich auf beide Schülerpopulationen anwenden lassen. Auf Basis dieser Modelle soll es möglich werden, die Kompetenzen aller Schülerinnen und Schüler in den Bildungsgängen, die zum Hauptschulabschluss bzw. zum Mittleren Schulabschluss führen, gemeinsam abzubilden.

\subsection{Die Entwicklung integrierter Kompetenzstufenmodelle im Fach Deutsch}

Das IQB hat von der Amtschefskommission „Qualitätssicherung in Schulen“ im Jahr 2013 den Auftrag erhalten, im Fach Deutsch für der Sekundarstufe I integrierte Kompetenzstufenmodelle (iKSM) zu entwickeln. Diese Modelle sollten sich zunächst auf diejenigen Kompetenzbereiche beschränken, die im Ländervergleich sprachlicher Kompetenzen im Jahr 2015 Gegenstand der Testung sind. Hierbei handelt es sich um die drei Bereiche Lesen, Zuhören und Orthografie. Bis zum Jahr 2014 lagen für das Fach Deutsch nur Kompetenzstufenmodelle für die Population der Schülerinnen und Schüler vor, die einen Mittleren Schulabschluss (MSA) anstreben, nicht jedoch für diejenigen Schülerinnen und Schüler, die einen Hauptschulabschluss (HSA) anstreben. Ziel der Entwicklung integrierter Modelle war es somit, die Kompetenzen aller Schülerinnen und Schüler in den Bildungsgängen, die zum HSA bzw. MSA führen, in einem gemeinsamen Modell abzubilden. Von dieser Definition wurden lediglich diejenigen Schülerinnen und Schüler ausgeschlossen, die sonderpädagogischen Förderbedarf aufweisen und 
zieldifferenten Unterricht erhalten, somit also weder den Mittleren noch den Hauptschulabschluss anstreben. Die Stufenbeschreibungen der iKSM sollten dazu geeignet sein, die erreichten Kompetenzstände in beiden Schülerpopulationen (HSA und MSA) anschaulich und zutreffend darzulegen.

Für das Fach Deutsch erfolgte die Entwicklung der iKSM in einem engen Kooperationsprozess zwischen Mitarbeiterinnen und Mitarbeitern des IQB der Projektgruppe Deutsch in der Sekundarstufe I sowie Mitarbeiterinnen und Mitarbeitern des Mercator-Instituts für Sprachförderung und Deutsch als Zweitsprache der Universität zu Köln. ${ }^{2}$

Die Entwicklungsarbeit an den integrierten Kompetenzstufenmodellen erstreckte sich über das gesamte Jahr 2014. Wichtiges Vorbild für den Entwicklungsprozess und die Gestaltung der iKSM waren die entsprechenden integrierten Kompetenzstufenmodelle für das Fach Mathematik in der Sekundarstufe I. Während eines gemeinsamen Auftakttreffens der Mitarbeiterinnen und Mitarbeitern des IQB sowie des Mercator-Instituts im Februar 2014 wurden die Rahmenbedingungen der Modellentwicklung besprochen, der Projektauftrag konkretisiert und die Arbeitsteilung zwischen den Kooperationspartnern abgestimmt. Nachfolgend wurden vom IQB die für die Modellentwicklung notwendigen, empirischen Grundlagen erstellt. Diese umfassten beispielsweise die angepassten Itembooklets (vgl. Abschnitt 3.2) für die drei Kompetenzbereiche Lesen, Zuhören und Orthografie. Datengrundlage der empirischen Analysen und damit der integrierten Kompetenzstufenmodelle war die Normierungsstudie des IQB aus dem Jahr 2008 im Fach Deutsch (Sekundarstufe I). In dieser Erhebung bearbeitete eine bundesweit repräsentative Stichprobe von Schülerinnen und Schülern der 8., 9. und 10. Jahrgangsstufe an allen Schularten des allgemeinbildenden Schulsystems im Bereich Lesen insgesamt 30 Aufgaben mit 329 Items $^{3}$. Die Testung basierte auf einem Design, in dem jede Schülerin bzw. jeder Schüler nur einen Teil der gesamten Aufgabenmenge bearbeitete (Multi-Matrix-Design). Die dem Kompetenzstufenmodell

2 Da jeder Einzelne zum erfolgreichen Abschluss des Projektes beigetragen hat, sollen alle Beteiligten an dieser Stelle Erwähnung finden. Mitarbeiterinnen und Mitarbeiter des IQB an der Humboldt-Universität zu Berlin: Katrin Böhme (Bildungswissenschaften), Thomas Canz (Linguistik), Susanne Hunger (Rehabilitationswissenschaften), Stefan Schipolowski (Bildungswissenschaften) und Petra Stanat (Bildungswissenschaften). Mitarbeiterinnen und Mitarbeiter des Mercator-Instituts für Sprachförderung und Deutsch als Zweitsprache der Universität zu Köln (alle aus dem Bereich der Sprachdidaktik): Michael Becker-Mrotzek, Necle Bulut, Jörg Jost, Michaela Mörs und Miriam Possmayer.

3 Unter einer Aufgabe wird hier eine Einheit aus Stimulus (z. B. ein Text) und mehreren darauf bezogenen Fragen bzw. Aufgabenstellungen (Items) verstanden. 
zugrunde liegende Metrik hat in der Population der Neuntklässlerinnen und Neuntklässler in Deutschland, die mindestens den Hauptschulabschluss (HSA) anstreben, einen Mittelwert von 500 Punkten und eine Standardabweichung von 100 Punkten.

Im Frühjahr und Sommer 2014 wurden in enger Abstimmung verschiedene Entwurfsfassungen der iKSM entwickelt. Diese wurden mit den empirischen Verteilungen der Schülerinnen und Schüler auf die jeweils vorgeschlagenen Kompetenzstufen verglichen und auf mehreren Arbeitstreffen der Projektbeteiligten detailliert besprochen und überarbeitet. Konkret erfolgte die Arbeit an den iKSM in einem engen Abstimmungsprozess mit wechselnden inhaltlichen Schwerpunkten. So wechselten Phasen mit fachdidaktischem Arbeitsschwerpunkt, in denen die Bestimmung und Beschreibung möglicher Grenzwerte im Vordergrund stand mit primär datenanalytischen Phasen ab, in denen auf Basis empirischer Daten die für die vorgeschlagenen Grenzwerte resultierenden Verteilungen von Schülerinnen und Schülern der Zielpopulation ermittelt wurden. In erstgenannten Phasen waren primär die Mitarbeiterinnen und Mitarbeiter des Mercator-Instituts für Sprachförderung und Deutsch als Zweitsprache der Universität zu Köln aktiv, in Phasen mit datenanalytischem Schwerpunkt lag der Arbeitsmittelpunkt bei den Mitarbeiterinnen und Mitarbeitern des IQB. Die Arbeitsergebnisse wurden regelmäßig ausgetauscht, von dem jeweils anderen Kooperationspartner kommentiert und bei Telefonkonferenzen besprochen. An der Beschreibung der Stufen und der Einbettung des iKSM in einen erläuternden Rahmentext wirkten Mitarbeiterinnen und Mitarbeiter beider Institutionen mit. Der entsprechende Text wurde auf einem ganztägigen Arbeitstreffen mit Vertretern beider Institutionen im September 2014 in Köln finalisiert. Während der intensiven Abstimmungsprozesse zeigte sich wiederholt, dass zwar die Fachsprache der Beteiligten mitunter Verständigungsschwierigkeiten zur Folge hatte, inhaltlich jedoch kaum Differenzen bestanden und in allen Arbeitsphasen stets sehr schnell befriedigende Lösungen gefunden werden konnten.

Im September 2014 wurden die integrierten Kompetenzstufenmodelle in den Bereichen Lesen, Zuhören und Orthografie dann auf der 73. Sitzung der Amtschefskommission vorgestellt. Die Fachreferenten in den Ländern erhielten im Nachgang der Sitzung bis Anfang Dezember die Gelegenheit, die Modelle zu kommentieren und Änderungsvorschläge zu unterbreiten. Die am IQB eingehenden Rückmeldungen wurden in Abstimmung mit den deutschdidaktischen Kooperationspartnern eingehend geprüft und - soweit möglich - in die Modelle eingearbeitet. Diese Überarbeitung wurde detailliert dokumentiert und den Ländervertretern rückgekoppelt. Als Ergebnis dieses kooperativen Entwicklungsprozesses stehen seit Ende 2014 für drei Kompetenzbereiche des Faches Deutsch 
integrierte Kompetenzstufenmodelle für die Sekundarstufe I zur Verfügung und sind auf den Webseiten des IQB unter https://www.iqb.hu-berlin.de/bista/ksm abrufbar (IQB 2014). Die integrierten Kompetenzstufenmodelle werden auch die Grundlage der Berichtlegung für den IQB-Ländervergleich im Jahr 2015 bilden.

Die finalen iKSM umfassen sechs Stufen, wobei das unterste Niveau in Relation zum Kompetenzstufenmodell für den Mittleren Schulabschluss in die Stufen Ia und Ib unterteilt wurde (vgl. Tab. 2). Die unterste Stufe (Stufe Ia) ist nach unten und die oberste Stufe (Stufe V) ist nach oben offen. Die in Tabelle 2 dargestellten Stufengrenzen des Modells für den Kompetenzbereich Lesen wurden in Übereinstimmung mit den Bildungsstandards der KMK abschlussbezogen definiert. Dabei sind die Mindeststandards, Regelstandards, Regelstandards plus und Optimalstandards für den HSA und MSA jeweils um eine Kompetenzstufe gegeneinander versetzt. So bildet z. B. die Kompetenzstufe II den Regelstandard für den HSA und gleichzeitig den Mindeststandard für den MSA ab.

Tab. 2: Stufengrenzen des integrierten Kompetenzstufenmodells im Kompetenzbereich Lesen ${ }^{4}$

\begin{tabular}{|l|l|l|l|}
\hline Stufe & $\begin{array}{l}\text { Punktebereich auf der } \\
\text { Bildungsstandards- } \\
\text { Metrik }\end{array}$ & $\begin{array}{l}\text { Interpretation für den } \\
\text { HSA }\end{array}$ & $\begin{array}{l}\text { Interpretation für den } \\
\text { MSA }\end{array}$ \\
\hline Stufe V & ab 660 Punkten & \multirow{2}{*}{ Optimalstandard HSA } & Optimalstandard MSA \\
\cline { 1 - 2 } Stufe IV & 580 bis 659 Punkte & Regelstandard plus MSA \\
\hline Stufe III & 500 bis 579 Punkte & Regelstandard plus HSA & Regelstandard MSA \\
\hline Stufe II & 420 bis 499 Punkte & Regelstandard HSA & Mindeststandard MSA \\
\hline Stufe Ib & 350 bis 419 Punkte & Mindeststandard HSA & \\
\cline { 1 - 1 } Stufe Ia & bis 349 Punkte & & \\
\hline
\end{tabular}

\subsection{Das Kompetenzstufenmodell zu den Bildungsstandards im Bereich Lesen im Fach}

Um beispielhaft illustrieren zu können, was die Entwicklung integrierter Kompetenzstufenmodelle inhaltlich konkret bedeutet, möchten wir nachfolgend die drei unteren Stufen (Ia bis II) des iKSM für den Hauptschulabschluss und den Mittleren Schulabschluss für den Kompetenzbereich Lesen und die Verteilung der Schülerinnen und Schüler auf die Stufen des iKSM vorstellen. Für die drei ausgewählten Kompetenzstufen wird beschrieben, welche Anforderungen jeweils als zentral und prototypisch erachtet werden können (vgl. IQB 2014).

4 Der Bereich des Regelstandards ist jeweils grau hinterlegt. 


\section{Kompetenzstufe Ia: Lokalisieren und Wiedergeben prominenter}

Einzelinformationen ${ }^{5}$

Schülerinnen und Schülern auf der untersten Kompetenzstufe gelingt es, Einzelinformationen im Text zu lokalisieren und zu verarbeiten. Dies wird vor allem dann bewältigt, wenn diese Informationen in strukturell einfachen und kurzen Texten auffällig platziert sind, etwa wenn sie grafisch hervorgehoben wurden oder zu Beginn oder am Ende von Absätzen stehen. Darüber hinaus können vereinzelt zentrale Einzelinformationen auf der Basis von bereits vorhandenem Welt- und Sprachwissen miteinander verknüpft werden. Bei strukturell einfachen und kurzen Texten gelingt auf dieser Kompetenzstufe auch die Identifizierung des Textthemas, sofern die Bearbeitung dieser Aufgabe durch ein geschlossenes Format erleichtert wird. Längere, komplexere Texte können hingegen auf dieser Kompetenzstufe lediglich auf das Vorhandensein einzelner Informationen hin durchsucht werden, der Aufbau lokaler Kohärenz gelingt jedoch noch nicht.

Schülerinnen und Schüler auf dieser Kompetenzstufe bewältigen überwiegend Aufgaben, bei denen sie aus vorgegebenen Antwortmöglichkeiten die richtige Antwort auswählen müssen, wobei die Distraktoren der gesuchten Information überwiegend nicht ähneln. Seltener gelöst werden Aufgaben, die Kurzantworten - z. B. Zahlen, Daten, Eigennamen und einzelne Wörter - erfordern, sowie Aufgaben, bei denen Antworten eigenständig formuliert werden müssen.

Schülerinnen und Schüler auf dieser Kompetenzstufe verfehlen deutlich die Vorgaben, die in den Bildungsstandards der KMK im Bereich Lesen festgelegt sind. Dies gilt sowohl für die Bildungsstandards für den Hauptschulabschluss als auch für die Bildungsstandards für den Mittleren Schulabschluss. Die Kompetenzstufe Ia beschreibt dementsprechend ein Leistungsniveau, auf dem auch das Bildungsminimum im Sinne des Mindeststandards noch nicht erreicht wird.

\section{Kompetenzstufe Ib: Benachbarte Informationen miteinander verknüpfen}

Schülerinnen und Schüler auf Kompetenzstufe Ib sind in der Lage, lokale Kohärenz zwischen benachbarten und für das Textverständnis zentralen Einzelinformationen aufzubauen. Verstreute Einzelinformationen können in wenigen Fällen miteinander verknüpft werden, sodass der Aufbau globaler Kohärenz ansatzweise gelingt.

5 Die Beschreibungen der Kompetenzstufen entstammen dem unter https://www.iqb. hu-berlin.de/bista/ksm verfügbaren Dokument: „Integriertes Kompetenzstufenmodell zu den Bildungsstandards für den Hauptschulabschluss und den Mittleren Schulabschluss im Fach Deutsch für den Kompetenzbereich Lesen - mit Texten und Medien umgehen" (IQB 2014). 
Die Schülerinnen und Schüler können weniger prominent platzierte Informationen in strukturell komplexeren Texten lokalisieren und verarbeiten. Bei zentralen und prominenten Einzelinformationen gelingt ihnen dies auch dann, wenn diese Information in paraphrasierter Form vorgegeben wird. Einfache Inferenzleistungen (Schlussfolgerungen), die zum Beispiel auf das Handlungsmotiv eines Protagonisten in erzählenden Texten oder auf das zentrale Textthema eines Sachtextes abzielen, können dann bewältigt werden, wenn die Bearbeitung der Aufgabe durch ein geschlossenes Format erleichtert wird. Für das Textverständnis zentrale Inhalte können in Kurzantworten oder im offenen Format sinngemäß wiedergegeben werden.

Die Leistungen der Schülerinnen und Schüler, die diese Kompetenzstufe erreichen, entsprechen noch nicht dem mit den Bildungsstandards der KMK definierten Niveau. Dies gilt sowohl für die Bildungsstandards für den Hauptschulabschluss als auch für die Bildungsstandards für den Mittleren Schulabschluss. Da aber einfache Verknüpfungen des Gelesenen und einfache Inferenzleistungen bereits gelingen und die Fähigkeit zur Wiedergabe zentraler Informationen in eigenen Worten bereits gegeben ist, wird der Mindeststandard für den Hauptschulabschluss auf dieser Stufe erreicht.

\section{Kompetenzstufe II: Informationen miteinander verknüpfen und Textstrukturen erfassen}

Schülerinnen und Schülern auf Kompetenzstufe II gelingt es, mehrere aufeinanderfolgende Einzelinformationen aus strukturell komplexeren, längeren Texten miteinander zu verknüpfen. Durch den Aufbau lokaler Kohärenz werden hier darüber hinaus komplexere Inferenzleistungen (Schlussfolgerungen) bewältigt, die zum Beispiel das Schließen auf etwaige Verhaltensmotive der Figuren bei erzählenden Texten oder das Erfassen zentraler Sachtextaussagen ermöglichen. Darüber hinaus können Wortbedeutungen kontextuell erschlossen und Angaben zu Textsorten und Textstrukturen (z. B. Unterscheidung von Überschrift und Fließtext) gemacht werden, sofern dies durch ein geschlossenes Format der Aufgabe erleichtert wird.

Vereinzelt werden Aufgaben zur Erzählerfunktion bzw. Erzählperspektive literarischer Texte gelöst, dies jedoch auch nur bei geschlossenen Aufgabenformaten. Das Lokalisieren von im Text explizit genannten Einzelinformationen gelingt zunehmend auch dann, wenn ein Teil von ihnen wenig prominent platziert und für das Textverständnis nicht zentral ist. Auch Lokalisierungsaufgaben zu zentralen Einzelinformationen diskontinuierlicher Texte werden gelöst. Insgesamt bewältigen Schülerinnen und Schüler auf der Kompetenzstufe II mehr Aufgaben, bei denen sie ihre Antwort selbstständig formulieren müssen. 
Schülerinnen und Schüler auf dieser Kompetenzstufe zeigen Leistungen, die den Bildungsstandards der KMK für den Hauptschulabschluss entsprechen. Damit erreichen sie den Regelstandard für den Hauptschulabschluss. Die in den Bildungsstandards der KMK definierten Kompetenzniveaus für den Mittleren Schulabschluss werden jedoch noch nicht erreicht. Da aber komplexere Verknüpfungen des Gelesenen und Inferenzleistungen bereits gelingen und strukturelle Merkmale erkannt werden, ist auf dieser Kompetenzstufe auch der Mindeststandard für den Mittleren Schulabschluss erreicht.

In den im Anhang zu diesem Beitrag verfügbaren Abbildungen A1 bis A3 werden zwei Beispieltexte sowie zu jeder Kompetenzstufe je ein Beispielitem gezeigt. Die Items repräsentieren verschiedene Itemformate (vgl. Abschnitt 2.2) und operationalisieren verschiedene kognitive, inhaltliche und sprachliche Anforderungen. Diese sind in Abbildung A1 jeweils oberhalb der Items kurz angegeben.

\section{Verteilung auf die Kompetenzstufen}

In Abbildung 3 ist die Verteilung der Stichprobe der Normierungsstudie des Jahres 2008 auf die sechs Kompetenzstufen des iKSM im Bereich Lesen insgesamt sowie für die HSA-Population und für die MSA-Population wiedergegeben. Die Angaben beziehen sich auf die Zielpopulation aller Neuntklässlerinnen und Neuntklässler unabhängig davon, ob sie den HSA oder den MSA anstreben, allerdings umfasste die Stichprobe im Jahr 2008 keine Schülerinnen und Schüler an Förderschulen. Diese fehlen also in der Verteilung, obwohl sie Bestandteil der Zielpopulation sind, sofern sie einen Regelabschluss anstreben und in diesem Sinne zielgleich unterrichtet werden. Insgesamt verfehlen etwa 11 Prozent aller Schülerinnen und Schüler, die den MSA anstreben, und etwa 23 Prozent aller Schülerinnen und Schüler, die den HSA anstreben, den jeweiligen Mindeststandard (HSA: Stufe Ib; MSA: Stufe II). Diese Jugendlichen benötigen mit großer Wahrscheinlichkeit zusätzliche Unterstützung, um ihre Schullaufbahn mit dem jeweils angestrebten Abschluss erfolgreich beenden zu können. Knapp 38 Prozent aller Neuntklässlerinnen und Neuntklässler mit dem Ziel MSA erreichen noch nicht Kompetenzstufe III und verfehlen damit die in den Dokumenten der KMK formulierten Erwartungen (Regelstandard). Dieser Anteil ist mit 55 Prozent bei den Schülerinnen und Schülern mit dem Ziel HSA noch einmal deutlich höher. Den obersten Leistungsbereich (Optimalstandard) erreichen in der Schülerschaft, die den MSA anstrebt, mehr als $6 \%$ (Stufe V), in der Schülerschaft, die den HSA anstrebt, sind dies nicht einmal $2 \%$ (Stufe IV und V). Insgesamt zeigt sich also, dass die Verteilungen der Neuntklässlerinnen und Neuntklässler, die den HSA bzw. den MSA anstreben, deutlich gegeneinander verschoben sind. Die Schülerschaft, die den MSA anstrebt, erreicht im Mittel höhere Kompetenzstände als die Jugendlichen, die den HSA anstreben. 
Abb. 3: Verteilung der Neuntklässlerinnen und Neuntklässler insgesamt sowie differenziert nach angestrebtem Schulabschluss (HSA bzw. MSA) auf die Kompetenzstufen des integrierten Kompetenzstufenmodells im Kompetenzbereich Lesen

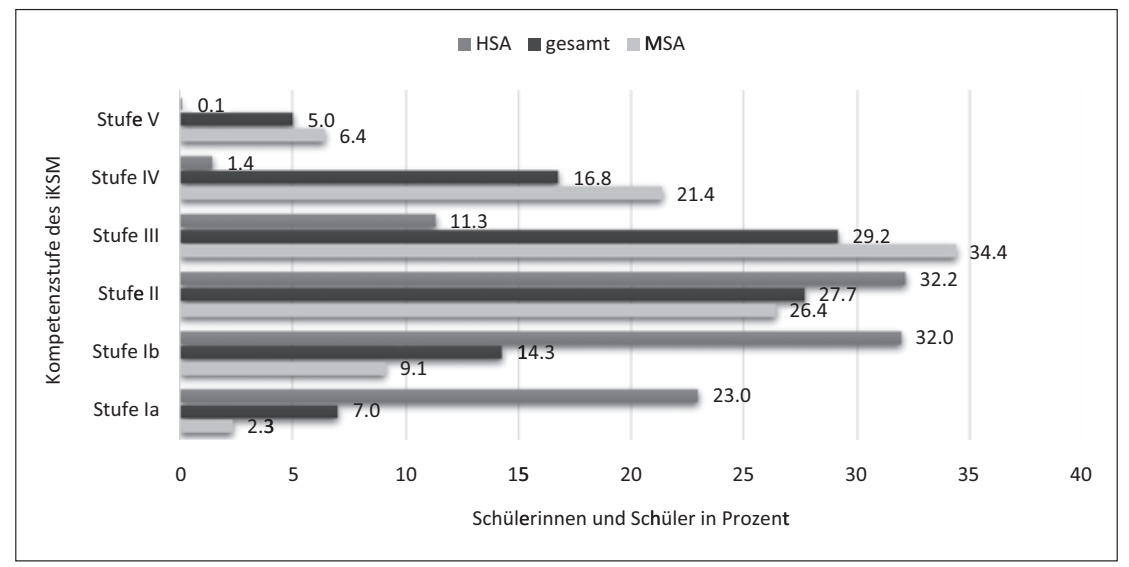

Eine zentrale Botschaft dieser Verteilung ist, dass für leistungsschwächere Schülerinnen und Schüler, die einen Hauptschulabschluss anstreben, im Kompetenzbereich Lesen nach wie vor ein deutlicher Unterstützungsbedarf besteht. Knapp einem Viertel dieser Schülerinnen und Schüler gelingt es in der neunten Jahrgangsstufe bei strukturell komplexeren, längeren Texten nicht, mehrere aufeinanderfolgende Einzelinformationen miteinander zu verknüpfen (Stufe II) oder zumindest benachbarte und für das Textverständnis zentrale Einzelinformationen in Verbindung zu setzen (Stufe Ib). Die Herstellung lokaler Kohärenz als Basis von Inferenzleistungen und die Etablierung globaler Kohärenz als Grundlage eines globalen Textverständnisses sind kaum möglich. Da mit 23\% ein verhältnismäßig großer Anteil der Schülerinnen und Schüler, die einen HSA anstreben, den gesetzten Mindeststandard nicht erreicht, könnte die Frage aufkommen, ob möglicherweise die für die Erreichung der Mindeststandards gewählten Grenzwerte zu hohe Kompetenzerwartungen widerspiegeln. Dies kann sowohl aus bildungswissenschaftlicher als auch als fachdidaktischer Sicht verneint werden. Ein wesentliches Argument ist hier unter anderem, dass die Grenzwerte so gewählt wurden, dass im unteren Leistungsbereich eine trennscharfe Abgrenzung zwischen den Stufenbeschreibungen des integrierten Kompetenzstufenmodells für die Sekundarstufe I und den Stufenbeschreibungen für die Grundschulpopulation erkennbar wird. Schülerinnen und Schüler, die einen Hauptschulanschluss anstreben, sollten also in Hinblick auf ihre Lesekompetenz zwischen den Kompetenzen der Grundschulund der MSA-Population verortet werden. Hierfür ist ein gewisses Anforderungs- 
niveau hinsichtlich der gewählten Cut-Scores erforderlich, das nicht unterschritten werden sollte.

\section{Interdisziplinäre Kooperation als Herausforderung und Chance}

Die interdisziplinäre Zusammenarbeit, hier von Fachdidaktik und Bildungswissenschaft, stellt besondere Herausforderungen, bietet aber auch erhebliche Chancen und Potentiale. Die Herausforderungen bestehen zunächst darin, dass beide Disziplinen im Kern über unterschiedliche Gegenstände, Methoden, Begriffe und Theorien verfügen, die der jeweils anderen Seite nur partiell vertraut und damit in der interdisziplinären Diskussion zugänglich sind. Der Blick auf den gemeinsamen Gegenstand - hier die Kompetenzbereiche des Deutschunterrichts - ist anfangs entsprechend unterschiedlich, ohne dass das allen Beteiligten immer bewusst ist. So ist Lesen in der Fachdidaktik aufs Engste mit Persönlichkeitsbildung, Literatur und kulturellem Gedächtnis verbunden; dabei wurde lange Zeit angenommen, dass mit dem Abschluss des Schriftspracherwerbs auch die Fähigkeit zum sinnverstehenden Lesen erworben ist. Die beim Lesen bedeutsamen kognitiven und sprachlichen Prozesse sowie die hierfür erforderlichen Fertigkeiten standen nicht im Fokus der Fachdidaktik, auch nicht die Möglichkeit, dass größere Teile der Schülerschaft und der Bevölkerung nicht zufriedenstellend lesen können. Das haben erst die Befunde der PISA-Studie des Jahres 2000 (vgl. Baumert et al. 2001; Baumert et al. 2002) und aktuell die Ergebnisse der LEO-Studie (Grotlüschen/ Riekmann 2012) nachdrücklich in das allgemeine Bewusstsein gerückt.

Die groß angelegte Erforschung individueller Fähigkeiten im Rahmen großer Schulleistungsuntersuchungen verlangt nicht nur andere Methoden als bspw. am Individuum orientierte qualitative Zugänge, sondern auch andere Theorien. Das wird besonders deutlich, wenn man prototypisch zwei Messinstrumente gegenüberstellt: Gilt im Deutschunterricht der literarische Interpretationsaufsatz nach wie vor als probates Mittel zur Überprüfung literarischen Verstehens, so nutzen Large-Scale Untersuchungen offene und geschlossene Aufgabenformate (vgl. Abschnitt 2.2). Beide Zugänge haben ihre jeweiligen Vor- und Nachteile, leisten ganz Unterschiedliches und dienen insofern auch unterschiedlichen Zwecken. Diese unterschiedlichen methodischen und inhaltlichen Perspektiven sowie Fachgeschichten bei der gemeinsamen Untersuchung eines Gegenstandes fruchtbar aufeinander zu beziehen, stellt vielleicht die größte Herausforderung der interdisziplinären Kooperation dar. Ist dies gelungen, beginnen konkretere Mühen, etwa bei der Auswahl geeigneter Texte, bei der Formulierung von Aufgaben und Antwortoptionen, bei der Bewertung, was als richtig oder falsch gelten soll und 
wie vor allem empirische Ergebnisse zu werten sind: Was bedeutet es, wenn sehr viele Schülerinnen und Schüler eine Aufgabe nicht beantworten können? Ist sie dann zu schwer - oder verfügen die Schülerinnen und Schüler nicht über die erforderliche Kompetenz?

Die konkrete Zusammenarbeit zwischen den Mitgliedern der Arbeitsgruppe „Integrierte Kompetenzstufenmodelle“ des IQB und des Mercator-Instituts war immer von der Haltung geprägt, die Expertise beider Disziplinen bestmöglich für die Bearbeitung der gemeinsamen Forschungsfragen zu nutzen und keine a priori auszuschließen oder dominant zu setzen. Insofern war die Kooperation nicht nur erfolgreich, sondern auch fruchtbar und lehrreich.

\section{Literatur}

Baumert, Jürgen/Artelt, Cordula/Klieme, Eckhard/Neubrand, Michael/Prenzel, Manfred/Schiefele, Ulrich et al. (Hrsg.) (2002): PISA 2000 - die Länder der Bundesrepublik Deutschland im Vergleich. Opladen: Leske + Budrich.

Baumert, Jürgen/Klieme, Eckhard/Neubrand, Michael/Prenzel, Manfred/Schiefele, Ulrich/Schneider, Wolfgang (Hrsg.) (2001): PISA 2000: Basiskompetenzen von Schülerinnen und Schülern im internationalen Vergleich. Opladen: Leske + Budrich.

Behrens, Ulrike/Bremerich-Vos, Albert/Krelle, Michale/Böhme, Katrin/Hunger, Susanne (Hrsg.) (2014): Bildungsstandards Deutsch: konkret. Sekundarstufe I: Aufgabenbeispiele, Unterrichtsanregungen, Fortbildungsideen. Berlin: Cornelsen Scriptor.

Böhme, Katrin/Neumann, Daniela/Schipolowski, Stefan (2010): Beschreibung der im Ländervergleich im Fach Deutsch untersuchten Kompetenzen. In: Köller, Olaf/Knigge, Michel/Tesch, Bernd (Hrsg.): Sprachliche Kompetenzen im Ländervergleich. Münster: Waxmann, S. 19-25.

Böhme, Katrin/Richter, Dirk/Stanat, Petra/Pant, Hans Anand/Köller, Olaf (2012): Die länderübergreifenden Bildungsstandards in Deutschland. In: Stanat, Petra/ Pant, Hans Anand/Böhme, Katrin/Richter, Dirk (Hrsg.): Kompetenzen von Schülerinnen und Schülern am Ende der vierten Jahrgangsstufe in den Fächern Deutsch und Mathematik. Ergebnisse des IQB-Ländervergleichs 2011. Münster: Waxmann, S. 11-18.

Cizek, Gregory J. (2001): Conjectures on the rise and fall of standard setting: An introduction to context and practice. In Cizek, Gregory J. (Ed.): Setting performance standards: Concepts, methods, and perspectives. Mahwah, NJ: Lawrence Erlbaum Associates Publishers, pp. 3-17. 
Cizek, Gregory J./Bunch, Michael B. (2007): Standard setting: A guide to establishing and evaluating performance standards on tests. Thousand Oaks, CA: Sage Publications Ltd.

Grabowski, Joachim (Hrsg.) (2014): Sinn und Unsinn von Kompetenzen. Fähigkeitskonzepte im Bereich von Sprache, Medien und Kultur. Opladen: Budrich.

Grotlüschen, Anke/Riekmann, Wibke (Hrsg.) (2012): Funktionaler Analphabetismus in Deutschland. Ergebnisse der ersten leo.-Level-One Studie. Münster: Waxmann.

IQB (2014): Integriertes Kompetenzstufenmodell zu den Bildungsstandards für den Hauptschulabschluss und den Mittleren Schulabschluss im Fach Deutsch für den Kompetenzbereich „Lesen - mit Texten und Medien umgehen“. https:// www.iqb.hu-berlin.de/bista/ksm. Abgerufen am 20.04.2016.

Klieme, Eckhard/Leutner, Detlev (2006): Kompetenzmodelle zur Erfassung individueller Lernergebnisse und zur Bilanzierung von Bildungsprozessen. Beschreibung eines neu eingerichteten Schwerpunktprogramms der DFG. In: Zeitschrift für Pädagogik. Jg. 52. H. 6, S. 876-903.

Klieme, Eckhard/Hartig, Johannes (2007): Kompetenzkonzepte in den Sozialwissenschaften und im erziehungswissenschaftlichen Diskurs. In: Prenzel, Manfred/Gogolin, Ingrid/Krüger, Heinz-Hermann (Hrsg.): Kompetenzdiagnostik. Wiesbaden: VS Verlag für Sozialwissenschaften, S. 11-29.

Klieme, Eckhard/Avenarius, Hermann/Blum, Werner/Döbrich, Peter/Gruber, Hans/Prenzel, Manfred (Hrsg.) (2007): Zur Entwicklung nationaler Bildungsstandards. Expertise. Bonn: Bundesministerium für Bildung und Forschung (BMBF).

KMK (2004) = Sekretariat der Ständigen Konferenz der Kultusminister der Länder in der Bundesrepublik Deutschland (2004): Bildungsstandards im Fach Deutsch für den Mittleren Schulabschluss. Beschluss vom 4.12.2003. München: Luchterhand.

KMK (2005) = Sekretariat der Ständigen Konferenz der Kultusminister der Länder in der Bundesrepublik Deutschland (2005): Bildungsstandards im Fach Deutsch für den Hauptschulabschluss. Beschluss vom 15.10.2004. München: Luchterhand.

Köller, Olaf (2008): Bildungsstandards in Deutschland: Implikationen für die Qualitätssicherung und Unterrichtsqualität. In: Meyer, Meinert/Prenzel, Michael/Hellekamps, Stephanie (Hrsg.): Perspektiven der Didaktik. Zeitschrift für Erziehungswissenschaft. Sonderheft 9, S. 47-59.

Pant, Hans Anand/Böhme, Katrin/Köller, Olaf (2012): Kompetenzstufenmodelle für den Primarbereich. Das Kompetenzkonzept der Bildungsstandards und die Entwicklung von Kompetenzstufenmodellen. In: Stanat, Petra/Pant, Hans 
Anand/Böhme, Katrin/Richter, Dirk (Hrsg.): Kompetenzen von Schülerinnen und Schülern am Ende der vierten Jahrgangsstufe in den Fächern Deutsch und Mathematik. Ergebnisse des IQB-Ländervergleichs 2011. Münster: Waxmann, S. 49-55.

Pant, Hans Anand/Tiffin-Richards, Simon P./Köller, Olaf (2010): Standard-Setting für Kompetenztests im Large-Scale-Assessment. Projekt Standardsetting. In: Klieme, Eckhard/Leutner, Detlev/Kenk, Martina (Hrsg.): Kompetenzmodellierung. Zwischenbilanz des DFG-Schwerpunktprogramms und Perspektiven des Forschungsansatzes. Weinheim: Beltz, S. 175-188.

Tillmann, Klaus-Jürgen (2013): Schulstrukturen in 16 deutschen Bundesländern. Zur institutionellen Rahmung des Lebenslaufs (NEPS Working Paper No. 28). Bamberg: Otto-Friedrich-Universität, Nationales Bildungspanel. 


\section{Anhang}

Abb. A1: Beispielitems zu den sechs Stufen des iKSM für den Kompetenzbereich Lesen in der Sekundarstufe I

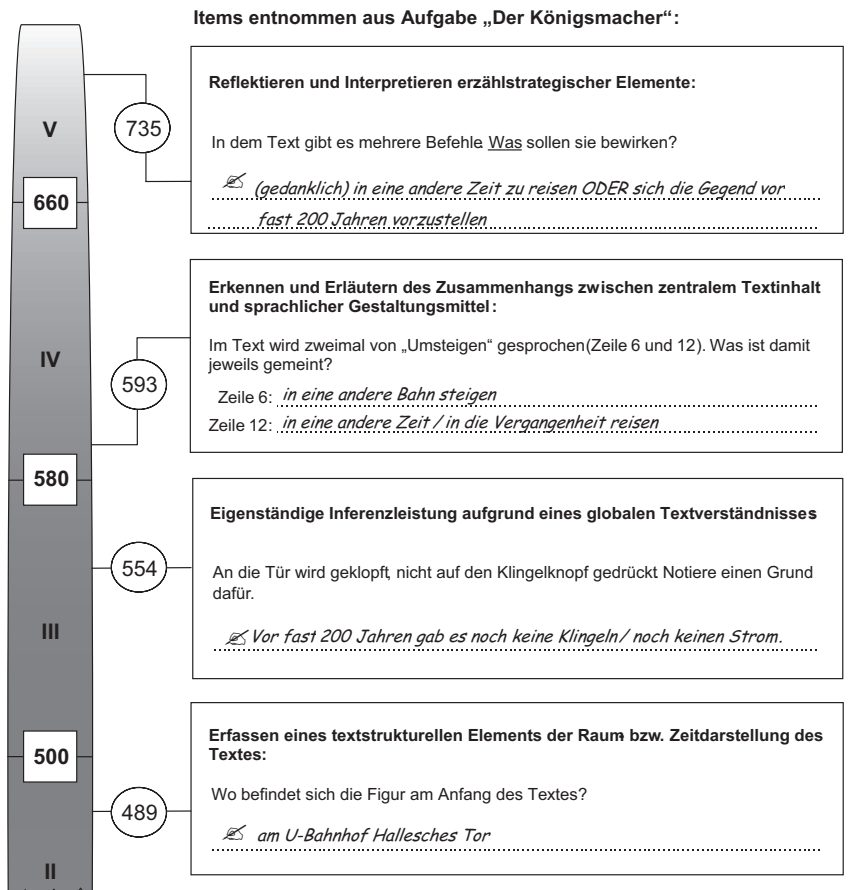

Items entnommen aus Aufgabe „Die lange Berta“:

Benachbarte Informationen miteinander verknüpfen:

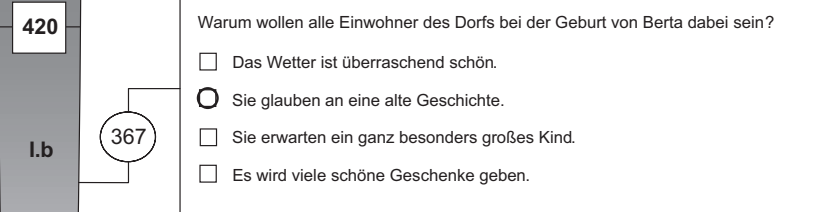

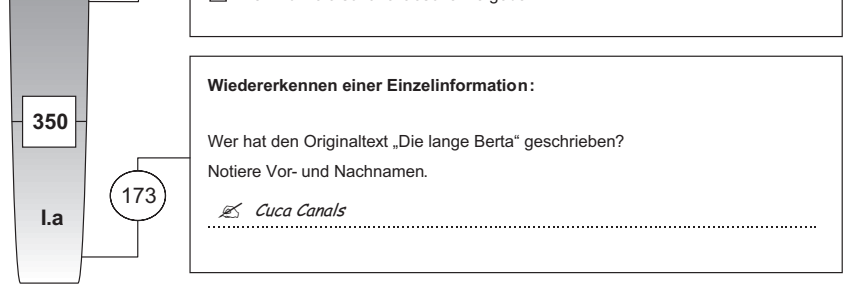

Anmerkung. Aus Platzgründen sind die Aufgabenbeispiele in modifiziertem Layout dargestellt. 
Abb. A2: Textstimulus zu den Beispielitems der Stufen Ia und Ib des iKSM im Kompetenzbereich Lesen

\section{Die lange Berta ${ }^{1}$}

\section{Text nach Cuca Canals}

Berta Quintana war gerade sechzehn Jahre alt geworden und maß einen Meter neunzig. Sie besaß die Schönheit alles Zerbrechlichen, ihre Haut war sehr zart, ihr Gesicht liebreizend, ihre blauen Augen blickten stets ein wenig ängstlich. Aber Berta hasste sich, denn schließlich war es allgemein bekannt, daß es keiner der Männer ihres Heimatortes Navidad dulden konnte, sich von einer Frau über die Schulter blicken zu lassen. Und somit war sie das einzige junge Mädchen im Dorf, das die Liebe noch nicht kannte. Deshalb und weil sie sich außerdem in einem schwierigen Alter befand, wünschte sie sich ein ums andere Mal, nicht auf der Welt zu sein. Aber sie war nun mal geboren worden.

An dem Tag, an dem Berta das Licht der Welt erblickte, regnete es in Strömen. In der Nacht zuvor hatte der Vater, Juan Quintana, den Arzt aus dem Nachbarort Ponsa geholt. Sie kamen beide im Morgengrauen, völlig durchnässt und mit Schlamm bespritzt, in Navidad an.

15 Es wies nichts darauf hin, dass sich die Geburt Bertas von der anderer Kinder unterscheiden sollte, hätte nicht in dem Augenblick, als die Wehen einsetzten, der Himmel aufgeklart und wäre nicht ein wunderschöner Regenbogen erschienen. Die Bewohner Navidads traten aus ihren Häusern und bestaunten ihn mit offenem Mund.

20 Einer alten Legende Navidads zufolge würde nämlich ein Kind, das unter dem Regenbogen zur Welt kam, eine besondere Gabe besitzen. Deshalb geschah es, dass sich im Handumdrehen sämtliche Bewohner Navidads vor dem Haus der Quintanas einfanden, um pünktlich zur Geburt des Kindes anwesend zu sein. Selbst Alberto der Zuckerbäcker, der sehr

25 menschenscheu war, unterbrach seine Arbeit, um jenem Ereignis beizuwohnen, das alle für das wichtigste in der Geschichte Navidads hielten. Die große Erwartung war durchaus berechtigt, schließlich sollte Berta nach dreihundert Jahren das erste Kind Navidads sein, das unter dem Regenbogen geboren wurde.

30 So viele Jahre des Hoffens, Generationen über Generationen, hatten den Bewohnern Navidads Gelegenheit geboten, sich alle nur erdenklichen Wundergaben vorzustellen.

\footnotetext{
Text: Cuca Canals. Die lange Berta. Aus dem Spanischen von Theres Moser. (c) der deutschsprachigen Ausgabe: 1998 Deutscher Taschenbuchverlag, München Textauszug mit Kürzungen S. 13 ff.

${ }^{1}$ Hier wurde die alte Rechtschreibung zum Teil beibehalten (z. B. „daß“).
} 
Abb. A3: Textstimulus zu den Beispielitems der Stufen II bis V des iKSM im Kompetenzbereich Lesen

\section{Der Königsmacher}

\section{Friedrich Christian Delius}

1 Wind weht vorüber, ein kühler Oktoberabend, und wer aus der Untergrundbahn steigt, landet auf einer langgestreckten Brücke. Hallesches Tor ${ }^{1}, 20 \mathrm{Uhr} 41$, die gelben Wagen fahren weg ins Dunkle Richtung Warschauer Straße, der Boden vibriert. Ich will nicht nach Osten, nicht nach Westen, ich folge den Leuten nicht die 5 Treppen hinab.

Umsteigen bitte, ehe die Musik des Eisens wieder anhebt. Ich bleibe am Halleschen Tor am Ende des Bahnsteigs und gebe Befehle.

Denk dir die U-Bahn weg, die Eisengerüste, die Hochhäuser mit Küchen- und Wohnzimmerlicht, den Kanal unten, alles weg. Die Stadt schweige. Rieche den

10 gepflügten Acker, die feuchten Wiesen, nicht Benzin, Fettdunst, Hundekot. Lass die Leute verschwinden oder verkleide sie, wenn du kannst.

Umsteigen bitte, fast zweihundert Jahre zurück, ungezählte Stufen in die Vergangenheit hinunter.

Es ist ganz einfach. Eine neue Landkarte aufschlagen.

15 Stille. Wind weht vorüber, ein kühler Oktoberabend zwischen acht und neun. Ich höre: Hufe schlagen auf einen feuchten Sandweg.

Ich sehe: Ein Pferd galoppiert von Süden heran, den Kreuzberg hinunter. Den Reiter umweht ein weiter schwarzer Mantel. Vor dem Wachposten am Halleschen Tor öffnet er ein Gewand, darunter blitzt eine Generalsuniform auf, der Posten salutiert ${ }^{2}$.

20 Der Reiter passiert ${ }^{3}$ das Rondell ${ }^{4}$ und wendet von der Friedrichstraße nach rechts in die Jacobstraße. Kein Mensch zu sehen, die Straßen dunkel, hinter den Fenstern hier und dort Kerzenlicht. Es ist nicht die Gegend, in der hohe Militärs sich aufhalten. Vor einem der einfachen Häuser steigt der Reiter ab, bindet das Pferd an und klopft an die Tür der Nummer 21. Er ist etwa Mitte dreißig, sieht erschöpft aus, geschlagen,

25 gejagt. Eine ältere Frau öffnet, erkennt ihn, er tritt ein.

Friedrich Christian Delius, „Der Königsmacher" Copyright @ 2001 Rowohlt Berlin Verlag GmbH, Berlin

${ }^{1}$ Hallesches Tor: Name einer U-Bahn-Station in Berlin

${ }^{2}$ salutieren: grüßen

${ }^{3}$ passieren: hier: vorbeireiten

${ }^{4}$ Rondell, das: hier: rundes Beet 
Iris Winkler and Frederike Schmidt - 978-3-631-69285-1

Downloaded from PubFactory at 01/11/2019 10:32:53AM

via free access 
Ulrike Behrens, Sebastian Weirich

\title{
It takes a whole village... Interdisziplinäre Perspektiven bei der Entwicklung eines Testverfahrens zum Zuhören
}

\begin{abstract}
The article shows how the perspectives of linguistics, literature, didactics, psychometrics, acting, administration and technology are interlocked in the construction of test instruments. The project stim.mig has developed and evaluated a new kind of test items for the assessment of listening competency in primary school. Principles of the item construction are being explained and illustrated on the basis of sample items. It becomes clear that interdisciplinary cooperation is indispensable for meeting the state of the art in the various aspects of test development.
\end{abstract}

\section{Einleitung}

Am Beispiel einer einzelnen Testaufgabe zum Hörverstehen bei Grundschulkindern soll gezeigt werden, wie die Perspektiven verschiedener Fachrichtungen und Teildisziplinen im Rahmen der Itementwicklung ineinandergreifen. Besonderes Gewicht kommt einerseits fachwissenschaftlichen und fachdidaktischen Fragen $\mathrm{zu}$, die sich verschränken mit einer psychometrischen Perspektive. Im Fall von Hörverstehensaufgaben sind andererseits die Interpretation und stimmliche Gestaltung von Texten zentral. Es wird deutlich, dass interdisziplinäre Zusammenarbeit unabdingbar ist, wenn man den Anspruch hat, dass möglichst alle Aspekte der Aufgabenentwicklung dem jeweiligen state of the art entsprechen.

Die Zusammensetzung der Projektgruppe kann als interdisziplinär (und zudem international) bezeichnet werden:

- Ulrike Behrens ist Diplompädagogin und wissenschaftliche Mitarbeiterin am Institut für Germanistik der Universität Duisburg-Essen,

- Felix Bertschin ist ausgebildeter Schauspieler und Primarschullehrer sowie Dozent für Theaterpädagogik und Sprecherziehung an der FHNW Liestal,

- Ursula Käser-Leisibach arbeitet als Dozentin für Sprache, Sprachentwicklung und Kommunikation im Institut Vorschul- und Unterstufe der FHNW BruggWindisch,

- Michael Krelle hat Germanistik studiert und ist akademischer Rat im Bereich Sprachdidaktik an der Universität Paderborn, 
- Sebastian Weirich ist als Diplom-Psychologe am Institut für Qualitätsentwicklung im Bildungswesen für die psychometrische Seite der Projekte im Bereich Grundschule zuständig,

- Claudia Zingg Stamm ist Dozentin an der Professur für Deutschdidaktik und ihre Disziplinen im Institut Primarstufe der FHNW Liestal.

Es wird sich im Laufe des Artikels zeigen, dass das noch nicht alle Perspektiven sind, die im Prozess der Aufgabenentwicklung einzunehmen waren und diesen teils erheblich beeinflussten. So waren z. B. aufseiten des IQB als Kooperationspartner für die Testdurchführung im Rahmen der VERA-Pilotierung zahlreiche technisch-administrative Aufgaben zu erbringen, die unmittelbar mit inhaltlichen Fragen der Testentwicklung im Zusammenhang standen. Dazu gehört u. a. auch die teilweise äußerst schwierige Klärung urheberrechtlicher Fragen.

\section{Rahmenbedingungen}

Die Einführung der Bildungsstandards im deutschsprachigen Raum brachte für die Deutschdidaktik nicht nur eine Neuausrichtung von Lehr- und Lernzielen im Sinne einer Kompetenzorientierung mit sich; Fachdidaktikerinnen und Fachdidaktiker waren gleichzeitig erstmals mit der Anforderung konfrontiert, den Erwerb von Kompetenzen und das Erreichen von Standards im Rahmen größerer Erhebungen messbar zu machen. Dies stellte für den Bereich mündlicher Leistungen eine besondere Herausforderung dar: Zum einen lassen sich produktive mündliche Leistungen schon aus technischen Gründen nicht im Rahmen etwa der Bildungsstandards-Normierung, der Ländervergleiche oder VERA-Pilotierungen erheben, denn diese wurden durchweg in Form von paper and pencil-Gruppentestungen durchgeführt. Zum anderen hielten sich die Erfahrungen mit der Messung von Zuhörkompetenzen insgesamt in Grenzen. Erste Ansätze einer Modellierung der Zuhörkompetenz und ihrer Testung waren inspiriert

- von Arbeiten aus dem englischsprachigen Raum (hier z. B. Buck 2001),

- von Vorgehensweisen im Bereich der Fremdsprachdidaktik (vgl. etwa Grotjahn 2000; Nold/Rossa 2007) sowie

- von Formaten der Lesetestung, wie sie seit PISA auch in Deutschland geläufiger wurden (vgl. Baumert et al. 2001).

Insbesondere letzterer Umstand führte dazu, dass die resultierenden Kompetenzstufenmodelle für Lesen und Zuhören erhebliche Parallelen aufweisen, was ihnen gelegentlich die Kritik eintrug, hier werde nicht Zuhörfähigkeit, sondern eigentlich v.a. modalitätsunabhängig Textverstehen modelliert: 
Tatsächlich weisen die Daten der Pilotierungsstudie zur Evaluation der Bildungsstandards für das Fach Deutsch in der Grundschule eine Korrelation auf latenter Ebene von .74 zwischen den Kompetenzbereichen Lesen und Zuhören auf (vgl. Behrens, Böhme u. Krelle 2009). Man findet also im Textverstehen offenbar eine grundlegende Teilkompetenz als Komponente sowohl der Lese- als auch der Zuhörkompetenz. Die naheliegende Kritik, dass mit ,Textverstehen' weder wirklich Zuhörspezifisches noch gar Zuhörtypisches erfasst wird, ist demnach teilweise berechtigt. (Behrens 2010, S. 37)

Zwar gab es Versuche, beispielsweise auch die Nutzung paraverbaler Informationen für den Verstehensprozess in Testaufgaben zu überprüfen. Die Ergebnisse waren aber zunächst empirisch nicht robust genug, um die entsprechenden Fähigkeiten auf Schülerseite sinnvoll in die Kompetenzmodelle zu integrieren. Aus Sicht der Mündlichkeitsdidaktik besteht jedoch kein Zweifel, dass gerade diese Fähigkeiten spezifisch für das Zuhören sind.

\section{Projekt stịm $\cdot$ mig}

Die so umrissene Situation ist der Ausgangspunkt für das deutsch-schweizerische Projekt stim.mig, um das es im Folgenden gehen soll: Ziel war die Entwicklung und Evaluation von Zuhöraufgaben, die neben Verständnisfragen auf der verbalen Ebene auch Items enthalten, die sich ausschließlich auf Basis paraverbaler Informationen lösen lassen.

Die im Rahmen des Projekts entwickelten Aufgaben beziehen sich teils auf literarische Texte, teils auf Radiosendungen zu Sachthemen. Zusätzlich wurden Items entwickelt, die sich nicht auf einen längeren Aufgabenstamm beziehen. Hier müssen z. B. bestimmte Klangmerkmale (beim Flüstern, bei Heiserkeit, beim Kauen etc.) oder die pragmatische Funktion einer Betonung erkannt werden, oder die Testteilnehmer beurteilen authentische Vorleseleistungen von Grundschulkindern. Die Aufgaben wurden im Rahmen der Pilotierung der Vergleichsarbeiten in der dritten Jahrgangsstufe (VERA-3) im Frühjahr 2015 an einer Stichprobe von 5796 Schülerinnen und Schülern evaluiert.

Aufgrund der insgesamt großen Aufgabenmenge, die eine Schülerin oder ein Schüler allein nicht bewältigen könnte, bearbeitet jedes Kind dabei immer nur einen Teil aller Aufgaben. Die Aufgaben werden in Blöcken gruppiert, wobei jeder Aufgabenblock eine unterstellte Bearbeitungszeit von 20 Minuten hat. Jedes Testheft, das ein Kind bearbeitet, besteht dabei aus insgesamt vier Aufgabenblöcken, benötigt also eine planmäßige Bearbeitungszeit von $4 \cdot 20=80$ Minuten. Die Systematik, nach der jeweils vier Aufgabenblöcke für ein Testheft zusammengestellt werden, wird durch das Testdesign definiert. 
Im Rahmen der Studie wurden die Aufgaben des stim.mig-Projekts in das VERATestdesign integriert; jedes Testheft konnte also sowohl Zuhöraufgaben aus stim.mig als auch Lese- bzw. Zuhöraufgaben aus VERA enthalten. Auf diese Weise wurden insgesamt 56 unterschiedliche Testhefte zusammengestellt, wobei sich einige Testhefte in der Auswahl der Aufgabenblöcke teilweise (nicht vollständig) überschneiden. Für jede der an der Studie teilnehmenden Klassen wurde zufällig ein Testheft zur Bearbeitung ausgewählt.

Um eine hohe Durchführungsobjektivität zu gewährleisten, wurde die Studie von instruierten Testleitern durchgeführt. Bei den meisten Zuhöraufgaben wird der gesamten Klasse zunächst ein Text oder Textausschnitt von einer CD vorgespielt, der in seiner Länge zwischen vier und zehn Minuten variiert. Anschließend sind die Kinder aufgefordert, in Stillarbeit zu diesem Text einige Fragen im Testheft zu beantworten. Die Fragen sind so konstruiert, dass eine richtige Beantwortung ein Verständnis des zuvor gehörten Textes bzw. ein korrektes Dekodieren paraverbaler Merkmale voraussetzt.

Nach der Testdurchführung werden die Testhefte wieder eingesammelt und die Antworten der Kinder in einen Datensatz übertragen, wobei jede Antwort eines jeden Kindes wahlweise als 0 (falsch) oder 1 (richtig) kodiert wird. Damit liegt für jedes Item eine Reihe von Itemantworten vor, aus der sich etwa die relative Schwierigkeit des Items (bzw. seine Lösungshäufigkeit) bestimmen lässt.

Insgesamt nahmen an der gemeinsamen Studie (VERA und stim.mig) 5796 Kinder in 299 Klassen teil. Von ihnen bearbeiteten 4158 Kinder in 210 Klassen Aufgaben des stim.mig-Projekts.

Für die Auswertung kommen - wie bei vergleichbaren Schulleistungsstudien üblich - Modelle der probabilistischen Testtheorie (oder Item Response Theory) infrage. Diese Modelle unterstellen eine latente, nicht direkt zu beobachtende Personenfähigkeit (hier: Zuhörkompetenz). Je höher diese Fähigkeit für ein Kind ist, desto höher sollte die Wahrscheinlichkeit sein, dass es eine bestimmte Testaufgabe korrekt löst. Umgekehrt kann für jede Testaufgabe ein Schwierigkeitswert (oder Schwierigkeitsparameter) bestimmt werden: Je größer die Schwierigkeit einer Aufgabe, desto geringer ist die Wahrscheinlichkeit, dass ein bestimmtes Kind diese Aufgabe korrekt bearbeitet. In weiterführenden Analysen kann nun versucht werden, die empirisch ermittelten Schwierigkeiten der Testaufgaben auf ihre spezifischen Eigenschaften zurückzuführen.

Eine erste Sichtung der Itemparameter ergab insgesamt zufriedenstellende Werte, allerdings variieren die Schwierigkeiten der Aufgaben zum Teil erheblich. Über Zusammenhänge mit verschiedenen möglichen Merkmalen der Aufgaben 
und Items kann beim jetzigen Stand der Datenauswertung noch nichts gesagt werden. Ein ausführlicher Projektbericht mit detaillierten Analysen ist in Planung.

In der intensiven Projektarbeit zeigte sich: Die Entwicklung von Zuhöraufgaben ist ein äußerst komplexes Unterfangen, das allen Kooperationspartnern wiederholte Perspektivenwechsel und einen vielfach verschränkten interdisziplinären Diskussionsprozess abverlangt. Die Produktion und gemeinsame Diskussion der Aufgaben erforderte insgesamt vier Projekttreffen und etwa zwanzig Onlinekonferenzen. Im Folgenden sollen die verschiedenen Perspektiven dargestellt werden, die in den verschiedenen Stadien eines solchen Konstruktionsprozesses zu berücksichtigen sind. Für die Aufgabenentwicklung ist das von beträchtlicher Relevanz, weil ihre Berücksichtigung nicht nur den Prozess bereichert, sondern in jedem einzelnen Fall auch dazu führen kann, dass eine in anderen Hinsichten gelungene Aufgabe sich als nicht brauchbar erweist. Im Laufe der Entwicklungsphase wurde etwa die Hälfte der Aufgabenvorschläge in verschiedenen Stadien wieder verworfen. Sehr häufig sind zudem abwägende Entscheidungen zwischen unterschiedlichen Interessen oder Sichtweisen zu treffen. Das betrifft nicht nur den hier exemplarisch nachgezeichneten Entwicklungsprozess, sondern die (Test-) Aufgabenkonstruktion generell - ein Umstand, der zu bedenken ist, wenn Testaufgaben einer kritischen Würdigung durch Dritte unterzogen werden.

\section{Die Beispielaufgabe: Aufregung im Schloss}

Im Folgenden soll anhand eines konkreten Aufgabenbeispiels verdeutlicht werden, wie verschiedene fachliche Perspektiven die Entscheidungen im Verlauf der Aufgabenentwicklung nicht nur beeinflussen, sondern überhaupt erst ermöglichen. Als Beispiel zur Illustration wurde hier die Aufgabe Aufregung im Schloss gewählt. Sie bezieht sich auf einen Auszug aus dem Kinderbuch Die wilde Sophie des Schweizer Schriftstellers Lukas Hartmann. Für die Testaufgabe wurde der Beginn des sechsten Kapitels ausgewählt, als Lesung inszeniert und in Hörbuchform produziert. Der Sprecher Felix Bertschin hatte dabei die Aufgabe, den Figuren unterschiedliche Stimmen zu verleihen, die zur jeweiligen Rolle passen. Entsprechend der auch auf der verbalen Textebene deutlich überzeichneten Figurencharakteristika (die verängstigten Diener, der polternd-autoritäre König, die bodenständige Köchin etc.) sind die verschiedenen Stimmen in der Lesung gut zu unterscheiden.

Der ausgewählte Ausschnitt umfasst 700 Wörter und dauert in seiner Audiofassung siebeneinhalb Minuten. Im Buch gehen ihm fünf Kapitel voraus, in denen das Setting und die Figuren der Geschichte eingeführt werden. Im Mittelpunkt steht Prinz Jan, der vor allem von seinem Vater, König Ferdinand, aus Angst und Sorge um sein Wohlergehen in übertrieben-skurriler Weise behütet und umsorgt 
wird. Ein ganzes Heer von Angestellten, darunter ein „Nebenhergeher“, ein „Insektenjäger“, ein „Kleideranwärmer“ und ein „Lebertranverwalter“, kümmern sich um den Prinzen, der unter diesen Bedingungen zu einem schüchternen und ängstlichen Jungen heranwächst. Dann aber lernt er Sophie kennen, die Titelfigur des Buches, die ganz anders lebt als er und in großer Selbstverständlichkeit all die Dinge tut, die Jan verboten sind.

In dieser Situation beginnt das sechste Kapitel und damit der Textausschnitt, der als Aufgabenstamm für die Testaufgabe fungiert: Die beiden Diener Raimund und Stanislaus wachen auf und entdecken, dass der Prinz, auf den sie offenbar hätten aufpassen sollen, aus seinem Bett verschwunden ist. Noch während sie panisch versuchen zu entscheiden, was zu tun ist, taucht der König auf und will wissen, was los ist. Unter Tränen gestehen sie das Verschwinden ihres Schutzbefohlenen und lösen den befürchteten Wutanfall aus. Der König droht ihnen mit Kerkerhaft und lässt durch zehn Gongschläge Großalarm auslösen, weil er ein Verbrechen befürchtet. Der pragmatische Einwand der Köchin, der Prinz könne freiwillig weggelaufen sein, aus Spaß oder weil er Strafe für ein Vergehen befürchte, wird abgeschmettert, denn dazu habe der Prinz keinerlei Grund. Die angeordnete Durchsuchung des Schlosses ergibt schließlich ein aufgebrochenes Fenster und Blutspuren - für den König der Beweis für eine Entführung. Die Diener entdecken jedoch weitere Hinweise auf die Theorie der Köchin: Die meisten Glasscherben liegen draußen, und ein Baldrianfläschchen ist leer. Der König ist außer sich vor Empörung, dass sein Sohn den Dienern Schlafmittel in den Tee geschüttet haben soll, um ihnen zu entwischen. Er befiehlt den verängstigten Dienern aufgebracht, den Prinzen lebendig zurückzubringen, während er selber einem Zusammenbruch nahe ist.

Hier endet der Textausschnitt. Es folgen insgesamt zehn Testfragen. Diese beziehen sich zur Hälfte auf Inhalte des Textes auf der verbalen Ebene und entsprechen in ihrer Konstruktionsweise den Testaufgaben, wie sie bislang in Normierungsstudien und Vergleichsarbeiten eingesetzt wurden (vgl. auch Behrens et al. 2009). Dies entspricht zum einen der mutmaßlichen Zuhörhaltung der Kinder, die ja zunächst einmal die Geschichte und den Verlauf der Ereignisse verfolgen. Zum anderen gewinnt man so Hinweise darauf, ob die gewählten Texte und neu entwickelten Aufgaben hinsichtlich ihrer Schwierigkeit denen entsprechen, auf deren Basis die aktuellen Modelle konstruiert sind. Diese „verbalen“ Items dienen also der Verknüpfung mit bisherigen Modellen und damit auch der Validierung der Aufgabe. Um diese Vergleichbarkeit zu erreichen, sollen die Items nach Möglichkeit auf unterschiedliche Ebenen des Textverstehens entsprechend den fünf Niveaus des Kompetenzstufenmodells zum Hörverstehen abzielen (vgl. IQB 2013, S. 7-10). 
Als einfach (Stufe 1) gelten dabei Aufgaben zum Wiedererkennen prominenter Einzelinformationen. Anders als bei Lesetexten bezieht sich bei Hörverstehensaufgaben prominent allerdings nicht unbedingt auf die Platzierung beispielsweise zu Beginn eines Textes oder in dessen Titel, wo eine gesuchte Information leicht (wieder) aufzufinden ist. Im akustischen Modus könnte beispielsweise eine Information vom Textanfang im Gedächtnis schon von jüngeren Informationen überlagert sein, wenn am Ende des Hörens Fragen dazu zu beantworten sind. Eine größere Rolle spielt möglicherweise die zeitliche Nähe zum Höhepunkt einer Erzählung, mehrfache Erwähnung im Text, eine besondere akustische Hervorhebung o.ä. Diese theoretischen Überlegungen ergeben sich aus psychologischen bzw. sprachdidaktischen Arbeiten zum Zuhörprozess (z. B. Imhof 2003). Eine empirisch gestützte Modellierung steht aber hier bislang noch aus. Auf den höheren Kompetenzstufen sind aus mehreren Informationen Schlüsse zu ziehen; Items auf Stufe 5 sind $u$. a. gekennzeichnet durch eigenständigere Beurteilungsleistungen. Die Items zu Aufregung im Schloss erweisen sich allerdings (zumindest für die Kinder der Pilotierungsstichprobe) mit Lösungshäufigkeiten zwischen 50\% und 90\% als durchgängig eher leicht - die schwierigsten vier Items dieser Aufgabe rangieren auf den Kompetenzstufen 2 und 3.

Das folgende Beispielitem wird von $84 \%$ der Kinder richtig gelöst und ist somit der Kompetenzstufe 1 zuzuordnen:

Wer sind Raimund und Stanislaus?
A) Zwei Diener
B) Zwei Soldaten
C) Zwei Einbrecher
D) Zwei Prinzen

Die so charakterisierten verbalen Items wurden den Kindern wie bislang üblich in schriftlicher Form vorgelegt; sie mussten also nach dem Hören des Textes gelesen und im Testheft beantwortet werden. Das Design der Gesamtstudie sieht jedoch vor, dass die Items den Kindern teilweise zusätzlich akustisch präsentiert werden. Dadurch sollen die Projektergebnisse unter anderem zu einer weiteren Klärung des Verhältnisses von Lese- und Zuhörfähigkeiten beitragen.

In einem zweiten Teil werden den Kindern Fragen gestellt, die sich auf prosodische bzw. paraverbale Merkmale von Textteilen beziehen. Sie bilden den innovativen Kern des Projekts. Solche Items sind zuerst im Projekt ohrwärts (vgl. Zingg Stamm et al. 2014) als Element eines Lehrmittels zur Diagnose und Förderung der Hörkompetenzen in der Grundschule entwickelt und erprobt worden, dort allerdings zunächst im Rahmen einer einzelnen Aufgabe (d. h. zu einem einzigen Hörtext). Effekte des spezifischen Textes konnten so zunächst nicht überprüft 
werden; auch konnten die neuen Aufgabenformate nur in moderatem Umfang $(\mathrm{N}=200)$ systematisch evaluiert werden. Andererseits erlaubte die vergleichsweise geringe Zahl an Testpersonen, im Rahmen von Beobachtungen in den Versuchsklassen das praktische Funktionieren des neuen Formats und die Einschätzung der Kinder einzuholen. Jenseits von psychometrischen Zugangsweisen kommt hier schulpädagogische Expertise zum Tragen, die in der kaum normierbaren, komplexen Situation im Klassenzimmer in der Lage ist, die je individuellen Umgangsweisen der Kinder mit den verschiedenen Herausforderungen des Tests einzuschätzen. Dieser Weg ist, wiewohl wenig standardisiert, vermutlich der einzige, auf dem man mögliche Störungen wie unerwartete Heiterkeit an bestimmten Textstellen, aber auch motivationale Aspekte wie Langeweile, Überforderung oder andere Irritationen erheben kann.

Das Projekt stịm.mig zeichnet sich demgegenüber dadurch aus, dass erstmals verlässliche Itemkennwerte generiert werden: Es werden zum einen in zwölf zusätzlich entwickelten Aufgaben Texte und Genres systematisch variiert und diese zum anderen an einer großen Stichprobe evaluiert.

Typisch für diese paraverbalen Items des jeweils zweiten Testteils ist, dass die Unterschiede zwischen den Antwortoptionen auf der verbalen Ebene nicht zu erkennen sind. Die richtige Lösung kann nur gefunden werden, indem man prosodische Informationen auswertet. Auch hier wurde ein Teil der Items in den zwei Varianten nur gehört (Variante 1) und gelesen und gehört (Variante 2) evaluiert (Näheres zum Design s.u.). Hierzu ein Beispielitem:

Die Königin ist leicht genervt, weil der König so laut ist. Wo hörst du das am besten? Variante 1: Variante 2:
A)
A)
Weshalb weckst du mich mitten in der Nacht?
C) Weshalb weckst du mich mitten in der Nacht?
D)
D) Weshalb weckst du mich mitten in der Nacht?

Diese Frage „Weshalb weckst du mich mitten in der Nacht?“ kann neutralinteressiert, empört oder auch verzweifelt gemeint sein - entscheidend hierfür ist die stimmliche Gestaltung, die von den Testpersonen richtig zugeordnet werden muss. Alle Items des zweiten Aufgabenteils folgen dieser Logik. Das hat Konsequenzen für die Modalität der Darbietung: Diese paraverbalen Items können nur dann gelöst werden, wenn sie den Kindern zumindest auch akustisch dargeboten werden. 


\section{Die Textauswahl}

Eine der ersten Herausforderungen stellt die Auswahl geeigneter Texte dar. Sie sollen den Interessen der Zielgruppe (hier: von Grundschulkindern) entgegenkommen. In sprachlicher wie in inhaltlicher Hinsicht muss ein Text so gewählt werden, dass er die Verstehensfähigkeiten der Kinder weder übersteigt noch sie unterfordert. Dabei macht es einen Unterschied, ob der Text von den Kindern gelesen oder gehört werden soll. So könnten im Beispieltext etwa Wörter wie steifbeinig, totenblass, Thronsaal für junge Leser erhebliche Hürden darstellen, Begriffe wie Leibarzt, einschläfern, Baldrian wären ggf. zu erläutern. Wenn diese Wörter jedoch nicht selbst erlesen werden müssen, dann gelingt Kindern vermutlich das Verstehen, also die Rekonstruktion von Bedeutung, leichter, da sie kognitiv von den Leseanforderungen entlastet sind. Die stimmliche Gestaltung durch den Sprecher kann dies zusätzlich unterstützen, indem wichtige Stellen besonders hervorgehoben werden oder die herausgehobene Bedeutung einer Phrase (wenn etwa der König sagt: Das ist eine himmelschreiende Verleumdung!) stimmlich markiert wird. Karla Müller schreibt,

dass das Verstehen von Figurenperspektiven mitunter auditiv besser gelingt als beim Lesen. Indem Unbestimmtheitsstellen des Textes durch prosodische Elemente gefüllt werden, kann der emotionale Zustand einer Figur in einer Hörfassung leichter fasslich werden. (Müller 2012, S. 76)

Ein weiteres Kriterium für die Texteignung ergab sich aus den Anforderungen der Prosodie-Items: Es war darauf zu achten, dass im Text gesprochen wird, dass Dialoge vorkommen und möglichst auch verschiedene Emotionen geäußert werden. Andererseits durften nicht zu viele Figuren sprechen, damit jeder einzelnen von einem einzigen Sprecher ein charakteristisches Stimmprofil zugeordnet werden konnte. So konnten in einigen Items den Figuren weitere, im Text nicht enthaltene Äußerungen in den Mund gelegt werden - die Kinder hatten dann zu entscheiden, wer jeweils spricht.

Die Eignung eines Textes zu bestimmen, setzt also die Analyse der inhaltlichen und sprachlichen Potenziale und Herausforderungen voraus. Dies ist zunächst eine literaturwissenschaftliche Anforderung; sofern sich die Eignung auf eine bestimmte Altersgruppe bezieht, kommen zudem sprach- und literaturdidaktische Erwägungen in den Blick. Das ist auch der Fall bei der Auswahl von Texten etwa zur Behandlung im Unterricht. Wenn der Text zu Testzwecken eingesetzt werden soll, ergeben sich aus i. w. S. psychometrischer Perspektive weitere Anforderungen: Zunächst ist zu beachten, dass nicht durch die Thematik einzelne Teilgruppen (z. B. Mädchen, Stadtkinder...) durch die Wahl der Texte systematisch bevorzugt 
werden. Aus dem gleichen Grund ist es wünschenswert, dass ein Text möglichst nicht vielen Kindern (z. B. aus einer bestimmten Region oder auch Bildungsschicht) bereits bekannt ist. Das schließt zum einen (Serien von) Geschichten aus, die als Bücher oder auch in anderen medialen Adaptionen sehr populär sind. Zum anderen kommen aber auch keine Texte infrage, die in verbreiteten Lehrwerken für die Grundschule enthalten sind. Erfolg verspricht vor allem die Sichtung von neu erschienenen (Hör-)Büchern, aber auch von Lektürelisten aus dem (deutschsprachigen) Ausland, weil die Überschneidungen sich nach unseren Erfahrungen in Grenzen halten: Texte, die in der Schweiz sehr verbreitet rezipiert werden, können in Deutschland weitgehend unbekannt sein.

Eine sinnvolle Verwendung der gesamten Bearbeitungszeit erfordert Texte, die bei geringem zeitlichen Umfang möglichst viele sinnvolle Testitems ermöglichen. Das bedeutet, dass im Allgemeinen kürzere Texte längeren vorzuziehen sind, sofern sie inhaltlich ausreichend komplex sind, sodass sich Fragen auf verschiedenen Schwierigkeitsstufen konstruieren lassen. Diese Anforderung bezieht sich nicht nur auf das Kriterium der Testökonomie (d. h. mit möglichst wenig zeitlichem - und finanziellem - Aufwand möglichst viel Information über die Kompetenzen der Testteilnehmer gewinnen; vgl. Moosbrugger/Kelava 2007, S. 20f.), sondern berührt auch Fragen der Validität: Da beim Zuhören mit ansteigender Textlänge vermutlich auch der Anteil der Erinnerungsleistung und ggf. Konzentrationsfähigkeit am getesteten Konstrukt ansteigt, ist es theoretisch möglich, dass Testaufgaben auf Basis sehr langer Texte Anderes testen als solche, die sich auf kürzere Texte beziehen. In der Bildungsstandards-Normierung wurden deswegen Texte mit großem Umfang in mehreren Abschnitten präsentiert, auf die sich dann jeweils nur einige Testfragen bezogen. Der Effekt solcher Maßnahmen ist u. W. aber bislang noch nicht quantifiziert worden. Die im hier beschriebenen Projekt eingesetzten Texte sind nicht länger als zehn Minuten.

Hier war zusätzlich zu bedenken, dass die Aufgaben im Rahmen einer Großstudie eingesetzt werden sollten, die vereinheitlichte zeitliche Abläufe nötig machte: Damit im Rahmen der VERA-Pilotierung die Reihenfolge von Testblöcken zum Lesen und zum Zuhören variiert werden kann, muss die Gesamtbearbeitungszeit für jeden Block gleich lang sein (hier i. d. R. 20 Minuten; s. o.). Sprengt eine Aufgabe diesen zeitlichen Rahmen, so muss ggf. der Text sinnvoll gekürzt oder auf Items verzichtet werden. Dass aus urheberrechtlichen Gründen Kürzungen und Veränderungen innerhalb der Texte in der Regel nicht infrage kommen, schränkt die Auswahl an geeigneten Texten zusätzlich ein.

So kann es passieren, dass ein großer Pool an prinzipiell geeigneten Texten erheblich reduziert werden muss, weil einzelne Texte 
- thematisch einzelne Gruppen von Kindern systematisch bevorzugen oder benachteiligen,

- Grundschulkinder beim Zuhören unterfordern würden,

- zu wenig Material für Items auf verschiedenen Schwierigkeitsniveaus bieten,

- zu lang sind und nicht sinnvoll gekürzt werden können.

\section{Der Textausschnitt}

Erfahrungsgemäß können viele (literarische) Texte die oben genannten Bedingungen nicht erfüllen. Je nach Gestaltung enthält eine Lesung pro Minute etwa 100 Wörter; der Beispieltext Aufregung im Schloss dauert in seiner Audiofassung ca. siebeneinhalb Minuten und entspricht damit in etwa dem angestrebten Textumfang. Selbst dieser Umfang wird in den unsystematisch erhobenen Protokollen, in denen die Testleiter besondere Beobachtungen aus den Testsitzungen notieren, gelegentlich als für die Kinder zu lang vermerkt. In sich abgeschlossene Texte dieser Länge finden sich aber zumeist für ein jüngeres Publikum, zum Beispiel in Form von Bilderbüchern oder etwa den Pixi-Büchern des Carlsen-Verlags. Typischerweise machen hier aber die Illustrationen einen beträchtlichen Anteil des Buchinhalts aus, der für den Textinhalt nicht ohne Weiteres verzichtbar ist. Zudem erweisen sich die Geschichten in ihrer Erzählstruktur häufig als zu eindimensional, sodass es schwerfällt, Fragen auf höheren Anforderungsniveaus zu formulieren.

Eine Alternative zu solchen abgeschlossenen Geschichten sind Auszüge aus längeren Texten. Häufig eignet sich nur der Beginn einer Erzählung, weil im späteren Verlauf auf Informationen aus der Exposition zurückgegriffen wird und der Ausschnitt ohne diese Informationen nicht verständlich ist. Im Textauszug, der hier zur Illustration dient, ergibt sich diese Schwierigkeit nur an den zwei Stellen, an denen König und Königin bei ihren Vornamen - Ferdinand und Isabella - genannt werden. Im einen Fall wird gleich im Folgesatz klar, um wen es sich handelt: „Mein Sohn ist entführt worden!“ Dass es sich bei Isabella um die Königin handelt, kann angenommen werden. Es ergibt sich nicht zwingend aus dem Text, ist aber für das Textverständnis auch nicht von großer Bedeutung. Insofern kann der Ausschnitt gehört werden wie der Beginn einer Geschichte, freilich mit einem offenen Ende, das auf eine notwendige Fortsetzung hinweist. Die Vorgeschichte, beginnend noch vor der ersehnten Geburt des Prinzen Jan, die in den vorausgehenden fünf Kapiteln dargestellt wird, ist für das Verständnis dieses Ausschnittes nicht erforderlich. Der Buchtitel Die wilde Sophie musste hingegen gegen eine passendere Überschrift ausgetauscht werden, um die Zuhörenden nicht zu verwirren. 
Neben den Auswahlkriterien der passenden Textlänge und einer gewissen Abgeschlossenheit ist für erzählende Texte entscheidend, dass zentrale Strukturelemente von Geschichten enthalten sind. Hierzu zählt neben der angemessenen, ggf. sukzessiven Einführung von Setting und Personal eine Komplikation bzw. ein Planbruch als Voraussetzung der Erzählwürdigkeit (vgl. Quasthoff 1980, S. 57-67), im Falle des Beispiels das Verschwinden des Prinzen, das die weiteren Aktivitäten auslöst.

\section{Die Itemkonstruktion}

Die Normierungsstudie im Rahmen der Evaluation der Bildungsstandards führte für den Kompetenzbereich Zuhören im Grundschulalter zur Konstruktion eines fünfstufigen Kompetenzstufenmodells (vgl. IQB 2013). Die Beschreibungen der einzelnen Stufen enthalten neben den jeweiligen kognitiven Anforderungen auch Hinweise zum Format der Items, die auf der jeweiligen Stufe typischerweise gelöst werden. So werden auf der Stufe I „die Anforderungen [...] vor allem dann bewältigt, wenn die Aufgaben ein Multiple-Choice-Format haben“ (IQB 2013, S. 7); auf Stufe III werden „lenkende Hinweise, wie sie in Multiple-Choice-Aufgaben enthalten sind, [...] dabei nicht mehr durchgängig benötigt, um die Anforderungen zu bewältigen“ (IQB 2013, S. 9), und auf der Stufe V heißt es: „Die Schülerinnen und Schüler bewältigen die Anforderungen dabei meist auch im Rahmen von komplexen offenen Aufgaben“ (IQB 2013, S. 10). Insgesamt zeigt sich, dass geschlossene Aufgabenformate dazu beitragen, Items leichter zu machen, während ein offenes Aufgabenformat eine Aufgabe tendenziell schwieriger macht. Um den Einfluss des Aufgabenformats zu reduzieren und von anderen Effekten abgrenzen zu können, wurde im Projekt durchgängig auf geschlossene Itemformate gesetzt. Dabei handelte es sich in aller Regel um Multiple-Choice-Aufgaben mit vier Antwortoptionen und genau einer richtigen Lösung. Gelegentlich wurden auch Wahr-Falsch-Aufgaben eingesetzt, bei denen jeweils vier zu bewertende Aussagen zu einem Item gebündelt werden, um die Ratewahrscheinlichkeit zu reduzieren.

Für eine Mischung aus geschlossenen und offenen Aufgaben spricht in der Regel neben einer abwechslungsreicheren Testgestaltung vor allem die Überlegung, dass nur in (halb-)offenen Aufgabenstellungen, also Aufgaben, in denen die Testteilnehmer selbst formulierte Antworten geben müssen, Wissen erhoben wird, das die Schülerinnen und Schüler eigenständig produzieren können (statt auf bloßes Wiedererkennen zu setzen). Oft wird auch argumentiert, dass komplexere kognitive Prozesse, mithin tiefergehende Verstehensleistungen im MultipleChoice-Format nicht ermittelt werden können. 
Andererseits hat der Einsatz von halboffenen und offenen Items den Nachteil, dass die Stärke des Effekts, den das Format auf die Lösungswahrscheinlichkeit hat, kaum bestimmt werden kann. Zudem gibt es administrative und auch finanzielle Konsequenzen: Während die Auswertung geschlossener Items sehr eindeutig mithilfe von Schablonen, unter Umständen sogar maschinell erfolgen kann, müssen von den Testteilnehmern selbst formulierte Lösungen von eigens geschulten Personen beurteilt werden. Sowohl die Erstellung und Erprobung der dafür erforderlichen Auswertungsanleitung als auch die Schulung der Kodiererinnen und Kodierer erfordert erhebliche zeitliche und personelle Ressourcen, die in der Gesamtplanung und -kalkulation entsprechender Projekte zu berücksichtigen sind. Das trifft insbesondere dann zu, wenn in einem größeren Projekt eine Gruppe von Kodierenden dahin geführt werden muss, Schülerlösungen gleichsinnig zu beurteilen. Eine zu geringe Übereinstimmung in den Kodierungen derselben Lösung weist auf unpräzise Auswertungskriterien hin, die selbst im Falle eines einzelnen Raters zu mangelnder Reliabilität der Testergebnisse führen könnte. Dies ist ein Beispiel für den oben angesprochenen Abwägungsprozess, der hier (neben den ökonomischen) zwei Aspekte von Validität betrifft: Offene Items können die Validität erhöhen, weil alltagsrelevantere Wissensarten in den Blick kommen. Andererseits kommen aber auch zusätzliche Kompetenzen (hier: Schreiben) ins Spiel, deren Einfluss auf das Lösungsverhalten nicht quantifizierbar ist.

Die Entscheidung für ein durchgängig geschlossenes Aufgabenformat, wie sie im hier vorgestellten Projekt getroffen wurde, macht es erforderlich, zu jeder Frage neben der richtigen Antwort drei plausible, aber eindeutig falsche Antworten (Distraktoren) zu formulieren. Inwiefern das gelungen ist, wird die Itemanalyse ergeben: Die Häufigkeit, mit der die Kinder ein Item richtig beantworten, gibt Auskunft über dessen Schwierigkeit; inwieweit die Distraktoren gut gewählt wurden, zeigt sich, wenn man das Antwortverhalten bei ungelösten Items betrachtet: Idealerweise sollten alle falschen Optionen etwa gleich häufig angekreuzt werden. Der Grund ist ein statistischer: Falls sich eine der falschen Antworten als abwegig erwiese und von kaum jemandem gewählt würde, blieben faktisch nur drei statt vier Optionen, was die Ratewahrscheinlichkeit von 25\% auf 33\% erhöhen und damit das Item im Vergleich deutlich leichter machen würde, ohne dass die erforderliche Zuhörleistung tatsächlich komplexer wäre.

\section{Die Testfragen}

Eine Geschichte zu verstehen bedeutet, auf Rezipientenseite eine mentale Repräsentation der Textinhalte zu konstruieren, die der Intention aufseiten des Autors entspricht (vgl. Schnotz 1996, S. 972). Für die Testkonstruktion ist zu bestimmen, 
welches die für solches Verstehen relevanten Inhalte sind, was man also von kompetenten Rezipienten an Verstehensleistungen erwarten kann bzw. muss. Hier macht es nach sprachdidaktischen Einsichten in die Natur und Charakteristika mündlicher Kommunikation für die Testkonstruktion einen wesentlichen Unterschied, ob der Text gehört oder gelesen wird: Während Lesetestfragen durchaus auch auf eine eingehendere, zielgerichtete Re-Lektüre abzielen können, ist dies beim Zuhören nicht der Fall. Wegen der Flüchtigkeit akustischer Signale (vgl. Fiehler 2014; Fiehler et al. 2004) müssen Zuhöritems solche Informationen und Schlussfolgerungen adressieren, die Zuhörende typischerweise bereits während des Hörens in ihre mentale Repräsentation integrieren. So verbieten sich - um nur ein Beispiel zu nennen - Fragen nach ausschmückenden, aber inhaltlich nebensächlichen Details. Beim Entwurf von Testfragen ist es daher ratsam, sich Notizen zu wichtigen Textinhalten möglichst bereits nach dem ersten Hören zu machen bzw. Fragen, die auf Basis eines (schriftlichen) Transkripts formuliert wurden, in dieser Hinsicht kritisch prüfen zu lassen.

Im Unterschied zum auch empirisch gut erforschten Leseverstehen stecken Modelle zur Zuhörkompetenz nach wie vor in den Kinderschuhen. Unstrittig ist, dass Rezipienten beim Zuhören komplexe, multimodale Informationen auswerten, um Sinn zu rekonstruieren. Die bislang empirisch überprüften Modelle berücksichtigen dabei zu einem wesentlichen Anteil den Aspekt des Textverstehens. Vergleichende Untersuchungen von Leistungen in Lese- und Zuhörtestungen (vgl. Böhme et al. 2010) weisen auf erhebliche Überschneidungen beider Modalitäten hin, die derzeit am ehesten dadurch erklärt werden können, dass man eine modalitätsunabhängige bzw. -übergreifende Textverstehenskompetenz annimmt. Zusammenfassend schreibt Böhme,

dass sowohl für das Hör- als auch für das Leseverstehen allgemeine Aufgabenmerkmale, die sich auf das Textverstehen beziehen, bei der Erklärung der Itemschwierigkeit einen wichtigen Stellenwert einnehmen. Um größere Teile der Varianz in der Itemschwierigkeit erklären zu können, sind jedoch Merkmale nötig, die spezifische Eigenschaften des Hörbeziehungsweise Leseverstehens beschreiben. Diese Befunde sind erwartungskonform, da in der theoretischen Betrachtung der beiden rezeptiven Sprachkompetenzen betont wird, dass die kognitiven Informationsverarbeitungsprozesse weitreichende Ähnlichkeiten aufweisen, auf der Ebene der Rezeption jedoch die verschiedenen Inputstimuli (visuell vs. auditiv) berücksichtigt werden müssen. (Böhme 2011, S. 92)

$\mathrm{Zu}$ den zuhörspezifischen Verstehensleistungen gehört ohne Zweifel die Bedeutungsrekonstruktion aufgrund paraverbaler Charakteristika gesprochener Sprache (vgl. zusammenfassend etwa Imhof 2003, S. 30-33). Dazu gehören zahlreiche Merkmale wie etwa Sprechtempo bzw. -dehnung, Pausen, Stimmklang und Tonhöhe, Lautstärke und Artikulation sowie Betonung (Akzent) auf Silben-, Wort-, (Teil-) 
Satz- und Textebene, die jeweils für sich genommen, aber auch in Kombination die Bedeutung des Gesprochenen festlegen und z. T. in Nuancen differenzieren (vgl. auch Bose et al. 2013, S. 39). Will man die Fähigkeit überprüfen, eine so verstandene eigentliche Bedeutung zu ermitteln, so setzt das eine gewisse Eindeutigkeit der Zuordnung voraus. Dass der Zusammenhang zwischen paraverbalem Mittel und emotionalem Gehalt einer Äußerung nicht beliebig ist, zeigt sich in den Arbeiten von Baldur Neuber (2010): Seine Probanden hatten das Pseudowort katakamala jeweils freundlich, sachlich, eindringlich oder ärgerlich zu sprechen. Dabei produzierten sie melodische Konturen mit charakteristischen Merkmalen, die als Prototypen des Ausdruckes zumindest einiger grundlegender Emotionen gelten können, und die überindividuell mit großer Trefferwahrscheinlichkeit reidentifiziert wurden.

Wenn Texte eigens für die Testentwicklung als Hörtexte produziert werden, ist dies bereits bei der akustischen Interpretation des Textes zu berücksichtigen, denn ob eine Textstelle als bedeutsam in Erinnerung bleibt, hängt auch davon ab, wie der Sprecher bzw. die Sprecherin sie gestaltet: Derselbe Inhalt auf der verbalen Ebene kann je nach stimmlicher Gestaltung sehr Unterschiedliches bedeuten.

Dieser Aspekt der Zuhörkompetenz ist bislang zwar theoretisch modelliert und auch in einigen Studien zur Grundlagenforschung empirisch fundiert worden (für einen Überblick vgl. Behrens 2010, S. 44f.), konnte bislang aber noch nicht in Testverfahren integriert werden. Das führte in der Vergangenheit zu testbasierten Modellierungen, die aus zuhördidaktischer Sicht als unterkomplex angesehen werden müssen. Aus Gründen der Testkonstruktion konnte die Fähigkeit, aufgrund paraverbaler Merkmale zwischen verschiedenen Bedeutungen zu unterscheiden, bislang nicht in die Beschreibung der vorliegenden Kompetenzstufenmodelle aufgenommen werden.

Diese Lücke soll mithilfe der Projektergebnisse geschlossen werden. Ganz bewusst wurden Testaufgaben hergestellt, die neben den bislang üblichen Formaten auch solche Items enthalten, in denen die Testpersonen paraverbale Merkmale im angedeuteten Sinne verstehen müssen. Dabei wurden verschiedene paraverbale Itemtypen erprobt, die hier nur knapp charakterisiert werden sollen (für detailliertere Information vgl. die technische Dokumentation).

Typ 1: Eine Äußerung wird auf vier unterschiedliche Weisen gesprochen. Es ist die Option auszuwählen, die einer im Itemstamm vorgegebenen Sprecherintention (hier: leicht genervt) entspricht:

Die Königin ist leicht genervt, weil der König so laut ist. Wo hörst du das am besten?

A) Weshalb weckst du mich mitten in der Nacht?

B) Weshalb weckst du mich mitten in der Nacht? 
C) Weshalb weckst du mich mitten in der Nacht?

D) Weshalb weckst du mich mitten in der Nacht?

Es wurde darauf geachtet, dass die Äußerung zwar zum Kontext der Geschichte passt, aber im Text nicht identisch enthalten ist. Auf diese Weise kann ausgeschlossen werden, dass Testpersonen nur ein bestimmtes klangliches Gebilde wiedererkennen, indem sie bemerken, dass sie die richtige Lösung schon einmal gehört haben.

Im Falle dieses Itemtyps ist es unabdingbar, dass der Hörtext selbst produziert wurde, denn der Interpret des Textes muss auch die vier Antwortoptionen mit den verschiedenen emotionalen Färbungen einsprechen. Erfahrungsgemäß ist hier eine enge Zusammenarbeit mit den Sprecherinnen und Sprechern erforderlich, die ggf. mehrfache Rückkopplungen ermöglicht. (Bewährt hat sich, in einer gemeinsamen Sitzung aus mehreren aufgezeichneten Versuchen die besten auszuwählen und diese im professionellen Tonstudio endgültig zu produzieren.) Dabei ist nicht nur zu beachten, dass die richtige Option eindeutig zuzuordnen ist, sondern auch, dass keiner der Distraktoren zu leicht ausgeschlossen werden kann (s. o.). Dies wäre beispielsweise der Fall, wenn drei negative und eine positive Stimmung zu hören wären und letztere die richtige Lösung darstellte. Gleichzeitig dürfen aber auch nicht mehrere Optionen allzu ähnlich klingen, also etwa Varianten oder Abstufungen der weiter oben vorgestellten Prototypen im Sinne Neubers sein, da eine eindeutige Korrespondenz zwischen paraverbalem Signal und intendierter Bedeutung nicht existiert. Der beträchtliche Produktionsaufwand für diesen Itemtypus ist dadurch gerechtfertigt, dass hier keine Möglichkeit besteht, die richtige Lösung allein auf Grundlage der verbalen Information zu finden.

Typ 2: Eine geeignete Äußerung aus dem Originaltext wird in den Itemstamm hineingeschnitten und es werden vier mögliche Bedeutungen dieser Äußerung in Form verschiedener Anschlussätze angeboten. Dieser Typus lässt sich auch auf Basis eines bereits fertigen Hörtextes herstellen. Er eignet sich also auch beispielsweise für professionell produzierte Hörspiele, Radiosendungen etc. Wiederum sind auf der verbalen Ebene alle vier Optionen möglich, erst auf der paraverbalen Ebene entscheidet sich die Passung mit der intendierten Stimmung. Die Regieanweisungen für den Sprecher stehen kursiv in Klammern hinter der jeweiligen Option; sie erscheinen nicht im Testheft.

Der König sagt zum Leibarzt: „Der Prinz, mein geliebter Sohn, ist verschwunden.“ Was könnte er am ehesten anfügen?

A) „Machen wir uns auf die Suche.“ (fröhliche Aufforderung)

B) „Wenn ich ihn finde, hört er aber was.“ (schimpfend) 
$\square$ C) „Er wird wohl bald wieder auftauchen.“ (zufrieden, im Sinne von „halb so wild“)
D) „Ich mache mir große Sorgen um ihn.“ (besorgt wie im Itemstamm)

Bei diesem Itemtyp ist es möglich, die Formulierung des Itemstamms (außer der übernommenen Äußerung) und der Antwortoptionen von einem anderen Sprecher aufnehmen zu lassen.

Typ 3: Die einer Äußerung zuzuordnende Intention auf metasprachlicher Ebene wird expliziert. Auch dieser Itemtyp eignet sich für bereits fertiggestellte Hörtexte. Es ist allerdings zu beachten, dass die Formulierung der fraglichen Intention(en) in für Kinder verständlicher Weise möglich ist, was sich häufig als schwierig erweist. Ein Beispiel für diesen Typus ist das folgende:

Der König sagt: „Ich wünsche, dass das Schloss von oben bis unten durchsucht wird!“ Wie meint er das?
A) Das ist ein Befehl.
B) Das ist eine Bitte.
C) Das ist eine Entschuldigung.
D) Das ist eine Drohung.

Hier könnten theoretisch Wörter wie Befehl oder Drohung zu einem Wortschatzproblem führen - es wäre also denkbar, dass ein Kind zwar die Sprecherintention richtig erfasst, aber dennoch die falsche Option auswählt, weil es den treffenden Ausdruck nicht zuordnen kann. Diese Möglichkeit lässt sich nicht vollkommen ausschließen. Es wurde aber eine Variante erprobt, in der die gefragte Intention umschrieben wird wie in diesem Beispiel:

Die Köchin sagt: „Vielleicht ist er bloß weggelaufen. Kinder laufen manchmal von zu Hause weg." Weshalb sagt sie das so?

A) Sie weiß, dass der Prinz weggelaufen ist.

B) Sie will sich wichtig machen.

C) Sie möchte die anderen beruhigen.

D) Sie möchte endlich wieder ins Bett gehen.

Empirisch zeigt sich allerdings zwischen diesen beiden Typen - zumindest in dieser Aufgabe - kein Unterschied: Beide Items sind der Kompetenzstufe I zuzuordnen; das erste wird mit $89 \%$ noch etwas häufiger gelöst als das zweite (76\%). Eine systematische Analyse steht aber auch hier noch aus. 


\section{Zusammenfassung}

Im Projekt stịm·mig wurden Testaufgaben zur Messung der Zuhörfähigkeit von Grundschulkindern entwickelt und im Rahmen einer Großstudie evaluiert. Im Prozess der Entwicklung zeigte sich, dass zu allen Zeitpunkten mehrere Fachperspektiven in Betracht gezogen werden mussten, um zu (theoretisch) gelungenen Aufgabenformulierungen zu kommen. Diese Perspektiven wurden im Artikel anhand eines konkreten Aufgabenbeispiels dargelegt und sind unten in einer Tabelle (Tab. 1) stichpunktartig zusammengeführt. Ein wesentliches Charakteristikum solcher interdisziplinären Projekte ist, dass die Perspektiven nicht mehr einzelnen Personen(-gruppen) strikt zugeordnet werden können. Vielmehr ergibt sich im gemeinsamen Arbeitsprozess erkennbar eine gegenseitige Qualifikation aller Beteiligten. Dass solche Projekte vermutlich in Zukunft den Normalfall empirischer Forschungs- und Entwicklungsvorhaben im Feld der Deutschdidaktik darstellen, kann mithin nur als erfreulicher Umstand betrachtet werden, der zu einer weiteren Professionalisierung der Disziplin beitragen wird.

Tab. 1: Fachperspektiven im Projekt anhand eines konkreten Aufgabenbeispiels

\begin{tabular}{|c|c|c|c|c|}
\hline & $\begin{array}{l}\text { literaturwissen- } \\
\text { schaftliche und } \\
\text { sprachdidaktische } \\
\text { Perspektive }\end{array}$ & $\begin{array}{l}\text { psychometrische } \\
\text { Perspektive }\end{array}$ & $\begin{array}{l}\text { schauspieleri- } \\
\text { sche/ sprechwis- } \\
\text { senschaftliche } \\
\text { Perspektive }\end{array}$ & $\begin{array}{l}\text { administrativ- } \\
\text { technische } \\
\text { Perspektive }\end{array}$ \\
\hline Textauswahl & $\begin{array}{l}\text { Ist der Text inhaltlich } \\
\text { und sprachlich für die } \\
\text { Zielgruppe geeignet? }\end{array}$ & $\begin{array}{l}\text { Ist der Text für } \\
\text { alle Probanden } \\
\text { gleichermaßen } \\
\text { (un-) vertraut? }\end{array}$ & $\begin{array}{l}\text { Wie kann der Text } \\
\text { insgesamt ange- } \\
\text { messen inszeniert } \\
\text { werden (Grund- } \\
\text { stimmung, Figuren- } \\
\text { gestaltung)? }\end{array}$ & $\begin{array}{l}\text { Bestehen urhe- } \\
\text { berrechtliche } \\
\text { Bedenken? }\end{array}$ \\
\hline Textausschnitt & $\begin{array}{l}\text { Funktioniert der } \\
\text { gewählte Ausschnitt } \\
\text { im Sinne eines in } \\
\text { sich abgeschlossenen } \\
\text { Textes? }\end{array}$ & $\begin{array}{l}\text { Ist das Verhältnis } \\
\text { von Textlänge und } \\
\text { Zahl der Testitems } \\
\text { angemessen } \\
\text { (Testökonomie)? }\end{array}$ & $\begin{array}{l}\text { Wie viele und } \\
\text { welche Sprecher } \\
\text { werden benötigt } \\
\text { (Geschlecht, } \\
\text { Alter...)? }\end{array}$ & $\begin{array}{l}\text { Eigenprodukti- } \\
\text { on oder externe } \\
\text { Auftragsverga- } \\
\text { be? Vertragsge- } \\
\text { staltung? }\end{array}$ \\
\hline Testfragen & $\begin{array}{l}\text { Welche Informatio- } \\
\text { nen sind relevant für } \\
\text { ein angemessenes } \\
\text { Textverständnis? } \\
\text { Wie sind diese im } \\
\text { Gedächtnis repräsen- } \\
\text { tiert? }\end{array}$ & $\begin{array}{l}\text { Zielen die Fragen } \\
\text { auf unterschied- } \\
\text { liche Ebenen } \\
\text { des getesteten } \\
\text { Konstrukts ab } \\
\text { (verschiedene } \\
\text { Anforderungsbe- } \\
\text { reiche)? }\end{array}$ & $\begin{array}{l}\text { Wie un-/ähnlich } \\
\text { müssen bzw. dürfen } \\
\text { Antwortoptionen } \\
\text { klingen? Können } \\
\text { Emotionen und } \\
\text { Bedeutungen } \\
\text { stimmlich er- } \\
\text { kennbar markiert } \\
\text { werden? }\end{array}$ & $\begin{array}{l}\text { Können alle } \\
\text { Fragen vom } \\
\text { gleichen Spre- } \\
\text { cher eingespielt } \\
\text { werden? Sind } \\
\text { Schneidearbei- } \\
\text { ten im Tonstu- } \\
\text { dio erforderlich? }\end{array}$ \\
\hline
\end{tabular}




\begin{tabular}{|l|l|l|l|l|}
\hline & $\begin{array}{l}\text { literaturwissen- } \\
\text { schaftliche und } \\
\text { sprachdidaktische } \\
\text { Perspektive }\end{array}$ & $\begin{array}{l}\text { psychometrische } \\
\text { Perspektive }\end{array}$ & $\begin{array}{l}\text { schauspieleri- } \\
\text { sche/ sprechwis- } \\
\text { senschaftliche } \\
\text { Perspektive }\end{array}$ & $\begin{array}{l}\text { administrativ- } \\
\text { technische } \\
\text { Perspektive }\end{array}$ \\
\hline $\begin{array}{l}\text { Item-Kon- } \\
\text { struktion }\end{array}$ & $\begin{array}{l}\text { Welche Itemvarianten } \\
\text { (z. B. Modalität: nur } \\
\text { hören vs. hören + } \\
\text { mitlesen) sind zur } \\
\text { Hypothesenprüfung } \\
\text { nötig? }\end{array}$ & $\begin{array}{l}\text { Welche Item- } \\
\text { formate sollen } \\
\text { gewählt werden? } \\
\text { Formulierung } \\
\text { eindeutiger } \\
\text { Auswertungsan- } \\
\text { weisungen }\end{array}$ & - & $\begin{array}{l}\text { Wie aufwändig } \\
\text { ist die Auswer- } \\
\text { tung? Sind (ggf. } \\
\text { mehrere) Kodie- } \\
\text { rerschulungen } \\
\text { erforderlich? }\end{array}$ \\
\hline Testdesign & - & $\begin{array}{l}\text { "Rotierte } \\
\text { Anordnung der } \\
\text { Aufgaben/-blöcke } \\
\text { in Testheften (zur } \\
\text { Vermeidung von } \\
\text { Reihenfolgeef- } \\
\text { fekten) }\end{array}$ & $\begin{array}{l}\text { Stehen ausrei- } \\
\text { chend Test- } \\
\text { personen zur } \\
\text { Verfügung? Ist } \\
\text { die Stichprobe } \\
\text { repräsentativ? }\end{array}$ \\
\hline
\end{tabular}

\section{Literatur}

Baumert, Jürgen et al. (Hrsg.) (2001): PISA 2000. Basiskompetenzen von Schülerinnen und Schülern im internationalen Vergleich. Opladen: Leske + Budrich.

Behrens, Ulrike (2010): Aspekte eines Kompetenzmodells zum Zuhören und Möglichkeiten ihrer Testung. In: Bernius, Volker/Imhof, Margarete (Hrsg.): Zuhörkompetenz in Unterricht und Schule. Beiträge aus Wissenschaft und Praxis. Göttingen: Vandenhoeck \& Ruprecht, S. 31-50.

Behrens, Ulrike/Böhme, Katrin/Krelle, Michael (2009): Zuhören. Operationalisierung und fachdidaktische Implikationen. In: Granzer, Dietlinde/Köller, Olaf/ Bremerich-Vos, Albert (Hrsg.): Bildungsstandards Deutsch und Mathematik. Leistungsmessung in der Grundschule. Weinheim: Beltz, S. 357-376.

Böhme, Katrin (2011): Methodische und didaktische Überlegungen sowie empirische Befunde zur Erfassung sprachlicher Kompetenzen im Deutschen. Analysen zu den Bildungsstandards im Fach Deutsch für den Primarbereich. http:// edoc.hu-berlin.de/dissertationen/boehme-katrin-2011-12-16/PDF/boehme. pdf. Abgerufen am 05.10.2015.

Böhme, Katrin/Robitzsch, Alexander/Busè, Anne-Kathrin (2010): Zur Abgrenzung des Hörverstehens. In: Bernius, Volker/Imhof, Margarete (Hrsg.): Zuhörkompetenz in Unterricht und Schule. Göttingen: Vandenhoeck und Ruprecht, S. 81-104.

Bose, Ines et al. (Hrsg.) (2013): Einführung in die Sprechwissenschaft. Phonetik, Rhetorik, Sprechkunst. Tübingen: Narr Studienbücher.

Buck, Gary (2001): Assessing listening. Cambridge: Cambridge University Press. 
Fiehler, Reinhard (2014): „Von der Mündlichkeit zur Multimodalität ... und darüber hinaus." In: Grundler, Elke/Spiegel, Carmen (Hrsg.): Konzeptionen des Mündlichen. Wissenschaftliche Perspektiven und didaktische Konsequenzen. Bern: hep Verlag, S. 13-31.

Fiehler, Reinhard et al. (2004): Eigenschaften gesprochener Sprache. Theoretische und empirische Untersuchungen zur Spezifik mündlicher Kommunikation. Tübingen: Narr.

Grotjahn, Rüdiger (2000): Testen der Fertigkeit Hörverstehen. Leistungsmessung und Leistungsbeurteilung. http://www.unileipzig.de/herder/temp/lehrende/ tschirner/testen/hoeren.pdf. Abgerufen am 05.10.2014.

Imhof, Margarete (2003): Zuhören. Psychologische Aspekte auditiver Informationsverarbeitung. Göttingen: Vandenhoeck \& Ruprecht.

IQB (2013): Kompetenzstufenmodell zu den Bildungsstandards für das Fach Deutsch im Kompetenzbereich „Sprechen und Zuhören“ - Primarbereich Beschluss der Kultusministerkonferenz (KMK) vom 04.03.2010. Auf Grundlage des Ländervergleichs 2011 überarbeiteter Entwurf in der Version vom 13. Februar 2013. https://www.iqb.hu-berlin.de/bista/ksm/KSM_GS_ Deutsch_Z.pdf. Abgerufen am 05.10.2014.

Moosbrugger, Helfried/Kelava, Augustin (2012): Testtheorie und Fragebogenkonstruktion. Heidelberg: Springer Verlag.

Müller, Karla (2012): Hörtexte im Deutschunterricht. Poetische Texte hören und sprechen. Seelze: Klett Kallmeyer.

Neuber, Baldur (2002): Prosodische Formen in Funktion. Leistungen der Suprasegmentalia für das Verstehen, Behalten und die Bedeutungs(re)konstruktion. Frankfurt a. M.: Lang.

Nold, Günter/Rossa, Henning (2007): Hörverstehen. In: Beck, Bärbel/Klieme, Eckhard (Hrsg.): Sprachliche Kompetenzen. Konzepte und Messung. DESIStudie (Deutsch Englisch Schülerleistungen International). Weinheim: Beltz, S. 178-196.

Quasthoff, Uta (1980): Erzählen in Gesprächen. Tübingen: Narr.

Schnotz, Wolfgang (1996): Lesen als Textverarbeitung. In: Günther, Hartmut/ Ludwig, Otto (Hrsg.): Schrift und Schriftlichkeit. Writing and its Use. Ein interdisziplinäres Handbuch internationaler Forschung. Berlin/New York: de Gruyter, S. 972-982.

Zingg Stamm, Claudia/Käser-Leisibach, Ursula/Bertschin, Felix (2014): Ohrwärts. Zuhören und literarisches Hörverstehen. Kompetenzerhebung mit Förderangeboten für 9- bis 10-Jährige. Solothurn: Lehrmittelverlag Solothurn. 
Irene Pieper, Florentina Sâmihăian

\title{
International Research Cooperation in What Used to Be Called Mother Tongue Education. The Example of LiFT-2
}

\begin{abstract}
The paper explores the challenges and potentials of international research cooperation in the field of L1 (first language, language of schooling/of education, e. g. German in Germany, French in France; French, German, Italian and Romansh in Switzerland) by looking at general developments in L1-research since the 1980ies and at the project LiFT-2 as an example.
\end{abstract}

\section{Introduction}

While the history of research in Deutschdidaktik or Language and Literature Education is still young, international approaches in the field are even younger and certainly less expected than in other fields of research. The frame of reference of the discipline seems national rather than international: The discipline originates in a particular philology (German language and literature) and a particular school subject (German), and in both domains processes of language cultivation with regard to a national language or mother tongue were originally part of the program. Though the rise of the discipline in the second half of the $20^{\text {th }}$ century was no longer framed by an ideology of nation-building, Deutschdidaktik has since then focussed on the German language and literature, and the main protagonists would be experts of German as a specific language and of learning this language as mother tongue (for the history of the subject German see Roberg et al. 2010). International cooperation would be more or less restricted to cooperation of experts from German speaking countries and surroundings. However, for the last thirty to forty years researchers and practitioners in the field of language and literature education have put considerable effort into the internationalisation of the domains - at the beginning still labelled Mother Tongue Education (MTE). Before turning to specific networks, the general characteristics of such a cooperation need to be considered.

1) By definition various national, linguistic and cultural contexts are involved. Thus, diversity and plurality of contexts are at the bottom of every attempt to cross national or linguistic borders in the field (though not always acknowledged in the same way). 
2) Making reference to inter-national research cooperation in MTE is still unspecific in what concerns research approaches, disciplinary starting points and educational paradigms.

3) Agents of such cooperation are both researchers and practitioners, experts in teacher education at various stages and more or less close to the field of action (the actual teaching and learning in class). Their academic origins are manifold. Disciplines cover language and language education, literature and literature education, educational sciences, psychology, sociology and Fachdidaktik. A variety of academic cultures is involved. This becomes particularly evident with regard to Fachdidaktik which is a German concept that can also be found in Scandinavian contexts (as an export), but seldom anywhere else. ${ }^{1}$

4) The border between research and development is - like in Fachdidaktik in general - often not clear cut. Curriculum design and the design of tools that prove useful for teachers can be as much in focus as empirical research into language learning or the evaluation of interventions.

Protagonists of cooperation in the field of ,MTE' would generally share a common field of interest and a number of aims. However, they do not necessarily share a common style of thinking (Denkstil) in the sense of Ludwik Fleck (1935). Thus, there is a certain risk that understanding among agents is restricted by a somehow hidden factor, that is by differences in conceiving of the world they are not aware of.

The concept of Denkstil refers to the common ground a thought collective would share. The style of thinking is a result of a socialisation process and in continuous development, though not radically changing. It applies to the general approach to the world, to specific phenomena or to issues of a discipline and comes about by the circulation of thought and social practices. The frame of thought that is thus provided is not explicit, but powerful in conceiving of the world and can potentially enact constraints in understanding. At the same time, according to Fleck, the style of thinking forms the necessary basis of understanding. Shifts within a thought collective can lead to changes in the Denkstil and dynamics in a style of thinking are more likely where different thought collectives interact.

1 The term Fachdidaktik refers to an academic discipline which focuses on learning and teaching in a specific domain. Fachdidaktik Deutsch is thus linked to literary studies and linguistics on the one hand and to learning and teaching in literature and language on the other hand. As an academic discipline Fachdidaktik is concerned with theory and research while at the same time aiming at improving teaching and learning in the field. 
Following Ludwik Fleck, there is thus a considerable risk that thorough understanding cannot be achieved. However, it can be argued that there is also a considerable potential for making the implicit explicit and for clarifying and developing approaches to research and practice.

In our experience, international encounters between researchers and other experts in the field not necessarily (or even: seldom) start by clarifying the various backgrounds of the protagonists which makes it likely that the general frame of thinking is even more implicit than in interdisciplinary research where the disciplinary anchors are given explictly right from the beginning or form the basis of cooperation. This is somehow balanced by the shared aims. However, it should be acknowledged that the clarification of what to assess and/or what to explore is all the more necessary the broader the scope of agents and contexts.

\subsection{Two Networks: IAIMTE and IMEN}

All in all, international cooperation in mother tongue education (MTE) or first, second and standard languages as languages of schooling (L1) has an epistemic potential. In a way, this is the experience of members of the two central associations resp. networks that have promoted this cooperation in the past: the International Association for the Improvement of Mother Tongue Education, IAIMTE (now: ARLE), and the International Mother Tongue Education Network, IMEN. By briefly describing aim and scope of these two networks we aim at demonstrating recent developments in what concerns international cooperation in MTE and L1. Both networks also served as a platform for the form of international cooperation that came into practice in the LiFT-2-project.

The International Mother Tongue Education Network, IMEN, mainly worked between 1984 and 2007. Here, international cooperation starts from the acknowledgement of difference in the various national contexts. The epistemic element is particularly strong. Protagonists aim at comparative approaches in order to explore and specify diversity in the field of MTE. Comparison in IMEN is the path to gaining deeper knowledge of MTE in its various forms, on a methodological level the empirical approaches are close to ethnography (cf. Herrlitz et al. 2007, the editors being key protagonists of the IMEN). The work is close to the field (e. g. classroom visits by multi-national teams, close cooperation with teachers). Besides curriculum research was carried out. Though the network no longer stages conferences or other activities the approaches developed by IMEN are still present in current research (e. g. Doecke/van de Ven 2011).

The International Association for the Improvement of Mother Tongue Education, IAIMTE, was founded by Gert Rijlaarsdam and Ken Watson in 1996. It has 
been transferred into the International Association for Research in L1 Education (languages, literatures, literacies), the ARLE, in 2014. International cooperation in the IAIMTE is based on the conviction that it is beneficial for MTE to share expertise and to develop a common knowledge rather than work on the same or on similar issues in ,splendid isolation. It is generally acknowledged that there is linguistic, cultural and educational diversity and there is room for exploring these differences, too. But the main aim is to allow for exchanges in research and development in a field where, this is a central assumption, researchers and practitioners from various places of the world can benefit from each other's work and make progress by cooperation. Thus, there is no common methodology, but a common concern. The mission statement of the IAIMTE reads accordingly:

„Divided as we are by our distinct languages and cultures, we do share a common concern: the quality of the teaching and learning of mother tongues. Throughout the world, education systems are confronted with major challenges. Societal demands for literacy and communicative competence are growing and at the same time, there is an increase in the cultural and linguistic heterogeneity of school populations. In response to these trends, promising research and development in the learning and teaching of mother tongues is done in many countries ... in splendid isolation. A pity, for national work in the improvement of language education can profit enormously from international exchange. This, in a nutshell, is the mission of the IAIMTE: the International Association for the Improvement of Mother Tongue Education." (cf. http://www.iaimte.com/mission.asp)

\subsection{From IAIMTE to ARLE and from Mother Tongue Education to L1 Education}

The IAIMTE has been continuously active with international conferences every two years, several Special Interest Groups and frequent seminars in different places of the world. Though with a particularly strong basis in Europe, the IAIMTE also goes overseas (e. g. to Hong Kong in 2014) and has active members in all continents. Besides, it provides an international peer reviewed journal since 2000, which is published online with free access and offers a platform for communicating research contributions from the various domains within language and literature education: 'L1 Educational Studies for Language and Literature' (http://www.l1research.org). The network has been growing ever since it came into existence and the conferences have continuously been attracting more and more delegates. A general tendency to internationalisation in educational research and development, the corresponding university policies and international research funding programs by the EU and other bodies probably have supported these developments. 
On an informal level, members appreciate the opportunities for encounters of various sorts within the network and its general openness, not always characteristic for academic life. In order to further strengthen the association, its visibility and scope of action, it was decided by the members in Paris 2013 to formally establish the association and at the same time adapt its name and mission to meet developments since the late 1990ies. The new name thus shows the shift from the concept of mother tongue to a concept of language learning that takes into account the variety of linguistic backgrounds in today's classrooms and the diversity of situations regarding the languages of schooling. L1, which is also the acronym of the journal, refers to the language of schooling, which often is the learner's first language, but increasingly a second language or one among different languages available (cf. the explanation of the concept at http://www.l1 research.org).

We thus argue that the shift towards L1 education characterises the current situation with regard to what used to be mother tongue education: The growing awareness of plurality on both the national and international level calls for a consideration of languages, literatures and literacies. Key concepts are no longer linked to the homogeneous notion of mother tongue, but rather to diversity, to plurilingual and intercultural education and to the languages of schooling, as it is reflected by the project Languages in Education, Languages for Education. A platform of resources and references in plurilingual and intercultural education by the Language Policy Unit of the Council of Europe (cf. Council of Europe/Language Policy Unit 2006-2016). Currently, the common emphasis is indeed on sharing insights into the various fields of practice (which share an inert diversity), but also on communicating research and development in order to benefit as best we can on what has already been achieved. An example in this respect is the project LiFT-2.

\section{LiFT-2}

LiFT-2 was funded by the EU-Comenius-Program between 2009 and 2012. The aim of the project was to design a Literary Framework for Teachers in Secondary Education, LiFT-2. ${ }^{2}$ The Framework is meant to assist teachers in ensuring progression in literary reading with secondary students. It focusses on narrative texts (novels and extensive/longer narratives, e.g. novelas). As an international framework it potentially reaches out to teachers in many European countries so that the insights and tools on offer can be shared and used not only on a national

2 The project is described more extensively in Pieper (2014). 
level, but across language borders. Besides, LiFT-2 encourages a broader notion of literature teaching, since it refers to national, international and world literature.

\subsection{The project in general}

Key principles of LiFT-2 are to be close to praxis with all the instruments it has been providing and to be accessed easily, namely via a website. In the project, researchers and teacher educators from six European countries worked together and finally drafted the Framework that has been available since $2012^{3}$ : from the Netherlands (Theo Witte, University of Groningen, project leader), from Romania (Florentina Sâmihăian, University of Bucharest), from Germany (Irene Pieper, University of Hildesheim), Finland (Raisa Simola, University of Eastern Finland in Joensuu), Portugal (Regina Duarte, University of Braga) and Czech Republic (Stepanka Klumparova, University of Prague). An implicit assumption of the endeavour probably was that the project partners, all experts in literature education, would be sharing a common style of thinking - at least to such an extent that the Framework would be achievable. Three key concerns are at the heart of LiFT-2:

1) The main aim was to develop a framework that helps to describe and to moderate progression in literary reading. It should answer the question how to lift students from a certain level of literary development to another in a way which is close to literary praxis in the various classrooms.

2) Texts and their characteristics should be related to learners and their characteristics. One aim is indeed to provide suggestions of literary texts suitable for specific levels of the learners.

3) Texts should also be addressed with regard to their learning potential. Thus, we intend to offer instruments that help to describe challenges a text might provide in the light of their potential to progression in literary reading.

The project did not explicitly focus on the potential challenges of achieving these aims in an international context. It was not part of the program to explore literature and literature teaching as culturally bound.

In order to meet the concerns just spelt out the project worked on three keyquestions:

1) What can be considered as typical for learners of a specific stage of schooling/ school-age in the field of reading narratives?

3 We here name the project-leader and the responsible project-partners in the partnercountries. The teams often involved more experts. This can be traced on the websites of the partners, to be accessed via: http://www.literaryframework.eu. (22.01.2016). 
LiFT-2 distinguishes four to six levels of literary development.

2) What can be considered as typical for the texts that seem adequate for the respective levels?

LiFT-2 recommends particular texts for particular levels.

3) How can progression be designed in such a way that learners can reach the next level?

LiFT-2 has designed transitions that should help teachers to ensure literary development in the context of education.

It could be argued that the project thus works on the most challenging topics of research in literature education. The empirical basis of describing (and modelling) progression in literary reading has long been considered as weak. Thorough models of competence in this field are not available. Both the richness of literary texts and the individuality of interpretative processes seem to hinder. On the other hand it is part of the teaching of literature to ensure progression with students and project-partners would argue that there is a need for an instrument that assists teachers in designing the learning with literature in a developmental perspective.

The project could go back to a model that was designed by project-leader Theo Witte. In his dissertation Witte had designed a system of levels for teachers in upper secondary education (Witte 2008). His starting point was the introduction of a portfolio-approach in the Dutch literature classroom of Upper Secondary. According to this approach, students should set up their individual reading list by choosing from a list of texts. They should perform tasks individually and hand in a portfolio to their teacher. Witte observed a need of support for both learners and teachers in making adequate choices, perform tasks and ensure progression. In order to describe students' development and also provide an instrument that would assist the teachers he drew on the pedagogical content knowledge of the teachers (cf. Shulman 1986). Via work group discussions and focus group discussions with the teachers (cf. Witte et al. 2012) he aimed at making their implicit knowledge on learner characteristics and book features explicit. The data collected via these discussions at the same time made it possible to stay close to the teachers' language when designing the levels of literary development. These levels by now structure Dutch literature education to a large extent. ${ }^{4}$

4 See the website http://www.lezenvoordelijst.nl/colofon/ (22.01.2016). The website constantly offers new recommendations of books. The system of levels also often structures the way books are arranged in school libraries. Learners are encouraged to monitor their own development by moving up in their choices and their performance. For a more 
Thus, LiFT-2 serves as an example of how to profit from international exchange in $\mathrm{L} 1$ education. The project team adapted a model which had proved sensible in a national context, the assumption being that such a transfer is possible despite the differences in the various countries, the educational systems and potentially in the style of thinking, too. Evidence for a common basis could be found in the work of the language policy division of the Council of Europe which refers to a shared understanding of literature education (see Aase et al. 2007) and in a curriculum comparison which was carried out in LiFT-2 (see 2.2). Besides, the procedures of the project were meant to ensure that project results could be shared and offered room for adaptations (see below and point 2.3). ${ }^{5}$ To explore the differences in the process of development and implementation was, however, not an aim in itself and was beyond the scope of the project and its funding. However, traces of tensions and differences were discovered in the course of the project and should be followed up (see below and part 3).

Under the presupposition that there is a sound common basis it seems most sensible to adapt an existing model in an international context. On the level of improving education this is both practical and economical. Still, it may be considered problematic in the frame of research as quality here is connected to originality and innovation (cf. Wiemer 2011). There is some innovation in LiFT-2, though, since it extended the Dutch model designed by Witte and provided new perspectives for the research community and the community of practitioners: LiFT-2 covers both lower and upper secondary education, starting at the age of $12 .{ }^{6}$ Hence, the impact of development of the learners is stronger. Besides, LiFT-2 puts more focus on designing progression via teaching in class. The model is thus moving beyond its usability in a portfolio-approach that focusses on individual reading processes and meets the different teaching cultures. The model of transitions is an original contribution of LiFT-2. The third aspect concerns the reading lists and recommendations: One aim of LiFT-2 is to balance the national reading lists (more or less canonical and more or less fixed) with an international choice of text that covers world literature, too. LiFT-2 thus aims at contributing to intercultural understanding via a more varied choice of texts. A fourth aspect concerns curriculum research: in order to ensure a common basis for the work of the partners and the design of a European framework the curriculum comparison mentioned above

extensive account of the 'empirically grounded theory of literary development' in English see Witte et al. (2012).

5 It would also be possible to consider it as a preliminary task.

6 School systems vary considerably in Europe with regard to forms of schooling and grades in relation to age. 
was carried out and published for an international audience (Witte/Sâmihăian 2013). Before turning to the empirical part and the main procedures of the project we describe the comparative analysis.

\subsection{A comparative analysis of the formal literature curriculum in six European countries ${ }^{7}$}

\subsubsection{Aims, points of reference and methodology of the research}

The comparison of eight European literary curricula aimed at: identifying the theories behind the curricula in different countries; comparing the ways in which a developmental line is present in the analysed curricula; ensuring a common basis for the framework (by identifying the curricular perspective on the three dimensions on which the LiFT-2 framework is built: students, books and didactics); becoming aware of the differences and specificities of studying literature in the six European countries that participated in the project.

The first point of reference in our comparative analysis consisted of the four paradigms of studying literature we tried to identify in the analysed curricula. These paradigms are (Ongstad et al. 2004; Rijlaarsdam/Janssen 1996; Sawyer/Van de Ven 2007; Verborrd 2005; Witte et al. 2006):

1) the cultural model which emphasizes the idea that literature is part of the cultural development and stresses enrichment of students' cultural knowledge

2) the linguistic model which focuses on text's autonomy and on developing students' analytic skills of interpreting a text

3) the social model which states that literature is part of reality, offering different perspectives, and that students should be encouraged to develop their social awareness and critical thinking through reading and discussing what they read

4) the personal growth model/reader oriented model which focuses on the impact literature has on readers; as a consequence, students are encouraged to give personal responses to the text they read and to clarify their own values in confrontation with those induced by a literary text.

A more comprehensive presentation of these four models is presented in the following table:

7 This part takes up central insights spelt out in the article by Witte/Sâmihăian (2013). 
Tab. 1: Curricular aspects of four paradigms of teaching literature ${ }^{8}$

\begin{tabular}{|c|c|c|c|c|}
\hline Aspects & Cultural & Linguistic & Social & $\begin{array}{l}\text { Personal } \\
\text { growth }\end{array}$ \\
\hline $\begin{array}{l}\text { 1. Competences } \\
\text { developed through } \\
\text { literature teaching }\end{array}$ & cultural literacy & $\begin{array}{l}\text { aesthetic awa- } \\
\text { reness }\end{array}$ & social awareness & $\begin{array}{l}\text { personal deve- } \\
\text { lopment }\end{array}$ \\
\hline 2. Content & $\begin{array}{l}\text { literary history, } \\
\text { literary move- } \\
\text { ments, } \\
\text { (other arts) }\end{array}$ & $\begin{array}{l}\text { literary theory, } \\
\text { style, } \\
\text { text structure and } \\
\text { meaning } \\
\text { (other arts) }\end{array}$ & $\begin{array}{l}\text { ethical, social, } \\
\text { political issues, } \\
\text { reader response, } \\
\text { student perceptions }\end{array}$ & $\begin{array}{l}\text { personal expe- } \\
\text { rience, student } \\
\text { perceptions, } \\
\text { reader res- } \\
\text { ponses } \\
\text { (other arts) }\end{array}$ \\
\hline 3. Approach to texts & $\begin{array}{l}\text { literary context } \\
\text { (biography, } \\
\text { epochs) }\end{array}$ & $\begin{array}{l}\text { mostly formal } \\
\text { aspects of texts - } \\
\text { text analysis }\end{array}$ & $\begin{array}{l}\text { non-literary } \\
\text { context, reader } \\
\text { responses }\end{array}$ & $\begin{array}{l}\text { reader res- } \\
\text { ponses }\end{array}$ \\
\hline $\begin{array}{l}\text { 4. Text selection } \\
\text { criteria }\end{array}$ & national canon & $\begin{array}{l}\text { acknowledged } \\
\text { aesthetic values }\end{array}$ & $\begin{array}{l}\text { topics relevant for } \\
\text { age group }\end{array}$ & $\begin{array}{l}\text { student pre- } \\
\text { ferences and } \\
\text { interests }\end{array}$ \\
\hline 5. Class management & $\begin{array}{l}\text { listening to } \\
\text { lectures }\end{array}$ & $\begin{array}{l}\text { whole-class dis- } \\
\text { cussion, writing }\end{array}$ & $\begin{array}{l}\text { whole-class discus- } \\
\text { sion, } \\
\text { peer discussion }\end{array}$ & peer discussion \\
\hline 6. Teacher role & $\begin{array}{l}\text { Expert who deli- } \\
\text { vers information }\end{array}$ & $\begin{array}{l}\text { expert, modelling } \\
\text { literary analysis }\end{array}$ & $\begin{array}{l}\text { Leader of the } \\
\text { discussion in the } \\
\text { classroom }\end{array}$ & $\begin{array}{l}\text { guide, facilita- } \\
\text { tor, stimulator }\end{array}$ \\
\hline 7. Assessment & $\begin{array}{l}\text { reproduction of } \\
\text { knowledge about } \\
\text { text and context } \\
\text { (cultural epochs) }\end{array}$ & $\begin{array}{l}\text { skills of text } \\
\text { analysis }\end{array}$ & $\begin{array}{l}\text { Comprehension/ } \\
\text { interpretation skills } \\
\text { applied to social } \\
\text { values }\end{array}$ & $\begin{array}{l}\text { Compre- } \\
\text { hension/ } \\
\text { interpretation } \\
\text { skills applied } \\
\text { to personal } \\
\text { values }\end{array}$ \\
\hline & \multicolumn{2}{|l|}{ content-oriented } & \multicolumn{2}{|l|}{ student-oriented } \\
\hline
\end{tabular}

The above description of the four paradigms of studying literature is a theoretical one, which can take different shapes in nowadays practices, as teachers have to adjust their strategies to a generation of students labeled as 'homo zappiens' or 'digital natives' (Prensky 2010; Veen/Vrakking 2007). From this point of view, even if in a curriculum the dominant paradigm is the cultural or the linguistic one, the approaches need to be extended to or combined with methods derived from the social and personal growth model; also the teachers' role, classroom

8 Adapted from Ongstad et al. (2004), Verboord (2005), Rijlaarsdam/Janssen (1996), Sawyer/Van de Ven (2007) and Witte et al. (2006). For the notion of competences see 2.2.3.1. 
management or assessment strategies will be informed by the social and personal growth models. If the dominant model is social or personal growth, then teachers may feel the need to make some extensions in what aims of teaching and content are concerned.

The second point of reference was a line of development of the literary competences (Witte et al. 2012), which we built based on the features of the four paradigms and also on the idea of the continuity and accumulation of paradigms, with shifting dominants, starting from a naïve dependent reader (low literary competence) who can become, at the end of the road, a sophisticated autonomous reader (high literary competence). The sense of this developmental model is presented in Figure 1.

Fig. 1: The cumulative paradigms of teaching literature in the literary framework

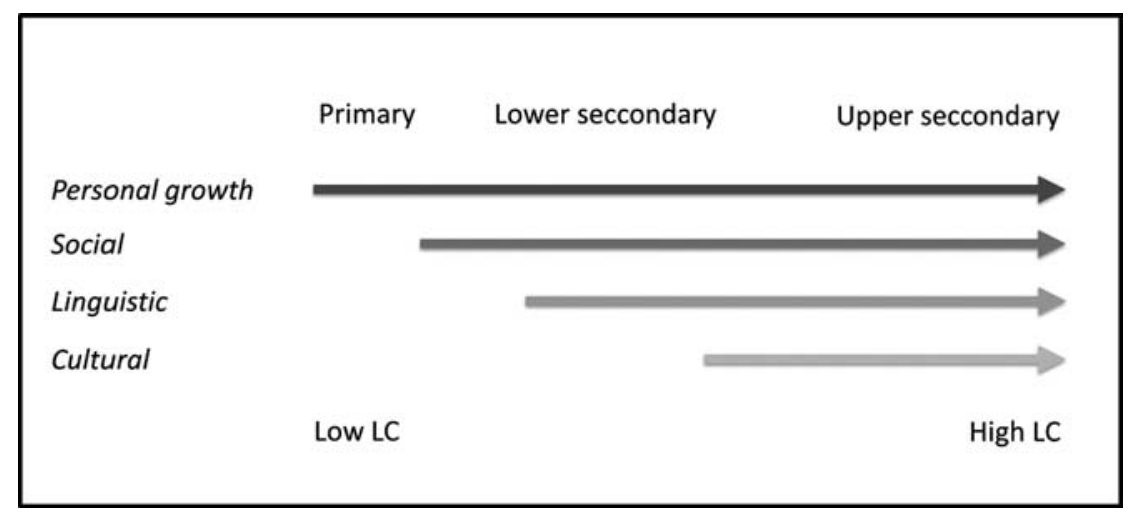

In our study, we compared this cumulative model of literary paradigms with the line of evolution in the formal curricula of the six countries. We tried to find answers to the following questions:

(1) What are the dominant paradigms in teaching literature in Europe, bearing in mind students, books and didactics?

(2) How do the curricula relate to the development-oriented framework?

Our study is a descriptive one and followed three steps: collection of data regarding the documents of formal curricula for literature in each country; grouping the data for comparison, according to the four paradigms of studying literature; presenting and evaluating the results. The members from our project team served as experts for their respective curricula - available in the respective language and provided the group with the necessary information. A guideline was used 
to collect the data. The information was shared in our project meetings so that a common understanding could be achieved.

The researched documents were the official curricula for secondary education in use at the time of research (2009-2012) in six European countries participating in the project: Czech Republic, Germany, Finland, The Netherlands, Portugal and Romania. For Germany, where each land has its own educational policy and curriculum, the experts for this country chose three lands they considered representative for different ways of approaching literature: Bavaria, Lower Saxony, Thuringia. ${ }^{9}$ We are aware that we missed the actual practices in school, but our research could not be extended to that dynamic reality.

We analysed the data provided by each country, focusing on the three dimensions that articulated the LiFT-2 framework: students, books, and didactics.

\subsubsection{Results of literary curriculum comparison}

\subsubsection{The first dimension of the comparison: students' literary competences}

The first dimension refers to the expected learning outcomes of students for studying literature (the first criteria in Table 1). We noticed that the understanding of curricular concepts like 'goals', 'aims', 'attainment targets' and 'competences' is different in the curricula we compared. As we are working in a European context we prefered to make use of the umbrella term 'competences' as defined in The European Framework for Key Competences for Lifelong Learning: "a combination of knowledge, skills and attitudes appropriate to the context" (European Commission 2006, p. L 394/13). Our analysis aimed both at comparing the competences identified in the curricula for a certain grade (a horizontal perspective) in order to see the dominant models and at comparing the lines of progression in using the four models of studying literature along secondary education (a vertical perspective). For this last purpose, we compared curricula for grade 7 and 12.

We registered almost all ${ }^{10}$ the competences phrased in the curricula of the respective country and associated them to one or more of the four paradigms

9 Bavaria is considered a traditionally conservative 'land' in the South, as conservative politics have always influenced their educational policies; Lower Saxony is a 'land' which has always seen more political change and whose curricula have been shaped by policies of the Social Democrats and their respective partners more strongly; and Thuringia is seen as a new 'land' with possible traces of East-German traditions, but open to various developments. The curricula covered are those of the Gymnasium.

10 There are a few competences we could not classify because they were not clear or relevant for our literary perspective. 
in Table 1. We are aware that using numbers to express the relative dominance of competences in a curriculum can result in some complications, not the least because the differences between curricula, their structure and their wording made the comparison challenging. Nevertheless, we chose this way because we assume that the extensity to which competences associated with a model are present in a curriculum is an indication of the dominance of a certain model. The results of this classification are presented in Table 2.

Tab. 2: Literary competences in six European countries associated with the four models of studying literature, grade 7 (in brackets: number of competences)

\begin{tabular}{|l|c|c|c|c|l|}
\hline Country Paradigm & Cultural & Linguistic & Social & $\begin{array}{c}\text { Personal } \\
\text { growth }\end{array}$ & $\begin{array}{l}\text { Country } \\
\text { paradigm } \\
\text { dominance }\end{array}$ \\
\hline Czech Republic (Cz) & $23 \%(3)$ & $46 \%(6)$ & - & $31 \%(4)$ & linguistic \\
\hline Finland (Fi) & - & $36 \%(4)$ & $28 \%(3)$ & $36 \%(4)$ & $\begin{array}{l}\text { linguistic, } \\
\text { personal }\end{array}$ \\
\hline $\begin{array}{l}\text { Germany- Bavaria } \\
\text { (D-Bav) }\end{array}$ & $22 \%(2)$ & $34 \%(3)$ & $22 \%(2)$ & $22 \%(2)$ & linguistic \\
\hline $\begin{array}{l}\text { Germany - Lower } \\
\text { Saxony (D- LS) }\end{array}$ & $14 \%(1)$ & $29 \%(2)$ & $14 \%(1)$ & $43 \%(3)$ & personal \\
\hline $\begin{array}{l}\text { Germany - Thuringia } \\
\text { (D-Th] }\end{array}$ & - & $56 \%(5)$ & $33 \%(3)$ & $11 \%(1)$ & linguistic \\
\hline Netherlands (Nl) & - & $22 \%(2)$ & - & $78 \%(7)$ & personal \\
\hline Portugal (Pt) & $12 \%(3)$ & $36 \%(9)$ & $24 \%(6)$ & $28 \%(7)$ & linguistic \\
\hline $\begin{array}{l}\text { Romania (Ro) } \\
\text { Overall paradigm }\end{array}$ & - & $43 \%(3)$ & $14 \%(1)$ & $43 \%(3)$ & $\begin{array}{l}\text { linguistic, } \\
\text { personal }\end{array}$ \\
\hline Iominance & I & III & II & linguistic \\
\hline
\end{tabular}

The comparative analysis of the literary competences shows interesting results about the presence of the four models in the formal curriculum for grade 7 . The first aspect to be mentioned is that all curricula are poly-paradigmatic, and we can see a combination of content-oriented and student-oriented paradigms. We can see clear differences between the eight European curricula for grade 7 if we take into consideration the dominant paradigm. The cultural model is relatively powerful in the curricula of $\mathrm{Cz}$ and $\mathrm{D}$-Bav; the linguistic model reaches its highest percentage in the D-Th curriculum, but is also clearly present in the curricula of $\mathrm{Cz}$ and Ro; the social model is more powerful in D-Th and Fi than in the other European curricula; the personal growth model has its strongest position in the $\mathrm{Nl}$ curriculum, 
at considerable distance ahead of D-LS, Ro, and Fi, where this paradigm has also a strong position.

To better understand our approach and the spirit of the analysed curricula, we present some relevant examples of competences for each model, choosing the countries where these models had the best percentages:

- for the cultural model, we identified in the Cz curriculum the following competences: list the basic literary styles and their significant representatives in Czech and world literature; recognise the basic literary styles and genres; compare them and their function; list their major representatives;

- for the linguistic model, the curriculum of G-Th mentions competences such as: identifying literary texts/text components; analysing and interpreting selected literary text passages; analysing the value of narration in epic texts; deepening the knowledge of linguistic features of poetic texts, developing an awareness of the lyrical ' $I$ ';

- for the social model, the curriculum of G-Th has also the highest percentage of competences: comprehension of the values of characters, their behaviour and the motives and intentions in epic and dramatic text; dialogic and scenic reading of dramatic texts; deepening the ability of watching and listening, especially while dealing with dramatic texts; understanding dramatic conflicts;

- for the personal development model, the $\mathrm{Nl}$ curriculum provides the most numerous competences: relate the story to their own world; give re-creative response (e. g. drawing), give personal (subjective) response, use emotive criteria (e. g. exciting, sad, stupid); express their own antipathy or sympathy for characters and their experiences.

To conclude, we can say that the linguistic paradigm is dominant in grade 7 , occupying first place in the curricula of $\mathrm{Cz}, \mathrm{Fi}, \mathrm{Bav}$, Th, Pt and Ro ( 6 of the 8 curricula examined). Second place is taken by the personal growth paradigm that is powerful in Nl, Fi, LS and Ro. Third place is occupied by the social model, and last place is taken by the cultural paradigm, as expected for this level of schooling.

As we need to compare the expectations related to studying literature at different stages of schooling, we analysed, by using the same methodology, the curricula for grade 12 , the end of secondary education. The results of this analysis are presented in Table 3. 
Tab. 3: Literary competences in six European countries associated with the four models of studying literature, grade 12 (in brackets: number of competences)

\begin{tabular}{|c|c|c|c|c|c|}
\hline Country Paradigm & Cultural & Linguistic & Social & $\begin{array}{c}\text { Personal } \\
\text { growth }\end{array}$ & \begin{tabular}{|l|} 
Country \\
paradigm \\
dominance \\
\end{tabular} \\
\hline Czech Republic (Cz) & $23 \%(4)$ & $53 \%(9)$ & $12 \%(2)$ & $12 \%(2)$ & linguistic \\
\hline Finland (Fi) & $58 \%(4)$ & $14 \%(1)$ & $14 \%(1)$ & $14 \%(1)$ & cultural \\
\hline $\begin{array}{l}\text { Germany- Bavaria } \\
\text { (D-Bav) }\end{array}$ & $50 \%(1)$ & $50 \%(1)$ & - & - & $\begin{array}{l}\text { cultural, } \\
\text { linguistic }\end{array}$ \\
\hline $\begin{array}{l}\text { Germany - Lower } \\
\text { Saxony (D- LS) }\end{array}$ & $36 \%(4)$ & $36 \%(4)$ & $19 \%(2)$ & $9 \%(1)$ & $\begin{array}{l}\text { cultural, } \\
\text { linguistic }\end{array}$ \\
\hline $\begin{array}{l}\text { Germany - Thuringia } \\
\text { (D-Th] }\end{array}$ & $44 \%(10)$ & $26 \%(6)$ & $17 \%(4)$ & $13 \%(3)$ & cultural \\
\hline Netherlands (Nl) & $19 \%(3)$ & $31 \%(5)$ & $25 \%(4)$ & $25 \%(4)$ & linguistic \\
\hline Portugal (Pt) & $12 \%(1)$ & $60 \%(7)$ & $7 \%$ & $14 \%(6)$ & linguistic \\
\hline Romania (Ro) & $49 \%(4)$ & $13 \%(1)$ & $25 \%(2)$ & $13 \%(1)$ & cultural \\
\hline $\begin{array}{l}\text { Overall paradigm } \\
\text { Dominance }\end{array}$ & II & I & III & IV & linguistic \\
\hline
\end{tabular}

A common feature of the curricula for grades 7 and 12 is that they are both poly-paradigmatic. But the difference is in the predominance of content-oriented paradigms in the curricula for grade 12. Comparing the position of each model in all the examined curricula, we notice that the cultural paradigm has the highest percentage in the curriculum of $\mathrm{Fi}$, but it has also a strong position in D-Bav, $\mathrm{D}$-Th and Ro. The linguistic paradigm is dominant in the formal curriculum of $\mathrm{Cz}$, with the highest percentage at European level also. In D-Bav, D-LS, Nl and Pt, the linguistic paradigm shares its powerful position with other paradigms. The social model is most clearly visible in the curricula of $\mathrm{Nl}$ and $\mathrm{Ro}$. The personal model, finally, has a remarkable position in Pt only at this stage of schooling. With the exception of the Pt curriculum, and to some extent also the curricula of $\mathrm{Nl}$ and Ro, the personal and social paradigms seem to play a minor role in grade 12 . All in all, the linguistic paradigm has the most powerful influence in grade 7 as well as in grade 12 .

We present here some of the competences in the curricula for grade 12 we associated with the cultural model (that has its peaks in Fi curriculum) and with the personal development model (at its best in the $\mathrm{Nl}$ curriculum):

- for the cultural model, we identified the following competences in the Fi curriculum: read text in oral and written tradition - from folklore to literature; 
understand the significance of language and literature in the construction of a national identity; place Finnish literature in its temporal and cultural contexts; present key literary works and themes;

- for the personal development model, the $\mathrm{Nl}$ curriculum, consistent with emphasizing the role of this model along the school years, offers the most numerous competences: identify oneself emphatically with different characters; use emotive criteria; motivate their interest in some authors.

In Figure 2 we synthetise the average evolution of the four paradigms at a European level (based on the eight literary curricula we analysed), so that we can see what is the effect of dominance of paradigms on the idea of cumulative or shifting paradigms in a developmental way, as presented in Figure 1.

Fig. 2: Comparative overview of the literary paradigms in grades 7 and 12

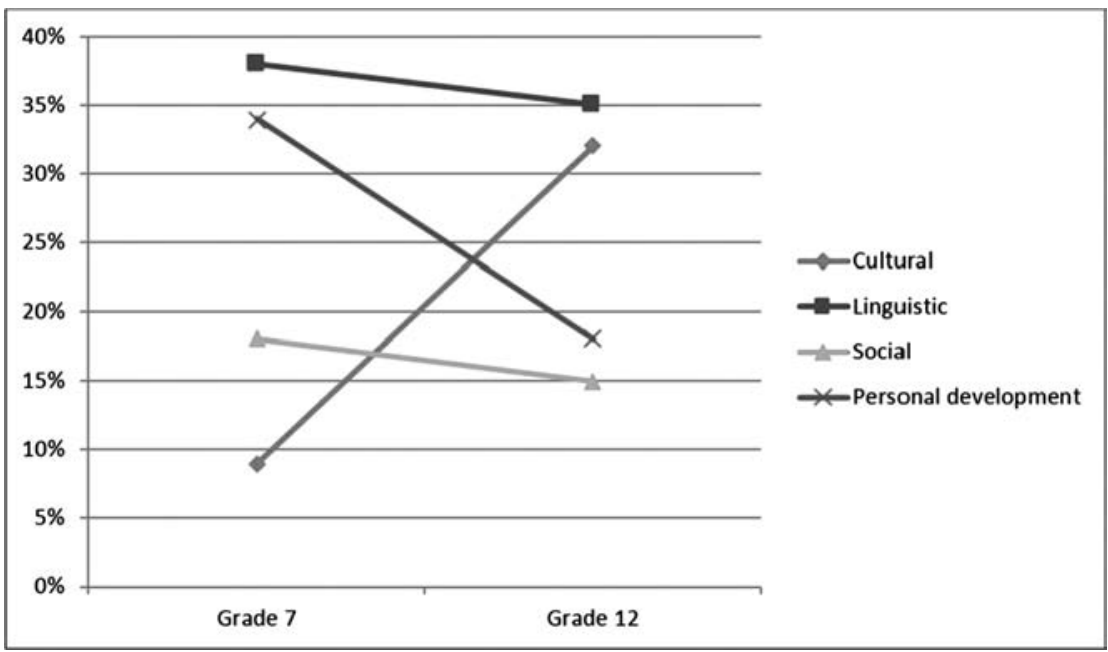

In the above figure, it is interesting to notice the spectacular changes of influence that the cultural and personal development models undertakes over the grades. Like in a mirror, the cultural model grows from grade 7 to 12 , while the personal development model decreases from grade 7 to 12 . In all curricula in grade 12, we see that the importance of content-oriented literature education has increased ( $48 \%$ to $67 \%$ ), and the cultural domain especially has more influence in upper secondary ( $9 \%$ to $32 \%$ ). This is at the expense of the personal domain, which greatly declined in importance (34\% to $18 \%$ ). The social domain in grade 7 holds a modest position and this remains so in grade $12(18 \%$ to $15 \%)$. However, the 
linguistic domain holds a dominant position in grade 7 and maintains that position in grade 12 (38\% to $35 \%)$.

These results show that the majority (five of eight countries/states) of the analysed curricula follow the developmental line of the paradigms presented in Figure 1, evolving from a more student-centred (personal/social) curriculum in grade 7 to a more content-centred one (linguistical/cultural) in grade 12. With regard to our international project, this seems to indicate some common ground, but also some variety in what concerns priorities in literature teaching.

\subsubsection{The second dimension of the comparison: texts recommended for study}

The second dimension concerns the type of books/texts or criteria for selecting books, and also content referring to literary genres, epochs, cultural movements or topics (as they can be linked to a certain type of literary text), all mentioned in the recommendations of the formal curriculum. Each country has a view about when to introduce the canon (for the actual debates on the importance of canon in literary education, see also Fleming 2010; Pieper 2006; Sâmihăian 2007) and what is worth being studied. To reflect this, our analysis also included a perspective on the literary canon in the curricula compared because we intended to include an international list of books in the Framework (http://www.literaryframework.eu/).

For comparing the books recommended for study in the six countries, we had in view what curricula say about text selection criteria and content.

It was quite difficult to compare the aspects regarding this dimension, because the eight curricula addressed this issue differently. Some curricula refer to authors or titles, others to periods or literary genres; others mention themes or a minimum number of texts to be read. In some curricula the criteria for text selection are not clearly defined, but they are implicit. They take into consideration either the books' characteristics or a guiding principle for selecting the books, like the accessibility for young learners. In one case (Portugal) some titles are compulsory. We also had in view the different ways of approaching the national and the universal canon. ${ }^{11}$

As expected, the books recommended by the curricula for grade 7 fit more the personal development paradigm. Only Czech Republic and Portugal diverge in this respect. The three German Länder follow more eclectic criteria. For grade 7 it is interesting to note the distribution of classic and contemporary texts. The

11 A table illustrating the results can be found in Witte/Sâmihăian (2013, p. 15). 
general tendency is to have a balance between them or even to favour contemporary texts that are more accessible at this age.

For grade 12, all curricula emphasize the influence of the cultural and, with the exception of Finland, also of the linguistic model on the recommended criteria for choosing the texts to be studied. This means the progression towards a more sophisticated reader (as shown in Figure 1) is clearly marked.

We noticed that some curricula give more analytic or normative guidelines, thus implying an obligatory literary canon in upper secondary, by mentioning names of authors (Ro) or even titles of literary works $(\mathrm{Pt})$ to be studied. Others specify only the period or the type of literature (in terms of genres, cultural movements, concepts of literary theory etc.). Again, in the three German lands we can see more eclectic criteria for text selection. They can be associated with all the four models of studying literature. This indicates that the formal curricula of the German states are based on a rather open, non-normative attitude towards literary texts. For example, literature of the Middle Ages is included in Bavaria's curriculum, together with literature on adventure, and literature on adolescence yesterday and today, here and elsewhere.

Analysing the information regarding the presence of canon in the eight European curricula, we can see that an international canon is present both in lower and upper secondary (with two exceptions in grade 12, in the curricula of Po and $\mathrm{Nl}$ ), but the national canon seems to be more influential in the upper secondary. It is possible that in some cases, the canon is powerful enough that there is no need to mention it in the curriculum: it will still be influential. This seems to be the case of the German curricula, where there is no mentioning of the national canon in upper secondary, although experts from the project team and the group discussions told us that the national canon is not neglected at this stage, but present via central examinations.

These results can be considered representative for European tendencies in teaching literature and they validate our perspective regarding the line of cumulative paradigms presented in Figure 1. It seems that the canonic texts or authors are generally placed towards the end of secondary education, mainly in the last two grades. In the lower grades there is a tendency to focus more on the accessibility of a book (on students' background, interests and reading abilities), choosing popular genres for adolescents in order to help them discover the pleasures of reading. Towards the end of secondary school, students are supposed to read some representative, canonical literary works from national literature and from universal literature, too. This means the progression from a naive reader towards a more sophisticated reader is clearly followed. 


\subsubsection{The third dimension of the comparison: didactics}

The third dimension concerns didactics, or more explicitly strategies for teaching, learning and assessing students in literature classes. Although the formal curricula are not prescriptive in this respect, they offer some suggestions. For comparing didactics, we identified references to approach, class management, teacher role, and assessment in the analysed curricula (criteria 3, 5, 6, 7 in Table 1).

We have to mention that not all the analysed curricula refer to this dimension. This means that teachers have large space for choosing in this matter and that their responsibility for the decisions is high. We based our observations on the reports of the experts from the project team that provided us with some insights in this respect. In their reports, the experts of each of the six countries referred to: the didactic approaches, the type of learning activities used in the classroom, and the assessment methods.

From the reports and the data collected we could conclude that in lower secondary the dominant paradigm in the field of didactics is personal growth (six curricula), followed by the linguistic model (dominant in four curricula). In upper secondary, the cultural and the linguistic models are equally dominant (five curricula). But it is important to note that the differences are not so big between the score each model has got. ${ }^{12}$ This means that, in fact, even when dealing with a cultural or linguistic approach, didactic strategies should be varied and able to capture the interest of nowadays' students. The findings regarding this dimension also confirm our cumulative model of the literary teaching paradigms and are consistent with the results presented for the other two dimensions, student competences and books.

\subsubsection{Conclusions of the curriculum-comparison}

In spite of the difficulties of comparing and quantifying such fluid aspects as the presence of certain paradigms of studying literature in a curriculum, our analysis revealed a consistent image of the various ways of dealing with literature in lower and upper secondary in six European countries.

We tried to relate the findings of our research to the initial aim of the LiFT-2 project, which was to develop a European Framework for teachers of literature in secondary education. From this point of view, we were interested especially in identifying the lines of development of the literary competence in the analysed curricula and also in comparing them with our hypothesis that such a line should

12 See the table in Witte/Sâmihăian (2013, p. 17). 
start with a focus on the personal developmental model and then gradually cumulate with the social and the linguistic paradigm, for bringing forward the cultural model at the end of upper secondary (Figure 1). Within this line, students are guided in school to evolve from dependent, naïve and sometimes unmotivated readers of rather simple books to enthusiastic, autonomous and sophisticated readers of demanding literary works.

We can conclude that most of the analysed literary curricula for lower secondary (grade 7), are dominated by the personal and linguistic paradigms, while in upper secondary (grade 12) the cultural and linguistic paradigms prevail. Five of the eight curricula investigated turned out to mirror this developmental line, with the Dutch and Finnish curricula as the clearest representatives. On the other hand, the formal curricula of the Czech Republic, Portugal and Bavaria (Germany) diverged the most from this developmental line because they devote special attention to cultural literacy not just in grade 12, but in grade 7 also. These countries encourage development towards cultural reading from the start. It is possible that these differences reflect an influence of the Roman tradition, which even now is seen as the cultural watershed between Northern and Southern Europe (cf. Hofstede 2001). There are cultural traditions in developing each literary curriculum that become transparent in a deeper analysis. However, even if the number of curricula may not be representative for Europe, and we cannot draw any strong conclusion, it seems that a developmental line that defines the levels of literary competence is needed. Such a point of reference can help teachers to identify the level of the literary competence of students and also give them some didactic tools to realize the desired progress of students with different levels of literary competence. It can also help policymakers and curriculum designers to think critically about the literature curriculum in their country. As curriculum design is particularly restrictecd to national contexts and often stable traditions a 'fresh eye' could be helpful. We would thus argue that LiFT-2 could inform curriculum designers for secondary education, provide them with new perspectives and encourage developments towards a more student oriented approach.

Another important conclusion is that all national curricula are open to the reading of international literature. This means that the European reading list we included in the LiFT-2 framework can be used by most member states and thus can contribute to the formation of a European cultural identity.

Our findings resulted from the comparative analysis of literature curricula in some European countries. They were integrated by the project team in describing the levels of the literary competence, in suggesting types of books or titles for each level and in drawing up different transitions from one level to another, on 
the basis of didactic strategies and learning activities. Furthermore, they ensured the common basis for the development of the Framework.

\subsection{Procedures in LiFT-2}

At the heart of the design of the Framework were the expert-discussions. They form the centre of the procedures used by the project team (see figure 3) and were aiming at collecting as rich an insight into teachers perceptions and opinions as possible (cf. Witte et al. 2012, p. 11). Every partner-team carried out two expert-discussions with five to six teachers in light of designing the levels and two for designing the transitions. They chose the teachers themselves. Prior to the discussions the teachers rated a pile of well-known narrative texts as to grades in secondary education (named A to D). For one of the texts they did a so called quick scan: Following the guideline of the Quick Scan (now: Book Scan) ${ }^{13}$ they described the text with regard to the following dimensions: 1) general demands for engaging in the book, 2) familiarity with literary style, 3) familiarity with literary procedures, 4) familiarity with literary characters. Every discussion covered several rounds: First, teachers would describe learner characteristics of students of a certain grade and an average performance in literary reading. Then they would discuss what characteristics of the books they had considered as suitable for the respective grade would fit these learners and what characteristics wouldn't. Teachers were encouraged to discuss these features so that in the end the discussions would provide rich data to design the levels of literary development. Data were brought back into the international project team and discussed in a comparative perspective. The aim was to identify the common features of learners which the teachers had spelt out and achieve agreement on the number and character of levels.

13 The Quick Scan has been revised in the course of the project in close exchange with the experts. See http://de.literaryframework.eu/static/documents/de/Buch-Scan_LiFT2_2014_02_05.pdf. (22.01.2016). 
Fig. 3: Procedures in LiFT-2

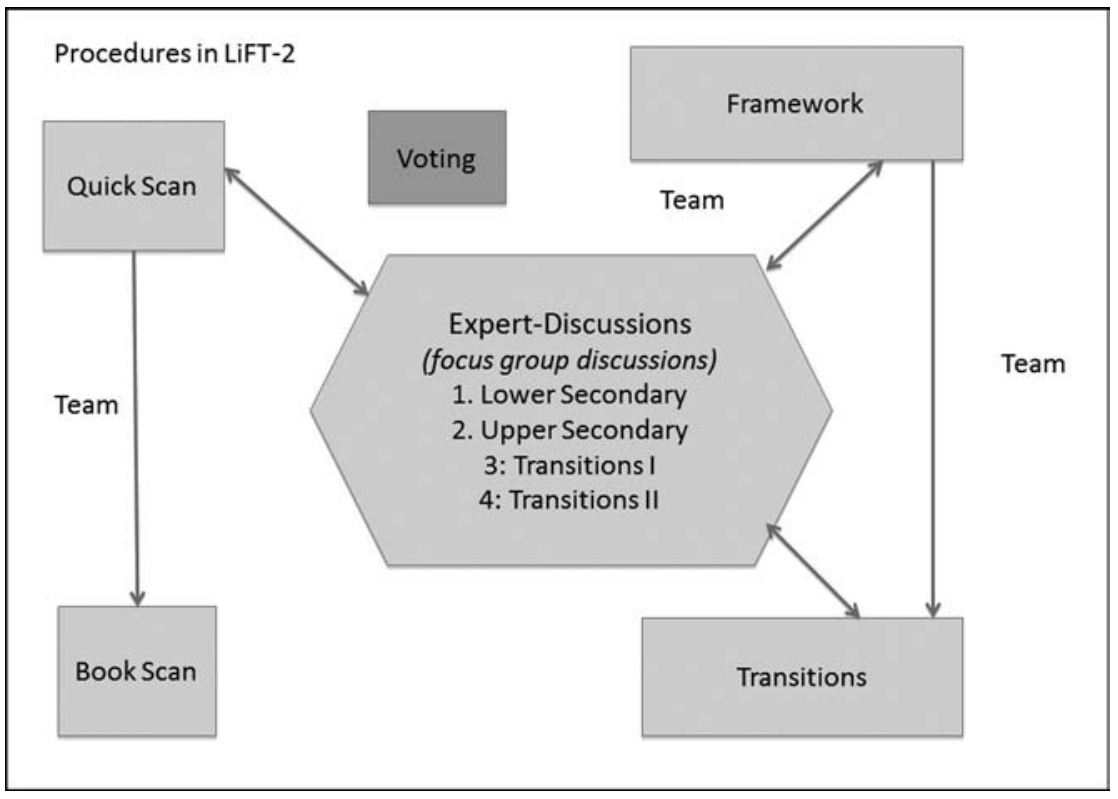

The synthesis done by the project team was brought back to the experts and validated. At the end of this process, a framework of four levels for age 12 to 15 and six levels for age 15 to 18 was confirmed. The different levels were named with a potentially expressive term and a short version of the Framework which covers just one sheet of paper was agreed upon. The levels are: Level 1 - Experiencing, Level 2 - Engaging, Level 3 - Exploring, Level 4 - Interpreting, Level 5 - Contextualizing, Level 6 - (pre-)Academic. ${ }^{14}$

In a further step transitions were designed in order to assist teachers to design lessons and sequences in literature education in light of progression. ${ }^{15}$ Again, expert-discussions were carried out, commonalities were identified in the international project team and the results were validated by the experts. Besides, the international team extended the Quick Scan in a didactical perspective, took into account the teachers' feedback and drafted the Book Scan.

14 The Framework can be accessed (in the various languages) via the website. An overview of the Framework in German is available here: http://de.literaryframework.eu/static/ documents/de/Framework_LiFT-2_deutsch_Feb2014_final.pdf. (22.01.2016).

15 On the website these transitions are spelt out under the headline 'how to lift'. 
In order to provide reliable book recommendations for the various levels an online validation procedure was set up in each participating country: Numerous teachers were asked to allocate books to the levels of the Framework by linking features of the book to the characteristics crucial to the levels. After setting up the website the books were integrated according to this voting procedure.

As can be seen on the website, there is a European page (symbolised by Johann Amos Comenius) and there are pages for each partner. The European page offers an international reading list. On the partner-pages numerous translations are included. Depending on local circumstances, current work with LiFT-2 - funded only till 2012 - varies. In Romania, the instruments are used in teacher education and teacher training. In Germany, new book recommendations are added frequently, book scans are offered that concern particular texts and student teachers are encouraged to assist in choosing suitable texts and offer book scans (particularly as part of their course work in university seminars).

\section{Conclusion: An International Developmental Project}

LiFT-2 could clearly work on common basis. The international team succeeded in drafting the Framework and this proves fruitful for both teachers and studentteachers in the various contexts. With regard to challenges and potentials of international research in $\mathrm{L} 1$ some traces of tensions should be acknowledged. These concern the dimensions of text, learners and procedures.

As to the texts international differences became obvious with regard to genre: While in most places, longer narratives, mainly novels form common features in the literature classroom, in Romania short stories and novelas are dominant, at least in the lower secondary. This affected the work with teachers as experts.

Expectations to what is 'good' literature or literature suitable for teaching may vary. For the project team, the novel Disgrace by South African writer J. Coetzee had some meaning but according to the Czech partners would be impossible in Czech literature education.

World literature is present to a different extent: In the Netherlands, only Dutch literature forms part of the list for upper secondary portfolio work. This leads to the exclusion of the genre fantasy and also of translations. The Finish partner pointed to a growing acceptance of world literature including literatures from Africa and South America in educational contexts. Translations are a quite accepted feature in literature for young readers in Germany, but not much present with other literature in German literature education. In Czech Republic literacy has been strongly stressed which affects the status of literature in education. 
Our attempts to include world literature and recent books in our book recommendations proved almost impossible via the book voting procedures because only well-known books got enough votes. We failed in encouraging teachers to read books they did not yet know in order to be able to vote which may well hint at the limited time frame teachers can offer for such procedures.

Besides, the voting demonstrated once more, how stable teaching traditions are: Despite the criteria to describe texts in the light of learners' abilities and motivations some historical texts were allocated to the traditional school grades without further considerations (e. g. the German $19^{\text {th }}$ century novelas which prove difficult for learners on the linguistic level, but also with regard to the narrative structures).

From the curriculum comparison we learn that on the level of curriculum there is more openness to progression and change with regard to text choice than is actually made use of.

With regard to the learners, one issue bothered us a lot: The relationship between school grades and age and school grades and forms of schooling is different between the various countries. Germany has the specific problem of having an extremely differentiated system of secondary education. This limits the possibilities to relate levels, grades and age. Thus, it has to be taken very seriously, that the levels are not clearly connected to a notion of age or "school age". This is why the framework for age 15 to 18 again starts with level 1 and thus hints at the fact that it is well possible to have learners at age 15 who could still be described by the features of level 1.

With regard to the procedures of the project we sometimes felt the restrictions of the limited funding in the EU-Comenius-program. The project partners would have liked to have the expert-discussions all taped, transcribed and translated into English but this was impossible because of these limits. This restriction of course limits the research options with the data collected. In a more general perspective, this shows that international research needs to acknowledge the higher demand of resources for ensuring common work on data from different linguistic contexts.

We also experienced the hardship of ensuring a common language among the project partners. English was our working language, for none of us English was the mother tongue. Thus, the wording of the Framework was particularly difficult. Addressing native speakers and translators for help sometimes even enriched our discussion: We would then have even more versions, but would still have to work out which would fit best.

Ensuring the outcome on the level of publications also proved difficult: To run an international website and a forum together has limits. In fact, it proved 
necessary to gain more independence with partners in the long run - again an economical issue.

It has to be acknowledged that our expert-groups were crucial. In the frame of qualitative research small samples are acceptable. However, given the international diversity the project might have benefited from more groups per country. Still, the validation process was smooth and coherence between partners encouragingly strong.

As to the potentials of our project we can also raise a few points: The curriculum comparison shows that the different partners all have poly-paradigmatic curricula. It is to be expected that at least within Europe commonalities on the level of curriculum are strong enough to research the field of L1 education in an international perspective - under the presupposition of an awareness of potential differences.

LiFT-2 is a pragmatic project in many respects: The project plan could rely on a model, the sample was small, and we have pointed to some of the limits of our work. However, the project apparently succeeds in providing a sensible tool for different contexts, both for in-service and pre-service teacher training. In reaching out to practitioners the project seems particularly rich in its clear ambition to remain close to teaching, to the classroom experience of teachers and their pedagogical content knowledge, also to the language which is used by professionals and in literature teaching at school (rather than at university). Encounters with the teachers as experts in the project were encouraging and illuminating for both sides. Teachers generally appreciated the expert-discussions as a forum to share and develop their knowledge and professional praxis.

Hence, LiFT-2 shows that international research and development can benefit from national research and development. It also offered a platform to share differences and learn about teaching traditions and the research approaches the respective partners would follow. Its result, namely the Framework, reaches out to an international audience and can ease academic discussion, also because it offers instruments in a potentially common language, that is 'working English'. Thus, we would argue that LiFT-2 contributes to international understanding in literature education and at the same time to an awareness of difference. It even may help to stabilise and develop a common style of thinking among protagonists of research and development in L1 literature education.

With regard to new research questions of common interest in an international context an extension of the Framework would be fruitful. This extension could concern younger learners (grade 3-6) and other genres, particularly poetry. Besides, it would be beneficial to explore the traces of tensions further: What are the differences on the level of textbooks, teaching material and tasks? What are 
the differences on the level of teaching, including the value and relevance of literature in L1 education? What are the conditions of successful implementation of the Framework in teaching? Finally, the various aspects of difference could be explored in their interrelatedness: The national contexts bring about various forms of schooling, teacher-training differs etc. Thus, national differences, though they did not serve as a starting point in the LiFT-2 project (as in IMEN), became accessible to some extent and lead to new research questions which could be dealt with on an international level.

\section{References}

Aase, Laila/Fleming, Mike/Pieper, Irene/Sâmihăian, Florentina (2007): Text, literature and „Bildung“ - comparative perspectives. In: Pieper, Irene (Ed.)/Aase, Laila/Fleming, Mike/Sâmihăian, Florentina: Text, literature and "Bildung". Language Policy Division, Council of Europe, Strasbourg, Retrieved from: https://www.coe.int/t/dg4/linguistic/prague_studies07_EN.asp, pp. 7-30. (22.01.2016).

Council of Europe/Language Policy Unit (2006-2016): Languages in Education, Languages for Education. A platform of resources and references for plurilingual and intercultural education. Ad-hoc coordination group: Laila Aase, Jean-Claude Beacco, Michael Byram, Marisa Cavalli, Daniel Coste, Alexandru Crisan, Michael Fleming, Olivier Maradan, Sigmund Ongstad, Irene Pieper, Florentina Sâmihăian, Helmut Vollmer and Piet-Hein van de Ven. Editorial board: Jean-Claude Beacco, Michael Byram, Daniel Coste, Michael Fleming (various studies and still ongoing). http://www.coe.int/t/dg4/linguistic/langeduc/le_platformintro_EN.asp. (22.01.2016).

Doecke, Brenton/Van de Ven, Piet Hein (Eds.) (2011): Literary Praxis. A Conversational Inquiry into the Teaching of Literature. Rotterdam: Sense.

European Commission (2006). The European Framework for Key Competences for Lifelong Learning. In: Annex of a Recommendation of the European Parliament and of the Council of 18 December 2006 on key competences for lifelong learning (2006/962/EC). Official Journal of the European Union, 30.12.2006. Retrieved from: http://eur-lex.europa.eu/homepage.html. (22.01.2016).

Fleck, Ludwik (2012) [1935]: Entstehung und Entwicklung einer wissenschaftlichen Tatsache - Einführung in die Lehre vom Denkstil und Denkkollektiv. 10. Aufl. Frankfurt a. M.: Suhrkamp.

Fleming, Michael (2010): The Literary Canon: Reflections from a School Curriculum Perspective. In: Sâmihăian, Florentina (Ed.): The literary canon approaches to teaching literature in different contexts. Bucharest: University of Bucharest Publishing House, pp. 11-20. 
Herrlitz, Wolfgang/Ongstad, Sigmund/van de Ven, Piet-Hein (ed.s) (2007): Research on Mother Tongue Education in a Comparative International Perspective. Theoretical and Methodological Issues. Amsterdam: Rudopi.

Hofstede, Geert (2001): Culture's Consequences: Comparing Values, Behaviors, Institutions, and Organizations across Nations. $2^{\text {nd }}$. Ed. Thousand Oakes (CA): Sage.

Ongstad, Sigmund/Van de Ven, Piet-Hein/Buchberger, Irina (2004): Mother tongue didaktik (sic). An International Study Book. Schriften der Pädagogischen Akademie des Bundes in Oberösterreich 21. Linz: Trauner.

Pieper, Irene (2014): Den Schüler vor Augen, den Anspruch im Sinn: Der internationale Referenzrahmen LiFT-2 zu Progression und Textauswahl für den Literaturunterricht der Sekundarstufen. In: Frederking, Volker/Krommer, Axel/Meier, Christel (Hrsg.): Taschenbuch des Deutschunterrichts. Bd. 3. Baltmannsweiler: Schneider, S. 586-609.

Pieper, Irene (2006): The Teaching of Literature. Strasbourg: Language Policy Division, Retrieved from: www.coe.int/t/dg4/linguistic/Source/Pieper_EN.doc. (22.01.2016).

Prensky, Marc (2010): Teaching Digital Natives: Partnering for Real Learning. Thousand Oaks: Corwin.

Rijlaarsdam, Gert/Janssen, Tanja (1996): How do we evaluate the literature curriculum? About a social frame of reference. In: Marum, Ed (Ed.): Children and books in the modern world. Contemporary perspectives on literacy. London/ Washington DC: The Falmer Press, pp. 75-98.

Roberg, Thomas/Susteck, Sebastian/Müller-Michaels, Harro (Hrsg.) (2010): Geschichte des Deutschunterrichts von 1945-1989 (Teil 2): Deutschunterricht im Widerstreit der Systeme. Frankfurt a. M.: Peter Lang.

Sawyer, Wayne/Van de Ven, Piet-Hein (2007): Starting Points: Paradigms in mother-tongue education. In: L1 - Educational Studies in Language and Literature. Vol. 7. No. 1, pp. 5-20.

Shulman, Lee S. (1986): Those Who Understand: Knowledge Growth in Teaching. In: Educational Researcher. Vol. 15. No. 2, pp. 4-14.

Veen, Wim/Vrakking, Ben (2007): Homo Zappiens: Growing up in a digital age. Network Continuum.

Verboord, Marc (2005): Long-term effects of literary education on book-reading frequency: An analyses of Dutch student cohorts 1975-1998. In: Poetics. Vol. 33. No. 5-6, pp. 320-342.

Wiemer, Thomas (2011): Ideen messen, Lektüren verwalten? Über Qualitätskriterien literaturwissenschaftlicher Forschung. In: Journal of Literary Theory. Vol. 5. No. 2, pp. 263-278. 
Witte, Theo (2008): Het oog van de meester: een onderzoek naar de literaire ontwikkeling van havo-en vwo-leerlingen in de tweede fase van het voortgezet onderwijs. Delft: Eburon.

Witte, Theo/Janssen, Tanja/Rijlaarsdam, Gert (2006): Literary competence in L1 and the literature curriculum. Paper presented on the International Colloquium Mother Tongue Education in a Multicultural World: Case Studies and Networking for Change (Sinaia - Romania, June 22 to 25, 2006).

Witte, Theo/Rijlaarsdam, Gert/Schram, Dick (2012): An empirically grounded theory of literary development. Teachers' pedagogical content knowledge on literary development in upper secondary education. In: L1 Educational Studies in Language and Literature. Vol. 12 (Special issue guest edited by Irene Pieper \& Tanja Janssen), pp. 1-33.

Witte, Theo/Sâmihăian, Florentina (2013): Is Europe open to a student-oriented framework for literature? A comparative analysis of the formal literature curriculum in six European countries. In: L1-Educational Studies in Language and Literature. Vol. 13, pp. 1-22.

Project links:

http://www.literaryframework.eu

http://de.literaryframework.eu/keydocuments.html

http://de.literaryframework.eu/static/documents/de/Buch-Scan_LiFT2_2014_02_05.pdf 
Iris Winkler, Matthias Heinrich, Astrid Fischer, Ulrike Krause

\title{
Multiperspektivität in der Lehrerbildung. Fächerübergreifendes Lernen in der Mathematik- und Deutschdidaktik
}

\begin{abstract}
In the present project we investigate how multiple perspectives in teacher education foster professional competencies of pre-service teachers of German and Mathematics. Multiple perspectives will be implemented by interdisciplinary learning that combines perspectives of Mathematics and German pedagogical content knowledge.
\end{abstract}

Deutsch und Mathematik sind zentrale Fächer im schulischen Fächerkanon. Zugleich werden sie in der Regel als sehr unterschiedliche Schulfächer erlebt (z. B. Haag/Götz 2012). Dass ein Reiz darin liegt, vermeintlich Gegensätzliches aufeinander zu beziehen, zeigen bereits frühere Arbeiten, die Möglichkeiten fächerübergreifenden Unterrichtens in Mathematik und Deutsch für die Schule ausloten (Gallin et al. 1985; Knapp 2001; Knapp/Pfaff 2008). In dem Projekt, das in diesem Beitrag vorgestellt wird, untersuchen wir, inwieweit fächerübergreifendes Lernen in der Mathematik- und Deutschdidaktik zur Förderung fachdidaktischer Kompetenzen bei Lehramtsstudierenden beider Fächer beiträgt. Es geht also darum, interdisziplinäre Lernumgebungen in den beiden Fächern zu entwickeln und deren Wirksamkeit in Interventionsstudien zu überprüfen.

Als beteiligte Disziplinen kooperieren Mathematik- und Deutschdidaktik sowie die pädagogische Psychologie. Das Projekt ist im doppelten Sinn multiperspektivisch angelegt: Auf der Ebene der Forschungskooperation ermöglicht erst die Kombination der unterschiedlichen Fachperspektiven die Ausschärfung einer fächerübergreifenden Fragestellung sowie des methodischen Vorgehens (s. u., Abschnitt 1 und 2). Auf der Ebene der geplanten Intervention sollen die Studierenden eine andere Perspektive auf ihr eigenes Fach einnehmen, indem sie es aus der Perspektive des jeweils anderen Faches betrachten. Neben der Ausdifferenzierung fachdidaktischen Wissens erwarten wir dadurch eine Reflexion fachdidaktisch relevanter Überzeugungen, die sich auf das eigene Fach beziehen (s. u., Abschnitt 2 und 3). 


\section{Fundierung fachdidaktischer Forschungsfragen in einer fächerübergreifenden Perspektive}

Unter fachdidaktischer Kompetenz verstehen wir die Disposition, fachdidaktische Problemstellungen fundiert und situationsadäquat zu lösen. Fachdidaktisches Wissen bildet diesem Verständnis zufolge - wie in den Modellen professioneller Lehrerkompetenzen von TEDS-LT (Teacher Education and Development Study - Learning to Teach) und verwandten Studien (Blömeke 2011) sowie von COACTIV (Professionswissen von Lehrkräften, kognitiv aktivierender Mathematikunterricht und die Entwicklung mathematischer Kompetenz; Baumert/ Kunter 2011b) - einen Teilbereich professionellen Lehrerwissens. Es ist davon auszugehen, dass professionelles Wissen, also kognitive Kompetenzfacetten, und emotionale, motivationale sowie einstellungsbezogene Kompetenzfacetten auch bei der Lösung fachdidaktischer Problemstellungen interagieren (z. B. Baumert/ Kunter 2011b; Blömeke 2011; Weinert 2001).

Die Annahme, dass sich fachdidaktische Kompetenzen von Lehramtsstudierenden durch fächerübergreifende Lernumgebungen fördern lassen, macht die Klärung zweier grundlegender Fragen in der ersten Phase des Forschungsprozesses erforderlich. Zum einen müssen Ziel- bzw. Problemstellungen in der Lehre identifiziert werden, die in beiden beteiligten Fachdidaktiken relevant sind. Diese strukturellen Gemeinsamkeiten bilden die Grundvoraussetzung, um überhaupt eine fächerübergreifende Forschungsfrage zu formulieren. Zum anderen gilt es Unterschiede zwischen den Fächern zu beschreiben, die aus Sicht der Forschenden oder aus Sicht der Studierenden bestehen. Denn diese Unterschiede sind es, die in den fächerübergreifenden Lernumgebungen als Reflexionsimpulse genutzt werden sollen.

Eine Problemstellung, die eine wichtige Rolle im Diskurs der Mathematik- wie der Deutschdidaktik spielt, ist die Frage nach dem fachdidaktischen Potenzial von Aufgabenstellungen. Lern- und Leistungsaufgaben im Hinblick auf ihr Anforderungsprofil einzuschätzen, ist eine zentrale fachdidaktische Aufgabe im Unterrichtsalltag (vgl. z. B. Büchter/Leuders 2014; Köster, im Druck; Maier et al. 2014; Winkler 2010). In unserem Projekt fungiert die adäquate fachdidaktische Einschätzung von Aufgabenstellungen als Zieldimension fachdidaktischer Lehre als zentrales Element, das die Fächer Deutsch und Mathematik verbindet. Die situationsgerechte Bewältigung dieser Anforderung setzt fachdidaktische Kompetenz voraus. Dabei sind nicht nur kognitive Kompetenzfacetten gefordert, sondern es kommen auch einstellungsbezogene Facetten professioneller Kompetenz ins Spiel.

Für die Modellierung kognitiver Kompetenzfacetten bei der Aufgabeneinschätzung ist im Projekt eine fächerübergreifende Lösung erforderlich. Dabei kann an ausführliche Vorarbeiten insbesondere aus der Mathematikdidaktik angeknüpft 
werden. Dies ist aus Sicht der Deutschdidaktik ein Beispiel dafür, wie zielführend der Blick über den Tellerrand der eigenen Disziplin sein kann. Die Mathematikdidaktik unterscheidet in der Studie TEDS-M (Teacher Education and Development Study in Mathematics; Blömeke et al. 2010) folgende Wissensbereiche, die mit Blick auf das Aufgabenstellen zentral sind: Wissen über aufgabenrelevantes Schülervorwissen, Wissen über schwierigkeitsbestimmende Aufgabenmerkmale, Wissen über verbreitete fachspezifische Schülervorstellungen und Wissen über angemessenes Feedback (Döhrmann et al. 2010; Laschke/Döhrmann 2014; Laschke/ Kaiser 2014). TEDS-M und weiterführend auch TEDS-LT (an TEDS-LT ist auch die Deutschdidaktik beteiligt) differenzieren in Anknüpfung an vorangegangene Studien fachdidaktisches Wissen nicht nur nach inhaltlichen Aspekten, sondern auch nach kognitiven Prozessen beim Einsatz des Wissens. Bewährt hat sich diesbezüglich die Unterscheidung der Operationen Kennen/Erinnern/Abrufen, Anwenden, Bewerten/Begründen (Bremerich-Vos/Dämmer 2013; BremerichVos et al. 2011; Buchholtz/Kaiser 2013; Buchholtz et al. 2011; Döhrmann et al. 2010). In unserem Forschungsvorhaben legen wir für die fachdidaktische (Teil-) Kompetenz im Bereich der Auswahl, Präsentation und Auswertung von Aufgabenstellungen entsprechend folgendes Modell kognitiver fachdidaktischer Kompetenzfacetten zugrunde (Abb. 1):

Abb. 1: Fachdidaktische Kompetenzfacetten im Bereich der Aufgabeneinschätzung: Wissensdimensionen und kognitive Operationen

\begin{tabular}{|c|c|c|c|}
\hline $\begin{array}{ll}\text { Wissensdimension } & \begin{array}{l}\text { Kognitive } \\
\text { Operationen }\end{array} \\
\end{array}$ & $\begin{array}{l}\text { Erinnern, } \\
\text { Abrufen, } \\
\text { Benennen }\end{array}$ & $\begin{array}{l}\text { Anwenden, } \\
\text { Herstellen von } \\
\text { Zusammenhängen }\end{array}$ & $\begin{array}{l}\text { Reflektieren, } \\
\text { begründetes } \\
\text { Beurteilen }\end{array}$ \\
\hline $\begin{array}{l}\text { Wissen über } \\
\text { schwierigkeitsbestimmende } \\
\text { Aufgabenmerkmale }\end{array}$ & & & \\
\hline $\begin{array}{l}\text { Wissen über aufgabenrelevantes } \\
\text { Schülervorwissen }\end{array}$ & & & \\
\hline $\begin{array}{l}\text { Wissen über aufgabenspezifische } \\
\text { Schülervorstellungen }\end{array}$ & & & \\
\hline $\begin{array}{l}\text { Wissen über } \\
\text { lernunterstützende Rückmeldungen }\end{array}$ & & & \\
\hline
\end{tabular}

Wir gehen davon aus, dass (angehende) Lehrpersonen beim Einsatz von Aufgabenstellungen Wissen in allen vier Wissensbereichen benötigen und dass sie prinzipiell in der Lage sein müssen, je nach Anforderungssituation mit diesem Wissen alle drei 
Typen kognitiver Operationen durchzuführen. ${ }^{1}$ Der nächste Schritt im Forschungsprojekt besteht darin, ein Testinstrument zu entwickeln, mit dem sich das aufgabenbezogene Wissen von Studierenden ebenso erfassen lässt wie die kognitiven Operationen, mit deren Hilfe es genutzt wird. Für Studierende der Fächer Deutsch und Mathematik sind zwar fachspezifische Tests erforderlich. Diese müssen im vorliegenden Projekt aber eine fächerübergreifende Auswertung ermöglichen, damit sich die Ergebnisse sinnvoll vergleichen lassen. Für die Tests haben wir entsprechend ein zwar fachspezifisches, aber parallel angelegtes offenes Aufgabenformat entwickelt. Zum Aufgabenstamm gehören jeweils exemplarische Schüleraufgaben und darauf bezogene Schülerantworten, die durch die Studierenden zu analysieren sind. Für die Bearbeitung der Aufgaben sind die Wissensdimensionen und die kognitiven Operationen des hier skizzierten Modells erforderlich. Erste Pilotierungsergebnisse bestätigen unsere Annahme, dass das Modell eine gute Grundlage für die fächerübergreifende Auswertung von Studierendenlösungen bietet.

Auch was die einstellungsbezogenen Kompetenzfacetten betrifft, die beim Aufgabeneinsatz im Fachunterricht wirksam werden, geht es zunächst darum, Parallelen und Unterschiede zwischen beiden Fächern festzustellen. Beobachtungen in der Lehre legen nahe, dass sowohl viele Mathematik- als auch viele Deutschstudierende simplifizierende Vorstellungen vom jeweils eigenen Fach haben. Für das Fach Mathematik liegen etliche Untersuchungen zu fachspezifischen epistemologischen Überzeugungen von Lernenden wie (angehenden und praktizierenden) Lehrpersonen vor (Köller et al. 2000; Schmotz et al. 2010; Schoenfeld 1992; Voss et al. 2011; Schmeisser et al. 2013). Als problematisch für den Kompetenzerwerb im Mathematikunterricht erweist sich dabei die Vorstellung von Mathematik als statischer, schematisch-algorithmisch ausgerichteter Disziplin (Köller et al. 2000) und von Mathematikunterricht als rein transmissivem Prozess, der sich auf das Vermitteln von Algorithmen beschränkt. Teilen Lehrkräfte diese Vorstellung, hat dies nachteilige Auswirkungen auf den Lernerfolg ihrer Schülerinnen und Schüler (Voss et al. 2011). Bei Studierenden zu Beginn des Lehramtsstudiums der Mathematik sind transmissive Vorstellungen von Mathematikunterricht stärker ausgeprägt als bei erfahrenen Mathematik-Lehrkräften (Schmeisser et al. 2013).

1 Dass zwischen den kognitiven Operationen Abhängigkeiten bestehen, ist nichts Neues: Wer sich an benötigtes Wissen nicht erinnert, kann es nicht in Zusammenhänge stellen geschweige denn reflektieren. Wer keine Zusammenhänge herstellen kann, wird sich mit dem Reflektieren schwertun. Allerdings wäre es ein Trugschluss anzunehmen, dass Erinnern und Benennen immer einfach wäre (man denke nur an komplexe fachliche Inhalte) und Reflektieren stets am schwierigsten. 
Für das Fach Deutsch fehlen systematische empirische Untersuchungen zu fachspezifischen Überzeugungen. Was Überzeugungen zum Lehren und Lernen im Literaturunterricht betrifft, liegen allerdings einhellige Hinweise vor. Um der domänenspezifischen Komplexität und Ambiguität bei der Arbeit mit literarischen Texten im Unterricht zu begegnen, erachten Deutschlehrkräfte anscheinend vorwiegend drei Bewältigungsvarianten für angemessen. Die ersten beiden Varianten können als ein Ausdruck transmissiver Vorstellungen gelten, die letzte als ein Ausdruck des Gegenteils: schematisches Abarbeiten von Algorithmen der Literaturanalyse (z. B. Kämper-van den Boogaart 2003; Zabka 2012), die Vorgabe von Deutungen (z. B. Vogt 2004; Willenberg 2002) oder weitestgehende Öffnung des Unterrichts für individuell beliebige Zugangsweisen und Interpretationen (Härle 2004; Vogt 2004). Alle drei Varianten sind als Formen der "oversimplification“ (Spiro et al. 1992) zu betrachten und aus einer fachlichen und fachdidaktischen Perspektive in ihrer Einseitigkeit wenig geeignet für die Förderung des Kompetenzerwerbs der Lernenden im Bereich der Literaturanalyse.

Bislang fehlen Untersuchungen zur Frage, wie (angehende) Mathematik- und Deutschlehrkräfte die „Philosophie“ ihres jeweiligen Schulfaches (Bromme 1992) im Vergleich zum anderen Fach sehen. Dass die Charakteristika beider Fächer von Schülerinnen und Schülern als massiv gegensätzlich erlebt werden, zeigt eine Studie von Haag/Götz (2012). Aus einer eigenen Pilotstudie liegen erste Befunde vor, wie Lehramtsstudierende der Fächer Deutsch $(\mathrm{N}=82)$ und Mathematik $(\mathrm{N}=79)$ die Besonderheiten des jeweils eigenen Faches im Vergleich zum jeweils anderen sehen (Winkler 2015a; siehe auch unten, Abschnitt 3).

Den Deutschstudierenden wurde folgende Frage gestellt: „Deutsch und Mathematik werden als sehr unterschiedliche Schulfächer erlebt. Was ist aus Ihrer Sicht das Besondere an Deutsch gegenüber Mathematik?" Auf der anderen Seite wurden die Mathematikstudierenden befragt, was aus ihrer Sicht das Besondere an Mathematik gegenüber Deutsch ist. Das offene Antwortformat ermöglicht den Befragten, eigene Schwerpunkte zu setzen und das ihnen subjektiv Wichtige zu betonen. Durch den Auftrag, die Fächer zu vergleichen, wird das eigene Fach klar profiliert. Darüber hinaus erhält man so Aussagen zu beiden Schulfächern aus der Perspektive zweier Studierendengruppen, die diesen Fächern unterschiedlich nahestehen. Dass die durch die Frage geforderte Kontrastierung der beiden Fächer auch eine gewisse Verengung der Perspektive auf ganz bestimmte Fachmerkmale mit sich bringt, muss bei der Interpretation der Befunde reflektiert werden (wenn mit den Studierendenaussagen in der Lehre gearbeitet werden soll, wie in Abschnitt 3 vorgeschlagen, spielt dieser Aspekt aber eine untergeordnete Rolle). 
Sowohl die Mathematik- als auch die Deutschstudierenden zeigen in Bezug auf das Problemlösen im eigenen und im jeweils anderen Fach simplifizierende Vorstellungen, die größtenteils genau gegenläufig sind: Im Mathematikunterricht ist alles objektiv und eindeutig, im Literaturunterricht ist alles subjektiv und offen, so die dominierende Auffassung der Studierenden. Diese zugeschriebenen Facheigenschaften werden von den Studierenden der beiden Fächer jeweils gegenläufig als positiv bzw. negativ beurteilt (das hohe Maß an Subjektivität im Literaturunterricht bspw. als positives Freiheitsmerkmal aus Sicht der Deutschstudierenden und als Ausdruck von Beliebigkeit und Willkür aus Sicht der Mathematikstudierenden).

Für eine große Zahl der Lehramtsstudierenden im Fach Deutsch besteht den Pilotbefunden zufolge die begründete Annahme, dass sie in Bezug auf ihr Fach auf einem „multiplist level“ (Kuhn 2009) verharren, also davon ausgehen, dass literarisches Verstehen im Unterricht beliebig auf individuelle Ansichten gestützt werden kann. Bei den Mathematikstudierenden erscheint dagegen ein „absolutist level“ problematisch (Kuhn 2009), auf dem die Mathematik als eine Ansammlung von statischem Wissen über Aussagen und Verfahren gesehen wird (statisch-transmissive Perspektive, s. o.). Beide Auffassungen sind als ungünstige lehrerseitige Voraussetzung einzuschätzen, wenn es um das Stellen kognitiv anregender Aufgaben bzw. um den sachangemessenen und lernförderlichen Umgang mit Schülerantworten im Unterricht geht.

Vor diesem Hintergrund kann mit Blick auf einstellungsbezogene Kompetenzfacetten als Zieldimension des Lehramtsstudiums im Bereich Fachdidaktik in beiden Fächern gelten, dass fachliche Aufgabenstellungen und ihre Bearbeitungen auf einem „evaluativist level“ (Kuhn 2009) betrachtet werden. Das heißt, dass bei der Einschätzung von Aufgabenanforderungen weder schematische noch beliebige Erwartungen anzulegen sind, sondern dass Problembearbeitungen nach sachbezogenen Kriterien geprüft und Bewertungen unter Einbeziehung multipler Perspektiven argumentativ gestützt werden sollen. In dieselbe Richtung zielt auch eine Schlussfolgerung, die aus den COACTIV-Befunden zum fachdidaktischen Wissen von Mathematiklehrkräften gezogen wird. Diesen Befunden zufolge ist bei (angehenden) Lehrkräften die fachdidaktische Teilkompetenz zu fördern, multiple Lösungswege für fachliche Aufgaben zu erkennen und zu beurteilen (Baumert/Kunter 2011a).

Sowohl kognitive als auch einstellungsbezogene Kompetenzfacetten, die bei der fachdidaktischen Einschätzung von Aufgabenanforderungen eine Rolle spielen, sollen in der geplanten Intervention durch multiperspektivische Lehr-Lern-Settings gefördert werden, indem in die fachdidaktische Lehre des einen Faches fachdidaktische Perspektiven aus dem jeweils anderen Fach integriert werden. 


\section{Multiple Perspektiven durch fächerübergreifendes Lernen}

Die Präsentation multipler Perspektiven (vgl. Cognition and Technology Group at Vanderbilt 1997; Reinmann/Mandl 2006) zielt darauf ab, Reflexionsprozesse und den Erwerb transferierbaren Wissens zu fördern. Hintergrund des vorliegenden Projekts ist der Cognitive-Flexibility-Ansatz (Spiro et al. 2003), bei dem zur Förderung des Kompetenzerwerbs in wenig strukturierten Domänen gezielt mit multiplen Perspektiven und Kontexten gearbeitet wird. Die Lerninhalte werden in mehreren verschiedenen Zusammenhängen betrachtet. Dies ermöglicht es den Lernenden, von Oberflächenmerkmalen zu abstrahieren, zugrundeliegende Strukturen zu erkennen und eine ganzheitlichere Sicht auf ein Problem zu erhalten. Auf diese Weise werden die neuen Informationen vielfältig vernetzt, es wird Fehlkonzepten und einseitigen Sichtweisen vorgebeugt und ein tieferes Verständnis gefördert (vgl. Krause 2007). Der Einsatz multipler Perspektiven und Kontexte, beispielsweise die Betrachtung einer bestimmten Lösungsprozedur in unterschiedlichen Zusammenhängen, sollte den Transfer auf eine neue Problemstellung erleichtern. Insbesondere dürften multiple Perspektiven und Kontexte kognitive Flexibilität bei der Nutzung der erworbenen Kenntnisse und Fertigkeiten fördern (vgl. Spiro et al. 2003). Verschiedene Studien weisen auf positive Effekte von Multiperspektivität hin, sofern instruktionale Unterstützung erfolgt (s. u.). Für die Wirksamkeit von Multiperspektivität sind verschiedene Lernvoraussetzungen relevant, u. a. die Ambiguitätstoleranz der Lernenden (Dalbert 1999; Hartinger et al. 2005).

Multiple Perspektiven und Kontexte wurden in zahlreichen Studien als fester Bestandteil der jeweiligen Lernumgebungen eingesetzt (z. B. Busse/Krause 2015; Krause/Stark 2010; Krause et al. 2009; Krause et al. 2011). Eine systematische Untersuchung der Lernwirksamkeit multipler Perspektiven und Kontexte erfolgte u. a. im Rahmen von Studien im Bereich Wirtschaftswissenschaften (z. B. Stark 2000; Stark et al. 1999). Generell weisen die Ergebnisse darauf hin, dass die Auseinandersetzung mit multiplen Perspektiven und Kontexten hinsichtlich des Erwerbs komplexer Kompetenzen sehr lernwirksam ist, wenn instruktionale Unterstützung erfolgt. Wird beim Lernen mit multiplen Perspektiven bzw. Kontexten jedoch keine geeignete Unterstützung gegeben, führt dies leicht zu Überforderung (vgl. Stark et al. 2001). Ein Indikator hierfür ist eine erhöhte kognitive Belastung (Sweller 2010) im Lernprozess.

Im vorliegenden Projekt werden multiple Perspektiven durch fächerübergreifendes Lernen realisiert. Zwar studieren angehende Sekundarstufenlehrkräfte in der Regel zwei Fächer, so dass sie im alltagstheoretischen Sinn durchaus einen Vergleich zweier unterschiedlicher Fachkulturen haben. Im Projekt fungiert der Vergleich aber als gezielte Methode, die zur Erkenntnisgewinnung eingesetzt 
wird, insbesondere zum besseren Verständnis der eigenen Fachkultur durch Durchbrechung der Selbstreferenzialität in der Auseinandersetzung mit dem jeweils anderen Fach (Esser 2012; Schriewer 2003; Tainio/Winkler 2014). Für die Kombination der Perspektiven der Deutsch- und der Mathematikdidaktik werden zwei Zugänge gewählt. Zum einen beschäftigen sich die Studierenden mit Schüleraufgaben und Schülerantworten aus dem jeweils anderen Fach und ziehen daraus Schlussfolgerungen für die Einschätzung von Aufgabenanforderungen und Schülerantworten im eigenen Fach: Gibt es Parallelen zwischen Mathematik- und Deutschaufgaben? Ist beim fachlichen Lernen im Mathematikunterricht tatsächlich alles eindeutig und im Literaturunterricht individuell beliebig? Inwieweit sind Lösungsstrategien und Lösungsanforderungen des anderen Faches auf das eigene Fach übertragbar (z. B. Vielfalt von Lösungswegen, Kriterien für korrekte bzw. falsche Lösungen, Kriterien für schlüssiges Argumentieren)? Zum anderen werden wahrgenommene Charakteristika des jeweils eigenen Faches problematisiert, indem fremde Perspektiven darauf reflektiert werden. Dazu werden Studierendenaussagen aus der Pilotstudie (s. o., Abschnitt 1) genutzt. Deutschstudierende setzen sich mit Merkmalen von Deutschunterricht aus Sicht von Mathematikstudierenden auseinander, und Mathematikstudierende befassen sich mit Aussagen von Deutschstudierenden zum Mathematikunterricht. Diesen zweiten Zugang der geplanten Intervention erläutert der folgende Abschnitt genauer.

\section{Reflexion fremder Perspektiven auf das eigene Fach}

\subsection{Vorüberlegungen}

Selbstverständlich ist es interessant, was Mathematik- und Deutschstudierende jeweils von ihrem eigenen Fach denken, weil dies wesentliche Anknüpfungspunkte für die fachdidaktische Lehre aufzeigt. Ausgangspunkt der Intervention sind hier unter dem Vorzeichen der Multiperspektivität aber fachfremde Vorstellungen vom eigenen Fach. Grundlegend gilt dabei: Wenn in der fachdidaktischen Lehre die Überzeugungen von Studierenden anderer Fächer über das eigene Fach thematisiert werden, darf es keinesfalls darum gehen, diese Überzeugungen als falsch abzuqualifizieren. Stattdessen sollte deutlich werden, dass dies Überzeugungen sind, die sich in der Regel bei Lernenden während der Schulzeit in Bezug auf das jeweilige Fach gebildet haben. Vor diesem Hintergrund sind in der fachdidaktischen Lehre folgende Fragen zu diskutieren:

- Welche Schwerpunkte zeigen sich in den fachbezogenen Überzeugungen der fachfremden Studierenden? Ziel ist zunächst also eine vorwiegend deskriptive bzw. rekonstruktive Erfassung der exemplarischen Studierendenaussagen. Dadurch 
erwerben die angehenden Lehrpersonen u. a. Wissen über potentielle Schülervorstellungen im studierten Fach. Dieses Wissen ist für die fachdidaktische Aufgabeneinschätzung allgemein bedeutsam (vgl. oben, Abb. 1).

- Welche Ursachen für die geäußerten Überzeugungen könnten im Fachunterricht selbst liegen? Indem mit den Augen von Personen, die als eher nicht fachaffin einzuschätzen sind, auf den Unterricht im eigenen Fach geschaut wird, treten möglicherweise Unterrichtsmerkmale in den Vordergrund, die aus fachimmanenter Perspektive tradiert und kaum infrage gestellt werden. Diesbezüglich zielt die Intervention also auf die Reflexion und Weiterentwicklung einstellungsbezogener Kompetenzfacetten ab.

- Inwieweit sollen Lernende ihre Überzeugungen in Bezug auf das jeweilige Fach differenzieren? Wie kann der Fachunterricht dazu beitragen? Dieser Aspekt greift normative Setzungen und empirische Befunde der jeweiligen Fachdidaktik auf. Er dient dem Aufbau fachdidaktischen Wissens, insbesondere in Bezug auf lernförderliche Unterrichtsarrangements und Rückmeldungen (vgl. Abb. 1), sowie der Elaboration fachbezogener Überzeugungen. Ziel ist die Förderung eines „evaluativist level“ (Kuhn 2009; s. o.).

Der letzte Punkt macht deutlich, dass im Rahmen der geplanten Intervention nicht nur bei den Studierenden, sondern auch seitens der Lehrenden aus der Fachdidaktik normative Vorstellungen ins Spiel kommen, die mit der „Philosophie“ eines Schulfaches (Bromme 1992) verknüpft sind. Entsprechende Vorstellungen prägen die Fachdidaktiken als normsetzende Wissenschaften. In der Literaturdidaktik setzt sich beispielsweise zunehmend die Auffassung durch, dass beim literarischen Lernen das subjektive Angesprochensein des Lesers und die genaue Wahrnehmung des Textes aufeinander zu beziehen sind (Spinner 2006; Winkler 2015b; Zabka 2015). Was übergeordnete Ziele von Mathematikunterricht betrifft, erweisen sich in der aktuellen Mathematikdidaktik u. a. die von Winter (1996) beschriebenen drei Grunderfahrungen (Mathematik als spezifische Sichtweise auf die Welt; Mathematik als eigenständige, deduktiv geordnete Welt; Beitrag der Mathematik zur Problemlösefähigkeit) als einflussreich, die Mathematikunterricht seiner Meinung nach zu eröffnen habe. Das Wort ,Erfahrung' macht bereits deutlich, dass es Winter nicht nur um das Abspeichern und Hervorrufen von auswendig gelernten Informationen geht. Mathematik soll erlebt werden.

Die beiden folgenden Teilkapitel konkretisieren aus Sicht der Mathematik- und der Deutschdidaktik Ideen, wie über die Reflexion fremder Perspektiven auf das eigene Fach fachdidaktischer Kompetenzerwerb unterstützt werden soll. Ausgangspunkt der Überlegungen sind jeweils simplifizierende Alltagsvorstellungen zum Fach, die sich in der Pilotstudie (siehe Abschnitt 1) ergeben haben. 


\subsection{Mathematikdidaktische Perspektive}

Die Vorstellung, dass Mathematikunterricht auf das bloße Anwenden von Algorithmen abzielt, ist in den Aussagen der Deutschstudierenden aus der Pilotstudie (siehe Abschnitt 1) sehr dominant, z. B.:

- "In Mathe geht es meist um Formeln und die Anwendung dieser."

- „In der Schule hatte ich den Eindruck, dass es im Mathematikunterricht hauptsächlich um das Erlernen von festen Regeln geht, wie man an bestimmte Aufgaben herangeht. Für mich ging es lediglich darum zu erkennen, welcher Aufgabentyp vorliegt und dann das entsprechende ,Kochrezept' abzurufen."

- „In der Schule ist Mathematik bloßes Algorithmen anwenden.“

- „Mathematik ist ein Fach, bei dem alles nur nach Schema A gelöst wird. Man bekommt eine Aufgabe und löst diese mit einer vorgegebenen Formel. Es bleibt kein Platz mehr für Freiraum und Phantasie.“

Nach der Rekonstruktion der Vorstellung von Mathematikunterricht, die aus den Aussagen der Deutschstudierenden spricht, ist mit den Mathematikstudierenden zu diskutieren, wie diese Vorstellung zustande kommt. Vermutlich teilen sie selbst Erinnerungen an einen Mathematikunterricht, in dem den Lernenden feststehende Algorithmen vermittelt werden. Die Lehrperson bringt den Lernenden beispielsweise ein Verfahren bei, wie man bei einer quadratischen Gleichung der Form $x^{2}+7 x-5=0$ das $\mathrm{x}$ bestimmen kann, und dieses Verfahren wird danach in einer Vielzahl von Übungsaufgaben angewendet und eingeübt, bevor zum nächsten Problem übergegangen wird, das durch ein neues Verfahren gelöst werden kann.

Im nächsten Schritt geht es um die Problematisierung dieser Vorstellung. Dabei kann man an die durchaus negative Bewertung des schematischen Vorgehens aus Sicht der zitierten Deutschstudierenden anknüpfen („,kein Platz mehr für Freiraum und Phantasie"). Aus mathematikdidaktischer Sicht besteht das Ziel von Mathematikunterricht nicht darin, am Ende die eine richtige Lösung gefunden zu haben. Knüpft man an die von Winter (1996) beschriebenen mathematischen Grunderfahrungen an, dann geht es beim Auflösen der quadratischen Gleichung nicht vorrangig darum, was das $\mathrm{x}$ in der Gleichung ist, sondern darum, wie man an das $\mathrm{x}$ herankommt. ${ }^{2}$ Wie nähert man sich einem Problem, das zunächst unlösbar scheint? Die Lernenden können es beispielsweise in Teilprobleme zerlegen und

2 Die dritte Grunderfahrung ermöglicht, „, in der Auseinandersetzung mit Aufgaben Problemlösefähigkeiten, die über die Mathematik hinausgehen (heuristische Fähigkeiten), zu erwerben“" (Winter 1996, S. 37). Es stehen also der Erwerb von Heuristiken und deren flexibler Einsatz im Vordergrund (vgl. auch Niedersächsisches Kultusministerium 2006, 
sich diesen dann einzeln widmen. Ebenfalls wäre es denkbar, sich die gegebene Situation zunächst mit Hilfe von Beispielen oder Skizzen zu veranschaulichen oder aber nicht vorne am Problem anzufangen, sondern sich von der vermuteten Lösung, also von hinten, nach vorne zu arbeiten. Die Liste der möglichen Heuristiken ist sehr lang, und ein Ziel des Mathematikunterrichts ist es, dass Schülerinnen und Schüler lernen, diese flexibel zu benutzen und eben nicht bloß,Kochrezepte anwenden zu können (vgl. z. B. Niedersächsisches Kultusministerium 2006).

Je nachdem, welche Erfahrungen die Mathematikstudierenden mit ihrem Fach haben, müssen sie u. U. erst einmal selbst Gelegenheit erhalten, sich Mathematikaufgaben wie der angeführten quadratischen Gleichung anders als über ,Kochrezepte' zu nähern. Diese Erfahrung ist erforderlich, damit aktuelle Zielvorgaben für den Mathematikunterricht (z. B. Niedersächsisches Kultusministerium 2006; Winter 1996), die deutlich über das alleinige Arbeiten mit Algorithmen hinausgehen, für die Studierenden überhaupt nachvollziehbar sind. Herausforderungen und Potenzial eines solchen Vorgehens im Unterricht gilt es zu diskutieren, z. B. durch theoretisches Durchdenken möglicher Unterrichtsverläufe und durch Analyse von Transkripten oder Videoaufzeichnungen von Mathematikunterricht.

Eine weitere typische Vorstellung, die Deutschstudierende vom Mathematikunterricht haben, bezieht sich auf die Determiniertheit von Lösungswegen:

- „Die Mathematik gibt meist den Lösungsweg vor.“

- „Mathematik lässt im Lösungsweg keinerlei Offenheit zu.“

- „Mathematik ist ein Fach, bei dem alles nur nach Schema A gelöst wird. Es bleibt kein Platz mehr für Freiraum und Phantasie."

Diese Überzeugung knüpft unmittelbar an die obengenannte Alltagsvorstellung zur Anwendung von ,Kochrezepten' an. Wer das Gefühl hat, dass bloß ,Kochrezepte' angewendet werden, hält sicherlich auch den Lösungsweg für festgelegt. Auch hier sollten die Studierenden zunächst selbst reflektieren, wie sich die geäußerten Auffassungen erklären lassen und ob sie den Aussagen der Deutschstudierenden zustimmen würden oder nicht. Welche Vorteile hätte es, wenn es tatsächlich immer nur einen Lösungsweg gäbe? Welche Nachteile? Für welche Aufgaben bzw. Probleme gibt es nur einen Lösungsweg, für welche nicht bzw. bei welchen Aufgaben ist der Lösungsweg offen?

Um den Horizont von Mathematikstudierenden, die von der Eindeutigkeit des Lösungsweges schulmathematischer Aufgaben überzeugt sind, für verschiedene

S. 15f.: Wie kann man sich einem mathematischen Problem nähern und es lösen? Wie können Probleme aus dem Alltag mathematisch aufgefasst und gelöst werden? 
Lösungswege zu öffnen, kann man ihnen den Arbeitsauftrag geben, sich zu einer Aufgabe möglichst viele verschiedene Lösungswege zu überlegen. Auch bietet sich die Arbeit mit Schülerlösungen an, die zeigen, dass Lernende eine Aufgabe auf sehr unterschiedliche Art und Weise lösen (vgl. Sjtus 2002).

\subsection{Deutsch-/literaturdidaktische Perspektive}

Zahlreiche der Aussagen von Mathematikstudierenden zum Fach Deutsch setzen an der Offenheit von Lösungen im Literaturunterricht an. Diese wird - anders als in der Perspektive der Deutschstudierenden - aber negativ beurteilt, weil sie Verunsicherung bei Lernenden, Beliebigkeit und Intransparenz von Bewertungen durch die Lehrperson zur Folge haben kann.

- „Im Deutschunterricht gibt es oft viele Interpretationsmöglichkeiten für eine Aufgabe, wodurch es auch zu Unklarheiten beim Verständnis kommen kann."

- „Deutsch kann schwammig und auch mehrdeutig sein, was zu Missverständnissen führen kann.“

- „Der Deutschunterricht [...] ist meist sehr ,spekulativ‘, es gibt viele Interpretationsmöglichkeiten."

- „Auf jede Frage gibt es hundert verschiedene und ,richtige‘ Antworten zugleich.“

Für Deutschstudierende kann es überraschend sein, dass ein Fachmerkmal, das sie selbst für positiv halten, aus Sicht fachfremder Studierender konträr bewertet wird. Dass es im Literaturunterricht i. d. R. mehrere Deutungen eines Textes gibt, liegt in der systematischen Mehrdeutigkeit literarischer Texte begründet (z. B. Spinner 2006; Zabka 2006). Das bedeutet aber noch lange nicht, dass jede individuell beliebige Verstehensvariante als intersubjektiv tragfähig gelten kann. In dem Literaturunterricht, der die Wahrnehmung der zitierten Mathematikstudierenden prägt, ist es offensichtlich nicht gelungen, Kriterien für die Gültigkeit literaturbezogener Argumentationen aufzustellen. Wenn ausgehend von den Aussagen der Mathematikstudierenden eine Sensibilisierung der Deutschstudierenden für die skizzierte Problemlage erfolgt ist, stehen folgende Fragen im Mittelpunkt des literaturdidaktischen Lehr-Lern-Arrangements: Wie gehen Lehrpersonen im Literaturunterricht mit der Mehrdeutigkeit literarischer Texte um (Analyse von Unterrichtsbeispielen)? Welche Probleme zeigen sich? Welche Alternativen gibt es? Inwieweit lassen sich übergeordnete Kriterien für die Tragfähigkeit von literaturbezogenen Argumenten formulieren? ${ }^{3}$

3 Zusätzlich im Rahmen des fächerübergreifenden Lernens: Kann man vom Mathematikunterricht lernen, was die Einschätzung gültiger Argumentationen angeht? 
Der zuletzt genannte Aspekt ist auch bedeutsam, wenn es um die Bewertung von Schülerarbeiten im Literaturunterricht geht. Auch dieser Bereich, der eng mit dem Problem des Umgangs mit Mehrdeutigkeit zusammenhängt, wird von den Mathematikstudierenden als problematisch erlebt:

- „In Deutsch hing das Ergebnis immer davon ab, was der Lehrer hören wollte.“

- „Ich habe in der Schule immer nicht recht nachvollziehen können, wieso mancher Aufsatz nicht so gelungen war. [...] Die Korrektur einer Deutscharbeit ist eher von subjektiver Natur, also der Meinung des Lehrers entsprechend.“

- „Mehrere Ansätze zum Beispiel beim Interpretieren sind zugelassen, jedoch werden einige persönliche Interpretationen auch verworfen beziehungsweise für schlecht gehalten, was dem eigentlichen Sinn meiner Meinung nach total widerspricht."

Das Nachvollziehen dieser Problemlage kann nicht nur die Motivation der Deutschstudierenden erhöhen, sich mit der Tragfähigkeit von Argumenten bzw. Deutungsansätzen im Literaturunterricht auseinanderzusetzen. Es richtet auch den Blick auf die Frage, wie geeignete Leistungsaufgaben im Literaturunterricht aussehen können, die eine adäquate Balance zwischen Offenheit und Determiniertheit bieten (vgl. dazu bereits Köster 2003).

\section{Bilanz}

Im vorliegenden Kapitel gehen wir von der Hypothese aus, dass sowohl fachdidaktisches Wissen als auch fachbezogene Überzeugungen bei Lehramtsstudierenden positiv beeinflusst werden können, wenn sie sich dem Vertrauten über fremde Perspektiven nähern. Inwieweit entsprechende fachdidaktische Angebote tatsächlich die erwünschten Wirkungen entfalten, ist Gegenstand der im Rahmen des vorgestellten Projekts geplanten Interventionsstudien.

Die interdisziplinäre Zusammenarbeit leitet unser Projekt auf zweifache Weise: Sie rahmt die Untersuchungsfrage, die für alle beteiligten Disziplinen zugleich auch aus fachspezifischer Sicht relevant ist, und sie bestimmt maßgeblich das Untersuchungsdesign. Nur wenn alle Beteiligten neugierig darauf sind, über den fachlichen ,Tellerrand' hinaus zu schauen, und die Bereitschaft mitbringen, sich durch fachfremde Blickwinkel produktiv verunsichern zu lassen, kann ein solches Vorhaben gelingen. 


\section{Literatur}

Baumert, Jürgen/Kunter, Mareike (2011a): Das mathematikspezifische Wissen von Lehrkräften, kognitive Aktivierung im Unterricht und Lernfortschritte von Schülerinnen und Schülern. In: Kunter, Mareike/Baumert, Jürgen/Blum, Werner/Klusmann, Uta/Krauss, Stefan/Neubrand, Michael (Hrsg.): Professionelle Kompetenz von Lehrkräften. Ergebnisse des Forschungsprogramms COACTIV. Münster u. a.: Waxmann, S. 163-192.

Baumert, Jürgen/Kunter, Mareike (2011b): Das Kompetenzmodell von COACTIV. In: Kunter, Mareike/Baumert, Jürgen/Blum, Werner/Klusmann, Uta/Krauss, Stefan/Neubrand, Michael (Hrsg.): Professionelle Kompetenz von Lehrkräften. Ergebnisse des Forschungsprogramms COACTIV. Münster u. a.: Waxmann, S. 29-53.

Blömeke, Sigrid (2011): Teacher Education and Development Study. Learning to Teach (TEDS-LT) - Erfassung von Lehrerkompetenzen in gering strukturierten Domänen. In: Blömeke, Sigrid/Bremerich-Vos, Albert/Haudeck, Helga/ Kaiser, Gabriele/Nold, Günter/Schwippert, Knut/Willenberg, Heiner (Hrsg.): Kompetenzen von Lehramtsstudierenden in gering strukturierten Domänen. Erste Ergebnisse aus TEDS-LT. Münster: Waxmann, S. 7-24.

Blömeke, Sigrid/Kaiser, Gabriele/Lehmann, Rainer (Hrsg.) (2010): Professionelle Kompetenz und Lerngelegenheiten angehender Mathematiklehrkräfte für die Sekundarstufe I im internationalen Vergleich. Münster: Waxmann.

Bremerich-Vos, Albert/Dämmer, Jutta (2013). Professionelles Wissen im Studienverlauf: Lehramt Deutsch. In: Blömeke, Sigrid/Bremerich-Vos, Albert/ Kaiser, Gabriele/Nold, Günter/Haudeck, Helga/Keßler, Jörg-U./Schwippert, Knut (Hrsg.): Professionelle Kompetenzen im Studienverlauf - Weitere Ergebnisse zur Deutsch-, Englisch- und Mathematiklehrerausbildung aus TEDS-LT. Münster u. a.: Waxmann, S. 47-75.

Bremerich-Vos, Albert/Dämmer, Jutta/Willenberg, Heiner/Schwippert, Knut (2011): Professionelles Wissen von Studierenden des Lehramts Deutsch. In: Blömeke, Sigrid/Bremerich-Vos, Albert/Haudeck, Helga/Kaiser, Gabriele/ Nold, Günter/Schwippert, Knut/Willenberg, Heiner (Hrsg.): Kompetenzen von Lehramtsstudierenden in gering strukturierten Domänen. Erste Ergebnisse aus TEDS-LT. Münster u. a.: Waxmann, S. 47-76.

Bromme, Rainer (1992): Der Lehrer als Experte. Zur Psychologie des professionellen Wissens. Bern: Huber.

Buchholtz, Nils/Kaiser, Gabriele (2013): Professionelles Wissen im Studienverlauf: Lehramt Mathematik. In: Blömeke, Sigrid/Bremerich-Vos, Albert/Kaiser, Gabriele/Nold, Günter/Haudeck, Helga/Keßler, Jörg-U./Schwippert, Knut (Hrsg.): Professionelle Kompetenzen im Studienverlauf - Weitere Ergebnisse 
zur Deutsch-, Englisch- und Mathematiklehrerausbildung aus TEDS-LT. Münster u. a.: Waxmann, S. 107-143.

Buchholtz, Nils/Kaiser, Gabriele/Stancel-Piątak, Agnes (2011): Professionelles Wissen von Studierenden des Lehramts Mathematik. In: Blömeke, Sigrid/BremerichVos, Albert/Haudeck, Helga/Kaiser, Gabriele/Nold, Günter/Schwippert, Knut/ Willenberg, Heiner (Hrsg.): Kompetenzen von Lehramtsstudierenden in gering strukturierten Domänen. Erste Ergebnisse aus TEDS-LT. Münster u. a.: Waxmann, S. 101-133.

Büchter, Andreas/Leuders, Timo (2014): Mathematikaufgaben selbst entwickeln. Lernen fördern - Leistung überprüfen. 6. Aufl. Berlin: Cornelsen.

Busse, Vera/Krause, Ulrike-Marie (2015): Addressing cultural diversity: effects of a problem-based intercultural learning unit. Learning Environments Research. Vol. 18. No. 3, pp. 425-452.

Cognition and Technology Group at Vanderbilt (1997): The Jasper project: Lessons in curriculum, instruction, assessment, and professional development. Mahwah, NJ: Erlbaum.

Dalbert, Claudia (1999): Die Ungewissheitstoleranzskala: Skaleneigenschaften und Validierungsbefunde. Hallesche Berichte zur Pädagogischen Psychologie Nr. 1. Halle: Martin-Luther-Universität.

Döhrmann, Martina/Kaiser, Gabriele/Blömeke, Sigrid (2010): Messung des mathematischen und mathematikdidaktischen Wissens. Theoretischer Rahmen und Teststruktur. In: Blömeke, Sigrid/Kaiser, Gabriele/Lehmann, Rainer (Hrsg.): TEDS-M 2008. Professionelle Kompetenz und Lerngelegenheiten angehender Mathematiklehrkräfte für die Sekundarstufe I im internationalen Vergleich. Münster: Waxmann, S. 169-196.

Esser, Frank (2012). Fortschritte und Herausforderungen der komparativen Kommunikationswissenschaft. In: Stark, Birgit/Magin, Melanie/Jandura, Olaf/ Maurer, Marcus (Hrsg.): Methodische Herausforderungen komparativer Forschungsansätze. Köln: von Halem, S. 18-45.

Gallin, Peter/Ruf, Urs/Sitta, Horst (1985): Verbindung von Deutsch und Mathematik. In: Praxis Deutsch. Jg. 12. H. 70, S. 17-27.

Haag, Ludwig/Götz, Thomas (2012): Mathe ist schwierig und Deutsch aktuell. Vergleichende Studie zur Charakterisierung von Schulfächern aus Schülersicht. In: Psychologie in Erziehung und Unterricht. Jg. 59. H. 1, S. 32-46.

Härle, Gerhard (2004): Lenken - Steuern - Leiten. Theorie und Praxis der Leitung literarischer Gespräche in Hochschule und Schule. In: Härle, Gerhard/ Steinbrenner, Marcus (Hrsg.): Kein endgültiges Wort. Die Wiederentdeckung des Gesprächs im Literaturunterricht. Baltmannsweiler: Schneider, S. 107-139. 
Hartinger, Andrea/Fölling-Albers, Maria /Mörtl-Hafizović, Dženana (2005): Die Bedeutung der Ambiguitätstoleranz für das Lernen in situierten Lernbedingungen. Psychologie in Erziehung und Unterricht. Jg. 52. H. 2, S. 113-126.

Kämper-van den Boogaart, Michael (2003): Lesekompetenzen - Hauptsache flexibel. Zu einer Parallele zwischen Literaturdidaktik und empirischer Lesepsychologie. In: Abraham, Ulf/Bremerich-Vos, Albert/Frederking, Volker/Wieler, Petra (Hrsg.): Deutschdidaktik und Deutschunterricht nach PISA. Freiburg i. Br.: Fillibach, S. 26-46.

Knapp, Werner (2001): Alltägliche Argumentation - mathematische Argumentation. Analyse von Dialogen zur Lösung von mathematischen Knobelaufgaben. In: Wirkendes Wort. Jg. 51. H. 1, S. 93-118.

Knapp, Werner/Pfaff, Harald (2008): Wie weit kommt Herr Bauer mit einer Tankfüllung? Durch Schreiben mathematische Textaufgaben verstehen. In: Praxis Deutsch. Jg. 35. H. 210, S. 26-30.

Köller, Olaf/Baumert, Jürgen/Neubrand, Johanna (2000): Epistemologische Überzeugungen und Fachverständnis im Mathematik- und Physikunterricht. In: Baumert, Jürgen/Bos, Wilfried/Lehmann, Rainer (Hrsg.): Dritte Internationale Mathematik- und Naturwissenschaftsstudie: Mathematische und naturwissenschaftliche Bildung am Ende der Schullaufbahn. Bd. 2. Mathematische und physikalische Kompetenzen am Ende der gymnasialen Oberstufe. Opladen: Leske + Budrich, S. 229-270.

Köster, Juliane (2003): Konstruieren statt Entdecken. Impulse aus PISA für die deutsche Aufgabenkultur. In: Didaktik Deutsch. Jg. 8. H. 14, S. 4-20.

Köster, Juliane (im Druck): Aufgaben im Deutschunterricht. Wirksame Lernangebote und Erfolgskontrollen. Seelze: Klett/Kallmeyer.

Krause, Ulrike-Marie (2007): Feedback und kooperatives Lernen. Münster: Waxmann.

Krause, Ulrike-Marie/Stark, Robin (2010): Reflection in example- and problembased learning: effects of reflection prompts, feedback, and cooperative learning. In: Evaluation \& Research in Education. Vol. 23. No. 4, pp. 255-272.

Krause, Ulrike-Marie/Stark, Robin/Herzmann, Petra (2011): Förderung anwendbaren Theoriewissens in der Lehrerbildung: Vergleich problembasierten und instruktionsorientierten Lernens. In: Psychologie in Erziehung und Unterricht. Jg. 58. H. 2, S. 106-115.

Krause, Ulrike-Marie/Stark, Robin/Mandl, Heinz (2009): The effects of cooperative learning and feedback on e-learning in statistics. In: Learning and Instruction. Vol. 19. No. 2, pp. 158-170. 
Kuhn, Deanna (2009): The importance of learning about knowing: Creating a foundation for development of intellectual values. In: Child Development Perspectives. Vol. 3. No. 2, pp. 112-117.

Laschke, Christin/Döhrmann, Martina (2014): Beispielitems zur Erhebung des mathematischen und mathematikdidaktischen Wissens. In: Laschke, Christin/ Blömeke, Sigrid (Hrsg.): Teacher Education and Development Study: Learning to Teach Mathematics (TEDS-M 2008). Dokumentation der Erhebungsinstrumente. Münster: Waxmann, S. 347-409.

Laschke, Christin/Kaiser, Gabriele (2014): Beispielitems zur Erhebung des mathematischen und mathematikdidaktischen Wissens. In: Laschke, Christin/ Blömeke, Sigrid (Hrsg.): Teacher Education and Development Study: Learning to Teach Mathematics (TEDS-M 2008). Dokumentation der Erhebungsinstrumente. Münster: Waxmann, S. 131-226.

Maier, Uwe/Bohl, Thorsten/Drüke-Noe, Christina/Hoppe, Henriette/Kleinknecht, Marc/Metz, Kerstin (2014): Das kognitive Anforderungsniveau von Aufgaben analysieren und modifizieren können: Eine wichtige Fähigkeit von Lehrkräften bei der Planung eines kompetenzorientierten Unterrichts. In: Beiträge zur Lehrerinnen- und Lehrerbildung. Jg. 32. H. 3, S. 340-358.

Niedersächsisches Kultusministerium (2006): Kerncurriculum für das Gymnasium. Schuljahrgänge 5-10. Mathematik. Hannover: Unidruck.

Reinmann, Gabi/Mandl, Heinz (2006): Unterrichten und Lernumgebungen gestalten. In: Krapp, Andreas/Weidenmann, Bernd (Hrsg.): Pädagogische Psychologie. Weinheim: Beltz, S. 613-658.

Schmeisser, Christine/Krauss, Stefan/Bruckmaier, Georg/Ufer, Stefan/Blum, Werner (2013): Transmissive and constructivist beliefs of in-service mathematics teachers and of beginning university students. In: Li, Yeping/Moschkovich, Judith N. (Eds.): Proficiency and beliefs in learning and teaching mathematics. Learning from Alan Schoenfeld and Günter Törner. Rotterdam, Boston, Taipei: Sense Publishers, pp. 51-67.

Schmotz, Christiane/Felbrich, Anja/Kaiser, Gabriele (2010): Überzeugungen angehender Mathematiklehrkräfte für die Sekundarstufe I im internationalen Vergleich. In: Blömeke, Sigrid/Kaiser, Gabriele/Lehmann, Rainer (Hrsg.): TEDS-M 2008. Professionelle Kompetenz und Lerngelegenheiten angehender Mathematiklehrkräfte für die Sekundarstufe I im internationalen Vergleich. Münster: Waxmann, S. 279-305.

Schoenfeld, Alan H. (1992): Learning to think mathematically: Problem solving, metacognition, and sense-making in mathematics. In: Grouws, Douglas (Ed.): Handbook for Research on Mathematics Teaching and Learning. New York: MacMillan, S. 334-370. 
Schriewer, Jürgen (2003): Problemdimensionen sozialwissenschaftlicher Komparatistik. In Kaelble, Hartmut /Schriewer, Jürgen (Hrsg.): Vergleich und Transfer. Komparatistik in den Sozial-, Geschichts- und Kulturwissenschaften. Frankfurt a. M./New York: Campus, S. 9-52.

Sjuts, Johann (2002): Unterschiedliche mentale Konstruktionen beim Aufgabenlösen. Eine Fallstudie zur Mathematik als Werkzeug zur Wissenspräsentation. In: Journal für Mathematik-Didaktik. Jg. 23. H. 2, S. 106-128.

Spinner, Kaspar H. (2006): Literarisches Lernen. In: Praxis Deutsch. H. 200, S. 6-16.

Spiro, Rand J./Collins, Brian P./Thota, Jose Jagadish/Feltovich, Paul J. (2003): Cognitive flexibility theory: hypermedia for complex learning, adaptive knowledge application, and experience acceleration. In: Educational Technology. Vol. 43. No. 5, pp. 5-10.

Spiro, Rand J./Feltovich, Paul J./Jacobson, Michael J./Coulson, Richard L. (1992): Cognitive flexibility, constructivism, and hypertext: Random access instruction for advanced knowledge acquisition in ill-structured domains. In: Duffy, Thomas M./Jonassen, David H. (Eds.): Constructivism and the technology of instruction. Hillsdale, NJ: Lawrence Erlbaum, pp. 57-75.

Stark, Robin (2000): Experimentelle Untersuchungen zur Überwindung von Transferproblemen in der kaufmännischen Erstausbildung. In: Zeitschrift für Pädagogik. Jg. 46. H. 3, S. 395-415.

Stark, Robin/Gruber, Hans/Mandl, Heinz/Hinkofer, Ludwig (2001): Implementing example-based learning and teaching models to foster action competence in the domain of economics: an experimental study and first evaluation results in vocational school. Paper presented at the annual meeting of the American Educational Research Association (AERA), Seattle.

Stark, Robin/Mandl, Heinz/Gruber, Hans/Renkl, Alexander (1999): Instructional means to overcome transfer problems in the domain of economics: Empirical studies. In: International Journal of Educational Research. Vol. 31. No. 7, pp. 591-609.

Sweller, John (2010): Element interactivity and intrinsic, extraneous, and germane cognitive load. In: Educational Psychology Review. Vol. 22. No. 2, pp. 123-138.

Tainio, Liisa/Winkler, Iris (2014): The construction of ideal reader in German and Finnish textbooks for literacy education. In: L1 - Educational Studies in Language and Literature. Vol. 14, pp. 1-25.

Vogt, Rüdiger (2004): Symbolische Textdeutungen entwickeln: Lehrer und Schüler interpretieren Peter Hacks' Geschichte „Der Bär auf dem Försterball“ im Unterrichtsgespräch. In: Härle, Gerhard/Steinbrenner, Marcus (Hrsg.): Kein 
endgültiges Wort. Die Wiederentdeckung des Gesprächs im Literaturunterricht. Baltmannsweiler: Schneider, S. 241-264.

Voss, Thamar/Kleickmann, Thilo/Kunter, Mareike/Hachfeld, Axinja (2011): Überzeugungen von Mathematiklehrkräften. In: Kunter, Mareike/Baumert, Jürgen/ Blum, Werner/Klusmann, Uta/Krauss, Stefan/Neubrand, Michael (Hrsg.): Professionelle Kompetenz von Lehrkräften. Ergebnisse des Forschungsprogramms COACTIV. Münster u. a.: Waxmann, S. 235-258.

Weinert, Franz E. (2001): Vergleichende Leistungsmessung in Schulen - eine umstrittene Selbstverständlichkeit. In: Ders. (Hrsg.): Leistungsmessungen in Schulen. Weinheim/Basel: Beltz, S. 17-31.

Willenberg, Heiner (2002): Die Evaluation des Leseverständnisses durch die PISA-Studie und der Literaturunterricht in der Sekundarstufe I. In: Deutschunterricht. Jg. 55. H. 3, S. 39-45.

Winkler, Iris (2010): Lernaufgaben im Literaturunterricht. In: Kiper, Hanna/ Meints, Waltraud/Peters, Sebastian/Schlump, Stephanie/Schmit, Stefan (Hrsg.): Lernaufgaben und Lernmaterialien im kompetenzorientierten Unterricht. Stuttgart: Kohlhammer, S. 103-113.

Winkler, Iris (2015a): Durch die Brille der anderen sehen. Professionsbezogene Überzeugungen im Lehramtsstudium Deutsch. In: Mitteilungen des Deutschen Germanistenverbandes. Jg. 62. H. 2, S. 192-208.

Winkler, Iris (2015b): „Subjektive Involviertheit und genaue Wahrnehmung miteinander ins Spiel bringen“. Überlegungen zur Spezifikation eines zentralen Konzepts für den Literaturunterricht. In: Leseräume. H. 2, S. 155-168.

Winter, Heinrich (1996): Mathematikunterricht und Allgemeinbildung. In: Mitteilungen der Gesellschaft für Didaktik der Mathematik. H. 61, S. 37-46.

Zabka, Thomas (2006): Typische Operationen literarischen Verstehens. Zu Martin Luther, Vom Raben und Fuchs (5./6. Schuljahr). In: Kammler, Clemens (Hrsg.): Literarische Kompetenzen - Standards im Literaturunterricht. Modelle für die Primar- und Sekundarstufe. Seelze: Kallmeyer, S. 80-101.

Zabka, Thomas (2012). Analyserituale und Lehrerüberzeugungen. Theoretische Untersuchung vermuteter Zusammenhänge. In: Pieper, Irene/Wieser, Dorothee (Hrsg.): Fachliches und literarisches Wissen und Verstehen. Studien zu einer brisanten Relation. Frankfurt a. M. et al.: Lang, S. 35-52.

Zabka, Thomas (2015): Was ist Hochschulreife im Umgang mit Literatur? In: Didaktik Deutsch. H. 38, S. 136-150. 
Iris Winkler and Frederike Schmidt - 978-3-631-69285-1

Downloaded from PubFactory at 01/11/2019 10:32:53AM

via free access 


\author{
Frederike Schmidt, Barbara Moschner
}

\title{
„Fremde Schwestern“ und „kritische Freundinnen" - Lehren und Lernen in einem interdisziplinären Promotionsprogramm in der Lehrerbildung
}

\begin{abstract}
In this article we discuss how the perspectives of research groups from several disciplines, including „Deutschdidaktik“, are interlocked in an interdisciplinary Graduate Program. The Graduate Program Processes of Educational Structuring for Classroom Practice and Teacher Education (ProfaS) focuses on the study of this professional knowledge as well as on teachers' subjective theories. Results of these empirical research studies are combined with domain-specific teaching approaches in order to develop guidelines for teacher education. The paper will show that the individual domains benefit from interdisciplinary processes in the program: the multiple representations of teaching and learning provide new perspectives for disciplinary thinking. In addition the perspectives from other domains foster the reflection of the domain-specific point of view.
\end{abstract}

\section{Interdisziplinär lehren und lernen. Oder: Wie führt man Denkstile zusammen?}

Naturwissenschaftler, Philologen, Theologen oder Kabbalisten können sich innerhalb ihrer Gemeinschaften ausgezeichnet verständigen, aber die Verständigung eines Physikers mit einem Philologen ist schwierig, mit einem Theologen sehr schwierig und mit einem Kabbalisten oder Mystiker unmöglich. (Fleck 1936, S. 263)

Flecks erkenntnistheoretische Überlegungen erfolgten zu einer Zeit, in der disziplinübergreifende Arbeitsgruppen und Forschungsprojekte nicht so zahlreich vorhanden waren wie heute. Dass interdisziplinäre Kooperationen in der Deutschdidaktik längst nicht mehr „nur durch den Aufweis von [disziplinären] Defiziten [zu] rechtfertigen“ sind (Kocka 1987, S. 8), sondern zunehmend „ein ernstes wissenschaftssystematisches Thema" (Mittelstraß 2012, S. 11) in Forschung und Praxis darstellen, spiegeln nicht zuletzt die Beiträge im vorliegenden Sammelband wider. Gleichwohl wird hier im Kern eine wesentliche Erkenntnisbedingung deutlich, wenn es darum geht, dass Deutschdidaktikerinnen und 
Deutschdidaktiker mit Forschenden aus anderen Fächern ${ }^{1}$ kooperieren wollen: In disziplinübergreifenden Forschungsverbünden treffen, wie Fleck (1935, S. 130) in seiner Erkenntnistheorie dargestellt hat, die Denkstile verschiedener wissenschaftlicher Denkkollektive aufeinander. Unser Denkstil beeinflusst die Perspektive, die wir auf Gegenstände einnehmen und die Wahrnehmung und Setzungen dessen, was von uns als zentral für Forschung erachtet wird: „Was wir denken und wie wir sehen [und interpretieren], hängt von dem Denkkollektiv ab, dem wir angehören“ (ebd., S. 233). Flecks Theorie von Denkstilen und Denkkollektiven lässt sich mit Gewinn auf fächerübergreifende Forschungskonstellationen beziehen: Die Beteiligten müssen einen Weg finden, die einzelnen Denkstile im Kooperationsprozess integrativ zusammenzuführen, um zu einer "tatsächlichen Zusammenarbeit" (Terhart 2002, S. 77) und „wirkliche[n] Auseinandersetzung zwischen den Disziplinen“ (Prediger 2003, S. 7) zu gelangen. Diese Herausforderung ist auch und besonders in fachübergreifenden Promotionsprogrammen zu bewältigen, in denen nicht nur zwei, sondern eine Vielzahl an unterschiedlichen Disziplinen in einen Forschungsdialog treten und das Kooperationsformat gleichzeitig einen Ort der wissenschaftlichen Ausbildung für Promovierende darstellt.

In diesem Beitrag möchten wir Bedingungen und Erkenntnisse dieser Form interdisziplinärer Vernetzung anhand eigener Erfahrungen diskutieren, die wir als Deutschdidaktikerin/Doktorandin und als empirische Lehr-Lernforscherin/ Betreuerin - in dem Oldenburger Promotionsprogramm „Prozesse fachdidaktischer Strukturierung (ProfaS)“ gesammelt haben. Unsere Ausgangsthese ist, dass in der gemeinsamen Forschungsarbeit die jeweiligen Einzeldisziplinen von den kooperierenden Aktivitäten profitieren können. Gelingt derartige Forschung, schärfen und erweitern alle Beteiligten nicht nur ihren disziplinären Blick, sondern erwerben zudem „ein spezifisches Wissen und Können, das über reines Faktenwissen und bloße Sachkompetenz hinausgeht" (Defila/Di Giulio 1998, S. 119).

\section{Außenansichten: Herausforderungen interdisziplinären Arbeitens in einem fachübergreifenden Promotionsprogramm}

Noch im Jahr 2011 hat Ewald Terhart konstatiert, dass im Verhältnis von „Erziehungswissenschaft und Fachdidaktik [...] insgesamt wenig Kommunikation und Kooperation“ bestand und die „Fachdidaktiken auch untereinander [...]

1 Auf die Unterscheidung von „Fach“ und „Disziplin“ gehen wir im vorliegenden Beitrag nicht vertiefend ein (vgl. zu dieser Auseinandersetzung z. B. Heckhausen 1987, S. 129-131). Wir verwenden beide Begriffe nachfolgend synonym. 
selten Beziehungen aufbauten" (Terhart 2011, S. 245). ${ }^{2}$ Dass sich die genannten Disziplinen oftmals mit dem gemeinsamen Diskurs schwer tun und erst in den letzten Jahren zunehmend miteinander im Gespräch sind, ist darauf zurückzuführen, dass es „besonderer Anstrengungen“ (Mittelstraß 2012, S. 11) bedarf, wenn sich Fachdidaktiken und Bildungswissenschaften in einen gemeinsamen Forschungsprozess begeben. Auf die Bedingungen, die für die Umsetzung und das Gelingen interdisziplinärer Forschung in der Deutschdidaktik (mit) entscheidend sind, wollen wir in einem ersten Schritt näher eingehen und zwei wesentliche Herausforderungen diskutieren, die in unserem Promotionsprogramm zu bewältigen waren. ${ }^{3}$

\subsection{Aushalten der Vielfalt: Pluralität der Zugänge zu Lehr- und Lernprozessen im Unterricht}

Das Promotionsprogramm ProfaS führte über vier Jahre Vertreter der Deutschdidaktik, anderer Fachdidaktiken und den Bildungswissenschaften zusammen (s. Abschnitt 3.1). In der vergleichenden Betrachtung wird deutlich, dass sich die einzelnen Disziplinen nicht nur hinsichtlich ihrer Begrifflichkeiten (s. Abschnitt 2.2), sondern auch in anderen Bereichen wie ihren epistemologischen Überzeugungen, Herangehensweisen und ihrem Wissenskanon unterscheiden. ${ }^{4}$ Dass es aber nicht nur Trennendes, sondern auch stark Verbindendes im Beziehungsgefüge von Bildungswissenschaften und den einzelnen Fachdidaktiken gibt, macht Ewald Terhart in seinen Ausführungen deutlich:

Thematisch und von den Aufgaben in Forschung und Lehre her liegen Schulpädagogik, Allgemeine Didaktik, Unterrichtsforschung und Fachdidaktik sehr eng zusammen: Ihr Gegenstandsfeld ist das Lehren und Lernen in der Schule, z. T. auch außerhalb dieser. [...] Ausgangspunkt, Blickrichtung und Akzentsetzung hinsichtlich des Gegenstandes sind in Teilen jedoch different. (Terhart 2011, S. 255f.)

Es drängen sich also ähnliche Grundfragen in Forschung und Lehre auf, anhand derer sich die Disziplinen einander annähern können. Die Rekonstruktion von Lernprozessen und die reflexive Beobachtung von Unterricht gehören zum Kern aller Fächer in der Bildungsforschung. Durch das geteilte Gegenstandsfeld sind

2 Dass der fehlende Diskurs zudem hinsichtlich der gemeinsamen Aufgabe(n) in der Lehramtsausbildung Schwierigkeiten aufwirft, kann hier nur knapp angeführt werden (vgl. dazu u. a. Reusser 2008, S. 222).

3 Im Hinblick auf weitere personelle, organisatorische und strukturelle Voraussetzungen interdisziplinärer Zusammenarbeit vgl. u. a. Defila/Di Giulio (1998).

4 Vgl. vertiefend zu diesen Bestandteilen einer Disziplin Prediger (2003, S. 4ff.). 
die einzelnen Disziplinen per se eng(er) aufeinander bezogen, weshalb Terhart (2002) in einem anderen Beitrag von „Familienverhältnisse[n]“ (ebd., S. 81) spricht, wenn er das Beziehungsgefüge von Allgemeiner Didaktik und empirischer Lehr-Lernforschung diskutiert, und Kurt Reusser (2008) dieses Familienbild einige Jahre später noch um die Fachdidaktiken erweitert.

Metaphorisch lassen sich die einzelnen fachlichen Zugänge als Lupen im Kontext von Lehren und Lernen begreifen, die unterschiedliche Ausschnitte einer komplexen Realität zu erfassen versuchen - und dabei gleichzeitig andere Aspekte ausblenden (müssen). Hier wird konkret, was Fleck (1935) unter „gerichtetem Wahrnehmen" bedingt durch den jeweiligen Denkstil fasst (vgl. ebd., S. 130). Gleichzeitig kann und sollte man dies auch als Chance begreifen: Gerade dort, wo es sich um verschiedene und dennoch jeweils nah verwandte Disziplinen handelt, wird ein konstruktiver interdisziplinärer Dialog wahrscheinlicher (vgl. Sukopp 2010, S. 23). Für die Wirksamkeit von Multiperspektivität in Forschungskontexten - in unserem Fall in Bezug auf Kompetenz fördernde Lehrerbildung und Unterrichtsentwicklung - ist gleichwohl relevant, dass bei den Einzelnen eine „kulturelle Bewusstheit“ (Prediger 2003, S. 8) und damit Relativität des eigenen Denkstils besteht: „Es gilt, [...] die eigene Disziplin als spezifische Sicht auf die Welt, als eine Kultur unter vielen möglichen“ zu sehen (Defila/Di Giulio 1998, S. 125). Diese (eigen-)kulturelle Bewusstheit für die interdisziplinäre Verständigung verweist zugleich auf eine weitere wesentliche Herausforderung, wenn eine wirkliche Auseinandersetzung zwischen den Disziplinen erfolgen soll: die interdisziplinäre Kommunikation zwischen den Fachkulturen.

\subsection{Wovon reden wir eigentlich? Über Kommunikation zum interdisziplinären Verstehen}

In fächerübergreifenden Forschungskooperationen ist die Kommunikation besonders bedeutungsvoll, da sie als Produkt und Medium des Erkenntnisprozesses im interkollektiven Austausch anzusehen ist. Bromme (1999, S. 41) kennzeichnet die Kommunikation deshalb sogar als „Kern der Interdisziplinarität“. Welche Herausforderungen und Dynamiken in sprachlicher Hinsicht bestehen, wenn Vertreter aus mindestens zwei unterschiedlichen Disziplinen miteinander im Gespräch sind, lässt sich wiederum unter Rückgriff auf Flecks Erkenntnistheorie konkretisieren:

Ein besonderes interkollektives Verkehrsgut bildet das Wort als solches: da allen Worten eine mehr oder weniger ausgeprägte denkstilgemäße Färbung anhaftet, die sich bei der interkollektiven Wanderung ändert, kreisen sie interkollektiv immer mit einer gewissen Änderung ihrer Bedeutung. Man vergleiche die Bedeutung der Worte »Kraft« oder 
»Energie« oder »Versuch« für einen Physiker und für einen Philologen oder Sportsmann. Oder das Wort »erklären « für einen Philosophen und für einen Chemiker, oder »Strahl« für einen Künstler und einen Physiker, »Gesetz« für einen Juristen und einen Naturforscher usw. (Fleck 1935, S. 143)

Flecks Beschreibung macht deutlich, was im Wissenschaftskontext gelegentlich übersehen wird: Die Mitglieder eines Denkkollektivs verfügen über gemeinsame epistemologische Überzeugungen und Methoden und über ein geteiltes sprachliches Wissen, welches expliziter Ausdruck des Denkstils einer Disziplin ist. Das bedeutet: Ein gemeinsames Verständnis bestimmter Termini kann im interdisziplinären Dialog nicht vorausgesetzt werden. Darüber hinaus gibt es Teile des Wissens einer Disziplin, die nur selten expliziert werden. Sie basieren auf unhinterfragten, geteilten Überzeugungen. Unter Rückgriff auf Polanyi bezeichnet Susanne Prediger (2003) diese Wissensbestände als implizites Wissen einer Disziplin: es ist „,unabdingbar, um die entsprechende Wissenschaft zu betreiben“ (ebd., S. 5). So ist etwa die deutschdidaktische Debatte im Hinblick auf die Zielsetzungen des Literaturunterrichts mit normativen Fragen zur Interaktion von Leser und literarischem Text als grundlegendem Prinzip des Textverstehens verschränkt, ohne dass dies immer offengelegt wird (vgl. Winkler 2015).

In der Verständigung zwischen einzelnen Disziplinen können die beschriebenen Aspekte zu Verständnis- und ,Übersetzungsproblemen' führen, welche die gemeinsame Forschungsarbeit erschweren (vgl. allgemein Kocka 1987, S. 9; Sukopp 2010, S. 15). ${ }^{5}$ So wurde die von Fleck beschriebene „denkstilgemäße Färbung“ (s. o.) zu Beginn unseres Promotionsprogramms etwa anhand des komplexen Begriffes der „Fachdidaktischen Strukturierung“ (s. Abschnitt 3.2) und fachübergreifend stark diskutierten Termini wie „Aufgabe“ oder „Interesse“ deutlich. Konstruktiver interdisziplinärer Austausch kann aber nur über eine gemeinsame Sprache erfolgen (vgl. u. a. Immelmann 1987, S. 87; Bromme 1999, S. 47). Daher musste der Prozess der sprachlichen Auseinandersetzung selbst stärker in das Blickfeld unseres Promotionsprogramms rücken: Die Differenzerfahrungen der Perspektiven wurden in der gemeinsamen Forschungsarbeit diskutiert und so vertraute Wege der disziplinären Kommunikation verlassen, um überhaupt eine wechselseitige Verständigung zu ermöglichen. Für uns Promovierende und Lehrende bestand im Verlauf des Programms die Herausforderung insofern darin,

den Prozess des Expertiseerwerbs während der Kommunikation zumindest teilweise temporär rückgängig zu machen. Das Wissen muss ,entpackt' werden, die scheinbar

5 Eng verknüpft mit diesem Aspekt ist die wiederholt formulierte Forderung, eine gemeinsame Fachsprache in den Fachdidaktiken zu entwickeln (vgl. z. B. Reusser 2008, S. 222). 
selbstevidente komplexe Situationswahrnehmung muss expliziert werden. (Bromme/ Jucks/Rambow 2004, S. 182)

Mit Bromme (1999) lässt sich diese Sicherung des Verständigungsprozesses als "grounding “6 kennzeichnen. Im Rahmen des grounding-Prozesses war es wiederholt notwendig, die eigene Disziplin in Vergleichsrelation zu anderen Fächern zu setzen, um disziplinäre Erkenntnisse für Fachfremde zu verdeutlichen. Eine klar interdisziplinäre Haltung wird hier deutlich, die zugleich ein hohes Reflexionsniveau mit Blick auf die Kommunikationspartner einfordert (vgl. ähnlich Defila/ Di Giulio 1998, S. 119; Mittelstraß 2012, S. 12).7

Um die in diesem Abschnitt skizzierten Herausforderungen interdisziplinärer Wissenschaftspraxis zu bewältigen, müssen vor allem auch angemessene Forschungsstrukturen geschaffen werden. Wie dieser Anspruch im Kontext unseres fächerübergreifenden Promotionsprogramms eingelöst wurde, soll nachfolgend erörtert werden.

\section{Innenansichten: Die Oldenburger Tradition interdisziplinärer Promotionsprogramme in den Fachdidaktiken und Bildungswissenschaften}

Im Promotionsprogramm ProfaS arbeiteten insgesamt 21 Promovierende und 10 Betreuerinnen und Betreuer aus folgenden disziplinären Kontexten zusammen: Empirische Lehr- und Lernforschung, Deutschdidaktik, Biologiedidaktik, Physikdidaktik, Mathematikdidaktik, Chemiedidaktik, Geschichtsdidaktik, Didaktik der Informatik und die Didaktik des Sachunterrichts. Welche gemeinsame Forschungsfrage für Vertreter dieser Disziplinen besonders tragfähig ist, hat Kurt Reusser in einem weitblickenden Beitrag hervorgehoben:

In der design-basierten Forschungszusammenarbeit interdisziplinärer Gruppen von Lehr-Lern-Forschern und Allgemein- und Fachdidaktikern sehe ich das größte Potenzial, den bisher nur zögerlich stattfindenden Dialog zwischen den beiden Wissenschafts- und

6 Den Begriff des grounding verwendet Bromme (1999, S. 46f.) im Rückgriff auf Clark (1996) und bezeichnet damit die gegenseitige Rückversicherung von Kommunikationspartnern, dass die Nachricht richtig verstanden wurde. Dieser Prozess der Wissensintegration wird von Bromme als eine wesentliche Voraussetzung für das Verstehen in der interdisziplinären Zusammenarbeit diskutiert.

7 Unter anderem geht es auch darum, anzunehmen, dass die Rolle des Experten in der interdisziplinären Konstellation immer wieder wechselt (vgl. Bromme 1999; Bromme/ Jucks/Rambow 2004). 
Disziplinwelten, sowie allgemeiner zwischen Disziplin und Profession zu stärken. (Reusser 2008, S. 233)

Den von Reusser beschriebenen „neuen Forschungstypus“ (ebd.) praktizieren die Bildungswissenschaften und Fachdidaktiken an der Universität Oldenburg schon seit mehreren Jahren, sodass hier in gewisser Weise eine Tradition fachübergreifender Promotionsprogramme in der Bildungsforschung besteht. Bereits viermal konnten seit dem Jahr 2001 erfolgreich Drittmittel für ein entsprechendes Oldenburger Promotionsprogramm eingeworben werden, wie der folgende Überblick knapp verdeutlichen soll:

ProDid 1 (Promotionsprogramm Didaktische Rekonstruktion) 2001-2004

- 15 Georg-Christoph-Lichtenberg-Stipendiaten

- 15 weitere Doktoranden der Universitäten Oldenburg und Bremen

ProDid 2 (Promotionsprogramm Didaktische Rekonstruktion) 2005-2009

- 12 Georg-Christoph-Lichtenberg-Stipendiaten

- 12 weitere Doktoranden der Universitäten Oldenburg und Bremen

ProfaS (Prozesse fachdidaktischer Strukturierung) 2010-2014

- 8 Georg-Christoph-Lichtenberg-Stipendiaten

- 13 weitere Doktoranden der Universitäten Oldenburg und Bremen

LÜP (Lernprozesse im Übergangsraum) 2013-2016

- 15 Georg-Christoph-Lichtenberg-Stipendiaten

Der Überblick veranschaulicht, dass wir im Promotionsprogramm ProfaS auf langjährige Erfahrungen interdisziplinärer Kooperation bei mehreren beteiligten Lehrenden unseres Programms aufbauen konnten. Sicherlich waren diese Vorerfahrungen ein wesentlicher Faktor, der die interdisziplinäre Zusammenarbeit in ProfaS positiv befördert hat.

Die Oldenburger Studienprogramme sind strukturell an internationalen, gestuften Graduiertenprogrammen orientiert und inhaltlich an das Programm der Leading-Teacher-Ausbildung am Weizmann Institute in Israel angelehnt (vgl. Hofstein/Carmi/Ben-Zvi 2003). Das Lehrprogramm im Promotionsprogramm umfasst daher theoretische und methodische Schwerpunktsetzungen sowie Wochenendworkshops mit Präsentationen aller Dissertationsprojekte, die zweimal im Jahr durchgeführt werden. Neben der Präsentation der Doktorarbeiten auf nationalen und internationalen Tagungen ist weiterhin ein internationaler Workshop, der mit Gastwissenschaftlern aus verschiedenen Ländern und Fachdisziplinen durchgeführt wird, kennzeichnend für die Oldenburger Promotionsprogramme von Fachdidaktiken und Bildungswissenschaften. Wie bereits mehrfach erwähnt, werden in diesem Beitrag die Erfahrungen und Erkenntnisse aus dem Programm 
ProfaS beleuchtet. Schwerpunkte und Bezugsrahmen dieses Oldenburger Promotionsprogramms sollen nachfolgend knapp diskutiert werden.

\subsection{ProfaS - Grundlagen eines strukturierten Promotions- programms in der Lehrerbildung}

Im Kontext von ProfaS wurde der Fokus auf die professionelle(n) Kompetenz(en) von Lehrkräften gelegt, womit ein bedeutendes Inhaltsgebiet für alle beteiligten Disziplinen zentral gesetzt wurde: Diversen Studien der letzten Jahre zufolge hat die Professionalität von Lehrkräften, $u$. a. ihr fachliches und fachdidaktisches Wissen, einen signifikanten Einfluss auf den Lernfortschritt von Schülerinnen und Schülern (vgl. exemplarisch Baumert/Kunter 2006). Aus dieser Perspektive kommt der Lehrerforschung eine verstärkte Bedeutung $\mathrm{zu}$, die folglich in den letzten Jahren als Gegenstandsfeld größere Berücksichtigung in der Bildungsforschung findet (vgl. u. a. Bräuer/Winkler 2012; Bräuer/Wieser 2015; Terhart/ Bennewitz/Rothland 2014). Welcher Inhaltsbereich der Lehrerforschung besonders tragfähig ist, um die multiplen Perspektiven fachspezifischer Bildungsforschung zusammenzuführen, führt Kurt Reusser in einem Beitrag zur empirisch fundierten Didaktik näher aus:

Bei solchen [interdisziplinären] Projekten sollte es sich [...] um designbasierte Entwicklungsforschungsprojekte handeln, bei denen fachdidaktische Lernumgebungen auf der Basis des besten verfügbaren Erfahrungs- und Forschungswissens von Lehrenden und Forschenden gemeinsam entwickelt, erprobt und evaluiert werden [...]. (Reusser 2008, S. 233)

Kerngedanke in Reussers Ausführungen ist, neben dem disziplinären Wissen auch die sog. „wisdom of practice“ (Shulman 1986) für die Umsetzung von Forschungserkenntnissen und Konzeption von (fach-)didaktischen Innovationen produktiv zu machen. Im Rahmen von ProfaS hat die von Reusser geforderte Forschungsperspektive Anwendung gefunden: Ein entscheidender Ansatz in unserem Promotionsprogramm war es, die Sichtweisen von (angehenden) Lehrkräften und die Perspektive(n) der jeweiligen Disziplin zueinander in Beziehung zu setzen, um Leitlinien und Elemente für die Unterrichtspraxis und Lehrerbildung zu entwickeln (vgl. Komorek/Fischer/Moschner 2013, S. 52).

\subsection{Ein interdisziplinäres (Selbst-) Verständnis finden: Das Modell der Didaktischen Rekonstruktion}

„Damit von Tradition und Herkunft ungleiche Partner zusammenfinden, braucht es eine ,Geschäftsgrundlage “" (Reusser 2008, S. 224). Für die Oldenburger Promotionsprogramme war und ist diese Verständigungsbasis das Modell der Didaktischen 
Rekonstruktion (Kattmann et al. 1997). Das Modell der Didaktischen Rekonstruktion hat sich - vor allem in den naturwissenschaftsdidaktischen Disziplinen als Rahmenmodell für fachdidaktische Forschungs- und Entwicklungsarbeit in vielfältigen Forschungsprojekten sehr bewährt. ${ }^{8}$ Es ist eine "praktische Theorie“ (Kattmann 2007), die sowohl zur Unterrichtsplanung als auch für fachdidaktische Lehr-Lernforschung genutzt werden kann:

Bei der Didaktischen Rekonstruktion eines Unterrichtsgegenstandes werden drei wechselwirkende Teile eng aufeinander bezogen: fachliche Klärung, Erfassung von Schülervorstellungen und didaktische Strukturierung. (Kattmann et al. 1997, S. 4)

Im Rahmen der Didaktischen Rekonstruktion werden fachdidaktische Perspektiven und Schülervorstellungen zu einem bestimmten Themenbereich systematisch zueinander in Beziehung gesetzt und als wesentliche Quellen angesehen, um begründet fachdidaktische Strukturierungen als Grundlage für Unterrichtsdesigns zu entwickeln (vgl. auch Komorek/Fischer/Moschner 2013, S. 44). Mit der Didaktischen Rekonstruktion werden daher wesentliche Aufgaben fachdidaktischer Forschungs- und Entwicklungsarbeiten in ihren wechselseitigen Bezügen, ihren Voraussetzungen und Abhängigkeiten modelliert. Gleichwohl gibt es für Forschungsarbeiten zur Didaktischen Rekonstruktion keine bestimmten Methoden und Setzungen, sodass das Modell flexibel an die jeweilige Fragestellung angepasst werden kann (vgl. Kattmann et al. 1997, S. 16). Das Modell der Didaktischen Rekonstruktion stellt vielmehr einen Denkrahmen dar, der fachdidaktische Forschungsfragen zu systematisieren hilft (s. a. Abschnitt 4.1).

Im Promotionsprogramm ProfaS wurde das Modell der Didaktischen Rekonstruktion auf den Bereich der Lehrerbildung übertragen und zielt auf fachdidaktische Strukturierungen für die Professionalisierung von Lehrkräften (vgl. Komorek/ Fischer/Moschner 2013). ${ }^{9}$ Folgende drei Untersuchungsaufgaben sind im Modell der Didaktischen Rekonstruktion für die Lehrerbildung wesentlich (vgl. Abb. 1):

- „Fachdidaktische Klärung“: Diese Untersuchungsaufgabe umfasst die Analyse fachlicher und fachdidaktischer Konzeptionen zu einem bestimmten Untersuchungsgegenstand. Für die Fachdidaktische Klärung können unterschiedliche

8 So umfasst etwa die Schriftenreihe „Beiträge zur Didaktischen Rekonstruktion“ mittlerweile beinah fünfzig Bände (https://www.uni-oldenburg.de/diz/publikationen/ beitraege-zur-didaktischen-rekonstruktion). Abgerufen am 11.01.2016.

9 Die Entwicklung von Didaktischen Strukturierungen für die Lehrerbildung sind auch im ERTE-Modell (Educational Reconstruction for Teacher Education) zentral (van Dijk/Kattmann 2007), aus dem das Promotionsprogramm ProfaS unter anderem hervorgegangen ist (vgl. Komorek/Fischer/Moschner 2013, S. 53). 
Quellen wie Lehrbücher, fachliche und fachdidaktische Publikationen und Forschungsbefunde herangezogen werden.

- „Empirische Untersuchungen“: Die Perspektive von Lehrkräften zu bestimmten Themengebieten sind eine zentrale Einflussgröße für den Unterricht (s. o.) und daher Inhalt der empirischen Aufgaben der Didaktischen Rekonstruktion. In diesem Untersuchungsschritt ist zentral, über welches Wissen über Schülervorstellungen Lehrkräfte verfügen, welche Bedeutung sie fachlichen Unterrichtsinhalten zu einem bestimmten Gegenstandsfeld zugestehen und über welche Ziele und Normen von Unterricht sie verfügen.

- „Didaktische Strukturierung“: Die Ergebnisse der Fachdidaktischen Klärung sowie die Ergebnisse der empirischen Untersuchung der Lehrendenperspektiven werden zur Entwicklung von Elementen für die Lehrerbildung und die Formulierung von Leitlinien aufeinander bezogen. Die konstruktiv erarbeiteten Vorschläge können die Ordnung, Eingrenzung, Durchführung oder auch Entwicklung eines fachlichen Gegenstandsbereiches betreffen.

Abb. 1: Modell der Didaktischen Rekonstruktion für die Lehrerbildung (Komorek/Fischer/ Moschner 2013, S. 47)

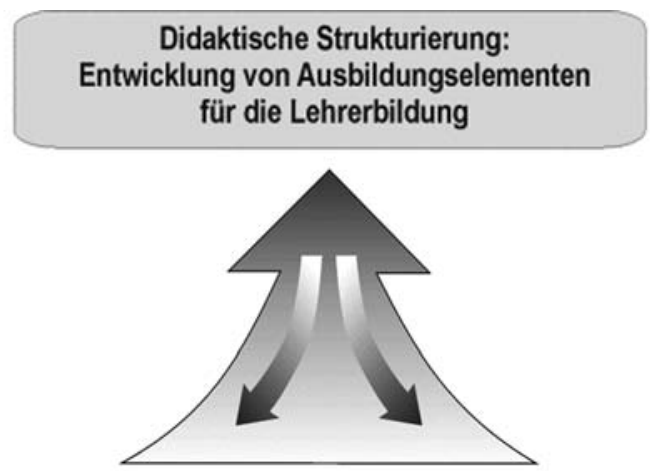

\section{Analyse und fachdidaktische Klärung domänenspezi- fischer fachdidaktischer Konzeptionen}

\section{Empirische Untersuchung subjektiver Überzeugungen von Lehrpersonen zur fach- didaktischen Strukturierung}

Die Untersuchungsaufgaben im Modell der Didaktischen Rekonstruktion erfolgen nicht in stringenter Abfolge, sondern in einem rekursiven Prozess. Die Grundlagen des Modells sind vor diesem Hintergrund anschlussfähig an Kurt Reussers Argumentation zur Zielperspektive design-basierter Forschung: 
Ein wichtiges Merkmal design-basierter Forschung besteht darin, dass Theorieentwicklung und das Kreieren von inhaltsbezogenen Lehr-Lernumgebungen Hand in Hand gehen und sich gegenseitig unterstützen. Davon können die Unterrichtsentwicklung und die Lehrerbildung profitieren. (Reusser 2008, S. 233)

\section{4 ,Sehen' und ,Erkennen` im interdisziplinären Kollektiv: Erkenntnisse aus deutschdidaktischer Perspektive}

In diesem Abschnitt geht es um die Frage, wie die systematische Multiperspektivität in unserem Promotionsprogramm tatsächlich Einfluss auf das eigene deutschdidaktische Forschungsanliegen genommen hat. Die gewählten Beispiele dienen im Weiteren als Grundlage, um zu zeigen,

- wie die Perspektiven anderer Fächer bei der Ausarbeitung eine wichtige Reflexionsfolie für das eigene Vorgehen geboten und den disziplinären Blick erweitert haben.

- wie das interdisziplinäre Arbeiten im Promotionsprogramm befördert hat, vertraute Fachperspektiven im Spiegel anderer Fächer zu hinterfragen und zu differenzieren.

\subsection{Orientierungsrahmen für deutschdidaktische Entwicklungsforschung: Das Modell der Didaktischen Rekonstruktion}

Zum Kerngeschäft einer Deutschdidaktik als praktische Wissenschaft gehört es, „Entscheidungshilfen für die Praxis“"(Ossner 2001, S. 24) bereitzustellen, um zur Verbesserung von Lehr- und Lernprozessen im Deutschunterricht und in der Lehrerbildung beizutragen. Ziel des deutschdidaktischen Promotionsprojekts war es, ein unterrichtsnahes lesediagnostisches Beobachtungsverfahren für die Sekundarstufe I (»JuDiT-L«) zu entwickeln, das Deutschlehrkräfte in ihrer Diagnosepraxis unterstützen soll (vgl. Schmidt 2013). Der Transfer deutschdidaktischer Innovationen und Forschungserkenntnisse in die Schulpraxis erfordert, die Sichtweisen der Lehrkräfte mit zu berücksichtigen: Es gilt, den Horizont der Lehrenden ernst zu nehmen und bereits in den Entwicklungsprozess einzubeziehen, damit fachdidaktische Modellierungen und Impulse tatsächlich handlungsrelevant werden (vgl. u. a. die Ergebnisse von Scherf 2013). Auf Grundlage dieser Überlegungen bildete die Rekonstruktion der Lehrendenperspektiven im Bereich der Diagnose von Lesekompetenz einen zentralen Untersuchungsschritt für die (Weiter-)Entwicklung des unterrichtsnahen Beobachtungsverfahrens (vgl. Schmidt 2013, 261f.). Gleichwohl gibt es in der Fachdidaktik Deutsch bis dato 
keine systematischen Untersuchungen, in denen Bezüge zwischen wissenschaftlichen Konzepten und Lehrendenperspektiven hergestellt werden. Überhaupt sind aktuell in der Deutschdidaktik noch keine ausreichend verlässlichen Prinzipien auszumachen, um eine angemessene Verbindung zwischen beiden Rationalitätsformen herstellen zu können. Kurzum: „Zumindest für die deutschdidaktische Lehrerforschung stellt die reflektierte Relationierung der Wissenssysteme eine nicht unerhebliche Herausforderung dar" (Wieser 2015, S. 21).

Recht erhellend für den deutschdidaktischen Diskurs war insofern das Modell der Didaktischen Rekonstruktion, das ursprünglich aus der Naturwissenschaftsdidaktik stammt (s. o., Abschnitt 3.2). Wie bereits beschrieben, ist das Modell flexibel einsetzbar, um im Kern fachdidaktische Fragestellungen zu bearbeiten. Insofern bestand aus deutschdidaktischer Perspektive die Möglichkeit, an das Modell für das eigene Forschungsanliegen anzuknüpfen, „ohne dabei die eigenen Fragestellungen aufzugeben“ (Reusser 2008, S. 232). Mit dem Modell der Didaktischen Rekonstruktion als Denkrahmen für die eigene fachdidaktische Entwicklungsforschung ergaben sich folgende zentrale Untersuchungsschritte (Abb. 2):

- Untersuchungsaufgabe „Fachdidaktische Klärung“: Analyse der fachlichen und fachdidaktischen Perspektive(n) zum Untersuchungsgegenstand „Diagnose von Lesekompetenz in der Sekundarstufe I“.

- Untersuchungsaufgabe „Empirische Untersuchung von Lehrervorstellungen“: Es wurde weiterhin untersucht, (1) über welche fachlichen Kenntnisse Lehrende im Fach Deutsch zur „Diagnose von Lesekompetenz“ verfügen, (2) welche Kenntnisse sie von Lernendenperspektiven zum Thema haben und (3) welche Kenntnisse sie von verschiedenen Instruktions- bzw. Diagnoseformen zum Gegenstand Lesekompetenz besitzen.

- Untersuchungsaufgabe „Fachdidaktische Strukturierung“: Im Rahmen der Fachdidaktischen Strukturierung wurden die Ergebnisse der analytischen und empirischen Untersuchungsschritte zueinander in Beziehung gesetzt und die Erkenntnisse für die Entwicklung des Diagnoseinstruments » JuDiT-L« produktiv gemacht. 
Abb. 2: Einordnung der Untersuchung in das Modell der Didaktischen Rekonstruktion für die Lehrerbildung

Fachdidaktische Strukturierung

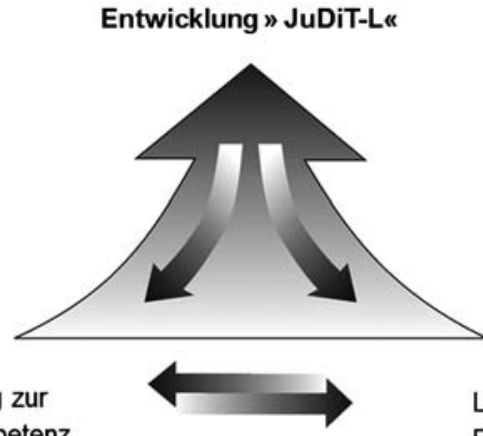

Lehrerperspektiven zur

Fachdidaktische Klärung zur Diagnose von Lesekompetenz

Diagnose von Lesekompetenz

Für die vorliegende Untersuchung bot das Modell der Didaktischen Rekonstruktion einen „belastbaren Orientierungsrahmen“(Komorek/Fischer/Moschner 2013, S. 44) und eine wichtige Reflexionsfolie zur systematischen Konzeption eines praxisnahen Diagnoseinstruments. Hier wird deutlich, wie die (engeren) Grenzen des disziplinären Kerns überschritten wurden und mit Hilfe eines Modells, das sich in anderen Disziplinen als relevant erwiesen hat, das eigene Forschungsfeld konturiert werden konnte.

\subsection{Theoretische Modellierung und Operationalisierung des Konstrukts Vorstellungen}

Inhalt der empirischen Aufgaben in der Didaktischen Rekonstruktion ist die Erhebung und Rekonstruktion von Lehrendenperspektiven (s. o.). Am Anfang des Forschungsprozesses stand daher die normative Frage, wie die zu erhebenden Lehrendenperspektiven zu operationalisieren sind. Zentraler Ansatzpunkt für diesen Untersuchungsschritt war, über welche Wissensbestände zur Diagnose von Lesekompetenz Deutschlehrkräfte verfügen und welche Wissensbestände Niederschlag in ihrem diagnostischen Handeln finden (vgl. Schmidt 2013, S. 263). Im Anschluss an die Systematisierung von Bräuer und Winkler (2012, S. 79) ging es dementsprechend um die Rekonstruktion von lehrerseitigen Wissensbeständen, die „im Labor" und somit verhaltensfern erhoben wurden. Die empirische Lehrerforschung in der Deutschdidaktik kann mittlerweile einige Arbeiten aufweisen, in denen die Rekonstruktion lehrerseitiger Sichtweisen eine zentrale Rolle einnimmt (vgl. ebd., S. 79ff.). Breit rezipiert ist in der Disziplin die Arbeit von 
Dorothee Wieser (2008) zum Lehrerwissen von Deutschreferendaren im Bereich des Literaturunterrichts, die auch für die vorliegende Untersuchung anschlussfähig erschien. Wieser verwendet in ihrer Studie den Begriff „Vorstellungen“, mit dem sie die reflexiven Wissensbestände von Lehrkräften kennzeichnet sowie ergänzend den Begriff „Orientierungen“, um die unhinterfragten Deutungsmuster von Deutschlehrenden in den Blick zu nehmen, welche explizit und implizit vorliegen können (vgl. ebd., S. 60). Weitere Arbeiten in der deutschdidaktischen Lehrerforschung, in denen der Vorstellungsbegriff Anwendung findet, bauen wenn auch mit leichten Modifikationen - auf Wiesers Überlegungen auf (so u. a. Scherf 2013). Eine tiefergehende Konzeptspezifikation fehlt aber sowohl in Wiesers Studie als auch in den weiteren bestehenden Untersuchungen innerhalb der Deutschdidaktik. Vor diesem Hintergrund bot der interdisziplinäre Austausch über die Konkretisierung des Konstrukts „Vorstellungen“ in den Naturwissenschaftsdidaktiken Anstoß, über die bestehenden deutschdidaktischen Setzungen nachzudenken. Breite Anwendung findet in den Naturwissenschaftsdidaktiken die biologiedidaktische Arbeit von Harald Gropengießer (2007). Gropengießer systematisiert Vorstellungen als kognitive gedankliche Konstrukte, die auf unterschiedlichen Komplexitätsebenen beschrieben und erfasst werden können: Von Begriffen, als unterste Komplexitätsebene von Vorstellungen, bis hin zu Theorien als höchste Ebene der Komplexität (vgl. ebd., S. 31ff.). Die unterschiedlichen Komplexitätsstufen von Vorstellungen sind zudem hinsichtlich „des Grades der Bewusstheit" zu unterscheiden (ebd.). Über diese Unterscheidungsdimension ist Gropengießers Konzeptspezifikation anschlussfähig an die bestehende Setzung in der Deutschdidaktik, zwischen impliziten und expliziten Wissensanteilen im Hinblick auf das Lehrerwissen zu unterscheiden (s. o.). Umgekehrt findet in den Konkretisierungen der Naturwissenschaftsdidaktik die soziale Bedingtheit des Lehrerwissens keine Berücksichtigung. In Anlehnung an die Studie von Wieser wurde daher festgelegt, zwischen individuellen und sozial geteilten Wissensbeständen zur Erhebung der Lehrendenperspektiven zu unterscheiden. Auf dieser Grundlage wurden im Forschungsprojekt sowohl Vorstellungen als subjektive gedankliche Konstrukte, die sich im Grad der Komplexität und im Grad der Bewusstheit unterscheiden, als auch die Orientierungen als die unhinterfragten, sozial geteilten Deutungsmuster der Deutschlehrkräfte rekonstruiert, um ein unterrichtsnahes Diagnoseinstrument zu entwickeln (vgl. Schmidt 2015, S. 91f.). Dieses Beispiel zeigt, wie Konstrukte, die sich in anderen Fächern als relevant erwiesen haben, Impulse bieten können, um bestehende Setzungen in der eigenen Disziplin vor einem neuen Hintergrund zu reflektieren. 


\section{Interdisziplinäres Denken und Handeln, Lehren und Lernen - Erkenntnisse aus ProfaS}

Konkrete Aspekte interdisziplinärer Arbeit entstehen erst im Forschungsprozess. Daher wollen wir abschließend einige Erkenntnisse und Schlussfolgerungen aus der gemeinsamen Arbeit im Promotionsprogramm ProfaS erörtern. Unsere Reflexion erfolgt dabei auf zwei Ebenen: Zum einen in Hinblick auf unseren fächerspezifischen Zugang und zum anderen hinsichtlich unserer unterschiedlichen Positionen im Programm als Doktorandin bzw. als Betreuerin.

\subsection{Interdisziplinär promovieren - Perspektive der Deutschdidaktikerin/Doktorandin}

(1) Von selbstverständlichen Prämissen zur Klarheit und Transparenz von Begriffen, Modellen und Erkenntnissen im Forschungsprojekt

In Abschnitt 2.2 haben wir den grounding-Prozess als zentrales Element für die interdisziplinäre Verständigung im Promotionsprogramm beleuchtet. Für mich sorgte diese „Arbeit des Aufdeckens und Diskutierbarmachens“ (Prediger 2003, S. 12) im Hinblick auf die Ausarbeitung meines eigenen Promotionsprojekts für Klarheit und Fokussierung in Bezug auf Begriffe, Überzeugungen und Herangehensweisen. Die interdisziplinäre Verständigung über meine Forschungsarbeit war für mich immer wieder mit dem positiven ,Zwang' verknüpft, vertraute deutschdidaktische Begriffe, Konstrukte oder Modelle zu explizieren bzw. zu klären, was mitunter weniger notwendig sein dürfte, wenn man mit Vertretern des eigenen Denkkollektivs zusammenkommt (s. o., Abschnitt 2.2). So kann ich Klaus Immelmann zustimmen, wenn er festhält, dass der interdisziplinäre Dialog

immer dann zu neuen Einsichten und gegenseitigen Brückenschlägen geführt [hat], wenn Teilnehmer der einen Disziplin gerade in ihren sichersten Annahmen durch einen Vertreter der anderen Disziplin verunsichert wurden [...]. (Immelmann 1987, S. 89)

Gerade diese Irritationsmomente führten in der Rückschau auch immer wieder dazu, dass für mich die eigene Forschung als Prozess deutlich erkennbar wurde.

(2) Von impliziten Normen zum Wahrnehmen und Einordnen des eigenen Denkstils Durch die mehrjährige Auseinandersetzung mit Modellen, Theorien und Erkenntnissen benachbarter Disziplinen wurde ich immer wieder dazu angeregt, einen reflexiven Blick auf Setzungen der Deutschdidaktik einzunehmen. Gerade die Auseinandersetzung mit den Denkstilen andere Fächer im Rahmen des gleichen Themengebiets, also der Lehrerforschung, hat aus meiner Perspektive dazu 
beigetragen, dass das eigene Fach klar(er) profiliert wurde. Die interdisziplinäre Arbeit bot Ansätze zur „Selbstkontrolle und Selbstreflexion“ (Kocka 1987, S. 10) im Hinblick auf mein Selbstverständnis als Deutschdidaktikerin, für die folgende Fragestellungen kennzeichnend sind: Was hat das, was ich hier anders erlebe, mit meiner eigenen Disziplin zu tun? Wie werden Forschungswege und methodische Zugänge in der Deutschdidaktik begriffen? Was erzeugt für mich Irritationen und warum? Welche Modelle und Konstrukte anderer Fächer möchte ich übernehmen und/oder inwiefern muss ich diese noch fachspezifisch profilieren? Diese Fragen verdeutlichen, wie im Rahmen des Programms ein Modus des Denkens ausgebildet wurde, der im Kern „die Außenlinien und damit das innere Feld“ (Ossner 2001, S. 29), also den Denkrahmen der Deutschdidaktik berührt, da er immer wieder gezielt zum Reflexionsgegenstand wurde. ${ }^{10}$

(3) Vom interdisziplinären Dialog zur Erkenntnisgewinnung in der eigenen Disziplin Trotz der Möglichkeiten zum produktiven Dialog zwischen den Fachkulturen, die ich bislang diskutiert habe, bleibt aber bestehen, dass die Kernkompetenzen des eigenen Faches in der interdisziplinären Arbeit bewahrt werden müssen (z. B. um Konstrukte fachspezifisch zu profilieren). Zudem sind die Ergebnisse meines eigenen Forschungsprojekts erst im Horizont des eigenen Faches verständlich geworden wie auch die Anwendung der Erkenntnisse meiner Arbeit nur in der Deutschdidaktik selbst erfolgen kann (vgl. zu diesem Aspekt allgemein Kocka 1987, S. 9). Insofern kann man im Anschluss an Heckhausen festhalten, dass „Forschung im Sinne schärfster Erkenntnis nur intradisziplinär [...] betrieben werden kann“ (Heckhausen 1987, S. 139).

\subsection{Interdisziplinär Lehren - Erkennen und Kommunizieren aus Perspektive der Lehr-Lernforscherin/Betreuerin}

Auch für die Betreuerinnen und Betreuer der Arbeiten ist die Zusammenarbeit in einem interdisziplinären Promotionsprogramm ein Kontext mit hohem Lernpotential. Diese Möglichkeit wird unterschiedlich genutzt, deshalb sollen an dieser Stelle die eigenen Erfahrungen zentral sein.

\section{(1) Inhaltliche Perspektiven}

In einem Promotionsprogramm, in dem fachdidaktische Professorinnen und Professoren deutlich zahlreicher vertreten sind als pädagogische und psychologische

10 Vgl. zur Bedeutung des Reflexionsansatzes für das eigene Lernen Schön (1983) und Neuweg (2011). Siehe dazu auch den Beitrag von Winkler et al., in diesem Band. 
Professorinnen und Professoren, wurde zunächst immer wieder die Kritik von Dietrich (1994, S. 235): „Allgemeindidaktik ist wie Stricken ohne Wolle“ und später von Endres (2007) „Lernen lernen ist wie Stricken ohne Wolle“ geäußert. Bei genauerem Hinsehen zeigte sich jedoch, dass wesentliche Inhalte, Modelle und Theorien aus den Disziplinen der Pädagogik und Psychologie in der fachdidaktischen Forschung (zum Teil lediglich sehr leicht modifiziert, zum Teil unverändert) aufgegriffen und beforscht werden. Das Programm hat also dazu beigetragen, solche unproduktiven Konfrontationen aufzulösen.

\section{(2) Methodische Perspektiven}

$\mathrm{Zu}$ Beginn der gemeinsamen Promotionsprogramme (im Jahr 2001) waren die methodischen Herangehensweisen an die Forschungsfragen sehr disparat. Während mein eigener methodischer Hintergrund sehr quantitativ geprägt war (Auswertung von großen Datensätzen mit quantitativen Verfahren), wurde in den Fachdidaktiken fast ausschließlich ein qualitativer Ansatz (mit sehr kleinen Fallzahlen) präferiert. Die kontinuierliche Auseinandersetzung mit anderen methodischen Zugangsweisen und Settings hat dazu beigetragen, die jeweils andere Zugangsweise besser zu verstehen und wertzuschätzen. Zudem wurde eine Vielzahl neuer Verfahren erprobt (vgl. Kaiser 2015). Nicht zuletzt die zunehmende Forderung nach „Mixed-Method-Ansätzen“ (Kuckartz 2014) kann durch die verschiedenen vorhandenen Kompetenzen im Kollegium nun besser umgesetzt werden.

\section{(3) Anwendungsbezogene Forschung}

Neu - und gewöhnungsbedürftig - war für mich auch der unbedingte Wille, Forschung für die Praxis zu machen. In meiner eigenen Disziplin - der Psychologie - gilt Grundlagenforschung als die Krone der Forschung. Als etwas weniger wertvoll wurde in der Psychologie Grundlagenforschung betrachtet, die auf Praxisprobleme übertragen werden konnte. Mit anwendungsbezogener Forschung konnte man sich ,die Finger schmutzig' machen. Ganz anders der Ansatz in den Fachdidaktiken. Hier gilt die anwendungsbezogene Forschung als die einzig akzeptable Forschung schlechthin. Forschung ohne Praxisbezug wird als sinnlos angesehen. Ein gemeinsames Miteinander in diesem Spannungsfeld erfordert Toleranz von allen Seiten. Aus meiner Sicht ist dies in ProfaS sehr gut gelungen. Auch wenn das Ziel „Forschung für die Praxis“ durch die Vorgabe „Fachdidaktische Strukturierung“ (s. Abb.1) als eindeutige Erwartung formuliert ist (und auch von allen akzeptiert wurde), zeigten sich doch bei der praktischen Umsetzung dieser Vorgabe immer wieder Probleme. Es wurde deutlich, dass sich nicht jedes Forschungsergebnis einfach in eine Praxisempfehlung umsetzen lässt. Dass 
immer auch die Perspektive der Lernenden berücksichtigt werden muss, ist in diesem Kontext von größter Bedeutung.

(4) Das Ganze ist mehr als die Summe seiner Teile - mit Toleranz und gegenseitiger Achtung gemeinsam etwas Neues schaffen

Die Zusammenarbeit in einem relativ großen Promotionsprogramm mit vielen Akteuren kann nur gelingen, wenn die Beteiligten bereit sind, eigene Gewohnheiten infrage zu stellen, sich von der Bedeutung der eigenen Disziplin zu distanzieren, sich in andere Disziplinen einzuarbeiten und sich in die Rolle des Lernenden zu begeben. Zudem gilt es, die Potentiale der eigenen Disziplin für andere fruchtbar zu machen. Erst durch ein gegenseitiges Geben und Nehmen kann eine multiperspektivische Sichtweise eingenommen werden, die über das hinausgeht, was in den einzelnen Disziplinen die ,typische 'Herangehensweise an Forschung ist. Dies ist im Promotionsprogramm ProfaS nicht nur durch einen wertschätzenden Umgang der Lehrenden untereinander, sondern auch durch eine hohe Wertschätzung und Achtung der Promovierenden und deren Ideen, methodischen Herangehensweisen und disziplinübergreifenden Kooperationen gelungen. „Der uneingeschränkte Wille zu lernen und die Bereitschaft, die eigenen disziplinären Vorstellungen zur Disposition zu stellen“ (Mittelstraß 2012, S. 11) hat allen Beteiligten geholfen, über sich selbst hinauszuwachsen.

\section{Bilanzierende Bemerkungen}

Interdisziplinarität an sich hat keinen Eigenwert. Wenn verschiedene Disziplinen an einem gemeinsamen Ziel bzw. einer Problemstellung arbeiten, so unsere Beobachtung, stellt dies nicht bereits automatisch forschungsbezogene Dynamik und schon gar nicht Erkenntnisgewinn und neues Wissen her. ${ }^{11}$ Die gemeinsame Arbeit im Rahmen von ProfaS hat für uns verdeutlicht, dass tatsächliche interdisziplinäre Zusammenarbeit vielmehr etwas aktiv Geschaffenes und nichts von vornherein Bestehendes ist - sie ist das Ergebnis eines Prozesses. Gleichfalls werden die von uns dargelegten (inter-)disziplinären Kompetenzen nicht einfach durch das Erfahren und die Teilnahme an einem fächerübergreifenden Promotionsprogramm

11 Vgl. vertiefend zu diesem Gedankengang die Ausführungen von Susanne Prediger, die sehr treffend die Parallelisierung der Diskussionen über interdisziplinäre Zusammenarbeit und die sog. „Kulturkontakt-Hypothese“ erläutert und darauf aufbauend bezweifelt, dass "die bloße Begegnung der [wissenschaftlichen] Kulturen schon Lernprozesse auslöst" (Prediger 2003, S. 8). 
erworben. Langfristig waren die aktive Beteiligung und Offenheit des Einzelnen wichtige Voraussetzungen, um entsprechende Kompetenzen zu erwerben. ${ }^{12}$

Trotz des Gewinns, den wir in der gemeinsamen Arbeit in ProfaS gesehen haben, war und ist die eigene Disziplin der zentrale Bezugspunkt für Promovierende und Betreuende gewesen. Dieser Aspekt kommt spätestens dann zum Tragen, wenn es um die Frage(n) der Implikationen aus den eigenen Forschungsbefunden geht. Prinzipiell müssen Forschungsprojekte und Qualifikationsarbeiten in der eigenen scientific community sichtbar und fortgesetzt werden - nicht zuletzt, da sie über das Ansehen von Forschungsprojekten und das eigene berufliche Fortkommen entscheidet. Darüber hinaus, und das haben wir an verschiedenen Stellen betont, ist die Rückbindung an die eigene Fachdisziplin zentral, um erfolgreich interdisziplinär arbeiten zu können: die Disziplinen sind die „Garantinnen der Interdisziplinarität“" (Defila/Di Giulio 1998, S. 125).

Eine wesentliche Erfolgsbedingung für unser Promotionsprogramm war, dass sich die beteiligten Disziplinen, Betreuer wie Doktoranden, in einer selbstbestimmten Bedeutungsebene als kritische Freundinnen und nicht als „fremde Schwestern“ (Terhart 2002) gesehen haben. Basierend auf dieser „Vertrauenskultur" (Frey 2000, S. 81) konnte man vom Erfahrungs- und Forschungswissen untereinander nur profitieren. Bereits Fleck bündelt diese Erfahrungswerte, wenn er argumentiert:

[...] Denkstilveränderung - d. h. Veränderung der Bereitschaft für gerichtetes Wahrnehmen - gibt neue Entdeckungsmöglichkeiten und schafft neue Tatsachen. Dies ist die wichtigste erkenntnistheoretische Bedeutung des interkollektiven Denkverkehrs. (Fleck 1935, S. 144)

\section{Literatur}

Baumert, Jürgen/Kunter, Mareike (2006): Stichwort: Professionelle Kompetenz von Lehrkräften. In: Zeitschrift für Erziehungswissenschaft. Jg. 9, H. 4, S. 469-520.

Bräuer, Christoph/Winkler, Iris (2012): Aktuelle Forschung zu Deutschlehrkräften. Ein Überblick. In: Didaktik Deutsch. H. 33, S. 74-91.

Bräuer, Christoph/Wieser, Dorothee (Hrsg.) (2015): Lehrende im Blick. Empirische Lehrerforschung in der Deutschdidaktik. Wiesbaden: Springer VS.

12 So war auch in unserem Programm zu beobachten, dass die einzelnen Beteiligten ihre Rolle unterschiedlich wahrgenommen haben. Vgl. zu diesem Aspekt allgemein Defila/ Di Giulio (1998, S. 123ff.). 
Bromme, Rainer (1999): Die eigene und die fremde Perspektive: Zur Psychologie kognitiver Interdisziplinarität. In: Umstätter, Walter/Wessel, Karl-Friedrich (Hrsg.): Interdisziplinarität - Herausforderung an die Wissenschaftlerinnen und Wissenschaftler. Festschrift zum 60. Geburtstag von Heinrich Parthey. Bielefeld: Kleine, S. 37-61.

Bromme, Rainer/Jucks, Regina/Rambow, Riklef (2004): Experten-Laien-Kommunikation im Wissensmanagement. In: Reinmann, Gabi/Mandl, Heinz (Hrsg.): Psychologie des Wissensmanagements. Perspektiven, Theorien und Methoden. Göttingen u. a.: Hogrefe, S. 176-188.

Defila, Rico/Di Giulio, Antonietta (1998): Interdisziplinarität und Disziplinarität. In: Obertz, Jan H. (Hrsg.): Zwischen den Fächern über den Dingen? Universalisierung versus Spezialisierung akademischer Bildung. Opladen: Leske \& Budrich, S. 111-137.

Dietrich, Ingrid (1994): „Allgemeine Didaktik ist wie Stricken ohne Wolle“ - Zur Bedeutsamkeit des Streits der Disziplinen. In: Meyer, Meinhard/Plöger, Wilfried (Hrsg.): Allgemeine Didaktik, Fachdidaktik und Fachunterricht. Weinheim: Beltz, S. 235-242.

Endres, Wolfgang (Hrsg.) (2007): Lernen lernen - Wie stricken ohne Wolle. Beltz: Weinheim.

Fleck, Ludwik (1994) [1935]: Entstehung und Entwicklung einer wissenschaftlichen Tatsache. Einführung in die Lehre vom Denkstil und Denkkollektiv. Mit einer Einleitung herausgegeben von Lothar Schäfer und Thomas Schnelle. 3. Aufl. Frankfurt a. M.: Suhrkamp.

Fleck, Ludwik (2011) [1936]: Das Problem einer Theorie des Erkennens. In: Ludwik Fleck. Denkstile und Tatsachen. Gesammelte Schriften und Zeugnisse. Herausgegeben von Sylwia Werner und Claus Zittel. Berlin: Suhrkamp, S. 260-309.

Frey, Dieter (2000): Kommunikations- und Kooperationskultur aus sozialpsychologischer Sicht. In: Mandl, Heinz/Reinmann-Rothmeier, Gabi (Hrsg.): Wissensmanagement. Informationszuwachs - Wissensschwund? Die strategische Bedeutung des Wissensmanagements. München/Wien: Oldenbourg, S. 73-92.

Gropengießer, Harald (2007): Didaktische Rekonstruktion des "Sehens". Wissenschaftliche Theorien und die Sicht der Schüler in der Perspektive der Vermittlung. Nachdr. der 2., überarb. Aufl. Oldenburg: Zentrum für Pädagogische Berufspraxis.

Heckhausen, Heinz (1987): „Interdisziplinäre Forschung“ zwischen Intra-, Multi- und Chimären-Disziplinarität. In: Kocka, Jürgen (Hrsg.): Interdisziplinarität. Praxis - Herausforderungen - Ideologie. Frankfurt a. M.: Suhrkamp, S. $129-145$. 
Hofstein, Avi/Carmi, Miriam/Ben-Zvi, Ruth (2003): The Development of Leadership among Chemistry Teachers in Israel. In: International Journal of Science and Mathematics Education. Vol. 1. No. 1, pp. 39-65.

Immelmann, Klaus (1987): Interdisziplinäre Wissenschaftspraxis. Erfahrungen und Kriterien. In: Kocka, Jürgen (Hrsg.): Interdisziplinarität. Praxis - Herausforderungen - Ideologie. Frankfurt a. M.: Suhrkamp, S. 82-91.

Kaiser, Astrid (Hrsg.) (2015): Innovative Erhebungsmethoden - dargestellt an verschiedenen Forschungsprojekten. Baltmannsweiler: Schneider Hohengehren.

Kattmann, Ulrich (2007): Didaktische Rekonstruktion - eine praktische Theorie. In: Krüger, Dirk/Vogt, Helmut (Hrsg.): Theorien in der biologiedidaktischen Forschung. Ein Handbuch für Lehramtsstudenten und Doktoranden. Berlin/ Heidelberg: Springer, S. 93-104.

Kattmann, Ulrich/Duit, Reinders/Gropengießer, Harald/Komorek, Michael (1997): Das Modell der Didaktischen Rekonstruktion - Ein Rahmen für naturwissenschaftsdidaktische Forschung und Entwicklung. In: Zeitschrift für Didaktik der Naturwissenschaften. Jg. 3. H. 3, S. 3-18.

Kocka, Jürgen (1987): Einleitung. In: Kocka, Jürgen (Hrsg.): Interdisziplinarität. Praxis - Herausforderungen - Ideologie. Frankfurt a. M.: Suhrkamp, S. 7-16.

Komorek, Michael/Fischer, Astrid/Moschner, Barbara (2013): Fachdidaktische Strukturierung als Grundlage für Unterrichtsdesigns. In: Komorek, Michael/ Prediger, Susanne (Hrsg.): Der lange Weg zum Unterrichtsdesign - Zur Begründung und Umsetzung genuin fachdidaktischer Forschungs- und Entwicklungsprogramme. Münster: Waxmann, S. 37-57.

Kuckartz, Udo (2014): Mixed Methods. Methodologie, Forschungsdesigns und Analyseverfahren. Wiesbaden: Springer.

Mittelstraß, Jürgen (2012): Transdisziplinarität oder: von der schwachen zur starken Interdisziplinarität. In: Gegenworte. Hefte für den Disput über Wissen. H. 28, S. 10-13.

Neuweg, Hans Georg (2011): Das Wissen der Wissensvermittler - Problemstellungen, Befunde und Perspektiven der Forschung zum Lehrerwissen. In: Terhart, Ewald/Bennewitz, Hedda/Rothland, Martin (Hrsg.): Handbuch der Forschung zum Lehrerberuf. Münster: Waxmann, S. 451-477.

Ossner, Jakob (2001): Elemente eines Denkstils für didaktische Entscheidungen. In: Rosebrock, Cornelia/Fix, Martin (Hrsg.): Tumulte. Deutschdidaktik zwischen den Stühlen. Baltmannsweiler: Schneider Hohengehren, S. 17-32.

Prediger, Susanne (2003): Universitäre Wissenskultur: Einheit der Wissenschaften in der multikulturellen Vielfalt der Disziplinen. In: Lengnink, Katja/Prediger, Susanne/Siebel, Franziska (Hrsg.): Mathematik für Menschen. Festschrift für Rudolf Wille zur Emeritierung. Darmstadt: Eigendruck. Zitiert nach: 
http://www.mathematik.uni-dortmund.de/ prediger/veroeff/03-festschriftfachkulturen.pdf. Abgerufen am 28.09.2015.

Reusser, Kurt (2008): Empirisch fundierte Didaktik - didaktisch fundierte Unterrichtsforschung. In: Meyer, Meinert/Prenzel, Michael/Hellekamps, Stephanie (Hrsg.): Perspektiven der Didaktik. Zeitschrift für Erziehungswissenschaft. Sonderheft 9, S. 219-237.

Scherf, Daniel (2013): Leseförderung aus Lehrersicht. Eine qualitativ-empirische Untersuchung professionellen Wissens. Wiesbaden: Springer VS.

Schmidt, Frederike (2013): Den Schüler im Blick - Zur Entwicklung und Erprobung eines Diagnosetools für den Bereich Lesekompetenz. In: Komorek, Michael/Prediger, Susanne (Hrsg.): Der lange Weg zum Unterrichtsdesign Zur Begründung und Umsetzung genuin fachdidaktischer Forschungs- und Entwicklungsprogramme. Münster u. a.: Waxmann, S. 257-272.

Schmidt, Frederike (2015): Den diagnostischen Blick schärfen: Vorstellungen und Orientierungen von Deutschlehrerinnen und Deutschlehrern zur Diagnose von Lesekompetenz. In: Bräuer, Christoph/Wieser, Dorothee (Hrsg.): Lehrende im Blick. Empirische Lehrerforschung in der Deutschdidaktik. Wiesbaden: Springer VS, S. 87-107.

Schön, Donald A. (1983): The Reflective Practitioner. How Professionals Think in Action. London: Temple Smith.

Shulman, Lee S. (1986): Those who understand: Knowledge growth in teaching. In: Educational Researcher. Vol. 15. No. 2, pp. 4-14.

Sukopp, Thomas (2010): Interdisziplinarität und Transdisziplinarität. Definitionen und Konzepte. In: Jungert, Michael/Romfeld, Elsa/Sukopp, Thomas/Voigt, Uwe (Hrsg.): Interdisziplinarität. Theorie, Praxis, Probleme. Darmstadt: Wissenschaftliche Buchgesellschaft, S. 13-29.

Terhart, Ewald (2002): Fremde Schwestern. Zum Verhältnis von Allgemeiner Didaktik und empirischer Lehr-Lern-Forschung. In: Zeitschrift für Pädagogische Psychologie. Jg. 16. H. 2, S. 77-86.

Terhart, Ewald (2011): Zur Situation der Fachdidaktiken aus der Sicht der Erziehungswissenschaft. Konzeptionelle Probleme, institutionelle Bedingungen, notwendige Perspektiven. In: Bayrhuber, Horst/Harms, Ute/Muszynski, Bernhard/Ralle, Bernd/Rothgangel, Martin/Schön, Lutz-Helmut/Vollmer, Helmut Johannes/Weigand, Hans-Georg (Hrsg.): Empirische Fundierung in den Fachdidaktiken. Münster u. a.: Waxmann, S. 241-256.

Terhart, Ewald/Bennewitz, Hedda/Rothland, Martin (Hrsg.) (2014): Handbuch der Forschung zum Lehrerberuf. Münster/New York: Waxmann. 
Van Dijk, Esther M./Kattmann, Ulrich (2007): A research model for the study of science teachers' PCK and improving teacher education. In: Teaching and Teacher Education. No. 23, pp. 885-897.

Wieser, Dorothee (2008): Literaturunterricht aus Sicht der Lehrenden. Eine qualitative Interviewstudie. Wiesbaden: Springer.

Wieser, Dorothee (2015): Theorie(?)-Praxis-Konstellationen in Lehrerforschung und Lehrerbildung: Fragen an die aktuelle deutschdidaktische Lehrerforschung. In: Bräuer, Christoph/Wieser, Dorothee (Hrsg.): Lehrende im Blick. Empirische Lehrerforschung in der Deutschdidaktik. Wiesbaden: Springer VS, S. 17-34.

Winkler, Iris (2015): „Subjektive Involviertheit und genaue Wahrnehmung miteinander ins Spiel bringen“. Überlegungen zur Spezifikation eines zentralen Konzepts für den Literaturunterricht. In: Leseräume. H. 2, S. 155-168. 
Iris Winkler and Frederike Schmidt - 978-3-631-69285-1

Downloaded from PubFactory at 01/11/2019 10:32:53AM

via free access 
Thomas Lindauer, Claudia Schmellentin, Anne Beerenwinkel

\title{
Sprachbewusster Naturwissenschafts- Unterricht - Werkstattbericht zu einem transdisziplinären Entwicklungsprojekt
}

\begin{abstract}
How to conceptualize science teaching with a special focus on language aspects was the topic of an interdisciplinary and transdisciplinary Swiss research project. Results of this development project are presented along with some general reflections on how to teach different subjects in a language-sensitive way. Consequences with regard to a collaboration between Language Education and other areas such as Science Education are discussed.
\end{abstract}

Der folgende Beitrag präsentiert Konzeption und Ergebnisse eines inter- bzw. in Ansätzen transdisziplinären Entwicklungsprojekts, das durch die Bildungsdirektionen von vier Schweizer Kantonen (sog. Bildungsraum Nordwestschweiz) mitinitiiert und finanziert wurde. ${ }^{1}$ An diesem Beispiel soll das Potenzial der transdisziplinären Arbeit im Sinne von Stock/Burton (2011) für fachdidaktische Entwicklungsprojekte aufgezeigt und diskutiert werden.

Im ersten Abschnitt skizzieren wir die Konzeption kooperativer Forschungsprojekte und klären dabei die Begriffe Inter- und Transdisziplinarität. In Abschnitt 2 stellen wir dann einige grundsätzliche Überlegungen zur Konzeption eines sprachbewussten Fachunterrichts und der daraus resultierenden Konsequenz einer engen interdisziplinären Zusammenarbeit zwischen Sprach- und anderen Fachdidaktiken dar. Das inter- und transdisziplinäre Entwicklungsprojekt selbst, das einen sprachbewussten Fachunterricht etablieren will, wird dann in Abschnitt 3 dargestellt. ${ }^{2}$

1 Gedankt sei an dieser Stelle Regina Kuratle, die das Projekt wesentlich mitinitiiert hat, sowie Marie-Therese Schönbächler, die das Projekt als Leiterin der interkantonalen Steuergruppe begleitet hat. Zudem gilt unser Dank Julienne Furger und Claudia Hefti, die im Projekt auf Seiten der Deutschdidaktik mitgearbeitet und wesentlich zum Gelingen des Projekts beigetragen haben.

2 Am Projekt beteiligt waren interdisziplinär arbeitende Sprach- und Naturwissenschaftsdidaktikerinnen und -didaktiker sowie transdisziplinär eng zusammenarbeitend eine kleine Steuerungsgruppe aus der Bildungsplanung sowie weniger eng mit dem Projekt verbunden eine größere bildungspolitische Echogruppe und mitberatende Lehrpersonen der Klassen 5-9. 


\section{Inter- und transdisziplinäre Projekte}

Mit dem Begriff „Interdisziplinarität“ werden unterschiedliche Ausprägungen von kooperativen bzw. integrativen Forschungs- und Entwicklungsprojekten verstanden. Stock und Burton (2011) folgend möchten wir hier zwischen Inter- und Transdisziplinariät unterscheiden. ${ }^{3}$ Mit diesen beiden Begriffen sind unterschiedliche Verortungen, Gelingensbedingungen sowie Organisationsstrukturen für Forschungs- und Entwicklungsprojekte verbunden, die als Folie für das nachfolgend dargestellte Entwicklungsprojekt genutzt werden sollen.

Auf den ersten Blick scheinen Fachdidaktiken grundsätzlich ,inhärent interdisziplinär' ${ }^{4}$ zu sein, denn Fachdidaktiken verknüpfen Wissensbestände und theoretische Konzepte aus unterschiedlichen (akademischen) Disziplinen. So schreibt Wittmann (1995, S. 329):

Wie jede andere Fachdidaktik kann auch die Mathematikdidaktik nur in interdisziplinärer Grenzüberschreitung betrieben werden, indem sie auf die Ergebnisse und Methoden der Mathematik, der Allgemeinen Didaktik, der Pädagogik und Psychologie zurückgreift.

3 Stock und Burton (2011) haben ihre Konzeption, die unter anderem auch auf der medizinwissenschaftlichen Literaturstudie von Aboelela et al. (2007) basiert, vor allem mit Blick auf Forschungsprojekte zur Nachhaltigkeit (Sustainability) entwickelt. Die Terminologie wird aber auch mit Gewinn von anderen integrated research approaches etwa aus den Ingenieurswissenschaften adaptiert (vgl. Adame 2011; Carbone/EkwaroOsire 2010). Gerade in Hinblick auf fachdidaktische Entwicklung scheint uns dies ein fruchtbarer Klassifizierungsversuch zu sein, da damit nicht nur eine Begrifflichkeit etabliert wird, sondern weil damit auch konzeptionelle Unterschiede zwischen fachdidaktischen Forschungs- und Entwicklungsprojekten deutlicher werden: Entwicklungsprojekte erfordern meist mehrere Kooperationspartner, insbesondere auch solche außerhalb von fachdidaktischen und pädagogischen Disziplinen (vgl. dazu auch die Beiträge in Bogner/Kastenhofer/Torgensen 2010).

4 In Ermangelung eines präziseren Begriffs dafür, dass Fachdidaktiken Wissensbestände und Forschungsmethoden anderer Disziplinen bedienen, ohne dass man dabei von Interdisziplinarität im eigentlichen Sinn sprechen kann, verwenden wir hier die Formulierung ,inhärent interdisziplinär': Wollen sich Fachdidaktiken als eigenständige akademische Disziplinen verstehen, kann eine Fachdidaktik nicht in sich selbst interdisziplinär sein, sondern erst als eigenständige Disziplin kann sie inter-disziplinär mit anderen Disziplinen zusammenarbeiten. Aufgrund ihrer ,inhärenten Interdisziplinarität' scheinen uns Fachdidaktiken - insbesondere auch untereinander - dafür prädestiniert zu sein, interdisziplinär zu arbeiten. 
Durch spezifisch fachdidaktische Fragestellungen und eine sich daraus entwickelnde zunehmend spezifische fachdidaktische Forschung versuchen sich Fachdidaktiken als eigenständige (akademische) (Forschungs-)Disziplinen zu etablieren:

Wissenschaftliche Erkenntnisse über das Lernen und Lehren von Mathematik sind jedoch nicht als mixtum compositum dieser Wissenschaften zu gewinnen. Es bedarf vielmehr einer spezifischen mathematikdidaktischen Forschung. (Wittmann 1995, S. 330)

Sie sind in dem Sinn, inhärent interdisziplinär.' Interdisziplinäre Projekte im engeren Sinn erfordern das

Zusammenwirken von Personen aus mindestens zwei [Forschungs-]Disziplinen im Hinblick auf gemeinsame Ziele, in welchem die disziplinären Sichtweisen zu einer Gesamtsicht zusammengeführt werden. Einbezogen werden jeweils diejenigen Disziplinen, die zur Bearbeitung des Themas etwas beitragen können. (Defila/Di Giulio 1999, S. 6)

Diese enge Fassung des Begriffs , Interdisziplinarität, ${ }^{5}$ welche für die theoretische Fundierung hilfreich und funktional angemessen ist, eröffnet für Entwicklungsprojekte - wie das im Folgenden dargestellte - ein definitorisches, aber auch konzeptuelles Problem: In Entwicklungsprojekten sind nicht nur Wissensbestände aus disziplinär verorteten Forschungen relevant, sondern ebenfalls Wissensbestände aus dem Praxisfeld, das heißt, Akteure, die nicht in einer Forschungsdisziplin zu verorten sind. Im Kontext von Projekten aus der Bildungsforschung sind damit nicht nur Lehrpersonen und Lernende gemeint, sondern auch Schulleitungen, Akteure aus der (staatlichen) Bildungsplanung, der Curriculumsentwicklung bis hin zu Akteuren aus Lehrmittelverlagen.

Um auch diese Form der Interdisziplinarität begrifflich zu fassen, hat sich der Begriff der Transdisziplinarität etabliert:

„Transdisziplinarität" wiederum wird verstanden als eine spezielle Form von Interdisziplinarität, in der auch die ausserwissenschaftliche Praxis an den Forschungsarbeiten beteiligt wird. Zugrunde gelegt wird eine problemorientierte Forschung, eine Forschung also, die einen Beitrag leisten will zur Lösung (und Vermeidung) gesellschaftlich relevanter Probleme. (Defila/Di Giulio 1999, S. 6)

5 Von interdisziplinären Projekten sind multidisziplinäre zu unterscheiden, auf die wir im Folgenden jedoch nicht näher eingehen werden: Als multidisziplinäre Projekte gelten Projekte, in denen die Disziplinen sich aus je eigener Perspektive, mit eigenen Theorien und Forschungsmethoden dem Problemfeld annehmen. Es besteht ein eher loser Austausch zwischen den verschiedenen Disziplinen, die Ergebnisse werden kaum zusammen publiziert, sondern je in der eigenen wissenschaftlichen Community (vgl. Aboelela et al. 2007; Klein Thompsen 2010). 
Autoren wie Stock/Burton (2011) gehen hier noch einen Schritt weiter: Unter Transdisziplinarität verstehen sie nicht nur die Ausweitung der beteiligten Gruppen über die akademischen Disziplinen hinaus, sondern insbesondere auch die Etablierung eines Disziplinen- bzw. Akteursgruppen-übergreifenden Zugangs:

In this sense transdisciplinarity is the highest form of integrated project, involving not only multiple disciplines, but also multiple non-academic participants (e.g., land managers, user groups, the general public) in a manner that combines interdisciplinarity with participatory approaches. (Stock/Burton 2011, S. 1098)

Eine solche transdisziplinäre Zusammenarbeit scheint uns im Kontext von Entwicklungsprojekten für die Schule und beim Thema des hier vorgestellten Projekts - sprachbewusster Fachunterricht - von besonderer Relevanz zu sein: Für die Etablierung eines sprachbewussten Fachunterrichts genügt es nicht, wenn aus der Deutschdidaktik bspw. Konzepte für das Lesen von Sachtexten in einen Fachunterricht transportiert werden - dafür sind die jeweiligen Fachsprachen und -traditionen, aber auch die jeweiligen fachspezifischen Textformen zu different (vgl. u. a. Schleppegrell 2004; Schmellentin et al. 2011; Shanahan et al. 2011). Erst durch eine gemeinsame Konzeptentwicklung für fachadäquate Lesestrategien oder für fachsprachlich angemessene Textvereinfachung und erst durch eine so entstehende gemeinsame Sprache über Lernstrategien etc. kann sich ein sprachbewusster Fachunterricht in der Schule etablieren.

Transdisciplinarity requires the greatest synthesis of approach. In this mode, teams not only share a common question but also often share and borrow methods, create a common conceptual framework, and either learn each other's disciplinary language or create a new common language. (Aboelela et al. 2007, S. 339)

Alle Ausprägungen von kooperativ-integrativen Projekten haben einen gemeinsamen Gegenstandsbereich, ein (meist lebensweltliches) Problem wie ,Nachhaltigkeit' oder eben auch ,sprachbewusster Fachunterricht', der mit Gewinn aus unterschiedlichen Perspektiven bearbeitet wird. Die Projekte unterscheiden sich hinsichtlich folgender Aspekte (vgl. Aboelela et al. 2007):

1. Grad der Integration in der interdisziplinären Forschung in Bezug auf gemeinsame Entwicklung von Theorien, Forschungsdesigns, Publikationstätigkeiten bzw. jeweilige Lernbereitschaft für die Sprache, Terminologie, Denkweisen bzw. der Literacy der Kooperationspartner.

2. Kontinuität der interdisziplinären Kooperation sowie die Zeit, die für Einarbeitung in die anderen Disziplinen bzw. Arbeitsfelder der Kooperationspartner sowie die Interaktion zwischen diesen Partnern investiert wird. Dabei soll auch 
eine gemeinsame Konzeptentwicklung, Publikations- und Disseminationstätigkeit betrieben werden.

3. Der Grad der Zusammenarbeit zeigt sich schließlich im Outcome in Bezug auf Problemlösungen, einer ,neuen' gemeinsamen Sprache, der Theoriebildung, aber auch im Erschließen neuer Forschungs- und Entwicklungsfelder. ${ }^{6}$

Mit dieser Begrifflichkeit und den damit verbundenen unterschiedlichen Konzepten und Strukturbedingungen wird in Fachbereichen wie Ökologie, Nachhaltigkeitsforschung, Medizin, Ingenieurswissenschaften, die sich schon länger mit Problemen der inter- und transdisziplinären Zusammenarbeit, den damit einhergehenden Reibungsverlusten, Missverständnissen etc. auseinandersetzen, auch darüber nachgedacht, welche Gelingensbedingungen für wirkungsvolle Inter- und Transdisziplinarität zu beachten sind.

Die Fachdidaktiken scheinen uns aufgrund ihrer ,inhärenten Interdisziplinarität' und ihrem Bezug zum Praxisfeld Schule für Inter- und Transdisziplinarität prädestiniert zu sein: Nicht nur haben sie in vielen Aspekten ihrer Disziplin im Sinne von Wittmann die gleichen Bezugsfelder, sondern sie haben aufgrund ihrer Ausrichtung auf ein Fach, ihrer ähnlichen Modellierung der Disziplinarität (teilweise gemeinsame Bezugsdisziplinen wie Lern- und Kognitionspsychologie, Soziologie, Pädagogik etc., Fokussierung auf einen Gegenstandsbereich und auf eine Schulpraxis und Bildungspolitik) einen ähnlichen Blick auf schulisches bzw. fachliches Lernen, dies gilt insbesondere für die zur Zeit in allen Fachdidaktiken diskutierte Kompetenzorientierung. Und schließlich haben sie auch ein gemeinsames transdisziplinäres Bezugsfeld - die Schule mit ihren fachlichen, fachübergreifenden und überfachlichen Kompetenzbereichen.

\section{Zur Ausgangslage: Mit Sprache im Fachunterricht lernen}

Nicht erst seit den PISA-Studien ist bekannt, dass fachliches Lernen eng mit sprachlichen Kompetenzen verbunden ist. ${ }^{7}$ Mit den PISA-Studien bekam diese

6 Zum Teil wird zum Outcome auch die Entwicklung der interdisziplinären Zusammenarbeit gezählt.

7 In England beispielsweise wurde der enge Zusammenhang von erfolgreichem fachlichen Lernen und Sprachkompetenzen bereits in den 70er Jahren erkannt: Alan Bullock (1975, S. 514) fordert entsprechend im Report of the Committee of Enquiry appointed by the Secretary of State for Education and Science (herausgegeben durch Her Majesty's Stationery Office): „Each school should have an organized policy for language across the curriculum, establishing every teacher's involvement in language and reading development throughout the years of schooling". 
Sichtweise jedoch im deutschen Sprachraum empirische Fundierung, denn sprachschwache Schüler und Schülerinnen wiesen auch in den Naturwissenschaften und in Mathematik schwächere Leistungen auf (bspw. Baumert/Schümer 2001; Leutner et al. 2004): Erfolgreiches fachliches Lernen ist stark von sprachlichen Kompetenzen abhängig: Nur wer die im Fachunterricht vermittelten Informationen versteht (durch Lesen oder Zuhören) und nur wer fähig ist, über fachliche Inhalte zu kommunizieren (schriftlich oder mündlich) und sein Verständnis anderen mitzuteilen, kommt im fachlichen Lernen voran und kann sein Fachwissen zeigen. Verschiedene Autoren weisen inzwischen darauf hin, dass die im Fachunterricht für das Lernen vorausgesetzten sprachlichen Kompetenzen bei vielen Schülern und Schülerinnen nicht vorhanden sind (vgl. u. a. Bolte/Pastille 2010; Morek/Heller 2012; Rincke 2010; von Borries 2011). So wird auch in der Nationalfondsstudie Textverstehen in den naturwissenschaftlichen Schulfächern ${ }^{8}$ eine Diskrepanz zwischen den bei den Schülern und Schülerinnen der Sekundarstufe I vorhandenen und den in Lehrmitteltexten geforderten Lesekompetenzen empirisch nachgewiesen (vgl. Schmellentin et al., im Druck). Problematisch ist eine solche Diskrepanz vor allem dann, wenn sie von den Lehrpersonen nicht bewusst wahrgenommen wird und die Schüler und Schülerinnen weder bei der Bewältigung fachlichen Lernens mittels Sprache unterstützt werden noch an den besonderen in den Schulfächern verwendeten sprachlichen Registern arbeiten. Dies führt zu Bildungsungleichheiten, von denen vor allem Lernende aus bildungsferneren Elternhäusern betroffen sind: Lernende, welche die im Fachunterricht vorausgesetzten sprachlichen Kompetenzen nicht aus ihrem außerschulischen Umfeld mitbringen, sind darauf angewiesen, diese im schulischen Umfeld unter Anleitung zu erwerben, denn sprachliche Defizite wirken sich in den Sachfächern kumulativ aus (vgl. Baumert/Schümer 2001).

Um der hier nur kurz skizzierten Problematik entgegenzuwirken und damit die sprachlich bedingten Bildungsungleichheiten abzuschwächen, ist die interdisziplinäre Zusammenarbeit von Sprach- und verschiedenen Fachdidaktiken nötig, wie die folgenden Ausführungen zeigen.

Die Ursache für die Diskrepanz von vorhandenen und geforderten Sprachkompetenzen im Fachunterricht kann sowohl bei den Anforderungen selbst als auch bei den Kompetenzen der Schüler und Schülerinnen liegen. Mit Blick auf das Textverstehen bedeutet dies: Verfügt ein Teil der Lernenden nicht über die für den Wissenstransfer vorausgesetzten Lesekompetenzen, muss entweder die

8 Das Projekt wird von Hansjakob Schneider und Claudia Schmellentin geleitet. Als Beraterin für naturwissenschaftsdidaktische Aspekte ist Anne Beerenwinkel am Projekt beteiligt. 
Sprache der Texte an die Verstehensmöglichkeiten der Lernenden angepasst oder die Lernenden müssen an die für das Verstehen der Texte notwendigen Handlungsweisen und Verstehensleistungen herangeführt werden. Die erste Forderung zielt darauf, sprachliche Hürden abzubauen, beispielweise durch eine sprachliche Vereinfachung von Texten. Bei der zweiten Forderung geht es darum, die Schüler und Schülerinnen sprachdidaktisch so zu unterstützen, dass sie sprachliche Hürden überwinden können.

Erste Ergebnisse aus dem oben angesprochenen SNF-Forschungsprojekt Textverstehen in den naturwissenschaftlichen Schulfächern weisen darauf hin, dass der Abbau sprachlicher Hürden z. B. durch Textvereinfachungen Grenzen aufweist. ${ }^{9}$ Einerseits lassen sich komplexe Inhalte nur bedingt mit einer einfacheren Sprache (mit einer einfachen Syntax oder gar einem Alltagswortschatz) vermitteln, andererseits ist bei der Konzeption eines sprachbewussten Fachunterrichts auch zu beachten, dass es nicht einfach eine Form einer in der Schule gepflegten und zum Zweck des Lernens herausgebildeten Sprache gibt (Feilke 2012), sondern dass die Sprache im Fachunterricht mit zunehmendem Lernalter auch fachspezifischer bzw. fachsprachlicher wird. Auf die Fachspezifik bildungssprachlicher Register weisen unter anderem Schulbuchanalysen hin (Kernen/Riss 2012; Nagy/ Townsend 2012; Schleppegrell 2004; Schrader 2013). Die Sprache wird von den Inhalten geprägt, die sie transferiert: Die Darstellung von Strukturen (z. B. Aufbau der Blüte), Prozessen (z. B. Fotosynthese), Funktionsweisen (z. B. Funktion der Schleimhäute in den Atemwegen) oder Modellen (z. B. Atommodelle), wie sie in den naturwissenschaftlichen Fächern üblich ist, erfordert andere sprachliche Mittel als die Darstellung von historischen Begebenheiten und Ereignissen, und zwar nicht nur in Bezug auf das Vokabular, sondern auch in Bezug auf die syntaktische und textuelle Struktur: Naturwissenschaftliche Texte weisen in Abhängigkeit zu ihren Inhalten und Funktionen fachsprachliche Merkmale auf wie hohe Informations- und Fachwortdichte, unpersönliche Ausdrucksweise, Auftreten von Grafiken/Bildern/Diagrammen usw. Texte für den Geschichtsunterricht ${ }^{10}$ hingegen sind aufgrund ihrer vielfältigen Funktionen sehr heterogen, meist weniger informationsdicht und weisen unter anderem vermehrt temporale und kausale Konnektoren sowie Mittel zum Ausdruck von Modalitäten auf, dies ebenfalls in Abhängigkeit von ihren Inhalten und Funktionen. Häufig wird eine Kernaussage

9 Dies obschon bei bestehenden Lehrmitteltexten durchaus Optimierungspotenzial vorhanden ist und fachlich unnötige sprachliche Hürden abzubauen sind (vgl. dazu detaillierter Schmellentin et al., im Druck).

10 Gemeint sind hier so genannte Darstellungstexte, nicht Quellentexte, die wiederum andere sprachliche Besonderheiten aufweisen können. 
mit mehreren Sätzen mit je leicht unterschiedlicher Perspektive eingekreist, wobei nicht alle Sätze für das Verstehen der Kernaussage gleich bedeutsam sind. Sie erfordern dafür von den Lesenden hohe Abstraktions- und Konkretisierungsleistungen (vgl. Lindauer et al. 2013; Schrader 2013, S. 45).

Unterschiedliche Textstrukturen machen auch unterschiedliche Be- und Verarbeitungsstrategien erforderlich: In einem Geschichtstext kann das Markieren wichtiger Inhalte zielführend für die Textbearbeitung sein, bei informationsdichten naturwissenschaftlichen Texten hingegen ist diese Strategie wenig hilfreich. Allgemeine Lesestrategien, wie sie auch im Deutschunterricht an den für den Deutschunterricht relevanten Inhalten vermittelt werden, sind für die Be- und Verarbeitung schulischer Texte in anderen Fächern nur bedingt hilfreich, da sie sich nicht immer übertragen lassen. Mit anderen Worten: Es geht nicht darum, die Schüler und Schülerinnen an ein überfachliches bildungssprachliches Register heranzuführen, sondern an mehrere fachsprachlich geprägte bildungssprachliche Register: Mit dem Erwerb von fachlichen Konzepten geht auch der Erwerb der damit verbundenen (fach-)sprachlichen Kompetenzen einher, entsprechend ist fachspezifische Sprachförderung Gegenstand aller Fächer und damit gleichzeitig auch Forschungsgegenstand aller Fachdidaktiken.

Sprachbewusster Fachunterricht sollte unter den bisher dargestellten Überlegungen folgende Eigenschaften aufweisen (vgl. Lindauer et al. 2013):

- Unnötige sprachliche Hürden werden zwar abgebaut, Texte werden bspw. vereinfacht, nicht aber ,ent-fachsprachlicht' oder gar simplifiziert: Nur wer mit Fachsprache in Kontakt kommt, kann auch die zum Fach gehörende Sprache erlernen.

- Schüler und Schülerinnen werden beim fachlichen Lernen beim Verstehen und Produzieren eines fachspezifischen Sprachgebrauchs unterstützt (bspw. indem das Verstehen gezielt und kleinschrittig mit Fragen zum Text angeleitet wird): Sprachabhängige Lernprozesse müssen auch sprachbewusst strukturiert werden.

- Der Aufbau fachsprachlicher Kompetenzen wird horizontal-fachübergreifend und vertikal-curricular durchgängig strukturiert.

Ein so definierter sprachbewusster Fachunterricht lässt sich nur in interdisziplinärer Zusammenarbeit von entsprechender Fachdidaktik und der Sprachdidaktik konzipieren und entwickeln. Dies illustriert ein Beispiel zum Abbau sprachlicher Hürden: Eine sprachliche Vereinfachung von Texten beispielsweise ist immer mit einer fachdidaktisch begründeten inhaltlichen Überarbeitung verknüpft. Dafür braucht es nicht nur sprachdidaktische, sondern in besonderem Maße fachdidaktische Expertise: Nur wer beurteilen kann, was der Inhalt und die unter Lernerperspektive relevante Information einer Textpassage, eines Satzes sein soll, kann eine Textpassage so reformulieren, dass sie verständlicher bzw. lernwirksamer 
wird. Würde man hier nur nach den aus der Textlinguistik bekannten Prinzipien für Textverständlichkeit vorgehen, würde die spezifische Fachlichkeit dieser Texte und damit auch ihr Potenzial für den Erwerb einer Fachsprache und für den Aufbau von Kompetenzen im Umgang mit Fachliteratur zerstört werden.

Trotz der beschriebenen fachlichen Bedingtheit von sprachbewusstem Fachunterricht plädieren wir dafür, diesen so weit als möglich auch fachübergreifend zu konzipieren, denn aus Schülersicht wäre eine kohärente Modellierung von Sprachkompetenzen und deren Aufbau im schulischen Unterricht über möglichst alle Fächer hinweg wünschenswert. Wie die Betonung auf fachübergreifende Merkmale von Bildungssprache ohne den Verlust der fachspezifischen Merkmale bewerkstelligt werden könnte, wird am Beispiel von Lesestrategien gezeigt: Der komplexe Lese- bzw. Verstehensprozess ließe sich auf einer Metaebene in allen Fächern in gleiche Schritte strukturieren. Im hier vorgestellten Projekt haben wir uns dazu auf das in der Schweiz verbreitete Sprachlehrmittel Die Sprachstarken (Lindauer/Senn 2008-2016) gestützt, welches den Leseprozess in folgende vier Schritte strukturiert:

Leseschritt 1: dem Text begegnen - Vorwissen aktivieren, Leseerwartung aufbauen, Ziele klären

Leseschritt 2: den Text bearbeiten - lokale Informationen gewinnen

Leseschritt 3: Textinhalte verarbeiten - Textinhalte miteinander verknüpfen

Leseschritt 4: Textverständnis überprüfen und mit Vorwissen in Verbindung bringen

Die explizite Strukturierung in die immer gleichen vier Schritte soll die Schüler und Schülerinnen dazu führen, den komplexen Leseprozess mit der Zeit selbst zu steuern. Für eine kohärente Förderung von Lesekompetenzen ist es wünschenswert, wenn der Leseprozess in allen Fächern auf der Ebene dieser vier Schritte gleich strukturiert würde. Die Ebene der konkreten Handlungen beim Lesen spezifischer Fachtexte hingegen, die durch die einzelnen Leseschritte ausgelöst werden, variiert - wie oben gezeigt - je nach Fach und Textbeschaffenheit. Mit anderen Worten: Eine durchgängige Sprachförderung und Konzeption von sprachbewusstem Unterricht muss auch nach Möglichkeiten fachübergreifender Aspekte des Verhältnisses von Fachlernen und Sprache fragen. Diese Fragen lassen sich nur in interdisziplinärer Zusammenarbeit der verschiedenen Fachdidaktiken klären.

$\mathrm{Zu}$ einer durchgängigen Konzeption von sprachbewusstem Fachunterricht gehört allerdings nicht nur deren horizontale Modellierung über die Fächer hinweg, sondern auch die vertikale, die über die Schulstufen hinweg geht. Damit kommt zu den oben genannten Merkmalen von sprachbewusstem Unterricht noch das Folgende hinzu: 
- Die sprachdidaktischen Maßnahmen, um sprachlich bedingte Lernprozesse zu strukturieren, werden über die Fach- und Stufengrenzen harmonisiert (z. B. Strukturierung von Lese- und Schreibprozessen in immer gleiche Schritte).

Spätestens dieses Merkmal bedingt auch eine Ausweitung der Zusammenarbeit: Die Konzeption von sprachbewusstem Fachunterricht kann gleich wie deren Umsetzung nicht an die Sprach- und Fachdidaktiken alleine bzw. an die Sprach- und Fachlehrpersonen delegiert werden. Eine durchgängige Konzeption auch über die Fach- und Stufengrenzen hinweg bedingt eine Planungs- und Koordinationsinstanz sowie der Schaffung geeigneter Rahmenbedingungen. Dies macht die Zusammenarbeit mit dem Praxisfeld, so beispielsweise Bildungssteuerungsverantwortlichen, Schulleitungen, Sprachverantwortlichen an Schulen notwendig.

\section{Transdisziplinäre Projektanlage und Ergebnisse}

Aus der oben skizzierten Problemlage war das auf den Naturwissenschaftsunterricht fokussierte Projekt Fachlernen und Sprache von Anfang an interdisziplinär, aber nur in einem Teilaspekt transdisziplinär angelegt. ${ }^{11}$ Mit dem Projekt wurden folgende Ziele verfolgt und Produkte entwickelt, die auf www.zentrumlesen.ch zum Download bereitstehen:

- Lehrpersonen sollen ihren Fachunterricht sprachbewusster gestalten können.

- Schulen sollen einen sprachbewussten Unterricht über die Fachgrenzen hinweg gestalten können.

- Lehrmittel für den Fachunterricht sollen sprachbewusst gestaltet werden.

- Die Implementierung der fachdidaktischen Entwicklungen in den Schulen und im Unterricht soll mitgeplant werden.

In mehreren (transdisziplinären) Diskussionen zwischen Akteuren aus der Deutschdidaktik, der Naturwissenschaftsdidaktik und der Bildungsplanung wurden die Inhalte für einen Auftrag der vier Kantone an die Pädagogische Hochschule bzw. ans Zentrum Lesen formuliert - Akteure aus dem schulischen Feld waren hier nicht beteiligt. Es wurden dabei folgende Rahmenbedingungen definiert:

a) In einem interdisziplinären Team von Deutschdidaktik und Naturwissenschaftsdidaktik sollten die im Folgenden vorgestellten Produkte entwickelt werden.

11 Transdisziplinäre Projekte sind organisatorisch und konzeptionell ausgesprochen anspruchsvoll (vgl. Axelsson 2010); hier besteht für die Deutsch- bzw. für die Fachdidaktik(en) noch einiger Bedarf an Erfahrungen und theoretisch-konzeptionellen Reflexionen. 
b) Die Produkte sollten dann in einer zweiten Projektphase mit verschiedenen, nicht nur in der Steuerungsgruppe bereits aktiven Akteuren aus der Bildungsplanung sowie einigen geladenen Experten kritisch unter den unterschiedlichen Akteursperspektiven diskutiert werden.

c) Im Anschluss an die daraus resultierende Überarbeitung sollten die neuen Materialien mit einer stufengemischten Gruppe von Lehrpersonen diskutiert werden. Die Lehrpersonen sollten zudem die Materialien auch im Unterricht erproben.

d) Die nach der Erprobung überarbeiteten Produkte würden dann von der Bildungsplanung für die Dissemination durch Weiterbildungsangebote für sog. SWiSE-Schulen ${ }^{12}$ sowie zum Aufschalten auf der Website des Zentrums Lesen freigegeben.

Die folgende Darstellung der Produkte ${ }^{13}$ dient vor allem dazu, die multi-, interund transdisziplinären Arbeitsphasen in diesem Projekt und die Aufgaben der verschiedenen Akteursgruppen aus der Deutsch- und Naturwissenschaftsdidaktik sowie der Bildungsplanung und den Lehrpersonen, welche erst gegen Ende des Projekts involviert wurden, aufzuzeigen.

\subsection{Handreichung Sprachbewusst unterrichten - Eine Unterrichtshilfe}

Das Hauptziel des Projekts war es, Konzepte und Materialien zu entwickeln, damit (Fach-)Lehrpersonen ihre Schülerinnen und Schüler sprachbewusst im Fachlernen unterstützen können. Dafür müssen Lehrpersonen über ein sprachdidaktisches Wissen sowie über ein Repertoire an Handlungsoptionen und mustergebenden Unterrichtsbeispielen verfügen, um sprachliche Hindernisse auf dem Weg zum Fachlernen erkennen und sprachbewusst modellieren zu können. Die Handreichung wurde daher in drei Teile gegliedert:

(1) Im ersten Teil wird das Konzept eines sprachbewussten Fachunterrichts für die Sprachhandlungsdomänen Lesen und Schreiben sowie Mündlichkeit inkl.

12 Im Kontext der Etablierung der nationalen Bildungsstandards in der Schweiz, eines neuen sprachregionalen Lehrplans für die Deutschschweiz und auch mit Bezug auf den (bildungs-)politisch geführten Diskurs eines sog. Fachkräftemangels im naturwissenschaftlich-technischen Bereich lief von 2011-2015 das Projekt Innovation SWiSE (Swiss Science Education/Naturwissenschaftliche Bildung Schweiz), an dem rund 60 Schweizer Schulen teilnahmen, um die naturwissenschaftliche Ausbildung zu stärken.

13 In einem damit zusammenhängenden, vom Kanton Aargau finanzierten Projekt wurden auch eine Analyse und Empfehlung für die sprachbewusste Gestaltung von Lehrmitteln für den naturwissenschaftlichen Unterricht vorgenommen, die hier nicht dargestellt werden (vgl. Kernen/Riss 2012; Lindauer et al. 2012). 
Unterrichtsgespräch und dem dazu querliegenden Wortschatz möglichst knapp und übersichtlich dargestellt. Dieser erste Teil wurde vom deutschdidaktischen Teilteam entwickelt. Danach haben ihn Naturwissenschaftsdidaktikerinnen gegengelesen, Unverständliches markiert und auf fachliche Problemstellen hingewiesen. Nach mehreren diskursiven Durchgängen wurde der Text in einer gemeinsamen Fassung den Akteurinnen aus der Bildungsplanung zum Lesen und Prüfen gegeben. Dieser Teil wurde also in einer nur ansatzweise inter- und transdisziplinären Arbeitsweise erstellt. Aboelela et al. (2007) sprechen denn auch bei solchen Arbeitsformen von multidisziplinären Zugangsweisen, weil hier nicht gemeinsam entwickelt und konzipiert wird, sondern jede Akteursgruppe aus ihrer Perspektive Teile für das Gemeinsame beisteuert. Angesichts der hohen sprachdidaktischen Fachlichkeit, die dieser erste Teil erfordert, schien uns diese Arbeitsweise am effizientesten. Mit anderen Worten: Nicht alle Arbeitsphasen in einem inter- oder transdisziplinären Projekt müssen im engeren Sinn interdisziplinär sein, sondern es ist jeweils genau zu prüfen, in welchen Phasen arbeitsteilig und sukzessive und in welchen inter- oder gar transdisziplinär entwickelt bzw. geforscht wird.

(2) Auch für den zweiten Teil der Handreichung wurde zuerst multidisziplinär gearbeitet. In diesem Teil der Handreichung finden sich fachspezifische Checklisten für die Lehrpersonen, welche die im Theorieteil dargestellten domänenspezifischen Lese- und Schreibstrategien auf einem DIN-A4-Blatt darstellen, sodass die Lehrperson im Unterricht den einzelnen Punkten folgen und ähnlich wie auf einer Checkliste abarbeiten kann (vgl. Abb. 1).

\section{Abb. 1: Ausschnitt aus einer Checkliste aus der Handreichung «Sprachbewusst} unterrichten» (Lindauer et al. 2012)

\section{Checkliste «Lesen informationsdichte Texte» (vor allem Naturwissenschaften)}

Hilfestellungen durch Lehrperson

Leseschritt 1: Vorwissen aktivieren, Leseerwartung aufbauen, Ziele klären

- Leseerwartung aufbauen, Funktion des Textes erfassen

- Vorwissen aktivieren

- Text überblicken: anhand der (Unter-)Titel, Bilder/Grafiken/Diagramme erkennen, worum es im Text geht (Thema)

- erstes schnelles Durchlesen, sich im Text orientieren.

Für Recherchen:

- anhand der (Zwischen-)Titel etc. beim Überfliegen feststellen, ob der Text Informationen zum gewünschten Thema enthält

LP wählt angemessene Hilfestellungen aus:

- Textfunktion und Kerninhalt explizieren: Der Text erläutert, wie XY aufgebaut ist / funktioniert; den Text könnt ihr zur Repetition der letzten Stunde/als Prüfungsvorbereitung nutzen.

- Vorwissensaktivierung anregen: z. B. Vorwissen in Stichworten (2-3) notieren lassen, Begriffe an WT festhalten (evtl. Cluster mit wichtigsten Begriffen erstellen lassen (in PA), Begriffsnetze in PA oder im Plenum verbalisieren)

- Text überblicken lassen: Auftrag zum Überblicken bzw. ersten schnellen Durchlesen des Textes erteilen: (Unter-)Titel, Bilder/Grafiken/Diagramme, Auffälliges anschauen lassen. Vermutungen, worum es im Text 
In einer ersten Phase wurden solche Checklisten auch für Schülerinnen und Schüler entwickelt. Es zeigte sich jedoch, dass solche eher abstrakten Listen für diese wenig hilfreich sind. Eine Ausnahme bildete das Präsentieren, bei dem je eine Checkliste für Lehrperson und eine für Lernende erstellt wurde. Sinnvollerweise würden solche Listen auch im transdisziplinären Zusammenspiel von Sprachund Naturwissenschaftsdidaktik sowie mit Lehrperson und Schülergruppen entwickelt, dafür war aber das zeitliche wie auch das finanzielle Budget zu knapp bemessen.

\section{Abb. 2: Ausschnitt aus je einer Checkliste für Lehrperson und Schülerinnen und Schüler aus der Handreichung «Sprachbewusst unterrichten» (Lindauer et al. 2012)}

\section{Checkliste «Präsentieren» (Lehrperson)}

Schüleraktivität

Hilfestellungen durch Lehrperson

Schritt 0: Vorbereitungsphase, Organisation und Ideenfindung

- Themenwahl treffen

- Erwartung explizieren, Beurteilungskriterien mit
S vorbesprechen bzw. gemeinsam entwickeln
(max. 7 Kriterien)
- Themenwahl wenn nötig unterstützen

Schritt 1: Wahl und Bearbeitung des Themas

- Informationen zum Thema sammeln, Informationen überfliegen, Stichworte zu Informationsquelle verfassen und in Sammelmappe ablegen (evtl. Linksammlung anlegen)

- Informationen ordnen (wichtig, weniger wichtig, thematische Ordnung (Nummerierungen, Stichworte, Mind-Map/Cluster)

- Wichtige Informationsquellen genau lesen, Infor-

- Mit präzisen Aufträgen strukturieren: Such im Internet Informationen zum Thema, gib die Stichwörter $X Y$ ein. Überflieg die Informationen der verschiedenen Informationsquellen, notier in Stichworten, worum es geht ...

- Informationsquellen eingrenzen, überschaubare und für $S$ bewältigbare Anzahl von Büchern oder Links vorgeben

\section{Checkliste «Präsentieren» (für SchülerInnen)}

\section{Schritt 1: Wahl und Bearbeitung des Themas oder eines Buches}

Thema auswählen, Informationen zum Thema sammeln, Informationen überfliegen, Stichworte zu Informationsquelle verfassen und in Sammelmappe ablegen (evtl. Linksammlung anlegen) Informationen ordnen (wichtig, weniger wichtig, thematische Ordnung (z.B. Nummern zu Themenbereiche, Stichworte, Mind-Map/Cluster))

Wichtige Informationsquellen genau lesen, Informationen festhalten (Stichworte, Notizen, Zusammenfassungen ...), ordnen und in Sammelmappe ablegen passende Gegenstände, Bilder, Erfahrungen, Zitate zu einem Thema organisieren, die man zeigen möchte, in Sammelmappe ablegen Ideen zum Ablauf der Präsentation sammeln und in Sammelmappe ablegen Sammelmappe mit LP besprechen 
Zudem zeigt sich, dass solche Checklisten wegen ihrer hohen Fachspezifik (vgl. Abschnitt 2) auf der einen Seite und dem hohen Anspruch an sprachdidaktisches Wissen andererseits nur pluri-interdisziplinär entwickelt werden sollten. Dafür hätte man aber mit weiteren Fachdidaktiken zusammenarbeiten müssen, was wiederum den Komplexitätsgrad des Projekts (zu) stark erhöht hätte.

$\mathrm{Zu}$ einer engen interdisziplinären Arbeit zwischen Sprach- und Naturwissenschaftsdidaktik kam es dann nach einer von der Bildungsplanung bzw. vom Bildungsraum Nordwestschweiz organisierten Klausur, mit weiteren Akteuren aus der Bildungsplanung. Die endgültigen Produkte wurden nochmals mit der Begleitgruppe aus der Bildungsplanung diskutiert. Leider konnten - wie oben schon gesagt - keine Lehrpersonen in die Entwicklung dieser Listen involviert werden. In anderen Projekten - wie bspw. bei der Entwicklung des Lehrmittels Die Sprachstarken oder bei der Ausarbeitung von Beurteilungsinstrumenten - hat sich eine enge Zusammenarbeit zwischen Fachdidaktik und Lehrperson bei der Entwicklung von Unterrichtsmaterialien und -hilfen als ausgesprochen fruchtbar erwiesen. In diesem Projekt standen dafür leider weder die zeitlichen noch die finanziellen Ressourcen zur Verfügung (vgl. dazu die Ausführungen unten).

(3) Im dritten Teil der Broschüre werden die Konzepte und Strukturierungshilfen mit Aufgabenbeispielen - in der Schlussfassung nur fürs Lesen - illustriert. Diese Arbeit wurde von Anfang an interdisziplinär konzipiert: Deutschdidaktikerinnen arbeiteten gemeinsam mit Kolleginnen aus der Naturwissenschaftsdidaktik an den Aufgaben. ${ }^{14}$ Die Arbeit an den Aufgabenbeispielen zeigte dabei deutlich, wie wichtig die gemeinsame Arbeit der Fachdidaktiken ist (im Gegensatz zu einer sukzessiven, bei der zuerst Naturwissenschaftler die Texte verfassen und danach die Sprachdidaktiker sie ,optimieren'). So konnte bspw. von Seiten der Sprachdidaktik eingebracht werden, dass Fragen zum expliziten Textverstehen gestellt werden sollten, die bestimmte Textstellen fokussieren. Sprachlich schwierige Stellen konnten bestimmt, fachlich und sprachlich analysiert und Vorschläge für gut verständliche Fragen gemeinsam formuliert werden. Von Seiten der Naturwissenschaftsdidaktik wurde definiert, welche Begriffe und Konzepte bzw. welche Textstellen oder Abbildungselemente zentral sind, in welcher Reihenfolge sie für den Konzeptaufbau benötigt und entsprechend von den Schülern und Schülerinnen erschlossen werden müssen, welche Sätze Misskonzepte stärken könnten oder welche alternativen Formulierungen es für die Konzepte in den

14 Die Aufgabenbeispiele zum Textverstehen beziehen sich auf die drei naturwissenschaftlichen Fächer Biologie, Physik und Chemie und basieren größtenteils auf Texten gängiger Lehrmittel der Sekundarstufe I (7.-9. Schuljahr). Sie wurden von Claudia Hefti und Anne Beerenwinkel entwickelt. 
Fragestellungen geben könnte. Auf diese hier nur skizzierte Art profitierte die Aufgabenformulierung auf allen Ebenen von dem mehrfachen und aufeinander Bezug nehmenden Austausch zwischen den Fachdisziplinen.

Fazit: Die Arbeit an der Handreichung für einen sprachbewussten Fachunterricht hat gezeigt, wie wertvoll eine interdisziplinäre Arbeit zweier Fachdidaktiken sein kann. Die Arbeit hat auch gezeigt, dass nicht jede Phase interdisziplinär i. e. S. sein muss, dass jeweils das Maß der Interdisziplinarität funktional, aber auch ressourcenschonend bedacht sein will.

Als fruchtbar hat sich auch die transdisziplinäre Zusammenarbeit mit der Bildungsplanung erwiesen, auch wenn diese Arbeitsphase zusätzliche Zeitressourcen gekostet hat, waren hier die vielfältigen Feedbacks, aber auch die gemeinsame Konzipierung der Handreichung ein Gewinn. Leider konnte nicht auch noch mit einer Gruppe von Lehrpersonen transdisziplinär gearbeitet werden: Eine reine Feedback-Runde bzw. eine Fragebogenerhebung bei Lehrpersonen von bereits mehr oder weniger fertig gestellten Produkten liefert zwar noch einige wertvolle Hinweise für die Endredaktion, aber die Akzeptanz und v. a. die Praxistauglichkeit könnte durch ein echt transdisziplinäres Arbeiten von Anfang an deutlich erhöht werden (vgl. Abschnitt 3.3).

\subsection{Sprachprofile}

Die Sprachprofile wurden 2006 vom Kanton Basel-Stadt herausgegeben. Sie wurden von verschiedenen stufenbezogenen Lehrpersonen-Teams unter der Leitung von Claudio Nodari entwickelt und sollten im Rahmen des hier vorgestellten Projekts überarbeitet und an die Konzeption eines sprachbewussten Fachunterrichts angepasst werden. Mit den (überarbeiteten) Sprachprofilen sollte allen Lehrpersonen (nicht nur denjenigen, die Sprache unterrichten) deutlich gemacht werden, welche sprachlichen Anforderungen schulisches Lernen stellt und wie dieses Lernen mithilfe von sprachlichen Strategien unterstützt werden kann. Gedacht wird dabei an eine,Arbeitsteilung' zwischen Deutschunterricht und den anderen Fächern, die darin besteht, dass im Deutschunterricht die relevanten sprachlichen Strategien kleinschrittig eingeführt werden, da dort der Ort und die Zeit ist, diese Strategien vertiefend zu reflektieren. Aufgabe der anderen Fächer ist es dann, die für den eigenen Fachkontext nötigen Sprachstrategien aufzugreifen und die Lernenden sprachlich so zu unterstützen, dass sie dem fachlichen Unterricht folgen können. Diese Aufgabenverteilung muss zwischen den Fächern koordiniert werden, soll ein curricular abgestimmtes Lernen ermöglicht werden. Auch hier sollen die Sprachprofile Unterstützung bieten. 
Dafür werden diejenigen Dimensionen sprachlicher Aktivitäten, die fürs Lernen in den Fächern zentral sind, stufenweise mithilfe von Deskriptoren dargestellt. Die ursprünglichen wie auch die neuen Sprachprofile sind daher ein stufen- und fächerübergreifendes Planungsinstrument für eine sprachliche Förderung in allen Fächern - fokussiert auf diejenigen Sprachkompetenzen, die für ein fachliches Lernen relevant sind. Neu sind sie auch als ein Instrument, das den (Fach-) Lehrpersonen deutlich macht, inwiefern die Sprache Hilfe bei der Vermittlung des Fachinhalts bieten kann bzw. wie Fachinhalte sprachlich didaktisiert werden müssen, damit sie das Lernen befördern. Die Sprachprofile liegen dafür in zwei Versionen vor: (1) „Sprachliches im Unterricht - Deskriptoren und Hinweise“ und (2) „Sprachliches im Unterricht - Deskriptoren und Planungshilfe“.

(1) „Sprachliches im Unterricht - Deskriptoren und Hinweise“: In einer zweispaltigen Tabelle sind in der linken Spalte die fürs fachliche Lernen besonders relevanten Deskriptoren für die einzelnen Sprachhandlungen formuliert. In der rechten Spalte werden im Sinne eines reduzierten Kommentars einige wenige Hinweise zu den Deskriptoren gegeben. Ausführlich dargestellt und sprachdidaktisch erläutert sind die einzelnen Sprachhandlungsdomänen in der im vorhergehenden Abschnitt dargestellten Handreichung «Sprachbewusst unterrichten».

Abb. 3: Ausschnitt aus den „Sprachprofilen“ (Lindauer et al. 2012)

\begin{tabular}{|c|c|}
\hline $\begin{array}{l}\text { Deskriptoren eLesens } \\
\text { Die Lernenden werden im Unterricht zu Folgendem angeleitet: }\end{array}$ & Hinweise zu den Deskriptoren \\
\hline $\begin{array}{l}\text { Grundfertigkeiten } \\
\text { - Leseflusssigkeit (Fluency) weiterentwickeln. }\end{array}$ & 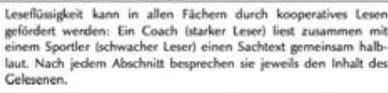 \\
\hline $\begin{array}{l}\text { Narrativen Sachtexten das Wesentliche entnehmen } \\
\text { - einen Text selbstăndig still und angemessen flussig lesen. } \\
\text { - einen Text überfliegen und im Text orientierende Elemente beachten (Zwischentitel, Illustratio- } \\
\text { nen, fett Gedrucktes), daraus Vermutungen 0ber den Textinhalt anstellen (Leseschritt 1). } \\
\text { - Einzelinformationen, die von der Lehrperson erfragt werden, in Textabschnitten suchen und } \\
\text { markieren (Leseschritt 2). } \\
\text { - in einem Bericht die wichtigsten Personen und Ereignisse erkennen und benennen. } \\
\text { - eine Handlung und Ereignisabfolge verstehen und z. B. in einer Bildfolge ordnen. } \\
\text { - in einem Text weiterlesen, auch wenn man noch nicht alles verstanden hat (orientierendes Le- } \\
\text { sen). }\end{array}$ & 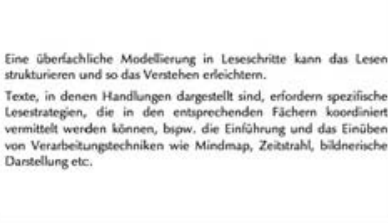 \\
\hline
\end{tabular}

(2) „Sprachliches im Unterricht - Deskriptoren und Planungshilfe“: Da der bewusste Umgang mit Sprachkompetenzen nicht nur Aufgabe des Deutschunterrichts ist, sondern sprachbewusster Unterricht in allen Fächern relevant ist, sollen die Deskriptoren auch dazu dienen, dass Schulhaus- und Fachteams sowohl horizontal als auch vertikal koordinieren können, in welchem Fach zu welcher Zeit welche sprachliche Aktivität besonders fokussiert wird (vgl. dazu auch Abschnitt 2). Dahinter steht die Vorstellung, dass im Deutschunterricht bspw. eine spezifische Lesestrategie eingeführt und mit den Schülern und Schülerinnen reflektiert wird 
und dass zeitnah dazu im Fachunterricht die im Deutschunterricht erarbeiteten Lesetechniken und -strategien auf möglichst gleiche Weise aktiviert und sprachdidaktisch begleitet werden: Dafür sind Absprachen zwischen den Fächern nötig, und zwar auch hinsichtlich der sprachdidaktischen Unterstützung von Sprachhandlungen im Fachunterricht.

Die neuen Sprachprofile wurden monodisziplinär von der Deutschdidaktik überarbeitet. Dadurch entstand zwar eine Kohärenz des sprachlichen Lernens über die einzelnen Schulstufen hinweg (durchgängige Sprachförderung), für die konzeptionelle Weiterentwicklung wäre aber auch hier eine interdisziplinäre $\mathrm{Zu}$ sammenarbeit mit verschiedenen Fachdidaktiken, aber auch eine transdisziplinäre Arbeit mit Lehrpersonen aus den verschiedenen Stufen und Fächern und möglicherweise auch die Zusammenarbeit mit Schulleitungen fruchtbar, aber auch sehr aufwendig gewesen: So sinnvoll eine transdisziplinäre Konzeption sein kann, so schnell stößt man nicht nur an finanzielle, sondern insbesondere an organisatorische Grenzen - die transdisziplinäre Zusammenarbeit so vieler Akteure ließe sich wohl nur in einem mehrstufigen Projekt gestalten. Leider standen dafür weder die Ressourcen für eine pluri-interdisziplinäre Arbeit zwischen mehreren Fachdidaktiken noch die für eine transdisziplinäre Zusammenarbeit mit stufenübergreifenden Schulhausteams und den entsprechenden Fachgruppen zur Verfügung.

Für die Etablierung solcher Instrumente in der schulischen Praxis wären zudem neben Einführungskursen zu den Instrumenten auch sprachliche Fachpersonen bzw. Sprachbeauftragte in den Schulen zu etablieren. Dafür müssten aber an den PHs entsprechende Studienangebote entwickelt werden. Auch hier wäre dann ein transdisziplinäres Zusammenspiel der verschiedenen Akteure zu etablieren. Insbesondere müssten hier auch die Bildungsplanung und die Standesorganisation der Schulleitungen involviert sein.

\subsection{Hinweise zur Dissemination und Umsetzung}

Die sich in Entwicklung befindlichen Materialien wurden in unterschiedlichen Gruppen mehrfach kritisch diskutiert. Dadurch sollte eine Transdisziplinarität das Projekts etabliert werden: Nicht nur zwischen den beiden Fachdidaktiken, sondern auch zwischen Fachdidaktik und Bildungsplanung sowie Fachdidaktik und Unterrichtspraxis sollte zusammengearbeitet und von den je eigenen Sichtweisen für das Projekt profitiert werden.

Im Frühjahr 2013 war dieser Qualitätsprozess weitgehend abgeschlossen. Das Interesse der Praxis an einem sprachbewussten Unterricht hielt sich jedoch in sehr engen Grenzen: Insgesamt nahmen nur 18 Lehrperson an einem Hearing teil und nur noch sechs davon an der schriftlichen Evaluation der gesamten Materialien. Es 
wurde nach der Verständlichkeit der Handreichung (Struktur, Sprache, Umfang) sowie der Nützlichkeit der darin aufgeführten Beispielen - das Textverstehen leitende Fragen zu Lehrmitteltexten - gefragt sowie darum gebeten, die Checklisten zu erproben. Die Rückmeldungen waren zwar durchgehend positiv, das muss aber nicht nur an der Qualität der Materialien liegen, sondern kann auch damit erklärt werden, dass die Teilnehmenden dem ganzen Unterfangen sehr positiv gegenüber standen. Die Checklisten selbst wurden nur von einer Person erprobt. ${ }^{15}$

Die Erprobung, aber auch das hier nicht dargestellte von den SWiSE-Schulen ungenutzte Angebot von Weiterbildungsveranstaltungen hat gezeigt, dass in der Praxis der naturwissenschaftlichen Fächer nur eine sehr geringe Sensibilisierung für die Problematik eines sprachbewussten Fachunterrichts vorhanden ist. Zum einen scheint sprachlichen Aspekten wenig Bedeutung für das fachliche Lernen beigemessen zu werden (mit Ausnahme der Themen Deutsch als Zweitsprache und Fachwortschatz im engeren Sinn), zum anderen wird Lesen oder gar Schreiben im naturwissenschaftlichen Unterricht oft mit ,papierenem' Unterricht als Gegenpol zu einem handlungsorientierten Unterricht gleichgesetzt. Dies widerspricht zwar sowohl den empirischen Erkenntnissen zur Bedeutung von Sprache für fachliches Lernen als auch der Bedeutung sprachlicher Handlungen - inkl. Lesen und Schreiben - für einen erfolgreichen forschend-entdeckenden Unterricht.

Es hat sich hier also einmal mehr gezeigt, dass die Zusammenarbeit mit der Schulpraxis nicht auf das nachgeschaltete Erproben von bereits mehr oder weniger fertig entwickelten Produkten gelegt werden darf, sondern dass die Schulpraxis im Sinne einer engen Transdisziplinarität von Anfang an, in unterschiedlichen Phasen des Projekts mehr oder weniger eng, an der Entwicklung beteiligt sein muss. Dadurch gewinnt das Projekt nicht nur an fachlicher und schulpraktischer Qualität, sondern dadurch können sich Lehrpersonen auch fachlich weiterentwickeln und stehen dann bei der Dissemination als Multiplikatoren bzw. als Multiplikatorinnen zur Verfügung.

15 Das geringe Interesse der Praxis erklärt sich möglicherweise auch daraus, dass die Lehrpersonen zurzeit drängendere Probleme haben: Auf der Sekundarstufe I ist dies eine Umstrukturierung in zwei Kantonen des Bildungsraums. Zudem beschäftigen Fragen zum sog. ,kompetenzorientierten Unterricht' im Zuge des Lehrplans 21 die Lehrpersonen stark (vgl. http://www.lehrplan.ch. Abgerufen am 16.03.2016). 


\section{Fazit}

Trotz all der oben skizzierten Schwierigkeiten hat sich im Kontext dieses Projekts gezeigt, dass der eingeschlagene Weg, nämlich die grundsätzliche Inter- und Transdisziplinariät solcher Projekte, erfolgsversprechend ist. So hat sich eine an der Informationsveranstaltung ausgesprochen kritisch äußernde Lehrperson nach dem Einsatz des verteilten Aufgabenmaterials sehr positiv geäußert: „Gerne bestätige ich, dass die Impulse aus dem Kurs in meinen Unterricht eingeflossen sind und ich auch feststellen konnte, wie gut viele Realschülerinnen und -schüler auf die Leittexte einsteigen und dabei wirklich erstaunliche Resultate liefern. " Diese positiven Resultate lassen sich auf die intensive und enge interdisziplinäre Zusammenarbeit von Naturwissenschaftsdidaktik und Sprachdidaktik zurückführen, bei der inhaltliche Aspekte mit sprachdidaktischen verschränkt wurden.

Die Schule hat in Bezug auf (fachspezifisches) Sprachlernen, aber auch in Bezug auf Ermöglichung von Fachlernen durch Abbau unnötiger sprachlicher Barrieren einen gemeinsamen Bildungsauftrag. Dieses Denken in einem Gesamtauftrag der Schule fällt insbesondere spezialisierten Fachlehrpersonen schwer bzw. wurde ihnen in ihrer Ausbildung kaum vermittelt. Dass ein sprachbewusster Fachunterricht auch einen Auftrag an die Fachdidaktiken in der Grund- und Weiterbildung von Lehrpersonen beinhaltet, muss ebenfalls erst noch etabliert werden: Seminare und Weiterbildungsangebote für Sachfächer fehlen noch weitgehend.

Die Umsetzung eines sprachbewussten Fachunterrichts kann also nicht an die Lehrpersonen alleine delegiert werden: Alle Akteure des Bildungssystems - Fachdidaktiken, Bildungsplanung, Schulen - müssen ihren Anteil an der Umsetzung leisten: So müssen beispielsweise Ressourcen für Weiterbildung, Entwicklung von Unterrichtshilfen usw. zur Verfügung gestellt werden, schulintern müssen Sprachfördermaßnahmen koordiniert werden können, die Thematik muss in die Lehrerbildung integriert werden usw. Sprachbewusster Fachunterricht ist damit Aufgabe des gesamten Bildungssystems und nicht nur einzelner Akteure.

Und so wie die Schule einen über die Fächer hinausweisenden Bildungsauftrag hat, sollten auch die Fachdidaktiken ein nicht nur fachspezifisches, sondern ein darüber hinausweisendes Bildungskonzept in interdisziplinären Kooperationen mit den anderen Fachdidaktiken, aber auch mit anderen Disziplinen der Bildungsforschung elaborieren. Insbesondere für Entwicklungsprojekte lohnt sich eine transdisziplinäre Projektanlage, bei der Akteure aus der Schulpraxis und der Bildungsplanung von Anfang an in die Entwicklungen aktiv involviert sind. Es gilt also nicht nur fachübergreifende Unterrichtsmodelle zu entwickeln und Strukturen für fachübergreifendes Lernen zu schaffen, sondern es gilt auch im transdisziplinären Zusammenspiel aller an Schule Beteiligter überfachliche Kompetenzen auszubilden. 


\section{Literatur}

Adame, Domingo (2011): From a Disciplinary to a Transdisciplinary Vision of the University: A Space of Knowledge, Culture, Art, Spirituality, and Life. In: Transdisciplinary Journal of Engineering \& Science. Vol. 2, pp. 33-29.

Aboelela, Sally W./Larson, Elaine/Bakken, Suzanne/Carrasquillo, Olveen/Formicola, Allan/Glied, Sherry A./Haas, Jane/Gebbie Kristine M. (2007): Defining Interdisciplinary Research: Conclusions from a Critical Review of the Literature. In: Health Service Research. Vol. 42. No. 1, pp. 329-346.

Axelsson, Robert (2010): Integrative research and transdisciplinary knowledge production: a review of barriers and bridges. In: Journal of Landscape Ecology. Vol. 4. No. 2, pp. 14-40.

Baumert, Jürgen/Schümer, Gundel (2001): Familiäre Lebensverhältnisse, Bildungsbeteiligung und Kompetenzerwerb. In: Baumert, Jürgen/Klieme, Eckhard/Neubrand, Michael/Prenzel, Manfred/Schiefele, Ulrich/Schneider, Wolfgang/Stanat, Petra/Tillmann, Klaus-Jürgen/Weiß, Manfred (Hrsg.): PISA 2000. Basiskompetenzen von Schülerinnen und Schülern im internationalen Vergleich. Opladen: Leske \& Budrich, S. 323-410.

Bogner, Alexander/Kastenhofer, Karen/Torgensen, Helge (Hrsg.) (2010): Interund Transdisziplinarität im Wandel? Neue Perspektiven auf problemorientierte Forschung und Politikberatung. Baden-Baden: Nomos.

Bolte, Claus/Pastille, Reinhard (2010): Naturwissenschaften zur Sprache bringen. Strategien und Umsetzung eines sprachaktivierenden naturwissenschaftlichen Unterrichts. In: Fenkart, Gabriele/Lembens, Anja/Erlacher-Zeitlinger, Edith (Hrsg.): Sprache, Mathematik und Naturwissenschaften. Innsbruck/Wien/ Bozen: Studien Verlag, S. 26-46.

Bullock, Alan (1975): A language for life - Report of the Committee of Enquiry appointed by the Secretary of State for Education and Science under the Chairmanship of Sir Alan Bullock F.B.A. London: Her Majesty's Stationery Office (HMSO). http://www.educationengland.org.uk/documents/bullock/ bullock1975.htm . Abgerufen am 16.03.2016.

Carbone, John N./Ekwaro-Osire, Stephen (2010): A Knowledge Component Framework for Enhancing Transdisciplinary Knowledge Assimilation. In: Transdisciplinary Journal of Engineering \& Science. Vol. 1, pp. 84-104.

Defila, Rico/Di Giulio, Antonietta (1999): Evaluationskriterien für inter- und transdisziplinäre Forschung. In: Panorama. Sondernummer 99, S. 5-11.

Feilke, Helmut (2012): Bildungssprachliche Kompetenzen. In: Praxis Deutsch. Jg. 39. H. 233, S. 4-13.

Kernen, Nora/Riss, Maria (2012): Textschwierigkeiten in Lehrmitteln für den naturwissenschaftlichen Unterricht in der Sekundarstufe I - Eine Analyse von der 
Pädagogischen Hochschule FHNW Zentrum Lesen. Beratung: Th. Lindauer, C. Schmellentin. http://www.zentrumlesen.ch. Abgerufen am 16.03.2016.

Klein Thompson, Julie (2010): A taxonomy of interdisciplinarity. In: Frodeman, Robert/Klein Thompson, Julie/Mitcham, Carl (Eds.): The Oxford Handbook of Interdisciplinarity. Oxford: Oxford University Press, pp. 15-30.

Leutner, Detlev/Klieme, Eckhardt/Meyer, Katja/Wirth, Joachim (2004): Problemlösen. In: PISA-Konsortium Deutschland (Hrsg.): Pisa 2003. Der Bildungsstand der Jugendlichen in Deutschland - Ergebnisse des zweiten internationalen Vergleichs. Münster/New York/München/Berlin: Waxmann, S. 147-175.

Lindauer, Thomas/Riss, Maria/Schmellentin, Claudia (2012): Empfehlungen für die sprachbewusste Gestaltung von Lehrmitteln. http://www.zentrumlesen.ch. Abgerufen am 16.03.2016.

Lindauer, Thomas/Schmellentin, Claudia/Beerenwinkel, Anne/Hefti, Claudia/Furger, Julienne (2013): Fachlernen und Sprache: Sprachbewusst unterrichten - Eine Unterrichtshilfe für den Fachunterricht. Bildungsraum Nordwestschweiz. http:// www.fhnw.ch/ppt/content/pub/fachlernen-und-sprache-sprachbewusst-unterrichten-eine-unterrichtshilfe-fur-den-fachunterricht. Abgerufen am 16.03.2016.

Lindauer, Thomas/Senn Werner (Hrsg.) (2008-2016): Die Sprachstarken 2-9. Baar: Klett \& Balmer.

Morek, Miriam/Heller, Vivien (2012): Bildungssprache - Kommunikative, epistemische, soziale und interaktive Aspekte ihres Gebrauchs. In: Zeitschrift für angewandte Linguistik Jg. 57. H. 1, S. 67-102.

Nagy, William/Townsend, Dianna (2012): Words as Tools: Learning Academic Vocabulary as Language Acquisition. Reading Research Quarterly. Vol. 47. No. 1, pp. 91-108.

Rincke, Karsten (2010): Alltagssprache, Fachsprache und ihre besonderen Bedeutungen für das Lernen. In: Zeitschrift für Didaktik der Naturwissenschaften. H. 16, S. 235-260.

Schleppegrell, Mary J. (2004): The language of schooling. A Functional Linguistic perspective. Mahwah, N.J.: Lawrence Erlbaum Associates.

Schmellentin, Claudia/Schneider, Hansjakob/ Hefti, Claudia (2011): Deutsch (als Zweitsprache) im Fachunterricht - am Beispiel Lesen. In: Leseforum. ch. Jg. 2. H. 3. http://www.leseforum.ch/sysModules/obxLeseforum/Artikel/ 460/2011_3_Schmellentin_Schneider.pdf. Abgerufen am 16.03.2016.

Schmellentin, Claudia/Dittmar, Miriam/Gilg, Eliane/Schneider, Hansjakob (im Druck): Sprachliche Anforderungen in Biologielehrmitteln. In: Ahrenholz, Bernt/Hövelbrinks, Britta/Schmellentin, Claudia (Hrsg.): Fachunterricht und Sprache in schulischen Lehr-/Lernprozessen. Tübingen: Narr. 
Schrader, Viola (2013): Geschichte als narrative Konstruktion - Eine funktional-linguistische Analyse von Darstellungstexten in Geschichtsschulbüchern. Münster: LIT.

Shanahan, Cynthia/Shanahan, Timothy/Misschia, Cynthia (2011): Analysis of expert readers in three disciplines: History, mathematics, and chemistry. In: Journal of Literacy Research. Vol. 43. No. 4, pp. 393-429.

Stock, Paul/Burton, Rob J. F. (2011): Defining Terms for Integrated (Multi-InterTrans-Disciplinary) Sustainability Research. In: Sustainability. Vol. 8. No. 3, pp. 1090-1113.

von Borries, Bodo (2011): Schulbuch-Gestaltung und Schulbuch-Benutzung im Fach Geschichte. Zwischen empirischen Befunden und normativen Überlegungen. In: Handro, Saskia/Schönemann, Bernd (Hrsg.): Geschichtsdidaktische Schulbuchforschung. 2. Aufl. Münster: LIT, S. 39-51.

Wittmann, Erich Ch. (1995): Mathematics Education as a «Design Science〉. In: Educational Studies in Mathematics. Vol. 29. No. 4, pp. 355-374. 